\title{
t)
}

INDUSTRIALES

ETSII | UPM

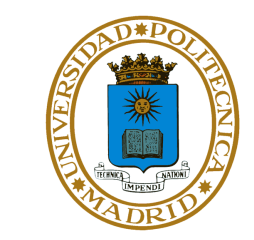

POLITÉCNICA

Universidad Politécnica de Madrid

Escuela Técnica Superior de Ingenieros INDUSTRIALES

\section{RELEVANT FRAMEWORK FOR SOCIAL APPLICATIONS OF INTERNET OF THINGS BY MEANS OF MACHINE LEARNING TECHNIQUES}

\author{
TESIS DOCTORAL \\ DoCTORADO INTERUNIVERSITARIO EN ECONOMÍA Y \\ Gestión DE LA InNOVACión (DEGIN)
}

XIAOCHEN ZHENG, MScEng

Supervisor:

Prof. JOAQUÍN ORDIERES MERÉ 

Tribunal nombrado por el Magfco. y Excmo. Sr. Rector de la Universidad

Politécnica de Madrid, el día de de 2019.

Presidente:

Vocal:

Vocal:

Vocal:

Secretario:

Suplente:

Suplente:

Realizado el acto de defensa y lectura de la Tesis el día de de 2019 en la Escuela Técnica Superior de Ingenieros Industriales.

Calificación:

El PRESIDENTE LOS VOCALES

\section{EL SECRETARIO}





\section{Doctorate in Economics and Innovation Management}

The Interuniversity Master and PhD Programs in Economics and Innovation Management are implemented jointly by Universidad Autónoma de Madrid (UAM), Universidad Complutense de Madrid (UCM) and Universidad Politécnica de Madrid (UPM), enabling students to access the resources of the three universities, regardless of the center where they are enrolled. These programs offer teaching, professional competence, and initiation to advanced research in an area of rising influence in contact with leading European research teams. The main objectives of DEGIN are as follows:

- To train individuals who are able to address and solve problems of scientific and technological nature in the field of economics and innovation management through independent and original research work to expand the knowledge frontier further.

- To train researchers capable of disseminating research results through scientific publications with a recognized review process and impact index.

- To offer society a group of individuals who are able to elaborate, assess and critique new scientific and technological development research proposals in the field of economics and innovation management.

- To train researchers eligible for jobs requiring a PhD Degree as postdoctoral researcher, university professor, researcher at institutes or businesses and other jobs requiring creativity, leadership and management in the area of technology.

- To contribute to raise awareness of the scientific activity as a career and of its ethical implications for society. 



\section{Contents}

\begin{tabular}{|l|l|l|l}
\hline Acknowledgment & I
\end{tabular}

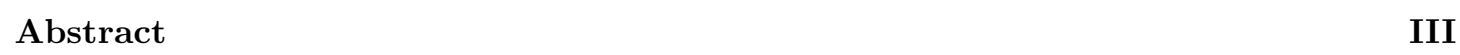

Resumen V V

$\begin{array}{lll}1 & \text { Introduction } & 1\end{array}$

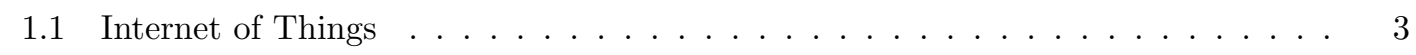

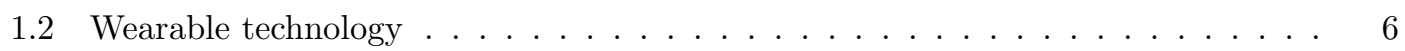

1.2 .1 The application of wearable technology in healthcare . . . . . . . . . . 9

1.2 .2 Pebble smartwatch . . . . . . . . . . . . . . . . . . . 10

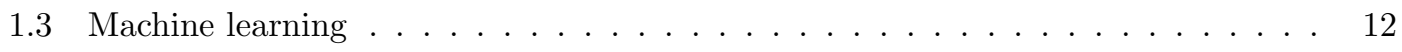

1.3 .1 Main algorithms $\ldots \ldots \ldots \ldots \ldots$

1.4 Essential Tremor . . . . . . . . . . . . . . . . . . . . . . . . . . . . . . . 17

\begin{tabular}{llr}
\hline 2 & Methodology & 19
\end{tabular}

$2.1 \quad$ Framework $\ldots \ldots \ldots \ldots$

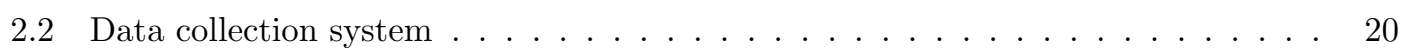

2.3 Experiments . . . . . . . . . . . . . . . . . . . . . . . . . . . . 21

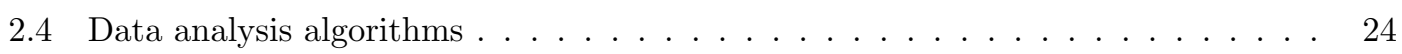

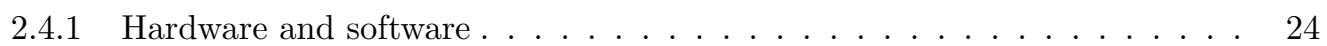

2.4 .2 Tremor identification . . . . . . . . . . . . . . . . . . 24

2.4 .3 Activity recognition . . . . . . . . . . . . . . . . . 24

2.4 .4 Activity-aware tremor evaluation $\ldots \ldots \ldots \ldots \ldots$

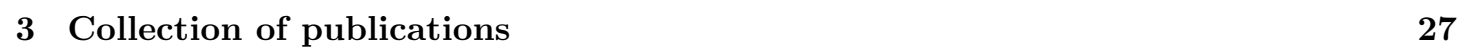

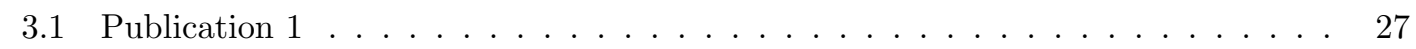

3.2 Publication $2 \ldots \ldots \ldots \ldots$

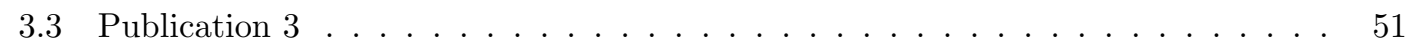

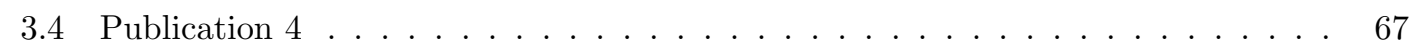

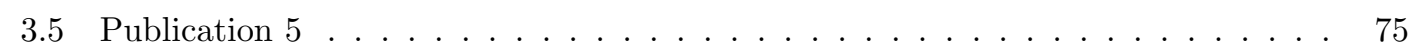


\begin{tabular}{|lr}
\hline 4 Discussion & 97
\end{tabular}

4.1 Contributions of each publication . . . . . . . . . . . . . . . . . . . 97

4.2 Limitations . . . . . . . . . . . . . . . . . . . . 100

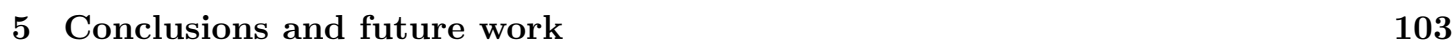

5.1 Conclusions . . . . . . . . . . . . . . . . . . . . . 103

5.2 Future work . . . . . . . . . . . . . . . . . . . . 105

\begin{tabular}{lr}
\hline Bibliography & 115
\end{tabular}

\begin{tabular}{|rr}
\hline Appendices & 117
\end{tabular}

\begin{tabular}{|rr}
\hline A ESPCO2014 conference paper & 119
\end{tabular}

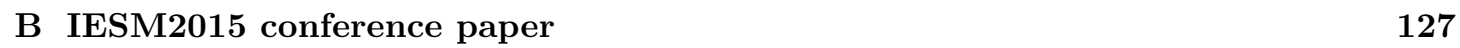

\begin{tabular}{|lr}
\hline C Healthcom2018 conference paper & 137 \\
\hline
\end{tabular} 


\section{Acknowledgment}

I would like to express my sincere gratitude to my advisor Professor Joaquín Ordieres-Meré for the support during my doctoral study and research. This thesis could not be possible without his guidance. His enthusiasm, diligence, patience and immense knowledge showed me the best example of a good professor and researcher and it will encourage me in my future career.

I would like to thank all my fellow doctoral students and colleagues of the university for their friendship, accompany, cooperation and encouragement, with a special mention to Dr. Bing Gong, Dr. Fadi Alshrouf, Dr. Ilaria de Sanctis, José Franco, Shengjing Sun, Ermal Hetemi, Isaac Lemus, Ebru Susur, Hossein Zarei, Jakob and Frano. There could not have been so much fun in my life as a doctoral student without all of you.

I am grateful to all the neurologists, Alba Vieira Campos, Yolanda Aladro and Sergio Labrador Marcos, and all the patients during the ET project. It was a great pleasure to cooperate with you.

I am also grateful to Prof. Ravi Vatrapu and Prof. Raghava Mukkamala from the Centre for Business Data Analytics for hosting me during my visit to Copenhagen Business School.

In addition, I would like to express my gratitude to my co-authors beside the ones mentioned above, Prof. Carlos Rodríguez-Monroy, Meiqing Wang and Qing Guo, for making all those publications possible.

A special gratitude goes to China Scholarship Council for offering me the CSC scholarship making it possible for me to start my doctoral study at the very beginning.

Finally, last but by no means least, I would like to thank all my family members, especially my parents and my brother, for supporting me throughout my life. You have always been the most important motivation for me to continue and finish the doctoral study. 


\section{Abstract}

The recent adaptation of wireless technologies and the widespread deployment of spatially distributed devices with embedded identification, sensing and actuation capabilities created the Internet of Things (IoT). This promising paradigm has been developing explosively in recent years. It is believed to be the next revolutionary technology by bringing the traditional sense of Internet into the physical realm. Billions of inter-connected smart things are streaming out a huge amount of data every moment and promote the world into "big data" era. Unimaginable potential value can be mined from these data supported by advanced data storage and analysis technologies, such as machine learning and cloud computing.

With the help of advanced data mining tools, IoT can bring great benefits for various domains of the society including healthcare. The healthcare industry has been dramatically changed because of the information technology revolution that started in the last century. New technologies such as telemedicine, digital hospital and e-health have been widely applied during the past decades and now the rapidly development of IoT and machine learning is promoting healthcare from digital into intelligent.

As an important aspect of IoT, wearable technology has also shown a surge in the past decade. Different types of wearable devices containing various embedded sensors have been introduced into the market with affordable prices. Large amounts of health-related data are generated by these wearable devices related to different daily activities. These low-cost data, supported by mobile computing and machine learning techniques, make it possible to develop Smart Decision Support Systems (SDSS) which can be beneficial to the long-term activity monitoring, remote disease diagnosis, emergency medical alerts promoting and so on.

As one of the most important tools to realize Artificial Intelligence (AI), machine learning has been growing explosively in the past decades with the development of Internet. Various of machine learning techniques have been widely used to implement different data mining tasks, among which, deep learning has shown outstanding performance in recent years due to the availability of big data.

Our research aims to address the applications of IoT technology supported by advanced machine learning techniques in different social areas, especially in healthcare. A general application framework was constructed, which includes data collecting and transferring, data storage and analysis, and analysis result sharing. In order to verify the feasibility of proposed application 
framework, a practical human movement data collecting system was developed based on wearable technology. It includes three modules: a smart watch, a smartphone and a remote NoSQL database. The system was applied in a hospital to collect daily activity and tremor data from patients with Essential Tremor (ET). Advanced machine learning techniques, including deep learning, were adopted to realize Human Activity Recognition (HAR) and ET evaluation tasks. Through proper data preprocessing and data transformation, based on the collected acceleration data, the proposed models could recognize a series of human daily activities and classify tremor levels with high accuracy. These models could enable neurologists remotely and continuously monitor ET patients' daily activities and the corresponding tremor situation. The evaluation result could help them to improve the treatment plans. This case proved the feasibility of the presented IoT application framework and similar applications could be developed in other scenarios.

As one of the future research directions, a personal health data sharing system based on blockchain technology was proposed in the end of the study. The aim is to protect the privacy and security during the data sharing process. 


\section{RESUMEN}

Los desarrollos recientes de las tecnologías inalámbricas y el despliegue extendido de dispositivos distribuidos espacialmente con capacidades de identificación, detección y actuación integradas crearon la Internet de las cosas (IoT). Este paradigma prometedor se ha desarrollado de forma explosiva en los últimos años. Se cree que es la próxima tecnología revolucionaria al llevar el Internet tradicional al ámbito físico. Miles de millones de cosas inteligentes interconectadas están transmitiendo una gran cantidad de datos a cada momento y promocionando el mundo en la era del "big data". El valor potencial inimaginable se puede extraer de estos datos respaldados por tecnologías avanzadas como aprendizaje automático y la computación en la nube.

Con la ayuda de herramientas avanzadas de minería de datos, IoT puede aportar grandes beneficios para varios dominios de la sociedad, incluida la atención de salud. La industria de la salud ha cambiado drásticamente debido a la revolución de la tecnología de la información que comenzó en el siglo pasado. Las tecnologías nuevas, como la telemedicina, el hospital digital y la atención sanitaria electrónica, se han aplicado ampliamente durante las últimas décadas y ahora el rápido desarrollo de IoT y el aprendizaje automático promueven la atención de salud de digital a inteligente.

Como un aspecto importante de IoT, la tecnología portátil también ha mostrado un aumento rápido en la última década. Se han introducido en el mercado diferentes tipos de dispositivos portátiles que contienen varios sensores integrados con precios asequibles. Estos dispositivos portátiles generan grandes cantidades de datos relacionados con la salud durante diferentes actividades diarias. Estos datos de bajo costo, respaldados por técnicas de computación móvil y aprendizaje automático, hacen posible el desarrollo de sistemas de soporte de decisiones inteligentes (SDSS) que pueden ser beneficiosos para el monitoreo de actividades a largo plazo, el diagnóstico remoto de enfermedades y la promoción de alertas médicas de emergencia.

Como una de las herramientas más importantes para realizar la Inteligencia Artificial (IA), el aprendizaje automático ha crecido de manera explosiva en las últimas décadas con el desarrollo de Internet. Varias de las técnicas de aprendizaje automático se han utilizado ampliamente para implementar diferentes tareas de minería de datos, entre las cuales, el aprendizaje profundo ha mostrado un rendimiento sobresaliente en los últimos años debido a la disponibilidad de "big data".

Nuestra investigación tiene como objetivo abordar las aplicaciones de la tecnología IoT respal- 
dada por técnicas avanzadas de aprendizaje automático en diferentes áreas sociales, especialmente en la atención de salud. Se construyó un marco de aplicación general, que incluye la recopilación y transferencia de datos, el almacenamiento y el análisis de datos, y La entrega de resultados de análisis a los usuarios. Con el fin de verificar la viabilidad del marco de aplicación propuesto, se desarrolló un sistema práctico de recolección de datos de movimiento humano basado en tecnología portátil. Incluye tres módulos: un reloj inteligente, un teléfono inteligente y una NoSQL base de datos remota. El sistema se aplicó en un hospital para recopilar datos de actividad diaria y temblor de pacientes con Temblor Esencial (ET). Se adoptaron técnicas avanzadas de aprendizaje automático, incluido el aprendizaje profundo, para realizar tareas de Reconocimiento de Actividad Humana (HAR) y evaluación de ET. A través del procesamiento adecuado y la transformación de los datos, los modelos propuestos podrían reconocer una serie de actividades diarias humanas y clasificar los niveles de temblor con una alta precisión. Estos modelos podrían permitir a los neurólogos monitorear de forma remota y continua las actividades diarias de los pacientes con ET y la correspondiente situación de temblor. El resultado de la evaluación podría ayudarles a mejorar los planes de tratamiento. Este caso demostró la viabilidad del marco de aplicación IoT presentado y otras aplicaciones similares podrían desarrollarse en otros escenarios.

Como una de las futuras direcciones de investigación, al final del estudio se propuso un sistema de intercambio de datos personales basado en la tecnología blockchain. El objetivo es proteger la privacidad y la seguridad durante el proceso de intercambio de datos. 


\section{Chapter 1}

\section{Introduction}

Since five thousand years ago, when the Sumerians invented the Cuneiform script, the way of recording and sharing knowledge has been upgrading all along the human history. In ancient China, the phrase xue fu wu che was used to describe a scholar's wealthy in knowledge, meaning the knowledge concealed his mind is more than that been contained in five cartloads of "books". Those contents may be less than that contained in this thesis, because that happened in more than two thousand years ago, when the "books" were manually written on bamboo slips. Centuries later, paper and printing techniques were invented. Then the real book appeared. During the past millennium, these inventions had greatly increased the development of human civilization. At the same time, the way of exchanging information has also been upgrading all along the history. After the Battle of Marathon, the Greek soldier Philippides run all the way back to Athens to report the victory. Different forms of postal systems have been serving the exchange of information in written form for thousands of ages. The appearing of electrical telegraph system in 19th century brought a revolution for information exchange and the invention of telephone system made it even faster and easier.

In the 1950s computer was invented and a decade later in the 1960s the first ARPANET link was established between the University of California Los Angeles and the Stanford Research Institute 12 Several other networks were built in different corners of the world at the same period, such as the NPL, Merit Network, CYCLADES, X.25 and public data networks, UUCP and Usenet. 3 By unifying and joining these separated networks, the Internet was born in the 1980s and then grew up explosively. The information technology revolution happened which totally reformed our way of producing, storing and sharing information.

In 2010, Google CEO Eric Schmidt famously said, "There were five Exabytes of information created between the dawn of civilization and 2003, but that much information is now created in two days." In 2015, the pace increased to every 12 hours to create and copy that amount of data 4. This is absolutely not the end of the story; instead, it may be just the beginning of another

1 https://computer.howstuffworks.com/arpanet $1 . \mathrm{htm}$

2 http://www.netvalley.com/cgi-bin/intval/net_history.pl?chapter=1

3 https://en.wikipedia.org/wiki/History_of_the_Internet\#cite_note-16

${ }^{4}$ http://www.langrandhealth.com/its-time-for-info/ 
revolution. The growing speed of Internet and the data produced by it show no signs of slowing down. What will be the next step of information technology evolution? Internet of Things (IoT) may be one the most possible answers 5

Since first proposed in 1999 by Kevin Ashton [7, the term IoT has been attracting more and more attention from both academic and industrial fields. It appears in research articles, technology newspapers, electrical appliance advertisements, and so on. Many researchers believe it is resulting in another technological revolution after the Internet [42]. No one knows what will happen in the future, but there is no doubt about the great potential of this new technology or, more precisely, a series of technologies. It is bring the Internet into the real world by connecting billions of devices into a whole network. These devices are streaming out a huge amount of data in every moment and leading us to the Big data era. The Big data not only means the "big" amount of data, but also refers to the unimaginable potential value that can be mined from these data. To transfer this potential into real benefits, a series of supporting technologies like cloud computing and machine learning are essential.

These above-mentioned topics have been attracting more and more attention in recent years. The statistic of Google search trends from 2004 to 2017, as shown in Figure 1.1, shows that in 2008 cloud computing started to gain dramatically increasing popularity and later in $2012 \mathrm{big}$ data started to become popular and the same happened to IoT and machine learning. Similar trends can be found in academic area. The amount of publications related to these topics increased dramatically after 2010 .

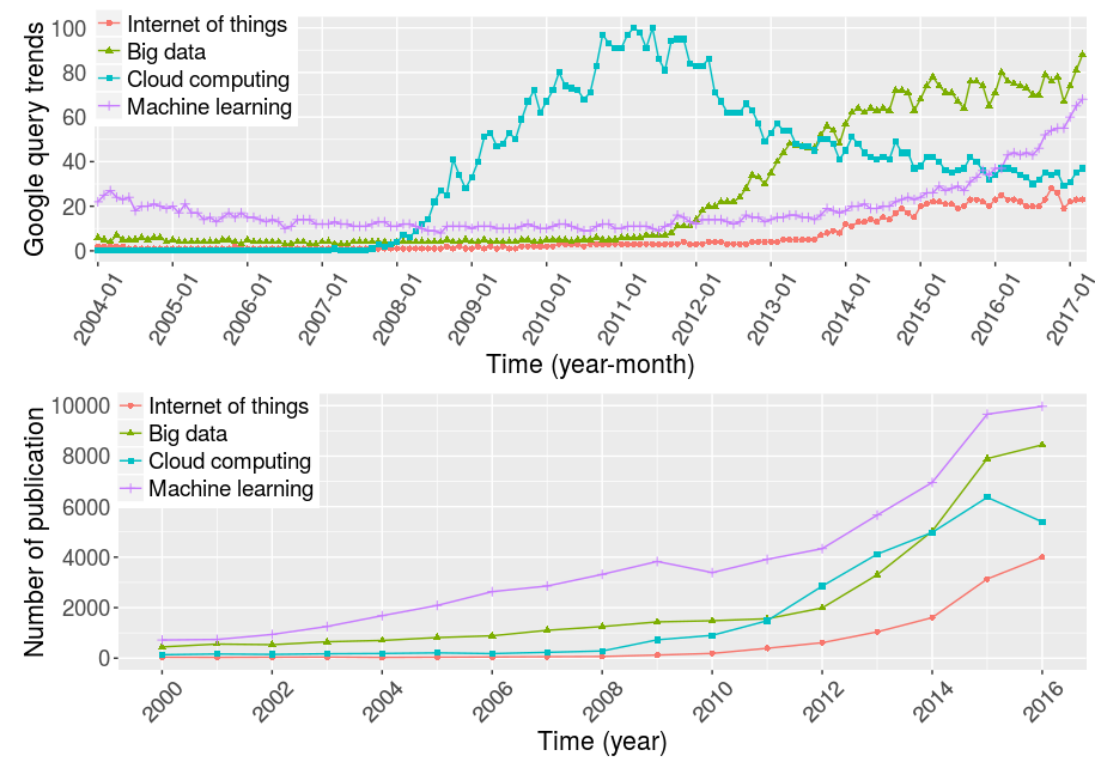

Figure 1.1: Google search statistics and number of publications about IoT, big data, cloud computing and machine learning

Our research focused on the applications of IoT in different social areas integrated with a series of computing and data mining techniques. The aims of this study include:

- Design a framework to integrate IoT and machine learning technologies and apply them

5 http://www. langrandhealth.com/its-time-for-info/ 
in different domains.

- Develop a prototype system to realize the main functions required by the previously designed application framework, such as sensor data collection, big data storage, advanced data analysis and result feedback.

- Apply the prototype system to a practical case to verify its feasibility and explore proper approaches to IoT sensor data transferring, storage and sharing.

- Use advanced machine learning techniques to analyze the data collected by the prototype system and figure out how they can be used to solve practical problems.

The main enabling technologies of our research are briefly introduced in the following sections, including IoT, wearable technology, machine learning and deep learning. The introduction will mainly cover the development status, applications, challenges and future trends of each technology. The Essential Tremor (ET) disease, which was the main study case in our research is also introduced in the end of this chapter.

\section{$1.1 \quad$ Internet of Things}

The term "Internet of Things" (IoT) was first proposed in 1999 by Kevin Ashton, the co-founder and former executive director of the Auto-ID Center, as the title of a presentation at Procter \& Gamble $(\mathrm{P} \& \mathrm{G})$ in the context of supply chain management 7 . The meaning at that time was linking things or objects in supply chain to the Internet using the new idea of Radio Frequency IDentification (RFID). With the fast growth of new technologies in the past decades, its meaning has been more inclusive, covering a wide range of applications like transportation, healthcare, smart environment (home, office, plants), retail, supply chain management, manufacturing, product lifecycle management, security and privacy and environment monitoring [101].

Many researchers and groups have proposed different definitions to IoT. According to a report of International Telecommunication Union [51, IoT is a series of technologies by embedding short-range mobile transceivers into a wide array of additional gadgets and everyday items, enabling new forms of communication between people and things, and between things themselves, to make anyone has the connectivity for anything from anytime and any place. Atzori et al. 8 suggested that IoT is syntactically composed of two terms. The first one pushes towards a network oriented vision of IoT, while the second one moves the focus on generic "objects" to be integrated into a common framework. So IoT can be realized in three paradigms: Internetoriented (middle-ware), things-oriented (sensors) and semantic-oriented (knowledge). In this definition, the main concepts, technologies and standards are highlighted and classified with reference to the IoT visions that contribute to characterize best. Jayavardhana Gubbi et al. 42 . proposed their definition of IoT on the basis of Atzori [8, Cluster of European research projects on the IoT 101] and Bélissent's 17] opinions: "interconnection of sensing and actuating devices 
providing the ability to share information across platforms through a unified framework, developing a common operating picture for enabling innovative applications". It is achieved by seamless ubiquitous sensing, data analytics and information representation with Cloud computing as the unifying framework.

Things with the ability of interconnecting and interacting are considered as the heart of the IoT. Things can be varying degrees of smart. The characteristics of a thing includes: identity, interactivity, shadowing, and autonomy [55], which means:

- Identity Things need to be identifiable either by type or by instance. For example, RFID tags can store Electronic Product Codes (EPC), IP addresses or hard-coded numbers can also be used to identify things.

- Interactivity Things have to be connected to a network or monitoring device all the time or occasionally, which divides things into passive things, such as RFID tags only need to report their presence from time to time, and active things, which can be connected all the time or only when they need to exchange information.

- Shadowing This means a software program, or a virtual instance, is running somewhere that knows everything about the physical item and can act on its behalf. This is also termed a cyber-object or a virtual agent.

- Autonomy Things need to be treated and monitored individually, and they must operate independently of a remote control. They can exhibit various degrees of smartness, which means to have awareness or even a level of decision-making capability.

Inter and intra network communication ability is another essential component of IoT. A smart network should have characteristics like standardization and openness, data and object accessibility, and multi-functionality 84 . It should use open standards for application layers interfacing with the physical world (i.e. tags and sensors), for the communication between nodes and with the network; should have access to the collected data for users, stakeholders and developers; should be open and available for additional purposes.

Typically, the architecture of IoT includes three layers 62, 75]: perception layer, network layer, and application layer. The functions and components of each layer are described below:

- Perception Layer It interacts with physical devices and components through smart devices, such as RFID, sensors, actuators, and is usually implemented as the bottom layer in IoT architecture 9. Its main functions are to connect things into IoT network; to process the data produced by these things via deployed smart devices; and to transmit the processed information into upper layer via layer interfaces.

- Network Layer It is used to receive the processed information provided by perception layer and transmit it to the IoT hub, devices, and applications via integrated networks. It is usually implemented as the middle layer in IoT architecture. Various devices such as hubs, 
switches, gateways, cloud computing platforms, and various communication technologies, including Bluetooth, Wi-Fi, Long-Term Evolution, are integrated in this layer, making it the most important part of the whole IoT architecture 61 .

- Application Layer It is usually implemented as the top layer in IoT architecture and the main objective is to receives the data transmitted from network layer and to provide required services or operations, like storage service to backup received data into a database, or analysis service to evaluate the received data for predicting the future state of physical devices [4].

On the basis of the aforementioned three-layer architecture, the service-oriented architectures (SoAs) have recently been developed to establish a more flexible multilayer architecture for IoT, by adding a service layer between network layer and application layer 30,62 . SoA is a component-based model, which can be designed to connect different functional units or services of an application via interfaces and protocols 29, 38]. In the four-layer SoA-based IoT architecture, the service layer is located between network layer and application layer, providing services to support the application layer 8 . The service layer consists of service discovery, service composition, service management, and service interfaces 62 .

As a part of future trends and developments, potentialities offered by the IoT make possible the development of a huge number of applications, of which only a very small part is currently available to our society. Many are the domains and environments in which new applications would likely improve the quality of our lives [8. In a report of GigaOM Research [55], researchers listed some existed usage scenarios of IoT in different areas. For example, many passports, hotel room keys and ski passes now have RFID tags in them to help identify the owners; tracking devices can be injected into pets, and following the mad cow disease crisis in the UK, RFID has become a staple identification and tracking mechanism for livestock; smart roads and bridge monitoring sensors are also being used increasingly to dynamically map transport conditions; using a combination of sensors and predictive analytic, it is possible to identify, assess, and respond to potential faults before they occur; a remote monitoring system triggers events when an certain issue occurs, which can result in an ambulance being sent automatically without the need for a call; IoT can make processes more efficient by augmenting existing interactions and activities to improve productivity and reduce the costs of doing business.

In healthcare domain, which our research mainly focused on, many benefits could also be provided by the IoT technologies: 8,55

- Tracking It is the function of identifying a person or object in motion. This includes: realtime position tracking, such as patient-flow monitoring to improve workflow in hospitals; tracking of motion through choke points, such as access to designated areas; continuous inventory location tracking; and materials tracking to prevent left-ins during surgery, such as specimen and blood products.

- Identification and authentication It includes patient identification to reduce incidents 
harmful to patients, such as wrong drug/dose/time/procedure; infant identification in hospitals to prevent mismatching; medical staff authentication to grant access and to improve employee morale by addressing patient safety issues.

- Automatic data collection and transfer It is mostly aimed at reducing form processing time, process automation (including data entry and collection errors), automated care and procedure auditing, and medical inventory management.

- Integrated control As electronic devices, such as heart monitors, X-ray machines, and other equipment, are a mainstay of health care delivery, it is beneficial to connect them together. Control applications on computers, tablets or even smartphones can be developed to upload patient information automatically. This enables healthcare professionals to gain immediate access to all such information and help them make better decisions.

- Sensing Wearable sensors enable automatic diagnosing of patient conditions, providing real-time information on patient health indicators. Application domains include different telemedicine solutions, monitoring patient compliance with medication regiment prescriptions, and alerting for patient well-being. Heterogeneous wireless access-based remote patient monitoring systems can be deployed to reach the patient everywhere, with multiple wireless technologies integrated to support continuous bio-signal monitoring in presence of patient mobility. There will be more explanations in the next section about this application.

Fueled by the prevalence of devices enabled by open wireless technology such as Bluetooth, RFID, WiFi, and telephonic data services as well as embedded sensor and actuator nodes, IoT has stepped out of its infancy and is on the verge of transforming the current static Internet into a fully integrated future Internet 111]. According to the Global System for Mobile Communications Association (GSMA), the number of interconnected devices is expected to reach 24 billion by 2020 and this amounts to $\$ 1.3$ trillion revenue opportunities for mobile network operators alone spanning vertical segments such as health, automotive, utilities and consumer electronics.

\subsection{Wearable technology}

Much has changed since the invention of the first computer that was available for the masses. After several decades, computers have become smaller, more efficient, and more powerful. Modern computers no longer need to occupy huge spaces. They can even be pocketed and now worn as fully-functional gadget accessories [52], which has greatly pushed forward the development of wearable technology. Wearable technology or wearable computing is the study or practice of inventing, designing, building, or using miniature body-borne computational and sensory devices. Wearable devices may be worn under, over, or in clothing, or may also be themselves clothes [77]. And according to Tehrani [104], the terms "wearable technology", "wearable devices", and "wearables" all refer to electronic technologies or computers that are incorporated 
into items of clothing and accessories which can comfortably be worn on the body. These wearable devices can perform many of the same computing tasks as mobile phones and laptop computers; however, in some cases, wearable technology can outperform these hand-held devices entirely. Today's wearable devices are characteristically more powerful, more efficient, and more compact. They also possess a greater range of features and are more convenient to wear. Additionally, they feature better technologies in terms of displays, processors, batteries, and input and output systems. Wearable technology tends to be more sophisticated than handheld technology on the market today because it can provide sensory and scanning features not typically seen in mobile and laptop devices, such as biofeedback and tracking of physiological function 104 .

Generally, wearable technology will have some form of communications capability and will allow the wearer access to information in real time. Data-input capabilities are also a feature of such devices, as is local storage. Examples of wearable devices include watches, glasses, contact lenses, e-textiles and smart fabrics, headbands, beanies and caps, jewelry such as rings, bracelets, and hearing aid-like devices that are designed to look like earrings. While wearable technology tends to refer to items which can be put on and taken off with ease, there are more invasive versions of the concept as in the case of implanted devices such as micro-chips or even smart tattoos. Ultimately, whether a device is worn on or incorporated into the body, the purpose of wearable technology is to create constant, convenient, seamless, portable, and mostly hands-free access to electronics and computers.

The implications and uses of wearable technology are far reaching and can influence the fields of health and medicine, fitness, aging, disabilities, education, transportation, enterprise, finance, gaming and music. The goal of wearable technologies in each of these fields will be to smoothly incorporate functional, portable electronics and computers into our daily lives. Prior to their presence in the consumer market, wearable devices were primarily used in the field of military technology and had the biggest implications for healthcare and medicine 88].

Thanks to the advancement of various technologies, using wearable devices has now become less unwieldy and awkward. More efficient and smaller high capacity batteries provide enough power to run wearable devices; thinner and sharper displays now make it possible to create more comfortable means through which users can interact with their devices; touch input systems have become more responsive; sensors have also become smaller and processors have become faster without overheating or requiring active cooling systems. They are expected to develop faster and possibly result in another technological revolution in the years to come. Therefore, wearable technology is expected to be more useful in serving the scope of purposes listed below [52]:

- Enabling ubiquitous computing and wireless communications;

- Assisting visually-impaired and hard-of-hearing individuals in interacting with their environment;

- Aiding deaf-mutes in communicating with other people; 
- Recording and documenting activities, processes, and events (especially for scientists, or perhaps intelligence agents);

- Accessing and sharing information quickly and wirelessly;

- Making computations and preparing electronic documents on-the-go;

- Multimedia entertainment;

- Schedules setting and tracking;

- Capturing and sharing textual, audio, and visual data;

- Interacting with or controlling other electronic devices;

- Facilitating learning and instruction.

Wearable devices can be classified based on their function, appearance, proximity to the human body, and other parameters [21.78]. According to their functional properties and capabilities, the study 78 classified them into the following categories:

- Smartwatch Smartwatches are computerized devices or small computers intended to be worn on the wrist, and have expanded functionality that is often related to communication. They may be based on a mobile operating system or operate as smartphone-paired devices and provide an additional screen with which to show notifications. Other features include GPS navigation, fitness/health tracking, and activity analysis based on inertial sensors and so on.

- Smart Eyewear Smart glasses, smart contact lenses or smart goggles designed for Optical Head-Mounted Displays (OHMDs), Heads-Up Uisplays (HUDs), Virtual Reality (VR), Augmented Reality (AR), and Mixed Reality (MR). They can be divided into two categories: those paired with a smartphone, needed to see images on the smartphone screen, or separate ones, which require a wired connection with a source device.

- Fitness Tracker Fitness trackers, also known as activity trackers, are typically worn on the wrist, chest, or ears, and are designed to monitor and track outdoor sport activities and measure fitness-related metrics, such as the speed and distance of running, exhalation, pulse rate, and sleeping habits.

- Smart Clothing Smart clothing is usually used to monitor the physical condition of the wearer, including sportswear, consumer sports apparel (smart shirts and body suits), chest straps, medical apparel, work wear monitoring apparel, military apparel, and e-textiles 44]. It is typically in the form of shirts, socks, yoga pants, shoes, bow ties with secret cameras, helmets, and caps with a wide range of sensors and features. Wearable smart biometric devices have been applied in golf, soccer, athletics, racing, basketball, and baseball, to monitor the physical condition of players, to reduce the number of injuries and enhance 
team performance [18]. It has the potential to be exceedingly beneficial for firefighters, at construction sites, and for transportation $81,95,105]$.

- Wearable Camera These cameras are well-suited for creating first-person videos and photos in real time, including two types: small cameras that can be attached to either the body or clothes, or can even be worn in the ear; and larger cameras with mounting attachments to affix to caps or helmets. They have been applied in fall detection and monitoring ecological environments 32,87 .

- Wearable Medical Device They usually consist of one or more biosensors used to monitor a variety of physiological data to prevent disease, provide early diagnoses, and facilitate treatment and home rehabilitation 91. They are often grouped together with other wearables, such as activity monitors, smartwatches, smart clothing, and patches, and are all intended to help gather important data concerning the health of the patient using noninvasive sensors installed on the device.

Various types of sensors are used in wearable devices depending on the intended application. The study of 78 divided them into four major groups according to their features: (1) environmental sensors, such as light sensors, temperature sensors, sound sensors, humidity sensors, flame sensors, fume sensors, and barometric sensors; (2) biosensors, including body temperature sensors, heart-rate-monitoring sensors, electrocardiogram (ECG), electroencephalography (EEG), electromyography (EMG) sensors, blood pressure sensors, and glucose level sensors; (3)location tracking sensors, such as GPS, altimeter, magnetometer, compasses, and accelerometers; (4)other sensors, like camera sensors, communication sensor, ultrasonic sensors, infrared receiver (IR) sensors and so on.

\subsubsection{The application of wearable technology in healthcare}

In recent years, wearable technology has been applied in many domains and among which healthcare is one of the most important ones. Supported by artificial intelligence and big data, wearable devices are providing an added value to healthcare with a focus on activity monitoring, remote disease diagnosis, chronic disease therapy and so on. The application of wearable technology in healthcare offer multiple advantages to both professionals and the patients, such as earlier diagnosis based on precise medical parameters, personalization based on the needs of the patients, remote and real-time patient monitoring, better adherence to medication, more complete and precise information registry, optimum clinical decision, and lower healthcare cost 6 .

There are already many solutions available in the market and some of the recent examples are introduced below:

- Emma Watch Emma Watch 7 is a smartwatch designed for PD patients, as shown in

Figure 1.2a It analyzes the wearer's symptoms and then vibrates using a personalized

\footnotetext{
${ }^{6}$ https://www.wearable-technologies.com/2018/10/the-state-of-wearable-technology-in-healthcare-currentand-future/

${ }^{7}$ https://news.microsoft.com/en-gb/features/how-a-watch-helped-emma-write-again/
} 


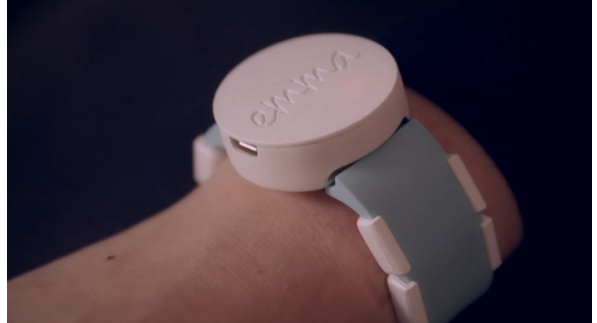

(a) Emma Watch

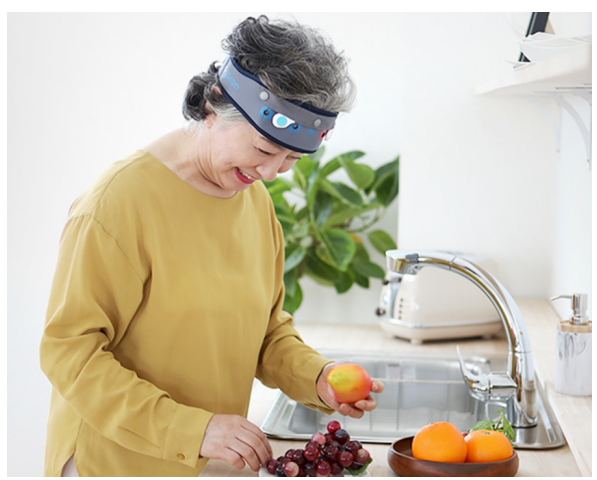

(c) MINDD STIMM wearable headband

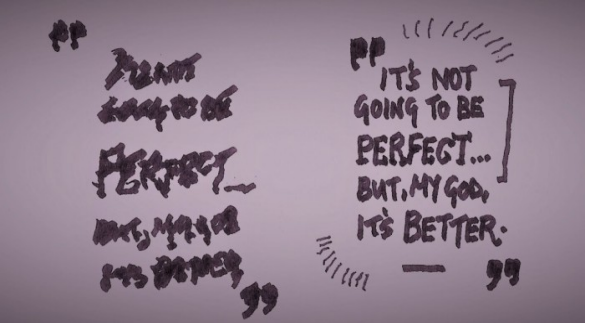

(b) Writing before and after wearing Emma Watch

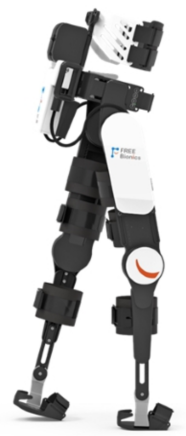

(d) FREE Walk exoskeleton

Figure 1.2: Application examples of wearable technology in healthcare

rhythm that's created using AI and the built-in sensors. These vibrations trick the brain into focusing on the wrist, reducing the signals that are sent to muscles and generating the tremors. It can significantly reduce patients' tremor during certain activities, as shown in Figure $1.2 \mathrm{~b}$.

- MINDD STIMM headband MINDD STIMM 8 wearable headband, as shown in Figure $1.2 \mathrm{c}$ is a wireless, non-invasive neuromodulation system for depression treatment, which is the most common mental condition worldwide and the leading cause of disability. It is a medical-grade system that uses weak current to stimulate human brain.

- FREE Walk exoskeleton FREE Walk 9 , as shown in Figure $1.2 \mathrm{~d}$ is an exoskeleton developed by FreeBionics to help people with limited mobility, muscle weakness or paraplegia to stand up and walk independently. Users can easily keep their balance and transfer their center of mass forward using this assistive exoskeleton technology that mimics the most natural human gait. It has a user-oriented design and adjustments for every new user can be done within five minutes.

\subsubsection{Pebble smartwatch}

As one of the most successful and popular wearable devices, smartwatches have been widely used in many areas related to healthcare, covering elderly/health monitoring, food and diet monitoring, Parkinson's disease and related movement disorders, medication adherence moni-

\footnotetext{
${ }^{8}$ http://www.ybrain.com/m21.php

${ }^{9}$ http://www.freebionics.com.tw/en
} 
toring, Alzheimer's disease, epilepsy/seizure patients, stroke patients, people with deaf or visual impairments 73. In our research a smartwatch named Pebble was used for human activity monitoring and tremor evaluation of patients with ET.

The Pebble smartwatch was designed based on a concept by Eric Migicovsky describing a watch that could display messages from a smartphone and select android devices [5]. Funded by the crowd funding platform Kickstarter, they released it in January of 2013 [53. Figure $1.3 \|^{0}$ shows the model used in our research. The features of Pebble are listed below:

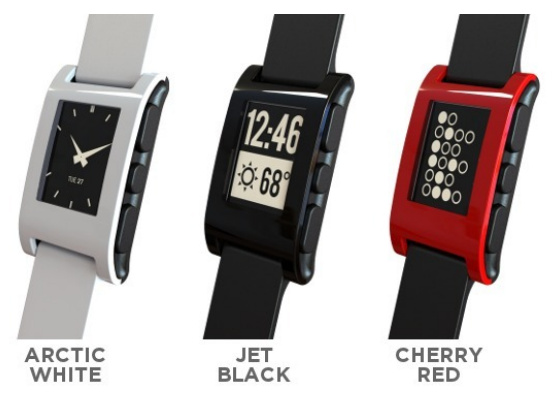

Figure 1.3: Pebble watches

- Hardware The Pebble features a ARM Cortex-M3 80 MHz processor, a black and white epaper display, a vibrating motor, a magnetometer, ambient light sensors, a 3D-accelerometer and a Bluetooth 4.0 receiver, enabling its use as an activity tracker [31. Pebble has been waterproofed to withstand five atmospheres of pressure, allowing one to shower, dive or swim while wearing the watch and its battery could last seven days 36. The Pebble hardware accelerometer can detect actions as light as taps, perform measures at a given frequency, and transmit samples in batches to save CPU time and processing 11.

- Software The Pebble smart watch has its own operating system, which is built on a FreeRTOS core, with added features on top. Alongside the operating system for the watch, there are also the core smartphone apps, which support Android 2.3.3 and iOS 5 and their later versions. As to the applications, until February 2014, the Pebble application store had over 1000 applications ${ }^{12}$ These include notifications for emails, calls, text messages, social media activity, stock prices; activity tracking (movement, sleep, estimates of calories burned); remote control of smartphones, cameras and home appliances; turn-by-turn directions using the GPS receiver in the smartphone.

- Pebble Software Development Kit (SDK) Pebble Technology released open Pebble Software Development Kit (SDK) released on April 12, 2013. The released SDK was limited to development for watch faces, simple applications and games. The second release of the SDK (now known as PebbleKit) was released on May 17, 2013, adding support for

\footnotetext{
${ }^{10}$ https://getpebble.com/discover

${ }^{11}$ https://developer.getpebble.com/2/guides/accelerometer.html

12 https://getpebble.com/discover
} 
two-way communication between Pebbles and smartphones running iOS or Android via the AppMessage framework. The latest Pebble SDK includes access to the accelerometer, as well as a Javascript API 13 .

The Pebble smartwatch was used in our research to collect motion data generated by the accelerometer sensor. A watch application was developed for this purpose using the SDK provided by Pebble. The collected data were used to analyze the daily activities and evaluate the tremor levels of the patients with ET. More details about the data collection system will be introduced in the methodology section.

\subsection{Machine learning}

In 1959, Arthur Samuel defined machine learning as "a field of study that gives computers the ability to learn without being explicitly programmed" 99 . According to Michie 83 machine learning is generally taken to encompass automatic computing procedures based on logical or binary operations that learn a task from a series of examples. Mitchell 85 provided a more formal definition: "a computer program is said to learn from experience $E$ with respect to some class of tasks $T$ and performance measure $P$, if its performance at tasks in $T$, as measured by $P$, improves with experience $E$ ". This definition is notable for its defining machine learning in fundamentally operational rather than cognitive terms. A more detailed definition is proposed by Taiwo [10], according to whom, machine learning is a process of building computer systems that automatically improve with experience, and implement a learning process. It learns the theory automatically from the data, through a process of inference, model fitting, or learning from examples. Take the spam E-mail filtering system as an example: a machine learning model could be trained on email messages and learn to distinguish spam from non-spam messages. After learning, it can then be used to classify new E-mail messages into spam and non-spam classes.

A major focus of machine learning research is to automatically produce models. A model could be a pattern, plan, representation, or description designed to show the main working of a system. Based on the desired outcome of the algorithm or the type of input available during the training process, machine learning algorithms can be classified into: supervised learning, unsupervised learning, semi-supervised learning, reinforcement learning, transduction and learning to learn 11 .

- Supervised learning algorithms are trained on labeled examples, i.e., input where the desired output is known. The supervised learning algorithm attempts to generalize a function or mapping from inputs to outputs which can then be used speculatively to generate an output for previously unseen inputs.

- Unsupervised learning algorithms operate on unlabeled examples, i.e., input where the

\footnotetext{
${ }^{13}$ https://developer.getpebble.com/2/
} 
desired output is unknown. Here the objective is to discover structure in the data (e.g. through a cluster analysis), not to generalize a mapping from inputs to outputs.

- Semi-supervised learning combines both labeled and unlabeled examples to generate an appropriate function or classifier.

- Transduction or transductive inference, tries to predict new outputs on specific and fixed (test) cases from observed, specific (training) cases.

- Reinforcement learning is concerned with how intelligent agents ought to act in an environment to maximize some notion of reward. The agent executes actions which cause the observable state of the environment to change. Through a sequence of actions, the agent attempts to gather knowledge about how the environment responds to its actions, and attempts to synthesis a sequence of actions that maximizes a cumulative reward.

- Learning to learn learns its own inductive bias based on previous experience.

- Developmental learning elaborated for robot learning, generates its own sequences (also called curriculum) of learning situations to cumulatively acquire repertoires of novel skills through autonomous self-exploration and social interaction with human teachers, and using guidance mechanisms such as active learning, maturation, motor synergies, and imitation.

\subsubsection{Main algorithms}

Many machine learning approaches, focusing on different domains, have been developed. Each approach has its advantage in one aspect and disadvantage in another, and sometimes it is necessary to combine different ones to get an ideal result. In our research, the following approaches were used:

- K-means K-means is one of the most commonly used and efficient clustering methods for performing unsupervised learning tasks. It uses prototypes (centroids) to represent clusters by optimizing the squared error function $33,46,74$. It is used to automatically partition a data set into $K$ groups by selecting $K$ initial cluster centers and then iteratively refining them as follows 108: Firstly, each instance is assigned to its closest cluster center; Then each cluster center is updated to be the mean of its constituent instances. The algorithm converges when there is no further change in assignment of instances to clusters.

- Classification And Regression Trees (CART) CART are machine-learning methods for constructing prediction models from data. The models are obtained by recursively partitioning the data space and fitting a simple prediction model within each partition. As a result, the partitioning can be represented graphically as a decision tree. Classification trees are designed for dependent variables that take a finite number of unordered values, with prediction error measured in terms of misclassification cost. Regression trees are for dependent variables that take continuous or ordered discrete values, with prediction 
error typically measured by the squared difference between the observed and predicted values 63 .

- Random Forest Random forests is an ensemble learning method for classification (and regression) that operate by constructing a multitude of decision trees at training time and outputting the class that is the mode of the classes output by individual trees. According to Leo Breiman [19], a random forest is a classifier consisting of a collection of tree-structured classifiers $\left\{h\left(x, \theta_{k}\right), k=1, \ldots\right\}$ where the $\left\{\theta_{k}\right\}$ are independent identically distributed random vectors and each tree casts a unit vote for the most popular class at input $\mathrm{x}$.

- Bayesian Network Bayesian Network is a probabilistic graphical model that represents a set of random variables and their conditional dependencies via a Directed Acyclic Graph (DAG). Formally, Bayesian networks are DAGs whose nodes represent random variables in the Bayesian sense: they may be observable quantities, latent variables, unknown parameters or hypotheses. Edges represent conditional dependencies; nodes represent variables that are conditionally independent of each other. Each node is associated with a probability function that takes a particular set of values as input for the node's parent variables and gives the probability of the variable represented by the node 100 . For example, if the parents are $m$ boolean variables then the probability function could be represented by a table of $2^{m}$ entries, one entry for each of the $2^{m}$ possible combinations of its parents being true or false.

This method is important for several reasons. It is very easy to construct, not needing any complicated iterative parameter estimation schemes. This means it may be readily applied to huge data sets. It is easy to interpret, so users unskilled in classifier technology can understand why it is making the classification it makes. And finally, it often does surprisingly well: it may not be the best possible classifier in any particular application, but it can usually be relied on to be robust and to do quite well [110].

- Support Vector Machines(SVMs) SVMs are supervised learning models with associated learning algorithms that analyze data and recognize patterns, used for classification and regression analysis. Given a set of training examples, each marked as belonging to one of two categories, an SVM training algorithm builds a model that assigns new examples into one category or the other, making it a non-probabilistic binary linear classifier. An SVM model is a representation of the examples as points in space, mapped so that the examples of the separate categories are divided by a clear gap that is as wide as possible. New examples are then mapped into that same space and predicted to belong to a category based on which side of the gap they fall on. In addition to performing linear classification, SVMs can efficiently perform a non-linear classification using what is called the kernel trick, implicitly mapping their inputs into high-dimensional feature spaces [110]. In a two-class learning task, the aim of SVM is to find the best classification function to 
distinguish between members of the two classes in the training data. The metric for the concept of the "best" classification function can be realized geometrically. For a linearly separable dataset, a linear classification function corresponds to a separating hyperplane $f(x)$ that passes through the middle of the two classes, separating the two. Once this function is determined, new data instance $x_{n}$ can be classified by simply testing the sign of the function $f\left(x_{n}\right) ; x_{n}$ belongs to the positive class if $f\left(x_{n}\right)>0$. Because there are many such linear hyperplanes, what SVM additionally guarantee is that the best such function is found by maximizing the margin between the two classes. Intuitively, the margin is defined as the amount of space, or separation between the two classes as defined by the hyperplane. Geometrically, the margin corresponds to the shortest distance between the closest data points to a point on the hyperplane.

- Artificial neural networks (ANN) ANN have been developed as generalizations of mathematical models of biological nervous systems 82 . The basic processing elements of neural networks are called artificial neurons, or simply neurons or nodes. In a simplified mathematical model of the neuron, the effects of the synapses are represented by connection weights that modulate the effect of the associated input signals, and the nonlinear characteristic exhibited by neurons is represented by a transfer function. The neuron impulse is then computed as the weighted sum of the input signals, transformed by the transfer function. The learning capability of an artificial neuron is achieved by adjusting the weights in accordance to the chosen learning algorithm.

The basic architecture consists of three types of neuron layers: input, hidden, and output layers 2]. In feed-forward networks, the signal flow is from input to output units, strictly in a feed-forward direction. The data processing can extend over multiple (layers of) units, but no feedback connections are present. Recurrent networks contain feedback connections. Contrary to feed-forward networks, the dynamical properties of the network are important. In some cases, the activation values of the units undergo a relaxation process such that the network will evolve to a stable state in which these activations do not change anymore. In other applications, the changes of the activation values of the output neurons are significant, such that the dynamical behavior constitutes the output of the network.

- Deep learning Deep learning methods are representation-learning methods with multiple levels of representation, obtained by composing simple but non-linear modules that each transform the representation at one level (starting with the raw input) into a representation at a higher, slightly more abstract level [59]. Deep learning is making major advances in solving problems that have resisted the best attempts of the artificial intelligence community for many years. It has turned out to be very good at discovering intricate structures in high-dimensional data and is therefore applicable to many domains of science, business and government. Many studies have proved that the use of deep learning can improve performance in a range of applications, especially in visual object recognition [58, 
speech recognition 6, 49, Natural Language Processing (NLP) 27, 112] and Human Activity Recognition (HAR) 96, 97, 113.

Some of the most widely applied deep learning models are Convolutional networks, also known as Convolutional Neural Networks (CNNs). They are a specialized kind of neural network for processing data that has a known grid-like topology, including time-series data and image data 39,60 . CNNs have been tremendously successful in most of the applications mentioned above. As the name indicates, the network employs a mathematical operation called convolution, which is a specialized kind of linear operation. As described in the book Deep Learning by Ian Goodfellow et.al 39] "Convolutional networks are simply neural networks that use convolution in place of general matrix multiplication in at least one of their layers". Different from the ordinary neuron networks, CNNs are based on four key ideas: local connections, shared weights, pooling and the use of many layers [59]. The typical architecture of a CNN includes a series of stages. The first stages are composed of convolutional layers and pooling layers (sub-sampling layers). The aim of the convolutional layer is to detect local conjunctions of features from the previous layer and the pooling layer could merge semantically similar features into one. Units in a convolutional layer are organized in feature maps, within which each unit is connected to local patches in the feature maps of the previous layer through a set of weights. The result of this local weighted sum is then passed through a non-linearity such as a Rectified Linear Unit (ReLU). A typical pooling method is max-pooling, which computes the maximum of a local patch of units in one feature map (or in a few feature maps). After stacking two or more stages of convolution, non-linearity and pooling layers, more convolutional and fully-connected layers are included in a typical CNN.

Recurrent Neural Networks (RNNs) are another kind of neural networks widely used for processing sequential data. Different from CNNs, which are specialized for processing a grid of values such as an image, RNNs are specialized for processing a sequence of values such as speech and language 39,59 . A RNN handles the variable-length sequence by having a recurrent hidden state whose activation at each time is dependent on that of the previous time 25. It processes an input sequence one element at a time, maintaining in their hidden units a 'state vector' that implicitly contains information about the history of all the past elements of the sequence [59. Traditionally, training RNNs to capture long-term dependencies has been proved difficult because the backpropagated gradients tend to either grow or shrink at each time step, so over many time steps they typically vanish (most of the time) or explode (rarely, but with severe effects) 16. In order to reduce the negative impacts of this issue, two dominant approaches have been proposed in recent years. The first approach is to devise a better learning algorithm than a simple stochastic gradient descent $15,79,89]$. The other approach, which has been more popular, is to augment the network with an explicit memory, or more specifically, to design a more sophisticated activation function consisting of affine transformation followed by a simple 
element-wise nonlinearity by using gating units 25,59]. The first proposal in the later direction is the long short-term memory (LSTM) networks that use special hidden units, the natural behavior of which is to remember inputs for a long time [50. More recently, another type of recurrent unit, named gated recurrent unit (GRU), was proposed [23]. RNNs employing either of these recurrent units have achieved outperforming results in tasks that require capturing long-term dependencies such as speech recognition [41] and machine translation 12,102 .

In recent years, deep learning has become one of the most widely used machine learning approaches owing to the advances in software infrastructure. Software libraries such as TensorFlow 1], Theano [14, PyLearn2 [40], Torch [26], Caffe 54] and MXNet 22]. have facilitated the application of deep learning in research projects or commercial products.

\subsection{Essential Tremor}

Essential tremor (ET) is one of the most common neurological disorders. It affects approximately $1 \%$ of the general population and $5 \%$ of the population over 65 years of age 68. ET is complex and progressive, and may be a family of related disorders rather than a single disease entity or a monosymptomatic disorder of little consequence 65. The mechanisms underlying ET remain far from well understood and no specific pathophysiological correlate has been determined [13 67. Epidemiological studies have identified several risk factors for ET, including age 34 , 71, 94, ethnicity $43,69,70$ and family history of ET [103. Traditionally, genetic factors have been viewed as important in the aetiology of ET [48, while some studies also considered the importance of environmental factors 66 .

It is believed that there is no increased risk of mortality for patients with ET 65 94. However, it affects activities of daily living and may cause psychological problems for the patients especially when tremor symptoms occur in public [45, 47,64]. The tremor of ET most commonly occurs in the arms, but other regions of the body are involved, especially the head (i.e., neck) and voice, and occasionally the trunk, legs and feet, tongue, and other facial musculature 28, 65]. Characterized by postural and kinetic tremor, ET may affect activities like handwriting, eating, dressing and self-care. The frequency of ET is between $4 \mathrm{~Hz}$ and $12 \mathrm{~Hz}$, and is inversely related to age, with older patients generally exhibiting tremor frequencies that are at the lower end of this range $20,35,107$. The amplitude, however, can vary significantly intra-individually and may be reduced by medication and mental relaxation [57,98.

Currently there's no cure for ET, but treatments that may help relieve the symptoms. The treatment of ET primarily involves medications, therapies, and surgery.

- Medicines may be used to lessen functional disability or reduce embarrassment. Medications for ET include ${ }^{14}$ beta-blockers, such as propranolol, which limit adrenaline and prevent tremors from getting worse; blood pressure medications, such as flunarizine, which

\footnotetext{
${ }^{14}$ https://www.healthline.com/health/essential-tremor
} 
limit adrenaline; anticonvulsant medications, such as primidone, which work to reduce the excitability of nerve cells; mild tranquilizers, such as alprazolam, which are also a treatment option.

- Physical therapies could improve ET patients' coordination and muscle control. Botox injections can also be done in the hands to weaken the muscles and minimize or stop shaking.

- Surgery is performed when other treatments fail to provide relief in severe, disabling cases. Surgical options include Deep Brain Stimulation, Focused Ultrasound Thalamotomy, Radiosurgical (Gamma Knife) Thalamotomy.

Due to a lack of disease-specific biological markers, the diagnosis of ET often proves difficult. Currently, rating scales such as the Tremor Research Group Essential Tremor Rating Assessment Scale (TETRAS) 86, Washington Heights-Inwood Genetic Study of Essential Tremor (WHIGET) tremor rating scale (wTRS) [72, and Fahne-Tolosae-Marin tremor rating scale 37 are used to evaluate ET during a clinical examination. Each tremor rating scale (TRS) rates tremor on a subjective, qualitative 0-4 scale. While these rating scales have clinical utility for clinicians treating patients with ET and are often used as an outcome measure for clinical drug trials, there are many limitations [93]. They require the presence of a clinician for scoring. The results are subject to clinical judgment and show significant intra and inter explorer variability. Moreover, they only provide an instantaneous, subjective and qualitative assessment of tremor intensity during a clinical visit and do not allow extended continuous monitoring of tremor fluctuation patterns throughout the day or in home environments. These limitations impede an objective, repeatable, and comprehensive evaluation of tremor severity [47].

The development of wearable technology provides a new approach to the diagnosis of ET. The frequency of ET is higher than the normal voluntary movements of humans, consequently, it is possible to detect tremor on the basis of the frequency difference 80. The feasibility of classifying tremor with the acceleration data collected from patient's wrist has also been reported [3. Different tremor data collection and analysis systems based on acceleration sensors have been developed and applied in many studies $47,56,76,90,106$. However, most of these systems are designed in the laboratory environment and will cause inconvenience to the patient in case of long-term application. For example, the KinesiaTM (CleveMed) system has shown a good correlation with scale tremor scores, for all, rest, positional and kinetic tremor [93]. Although this system is portable, it still limits the movement of the limb and it is hard to monitor the patient remotely due to the distance limitation. The development of wearable technology, such as smart watch, smart band and smart glasses, provides a new method to collect motion data more conveniently. The study presented 109 verified the practicability of using a smart watch to analyze and diagnose tremor. 


\section{Chapter 2}

\section{Methodology}

This research addresses the impact of emerging IoT technology on the society in connection with machine learning techniques. Low cost data access supported by knowledge-extraction machine learning approaches can have strong impact into social factors such as healthcare, by making it possible the so-called Smart Decision Support System (SDSS). It is very valuable for establishing forecast techniques helping citizens about best practices, monitoring them at home, taking care of their specific patterns, not only by gathering information but also by exploiting identified applicable rules and then, applying individual oriented strategies into monitoring processes.

\subsection{Framework}

A classical framework for the application of IoT supported by machine learning in social areas was designed at the inatial stage to guide the following research, as presented in Figure 2.1. The framework includes four layers: data collection layer, data preprocessing and uploading layer, data storage and analysis layer, and practical applications layer. The data collection layer focuses on the design of data collection methods using IoT and wearable technologies, development of necessary applications running on smart devices. The data preprocessing and uploading layer is a management system for managing all kinds of smart devices. It is responsible for receiving sensor data from smart devices and send it to NoSQL databases located in remote servers after simple processing and compressing if necessary. Data storage and analysis layer locates on the remote server. The data collected from the first two layers are stored in the distributed databases. The data are then analyzed with certain machine learning techniques. The analysis results will be used to develop practical applications to serve the users for different purposes.

This framework has been used in a practical case about improving the monitoring and diagnosis of ET. As discussed in previous sections, IoT and wearable technology have many applications in healthcare sector, with the possibility of using the smartphones with sensor capabilities as a platform for monitoring of medical parameters and drug delivery. The advantage gained is in prevention and easy monitoring of diseases, remote diagnosis and providing prompt medi- 
cal attention in cases of accidents, especially for people with diabetes, cancer, coronary heart disease, stroke, chronic obstructive pulmonary disease, cognitive impairments, seizure disorders and Alzheimer's disease. The data collection system, experiments and data analysis methods are introduced in the following sections.

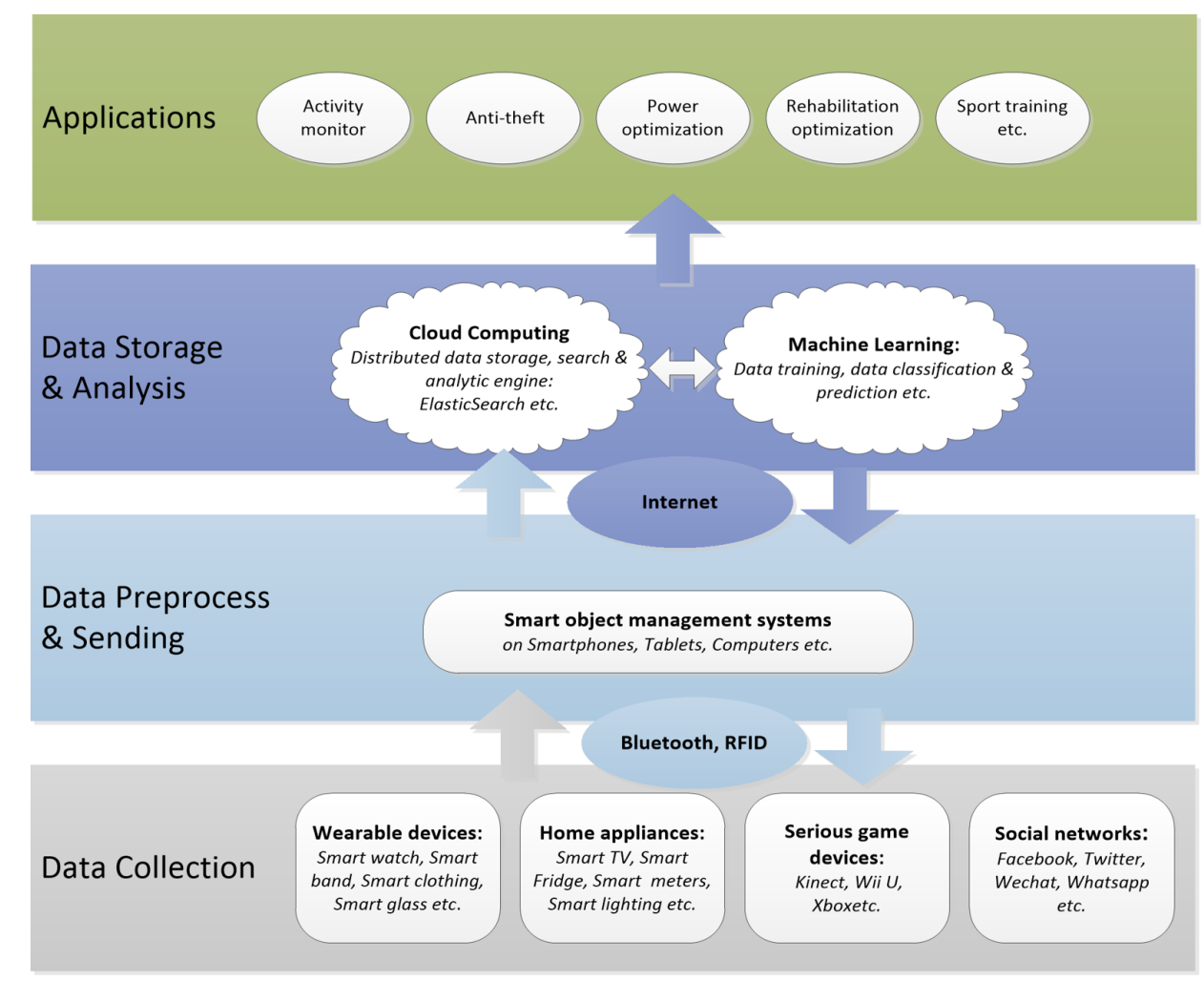

Figure 2.1: A classical framework for the application of IoT supported by machine learning in social areas

\subsection{Data collection system}

A human movement data collection system was developed at the first phase of the research. The system mainly contains three parts: (1) a Pebble smartwatch for recording the acceleration data related to user's arm movements; (2) an Android smartphone for receiving data from Pebble smartwatch and sends it to remote server together with data collected from its GPS sensor and accelerometer; (3) a data search and analytic engine on remote server for data storage and analysis. The structure of the system is shown in Figure 2.2 .

We developed an application for Pebble watch to collect the raw data from the accelerometer hardware and relay it to the smartphone. Several Pebble Application Program Interfaces (APIs) are used in our application, for instance, accelerometer, data logging, UI framework and event services. The application was developed on Cloud Pebble, which is a cloud development platform provided by Pebble company. Five values are recorded by the application: a timestamp value to identify the time when the action happens, acceleration values on the three axes and an angle value to show the angle between wearer's arm and the horizontal plane. 


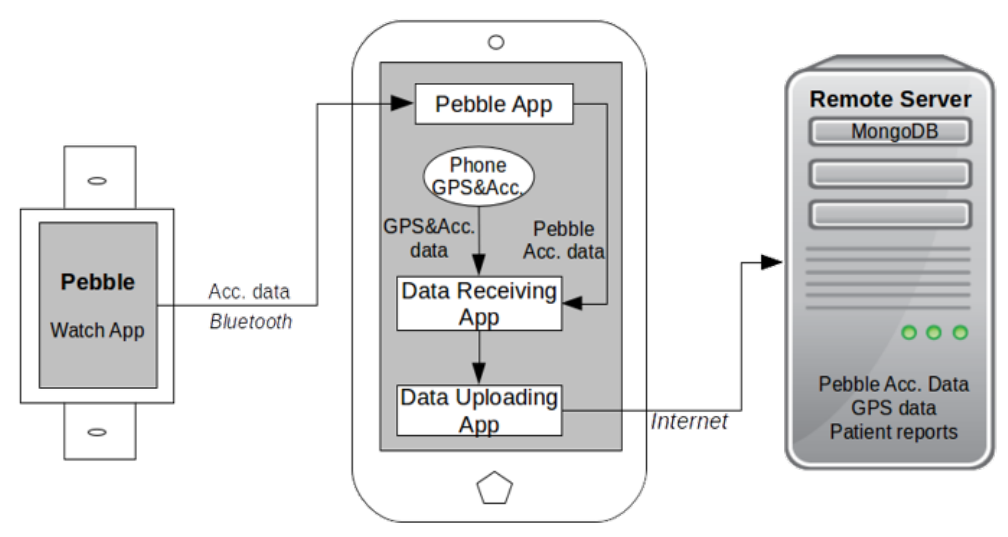

Figure 2.2: Three-layer architecture of human movement monitoring system

On the smartphone layer, a series of Apps which can receive data from the smartwatch and integrate them with the GPS data and other data collected from the sensors inside the smartphone. Besides, the users can also use these Apps to upload their self-evaluation results and personal reports as shown in Figure 2.3 .

All the data collected from the smartwatch and smartphone are integrated in the smartphone and then uploaded to remote server through Internet. On the remote server, a NoSQL database MongoDB 24 is used to manage the data received from the smartphone. It is a flexible and powerful open source, distributed, real-time search and analytic engine. NoSQL databases are developed to manage large volume and high complexity data produced due to the fast employment of smart devices. A NoSQL database is adopted in our system instead of a classical relational database because in our research we will not only deal with structural data like acceleration data, but also the non-structural data like patients' reports to help analyze their behaviors. Both the size and the type of data cannot be defined in advance which requires the database to be very flexible. Classical relational database cannot fill these requirements while the NoSQL database can solve these problems very well.

More details about the data collection systems are introduced in two conference papers [114, 115] and the full text are attached in the appendix of this thesis.

\subsection{Experiments}

The experiments related to ET patients in our research are approved by the ethics committee of the Getafe University Hospital (Madrid, Spain) and is conducted in accordance with the Declaration of Helsinki of the World Medical Association. All participant patients have signed an informed consent.

- Subjects Totally 40 patients with different levels of essential tremor were involved in the experiments. Part of them were under tremor therapy, such as primidone, propranolol and clonazepam. Medication for ET was maintained during the experiment. In order to 


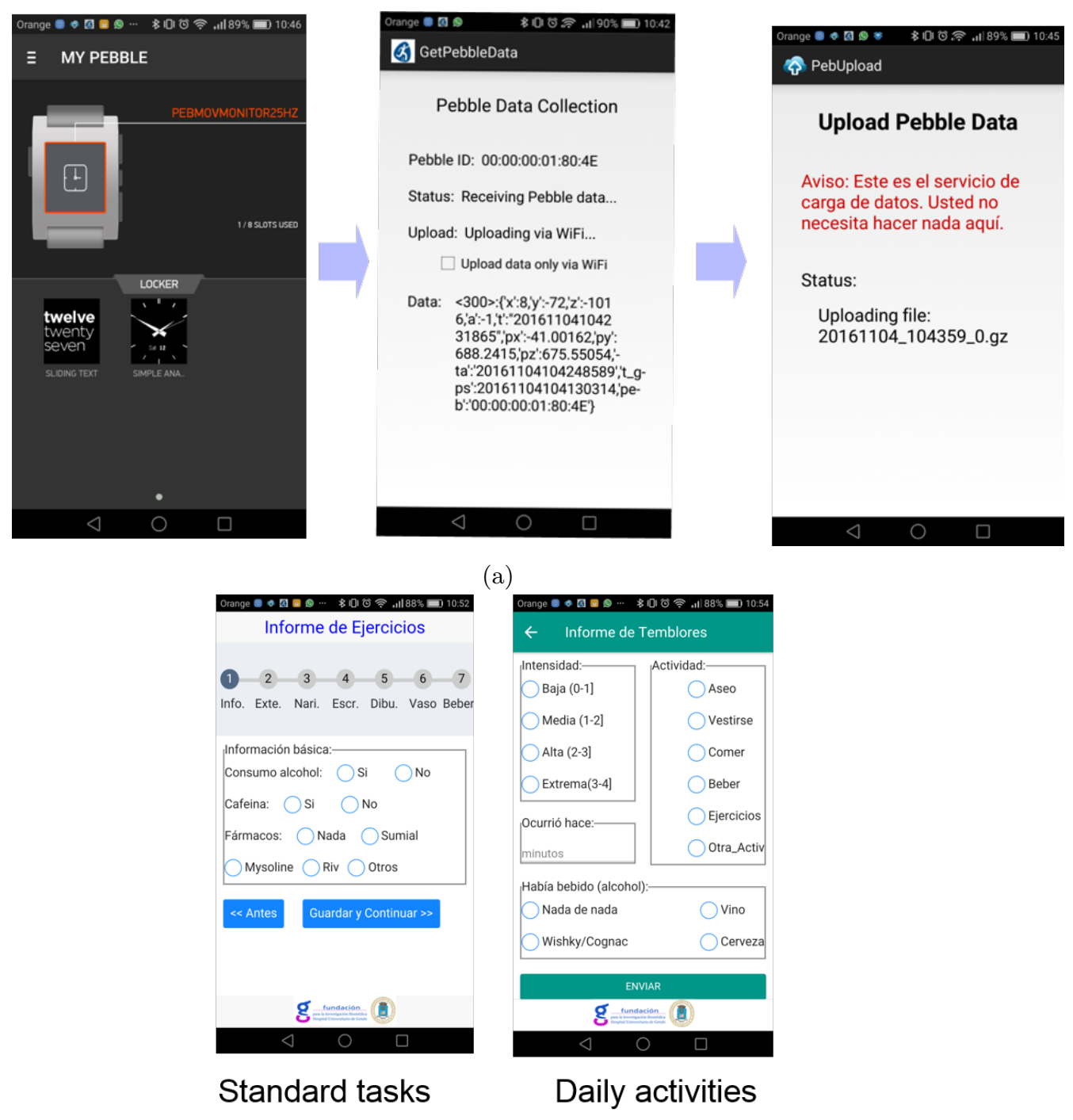

(b)

Figure 2.3: UIs of APPs running in smartphones

compare, the data five healthy subjects were also included.

- Procedures of experiment In general, the experiments include two phases and the procedures are different. The first phase of the experiment focused on the long-term monitoring of ET, so the data collecting process involves both inside and outside of hospital. At the beginning, each patient needs to visit the hospital and realize a series of FTMTRS tasks 37 under the supervision of a neurologist from the research team. The tasks include (1) keeping hands relaxed on the lap for evaluating rest tremor, (2) holding their arms extended horizontally for studying postural tremor, and (3) repeating finger-nose test several times, writing "this is a sample of my writing," signing, dating, drawing two spirals and a line between two points and two bars, and pouring a full glass of water into another empty one several times for examining kinetic and intentional tremor. All tasks are performed during 15 seconds except writing and drawing. Each item is rated on a scale from 0 to 4 corresponding to the severity of tremor by the patient and by two neurologists. 
All patients have a fully documented guide containing clear instructions on how to perform and quantify each task. When performing the standard tasks, the patients were asked to report a self-evaluation score for each task and the system would add a timestamp for each task, so that the task-induced tremor can be identified by comparing the timestamp. During these tasks, all movement data are being collected and uploaded to the remote server in real time. The out-hospital patient monitoring consists of continuous capturing of all unrestricted movements since waking up in the morning until bedtime at night and during the predefined tasks performed three times every day. Patients should perform and rate all tasks according to FTMTRS, oriented by a fully documented guide.

The second phase of the experiment focused on the accurate evaluation of tremor levels taking into account the background activities when the tremor appeared. Therefore, only standard tasks were performed inside the hospital. The standardized experiment was designed on the basis of the FTMTRS tasks. The repetition numbers of the tasks were mainly based on the duration time of each task and each repetition. The target was to keep the overall duration of each task more or less the same while considering the body conditions of the patients to avoid muscle fatigue. Under the supervision of at least two neurologists, and wearing the watch firmly on the more affected wrist, patients perform the standardized tasks guided by a vided ${ }^{1}$. The tasks include:

(1) Keep both arms horizontally extended for 20 seconds and then relaxed on the lap for 10 seconds. This task is repeated three times.

(2) Repeat the finger-nose test (touching the nose with the index finger with the arm raised to 90 degrees) 10 times, and then keep the hands relaxed on the lap for 10 seconds. This task is repeated twice.

(3) Write "THIS IS A SAMPLE OF MY WRITING", and then keep both hands relaxed on the lap for 10 seconds. This task is repeated three times.

(4) Draw a large and a small spiral, and three lines sequentially with a 10 seconds rest between every two drawings. This task is repeated twice.

(5) Pour a full glass of water into another empty cup and then pour back for three times, and then rest for 10 seconds. This task is repeated three times.

(6) Simulate the action of drinking a full glass of water five times and then resting for 10 seconds. This task is repeated twice.

The experiments were videotaped for subsequent analysis and tremor scoring by two neurologists in a blind way. Each item was rated on a scale from 0 to 4 (no tremor to severe).

\footnotetext{
${ }^{1}$ Available online: https://www.researchgate.net/publication/325923937
} 


\subsection{Data analysis algorithms}

\subsubsection{Hardware and software}

All data preprocessing and model training in this study were implemented with a computer equipped with a 4-core Intel Core i5-4460 $3.2 \mathrm{GHz}$ CPU and a $12 \mathrm{~GB}$ of RAM memory. The operating system is Ubuntu Linux 16.04 64-bit version. Data preprocessing is performed with RStudio. The deep learning model training and testing are conducted with TensorFlow (Version 1.0) and the model was built in Python (Version 2.7) language. More specifically, the TF.Learn module of TensorFlow was adopted for creating, configuring, training and evaluating the deep learning model. TF.Learn is a high-level Python module for distributed machine learning inside TensorFlow. It integrates a wide range of state-of-art machine learning algorithms built on top of TensorFlow's low level APIs for small to large-scale supervised and unsupervised problems.

\subsubsection{Tremor identification}

As introduced in previous sections, the frequency of ET is between 4 and $12 \mathrm{~Hz}$, which is much higher intended human actions. Therefore tremor can be identified according to the frequency difference. Fast Fourier Transform (FFT) can be used to transfer the raw acceleration data from time domain to frequency domain. After the transformation, relevant frequencies are filtered to check if amplitudes above certain threshold between 4 and $12 \mathrm{~Hz}$ exist.

Since the amplitude can only reflect the information of each corresponding frequency, the tremor energy in this frequency range can be calculated to better represent the complete information. According to the energy in this range, we classified tremor into four levels corresponding to the FTMS scale using K-means clustering algorithm. Then we compared the clustering result with the evaluation scores from the two neurologists and the self-evaluation results from the ET patients. The correlation analysis results proved the feasibility of our approach. With this approach, we can monitor ET patients' tremor status remotely, in real-time and long-term, which may help neurologists better understand patients' situation and improve treatment correspondingly.

This part of research has been published in the journal Frontiers in Neurology. More details about tremor identification, clustering and correlation analysis are introduced in this paper. The full text is included in the following chapter.

\subsubsection{Activity recognition}

With the above-mentioned approach, it is possible to understand patients' general tremor situations. However, it is not enough in practical applications, because even for the same patient, the tremor level varies a lot during the day when the patient doing different activities. It is important for the neurologists to know not only when serious tremor appears, but also which activity caused it. For this purpose, we extended our research to Human Activity Recognition 
(HAR) using machine learning techniques.

Deep learning has shown outstanding performance in HAR based on motion sensor data. Data preprocessing is an important part of deep learning projects and takes up a large part of the whole analytical pipeline. Data segmentation and data transformation are two critical steps of data preprocessing. In order to find the best algorithm for our purpose, we analyzed the impact of segmentation methods on deep learning model performance, and compared four data transformation approaches. The comparison was conducted involving several public datasets and our own data. The multichannel method, which treats the data for the three axes as three overlapped color channels, produced the best performance.

This part of research has been published in the journal Sensors. More details about the data preprocessing approaches, deep learning models and the activity recognition performances are introduced in this paper and the full text is included in the following chapter.

\subsubsection{Activity-aware tremor evaluation}

By converging our previous studies about tremor identification and activity recognition, we finally proposed an activity-aware ET evaluation method using deep learning based on acceleration data. Two types of deep CNN models were built for activity classification and tremor level evaluation. Therefore, the ET diagnosis process not only focuses on tremor itself, but also takes into account the background activities when tremor occurred. A correlation analysis between the proposed activity-aware tremor evaluation results and the ratings of neurologists was performed. Stronger correlation was found than that obtained in previous studies, which didn't consider the impact of background activities. It means that the introducing of activity classification can increase the accuracy of tremor severity evaluation against the traditional non-activity-aware models.

This part of research has been published in the journal Parkinsonism and Related Disorders. More details about the activity classification models and tremor evaluation models and their performance during the experiments are introduced in this paper. The full text is included in the following chapter. 


\section{Chapter 3}

\section{Collection of publications}

\subsection{Publication 1}

- Title: The development of intelligent healthcare in China

- Authors: Xiaochen Zheng, Carlos Rodríguez-Monroy

- Publication date: 2015/5/1

- Journal: Telemedicine and e-Health

Impact Factor: 2.165 (2017) / 2.418 (5 year)

Quartile: Q2

ISSN: $1530-5627$

Publisher: MARY ANN LIEBERT, INC, NEW ROCHELLE, NY 10801 USA

- Volume: 21

- Issue: 5

- Pages: 443-448

- DOI: https://doi.org/10.1089/tmj.2014.0102

- Copyright statement: This study is published in subscription journals. The copyright of the postprint manuscript (accepted version after peer review) belongs to the publisher. According to the policy of the publisher, "Authors may archive their preprint manuscripts (version prior to peer review) at any time without restrictions ${ }^{1}$, Therefore, the preprint version of the article is included in this thesis and the final publication is available from Mary Ann Liebert, Inc., publishers https://doi.org/10.1089/tmj.2014.0102.

\footnotetext{
${ }^{1}$ https://home.liebertpub.com/publications/telemedicine-and-e-health/54/for-authors
} 


\title{
The Development of Intelligent Healthcare in China
}

\author{
Xiaochen Zheng, MSc, and Carlos Rodríguez-Monroy, PhD \\ Escuela Técnica Superior de Ingenieros Industriales, Universidad Politécnica de Madrid
}

\begin{abstract}
Background: Intelligent Healthcare (IH) is proposed with the fast application of Internet of Things (IoT) technology in healthcare area in recent years. It is considered as an expansion of eHealth and Telemedicine. As the largest developing country, China is investing large amounts of resources to push forward the development of IH. It is one of the centerpieces of the Chinese New Healthcare Reform and great expectation is placed on it to help solve the conflict between limited healthcare resources and the large patient population. Materials and Methods: Essential policies, milestones, standards and specifications from the Chinese government since 1990s were reviewed to show the brief development history of IH in China. Some typical cases and products have been analyzed to present the current situation. The main problems and future development directions have been summarized. Conclusions: The IH industry in China has great potential and is growing very fast, but a lot of challenges also exist. In future both the government support and the active participation of non-governmental capital are needed to push forward the whole industry.
\end{abstract}

Key words: intelligent healthcare, China, telemedicine, e-health, mobile healthcare

\section{Introduction}

The information technology revolution which started last century has made the world totally different and the healthcare industry has also entered a new era. Within the past decades, a variety of new concepts gradually emerged in the healthcare area, such as Telemedicine ${ }^{1}$, Digital Hospital $^{2}$ and E-health ${ }^{3}$ etc. In recent years, a new terminology - Intelligent Healthcare (IH) - is spreading gradually due to the rapid growth of information technologies.

There is still no formal definition about IH in the academic field, although this term is found frequently in healthcare documents published by the Chinese government and by Chinese healthcare companies. In our opinion, IH is a new stage of information technology application in the healthcare field. It is developed on the basis of regional healthcare information platforms. Supported by Internet of Things (IoT) ${ }^{4}$ technology, healthcare services can be delivered to any individual at any time and in any place. Current technologies, like Telemedicine, Digital Hospital and E-health, will be foundations and essential components of IH.

Currently, IH is still in the initial stage and there are still many obstacles before its wide application. Many governments and companies have invested large amounts of resources in this area, as it is growing to become a huge market and it would be an effective method to solve many problems in the current healthcare systems. As the largest developing country, China is also making great efforts in this area. 
In this paper, we review the current situations of IH in China, illustrated with examples; some of the main challenges ahead are analyzed and future development trends are considered.

\section{Actions by the Chinese Government}

Since the Chinese government implemented the Reform and Opening Policies in 1978, its economy has been growing so quickly that it has become the second largest economy in the world. However, the living conditions of the Chinese people are not at the corresponding level. A large gap exists between the development of economics and people's wellbeing, like healthcare, education, environment and so on $^{5}$. Among these, the healthcare issue is turning into a serious conflict in society. Expensive medical bills and difficult access to adequate medical services have been an issue of concern in China recently.

The root cause of this problem is the lack of medical resources and the uneven distribution of these resources. The population of China, which is more than 1.3 billion, accounts for more than $22 \%$ of the world population, but its healthcare investment only makes up a $4.5 \%$ portion of the world and the medical staff represents only $13 \%{ }^{6}$. Making matters worse, nearly $80 \%$ of these scarce medical resources are centralized in large cities and $80 \%$ of them in these cities are located in a few large hospitals ${ }^{7}$. The clinical facilities in communities and rural areas get little investment and they are seriously lacking medical staff and equipment. This kind of ill-balanced distribution crippled the function of small community clinics. To solve this problem, the Chinese government is pushing forward the New Healthcare Reform and IH is an important part of it.

In March of 2009, the Opinions of the Communist Party of China (CPC) Central Committee and the State Council on Deepening the Healthcare System Reform (the Opinions) ${ }^{8}$ was published. For the first time, the construction of IH system is emphasized in an individual section in such an important national reform plan. In section fourteen, four targets of the development of Intelligent Healthcare were proposed: "build a practical, medical information sharing system", "push forward the healthcare information standardization", "establish a public healthcare service platform" and "construct Telemedicine network in rural and remote areas" ${ }^{\text {. }}$. These policies have stimulated the development of IH in different aspects: the efficiency of sharing information among different regions and the capability of disease control and prevention have been greatly improved; the healthcare record system covering a large population has been built: most large hospitals have established their information systems and the telemedicine networks also spread very fast in rural areas.

In fact, the preparation of IH began much earlier. Dating back to 1990s, some healthcare institutions had built their own electronic registration and invoicing systems to improve the work efficiency. After the devastating flood of 1998, the construction of a national disease control and prevention system was started by the government. The National Health Information Network project, with a budget of 230 million RMB (37 million USD or 27 million Euros), was approved in July 1999 and started the following year'. By the end of 2002, a hierarchical health information network covering 2,861 counties, 333 cities and 31 provinces was built which played a very important role in the battle against the Severe Acute Respiratory Syndrome (SARS) in $2003^{10}$. In March 2003, the Ministry of Health published the National Development Programming Compendium for Health Information Construction (2003-2010) ${ }^{11}$. In the following years, the government invested more resources to improve the national health information system, and 
started the construction of preventive healthcare information systems, healthcare service information systems, health surveillance information systems, community healthcare service platforms and so on. According to the plan of the Ministry of Health, by the end of 2011, 30\% of rural population and $50 \%$ of urban population would have personal health records, and in 2020, a health record system covering all Chinese residents is supposed to be created. ${ }^{12}$ In practice, by 2012, 900 million residents had their personal health records and more than 50\% of them had digital records. ${ }^{13}$ These information systems enabled the foundation for the development of IH in China.

At the national level, the Ministry of Health has been leading the development of IH. In late 2009, the Basic Architecture and Data Standards for Electronic Medical Records (tentative) ${ }^{14}$ and the Regional Health Information Platform Construction Guide Based on Health Records ${ }^{15}$ were published. During the following years, more standards were built, such as Rules of Healthcare Data Element Standardization ${ }^{16}$, Classification and Coding Rules of Healthcare Information Datasets ${ }^{16}$, Basic Datasets of Urban and Rural Residents' Healthcare Records ${ }^{17}$, Specification of Healthcare Information Basic Dataset ${ }^{18}$ and so on. These policies greatly improved the standardization of healthcare information in China, which is the basis for the development of IH.

Together with the policies, substantial capital from the government has been invested to support the development of IH. In early 2009, after the publication of the Opinions document, an investment of 850 billion RMB (137 billion USD or 100 billion Euro) has been spent to implement the healthcare reform till the end of 2011, in which more than 20 billion RMB (3.2 billion USD or 2.4 billion Euro) has been spent in the IH area ${ }^{19}$. In 2012, another investment of 61 billion RMB (10 billion USD or 7.2 billion Euro) was planned to improve the level of $\mathrm{IH}^{20}$. These investments have greatly enhanced the development of IH in China. The annual growth rate of the $\mathrm{IH}$ market has been kept higher than $20 \%$ during the past 5 years ${ }^{21}$.

\section{Current Situation}

While the government is making efforts in pushing forward the development of general healthcare systems and their standardization, many Chinese enterprises and organizations are trying to solve some technical problems and make IH applicable in practice. They have made substantial achievements in the following aspects:

\section{Telemedicine Systems}

The development of Telemedicine in China started in the 1990s. A special medical satellite network, named Jinwei Network, was launched in $1997^{22}$. After nearly twenty years development, it has been widely applied and has formed a large market. Although a large part of the market is taken by a few large international companies, many local companies in China can also provide advanced Telemedicine solutions now, represented by SUNPA ${ }^{23}$, Huawei ${ }^{24}$, Neusoft $\mathrm{ft}^{25}$, and Kingway $^{26}$ among others. Their Telemedicine Solutions cover nearly all aspects of the telemedicine industry, including interactive tele-consultation systems, medical image reading systems, medical image acquisition and digital conversion systems, dynamic and static graphic image inspection systems, microscopic image inspection systems, surgical guidance systems, distance education systems, etc. As an example, SUNPA Corporation has one of the largest telemedicine networks in the world, serving more than 490,000 telemedicine links, with 5,000 
experts, 50,000 collaborating physicians and 700 professional technicians ${ }^{26}$. Taking advantage of the large market in China, these companies are growing fast.

\section{Hospital Information Systems}

In the past decade, the development of hospital information systems in China has been very rapid, especially after the start of new healthcare reform in 2009. Similarly with telemedicine, there are many local software companies providing hospital information solutions. The difference is that a large number of hospitals built their information systems based on their own standards ${ }^{27}$, and founded many small software companies. This created obstacles for regional or even national system integration in future. A typical hospital information system includes several modules, such as Hospital Information System/Clinical Information System (HIS/CIS), Laboratory Information Systems (LIS), Electronic Medical Record (EMR), Picture Archiving and Communication Systems/Radiology Information System (PACS/RIS). Many companies in China, for example, Neusoft ${ }^{28}$ and Huawei ${ }^{29}$, have the ability to provide such solutions.

With the explosive growth of cloud computing, many software suppliers are providing integrated systems supported by this technology. Figure 1 shows the typical structure of a hospital information system integrated with cloud computing.

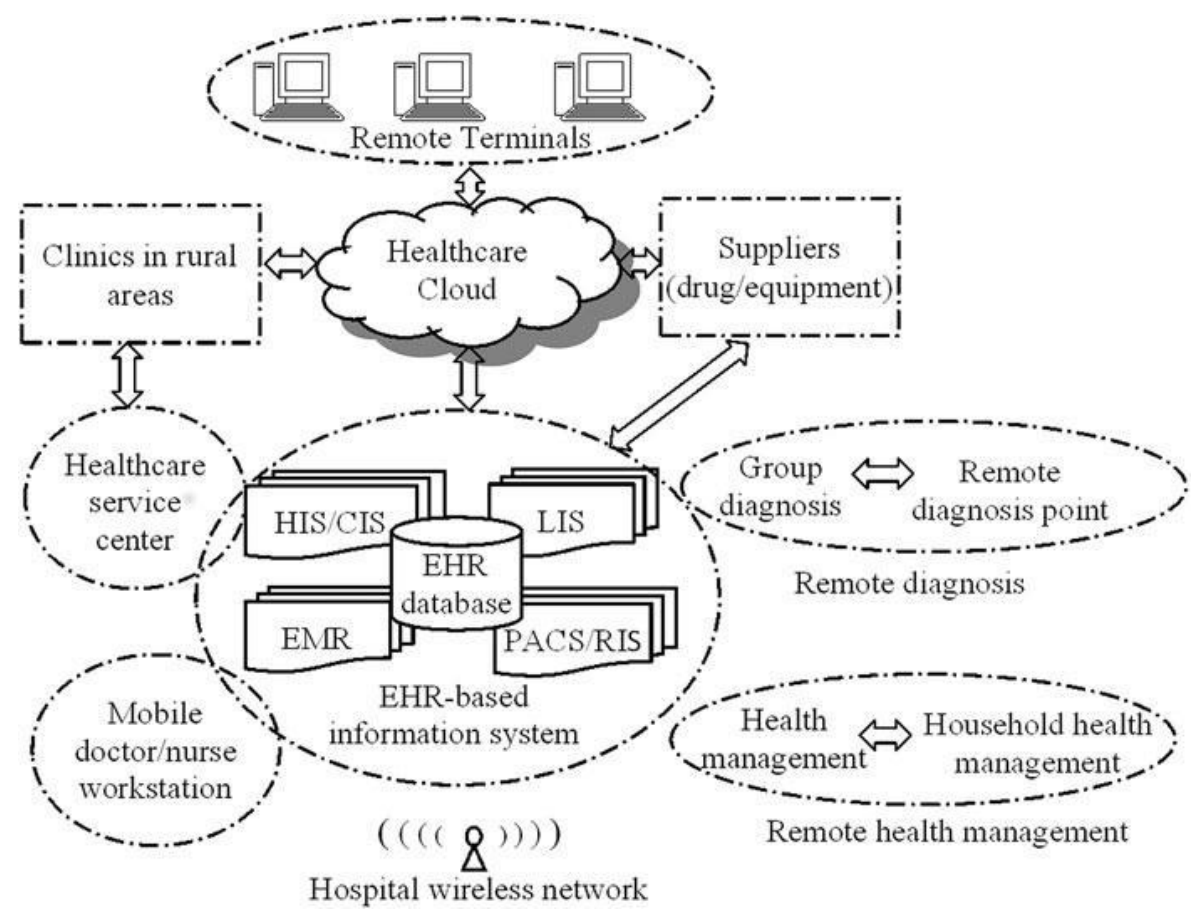

Figure 1 Structure of a hospital information system

\section{Regional Healthcare Information Platforms}

The Regional Healthcare Information Platform (RHIP) is a higher level of hospital information system. It can help solve the problem of "information isolated island" among hospitals. In many more developed areas of China, the local governments have been building such platforms to balance the distribution of medical resources and some of them have already been taking advantage of these platforms. Take Minhang as an example, which is a district of Shanghai, the second largest city in China. It started to build an Electronic Health Record (EHR) system in early 
2008 as the first testing prototype of China. Then a RHIP connected with more than 200 hospitals and organizations covering at least 2 million people was built. More than $93 \%$ of the resident population and $30 \%$ of the floating population have their EHRs. Based on this EHR system, they integrated existing healthcare information resources into the RHIP, consisting of 34 modules, such as chronic disease management, early cancer detection, women and child healthcare, immunization, remote consultation, drug management and so on $^{30}$. Currently, many other cities in China are building such kind of information platforms.

\section{Mobile Healthcare and Wearable Devices}

Since 2012, several new mobile devices are coming out continuously, like smart watch, intelligent bracelet, intelligent glasses and so on. In near future, these devices will be used to collect data from humans and transfer them to central cloud computing servers through smart terminals, so that the doctor can get the status of the patient from anywhere at any time. According to a research report ${ }^{31}$ by iiMedia Research Group, a third-party research agency in Hong Kong, focusing on mobile Internet data mining and integration marketing, the Chinese market will gradually become the core of the global market for wearable devices. Various forms of wearable devices will access people in all aspects of life. In 2012 the mobile healthcare equipment market reached 70 million US dollars and is expected to exceed 170 million US dollars by $2015^{31}$. With the whole wearable device market gradually warming up, mobile healthcare will also achieve rapid development and expand even faster as people's concern for their own health is increasing.

\section{Open Challenges}

Generally, the development of IH in China is now moving from hospital to regional level information systems. There is still a long way to go and many obstacles appear ahead:

(1) Lack of a clear top design for the development of IH. As a new industry, IH has both the properties of high-tech industry and the background of public healthcare administration. Its development requires a standardized, healthy and well-designed environment. However, currently there is still no clear and formal plan of the whole industry, while the current plans are only describing the prospect of IH without a practical strategy for implementation, schedule or methodology. This has resulted in an unbalanced and sometimes duplicated growth in different areas. For instance, many hospitals are building their information systems according to their own standards, which is not only a poor use of resources but it also introduces barriers for future higher level integration.

(2) Lack of unified rules and industry standards for intelligent healthcare platforms. Unlike some developed countries, the development of healthcare information in China started late and lacks a history of data accumulation. The Electronic Healthcare Record System is still under construction and the information can hardly be shared among different areas. The standard for data sharing is still not complete, although the government has published a series of standards. There is no unified terminology database currently, which is another huge obstacle for the development of IH. Besides, many technical problems have not been solved, such as intelligent collecting, transferring, processing and sharing of data, etc. It is important to avoid the creation of "isolated information islands" which has happened in many other industries. 
(3) Existing medical institutions are not keen on providing IH services. There is no doubt that IH will benefit the whole society. However, for their own interests, a large proportion of hospitals are not enthusiastic about building IH platforms and supplying IH services. One reason for this is that $\mathrm{IH}$ can release the pressure of some high level large hospitals by sending part of their patients to lower level hospitals. But the profits of the former ones will also shrink. It is common in China for hospitals to make profits by selling medicines with high prices. If IH is widely used, patients can buy medicines from any pharmacy and hospitals will then lose a significant part of their income. The high cost of building $\mathrm{IH}$ basic platforms and service systems is another reason. Facing the pressure of too many patients, they prefer to spend the limited financial resources in upgrading infrastructures, purchasing new equipment and training and recruiting staff. Besides, the wide use of $\mathrm{IH}$ needs experienced doctors to handle the large amount of data from the end users, whilst most of the medical staff in large hospitals now are already overburdened. Even though IH may help relieve the pressure, the decision makers are not willing to take the risk of investing part of the scarce medical resources into IH before the benefits are confirmed.

(4) Investors are not willing to invest in $\mathrm{IH}$. The development of IH requires a large amount of upfront investment, but the payback period is so long that investors are reluctant to get involved $^{32}$. With the fast growth of the economy in China, a large amount of private capital has accumulated in the past two decades. The owners of this capital are looking for investment opportunities all over the world. If part of this money can be invested in the $\mathrm{IH}$ area, it will create great power to push this industry forward. But healthcare is a special market and the New Healthcare Reform is still under way. Additionally, the future of IH is not totally clear. Investors are hesitating to enter this area.

(5) Disordered competition in the supply side of the market with too many small companies. During the past few years, hundreds of small companies appeared in the healthcare information market. According to a report by National Healthcare Information Technology Alliance, the number of such kind of companies was more than 400 by July $2013^{33}$. Most of them were founded based on one or two local hospital information projects and their business is limited to a certain area. Only a few of them can provide regional information systems. The platform structure and adopted standards differ from each other, making future integration difficult. It also dispersed the limited resources which is also an obstacle for the integral development of the whole industry.

\section{Future Development Directions}

The IH industry in China has been growing very fast since the New Healthcare Reform from 2009 and it is expected to keep growing in the coming years. The government, healthcare institutions and solution suppliers are all focusing on this area. Some aspects should be enhanced in the future:

(1) Complete the top design for IH, establish detailed standards and specifications. A clear and implementable development framework from the government is the urgent need for the $\mathrm{IH}$ industry in China. It is the guidance for all the remaining tasks. It is also the government's responsibility to organize a special expert team integrated by representatives from government, healthcare institutions, medical scholars, software solution suppliers etc., in 
order to accelerate the establishment of national standards and specifications. This would be a huge task and only the central government has the power to organize it in today's China.

(2) Keep on pushing forward the construction of regional healthcare information systems and implement data sharing nationwide. In some developed areas, mainly large cities, regional healthcare information platforms have already been established. But development is unbalanced within the country, especially in the western part of China and also in large rural areas, where the pace is much slower. In the coming years, more resources should be spent in these areas to narrow the gap. The investment should include not only hardware platforms, necessary equipment and software systems but also human resources. Efforts should be made to encourage more medical staff and experienced technicians to go to rural areas and communities.

(3) Learn from successful cases in other countries. There are many successful cases of IH industry in some developed countries and regions, like the United States, Western Europe and Japan. To learn experiences from them would be a shortcut for China, while taking into account the different situations. China has a much larger population and the technical base is not as developed as in these advanced regions. Since China is facing the challenge of fast urbanization and population aging currently, the development of $\mathrm{IH}$ should be combined with the entire healthcare reform.

(4) Improve the market environment to attract private capital into healthcare area. Private investment has a great potential to promote the $\mathrm{IH}$ industry but now it stays away from this industry. Investors are hesitating mainly because the future of IH is still uncertain and there are no clear policies and laws to guide and regulate its development. The government should publish more favorable policies, like absorbing private healthcare institutions into the national insurance system, simplifying the approval procedures, and completing medical staff management mechanisms to change the perceptions and reduce the reluctance of investors.

(5) Support some software companies to solve key technical problems Software suppliers are also very important for the development of IH. In fact, they can take the role of leading the technical development of IH. They are the architects of final information platforms and they know well what the customers need and where the weak points lie. However, few companies in China are willing to take the risk to do basic research, as they do not have enough resources like large multinational corporations do. In this situation, the government should support some selected companies to do basic research and to lead the development of IH in China.

\section{Conclusions}

The development of $\mathrm{IH}$ is still in its infancy with great potential in future. China has put enormous efforts to promote the growth of this industry and has made some relevant achievements. At the national level, the government is trying to improve the whole healthcare system. A series of standards and specifications has been published, and some basic information sharing networks have been established. On the other side, hundreds of local companies have been growing very fast and some of them can provide advanced technologies, from smart hospitals to regional 
healthcare system. The mobile healthcare based on wearable devices is exploding in China and it is possible to become the largest market in the world. Meanwhile, many serious problems remain due to the insufficient technical development and unbalanced distribution of healthcare resources. To overcome these challenges, more support from government and social institutions is necessary.

IH is a complex industry and the purpose of this paper is to briefly introduce the general situation in China. More detailed and technical issues need to be analyzed in the future. For example, the influence of the social insurance system reform on the development of IH, the roles of Chinese government and private investments in IH development and their relationships, the IH current situation comparison between China and other nations, the assimilation of best practices in advanced countries etc.

Under the background of New Healthcare Reform, an enormous amount of public and private resources are spent for IH development in China. It will become an even more important topic in the coming topic in the following years as many new opportunities and challenges are appearing.

\section{Acknowledgements}

This work is financially supported by the China Scholarship Council. 


\section{References}

1. Sanjay S, Victor M, Shakhina J, Reena D, Charles RD, Nupur P, Ronald CM. What is Telemedicine? A collection of 104 peer-reviewed perspectives and theoretical underpinnings. Telemedicine and e-Health. October 2007, 13(5): 573-590.

2. Lynne AK, Fisher JE, Laura J, Zeltwanger PE. The digital hospital: opportunities and challenges. Journal of healthcare information management: JHIM 2002;17(1):37-45.

3. Hans Oh, Carlos R, Murray E, Alejandro J. What is eHealth (3): a systematic review of published definitions. Journal of medical Internet research 2005;7(1).

4. International Telecommunication Union. ITU Internet reports 2005: the Internet of Things. Technical report, Geneva, November 2005.

5. Xiaozong W. The GDP of China became second of the world but many industries still lagging far behind. China Economic Weekly, March 2011.

6. Yan Y. The distribution of healthcare resources in China is still unbalanced. Health News, July 2013.

7. Xiaoyu W. China's medical and healthcare system reform situation and countermeasures, January 2007. Available at http://http://www4.zzu.edu.cn/tzhb/tzv2/show.aspx?id=442 (last accessed March 14, 2014)

8. State Council of China. Opinions of the CPC Central Committee and the State Council on deepening the healthcare system reform, March 2009.

9. National Health and Family Planning Commission of China. Provisions about national healthcare information network construction project, January 2001.

10. Ming L. Intelligent healthcare development in China: situation, problems and countermeasures. Master Thesis, University of Science and Technology of China, Hefei, China, May 2011.

11. Ministry of Health of China. National health information development plan (2003-2010), March 2003.

12. Ministry of Health of China. Guidance of Ministry of Health on urban and rural residents' health records management. December 2009.

13. China News. The digitalization of Chinese healthcare industry speeds up, half population got health records. February 2012. Available at http://finance.chinanews.com/jk/2012/0227/3701936.shtml (last accessed July 4, 2014)

14. Ministry of Health of China. Basic architecture and data standards for electronic medical records (tentative), December 2009.

15. Ministry of Health of China. Guide for construction of regional health information platform based on health records, May 2009.

16. National Health and Family Planning Commission of China. Publish announcement of 4 mandatory health standards, "Rules of healthcare information data element standardization" etc. (notice [2009], no.3), January 2009.

17. National Health and Family Planning Commission of China. Publish announcement of 35 mandatory health standards, "Catalog of healthcare information data elements" etc. (notice [2011], no.13), August 2011.

18. National Health and Family Planning Commission of China. Publish announcement of 23 
mandatory health standards, "Specifications of healthcare information basic dataset" etc. (notice [2012], no.5), March 2012.

19. State Council of China. Key plans of the Healthcare System Reform in recent years (20092011). March 2009.

20. Ministry of Health of China. "Healthy China 2020" strategic study report". August 2012.

21. Shigao Dong. "Healthy China 2020" 400 billion investment push forward the development of healthcare system. August 2012. Available at http://www.qianzhan.com/analyst/detail/220 /120824-23cf0c2c.html (last accessed March 14, 2014).

22. Guiqiu J, Kejing Z, Daxiang C. Analysis of current situations and future applications of telemedicine. Medical Journal of National Defending Forces in Southwest China 2001;3(11):211213.

23. SUNPA Enterprise. SUNPA product and service introduction: SUNPA telemedicine solutions. Available at http://www.sunpaimage.com/InfoCenter/ProductTec/ (last accessed March 14, 2014).

24. Huawei Enterprise. Introduction to Huawei telemedicine solutions. Available at http://enterprise.huawei.com/cn/solutions/trade/medical/theme/hw-143594.htm (last accessed March 14, 2014).

25. Neusoft Enterprise. Introduction to Neusoft telemedicine solutions. Available at http://www.mednet2000.com/medit/cn/product/ (last accessed March 14, 2014).

26. Kingway Enterprise. Introduction to Kingway telemedicine solutions. Available at http://www.kw120.com/ (last accessed March 14, 2014).

27. Junping Z, Zhenjiang Z, Huayuang G, Yi L, Wanguo X, Lianzhong R, Yunqi C, Shifu C, Xiaoyu Z. E-health in China challenges, initial directions, and experience. Telemedicine and eHealth. April 2010, 16(3): 344-349.

28. Neusoft Enterprise. Introduction to Neusoft digital hospital solutions. Available at http://medical.neusoft.com/solutions/1424/ (last accessed March 14, 2014).

29. Huawei Enterprise. Introduction to Huawei "Smart hospital" solution. Available at http://enterprise.huawei.com/en/solutions/trade/healthcare/numeral-hospital/hw-261613.htm (last accessed March 14, 2014).

30. Nan J. Shanghai Minhang built a new regional healthcare information platform, April 2010. Available at http://solution.hc3i.cn/art/201004/2585.htm (last accessed March 14, 2014)

31. iiMedia Research Group. China wearable device market research report. Technical report, Hong Kong, China, September 2013.

32. Xin Y. Push forward the development of intelligent healthcare in Shanghai. National People's Congress of Shanghai, 2011;10:26- 27.

33. National Healthcare Information Technology Alliance of China. Name list of healthcare information technology companies in different provinces and their contacts, July 2013. Available at http://www.chisc.net/baike/ylxxhgs/2011-03-22/28.html (last accessed March 4, 2014) 


\subsection{Publication 2}

- Title: Continuous Monitoring of Essential Tremor Using a Portable System Based on Smartwatch

- Authors: Xiaochen Zheng, Alba Vieira Campos, Joaquín Ordieres-Meré, Jose Balseiro, Sergio Labrador Marcos, Yolanda Aladro

- Publication date: 2017/3/15

- Journal: Frontiers in neurology

Impact Factor: 3.508 (2017) / 4.297 (5 year)

Quartile: Q2

ISSN: $1664-2295$

Publisher: FRONTIERS MEDIA SA, LAUSANNE, SWITZERLAND

- Volume: 8

- Pages: 96

- DOI: https://doi.org/10.3389/fneur.2017.00096

- Copyright statement: This article is published in Open Access journals. According to the publisher's policy, "Authors retain copyright of their work and can deposit their publication in any repository. The work can be freely shared and adapted provided that appropriate credit is given and any changes specified.' 2

\footnotetext{
${ }^{2}$ https://www.frontiersin.org/journals/neurology\#about
} 


\title{
Continuous Monitoring of Essential Tremor Using a Portable System Based on Smartwatch
}

\author{
Xiaochen Zheng ${ }^{1}$, Alba Vieira Campos ${ }^{2}$, Joaquín Ordieres-Meré', Jose Balseiro², \\ Sergio Labrador Marcos² and Yolanda Aladro ${ }^{2 *}$
}

'Department of Industrial Engineering, Technical University of Madrid, Madrid, Spain, ${ }^{2}$ University Hospital of Getafe, Getafe, Madrid, Spain

Introduction: Essential tremor (ET) shows amplitude fluctuations throughout the day, presenting challenges in both clinical and treatment monitoring. Tremor severity is currently evaluated by validated rating scales, which only provide a timely and subjective assessment during a clinical visit. Motor sensors have shown favorable performances in quantifying tremor objectively.

OPEN ACCESS

Edited by:

Pille Taba,

University of Tartu, Estonia

Reviewed by:

Aasef G. Shaikh,

Case Western Reserve University,

Andreas Sprenger

University Luebeck, Germany

${ }^{*}$ Correspondence:

Yolanda Aladro

yolanda.aladro@salud.madrid.oro

Specialty section: This article was submitted to

Movement Disorders,

a section of the journal

Frontiers in Neurology

Received: 18 October 2016

Accepted: 27 February 2017

Published: 15 March 2017

Citation:

Zheng X, Vieira Campos A

Ordieres-Meré J, Balseiro J,

Labrador Marcos S and Aladro Y (2017) Continuous Monitoring of Essential Tremor Using a Portable System Based on Smartwatch.

Front. Neurol. 8:96. doi: 10.3389/fneur.2017.00096
Methods: A new highly portable system was used to monitor tremor continuously during daily lives. It consists of a smartwatch with a triaxial accelerometer, a smartphone, and a remote server. An experiment was conducted involving eight ET patients. The average effective data collection time per patient was $26( \pm 6.05)$ hours. Fahn-Tolosa-Marin Tremor Rating Scale (FTMTRS) was adopted as the gold standard to classify tremor and to validate the performance of the system. Quantitative analysis of tremor severity on different time scales is validated.

Results: Significant correlations were observed between neurologist's FTMTRS and patient's FTMTRS auto-assessment scores ( $r=0.84$; $p=0.009)$, between the device quantitative measures and the scores from the standardized assessments of neurologists $(r=0.80 ; p=0.005)$ and patient's auto-evaluation ( $r=0.97 ; p=0.032)$, and between patient's FTMTRS auto-assessment scores day-to-day $(r=0.87 ; p<0.001)$. A graphical representation of four patients with different degrees of tremor was presented, and a representative system is proposed to summarize the tremor scoring at different time scales.

Conclusion: This study demonstrates the feasibility of prolonged and continuous monitoring of tremor severity during daily activities by a highly portable non-restrictive system, a useful tool to analyze efficacy and effectiveness of treatment.

Keywords: essential tremor, smartwatch, accelerometer, continuous monitoring, remote diagnosis

\section{INTRODUCTION}

Essential tremor (ET), the most prevalent tremor disorder, is a postural and kinetic tremor affecting $4.6-6.3 \%$ of adults above the age of 60-65 (1). It represents a family of diseases rather than a single one, which could be associated with gait disorders and cognitive impairments (2). Although ET is not a life-threatening disease, it has an important negative impact on all 
aspects of life quality, including social and psychological, due to their exacerbation in public (3). It mainly affects the upper limbs with the consequent interference in basic activities of daily living, such as handwriting, dressing, eating, and self-care (4). Progress in the treatment of ET is limited because of the poor understanding of many of the underlying conditions (5). Tremor analysis during daily living is crucial to better understand the patient status and to evaluate treatment effects (6). In clinical practice, it is usually assessed by several tremor validated rating scales (7-12). Although these have demonstrated clinical utility, they require the presence of a clinician for scoring. Their results are subject to clinical judgment and show significant intra- and inter-explorer variabilities. The main limitation is that they only provide an instantaneous, subjective, and qualitative assessment of tremor intensity during a clinical visit and do not allow extended continuous monitoring of tremor fluctuation patterns throughout the day or in home environments $(6,13)$.

Essential tremor shows a frequency between 4 and $12 \mathrm{~Hz}$ and variable amplitude, depending on stress level, position, voluntary movements, and disease evolution (14-17). It is higher than the frequency of normal voluntary human movements. Therefore, it is possible to detect tremor on the basis of the frequency difference $(3,13)$. The feasibility of classifying tremor according to the acceleration data collected from patient's wrist has also been reported (18). A variety of tremor data collecting systems based on acceleration sensors have been developed and applied in many studies $(6,19-27)$. However, most of these systems are designed in the laboratory environment and will cause inconvenience to the patient in case of long-term application. For example, the Kinesia ${ }^{\mathrm{TM}}$ (CleveMed) system has shown a good correlation with scale tremor scores, for all, rest, positional, and kinetic tremor $(6,28)$. Although this system is portable, it still limits the movement of the limb and it is hard to monitor the patient remotely due to the distance limitation. The development of wearable technology, such as smartwatch, smart band, and smart glasses, provides a new method to collect motion data more conveniently. The study presented in Ref. (29) has verified the practicability of using a smartwatch to analyze and diagnose tremor.

This research testifies to the feasibility and potential clinical utility of a new highly portable system capable of continuously recording arm motion data and sending them to a remote server through a mobile terminal in real time. The aim is to quantify the amplitude and frequency of the tremor and establish greater traceability with daily activities. The main device of the system is an Android smartphone and a smartwatch that contains a triaxial accelerometer. There is a database on a remote server, managing all the data collected from the patient without distance limitation. This system is able to monitor the patient's movement continuously without introducing much inconvenience. Accordingly, a quantitative overall situation of the patient in a long-range scenario is accessible.

\section{MATERIALS AND METHODS}

This study is approved by the ethics committee of the Getafe University Hospital (Madrid, Spain) and is conducted in accordance with the Declaration of Helsinki of the World Medical Association. All participant patients have signed an informed consent. The data collecting system only collects anonymous data from the smartwatch and the smartphone. Deidentified data were used, so that only the local investigator was aware of the source of the data and could associate them to a specific patient. Collected technical data are stored in a local server within the network of Technical University of Madrid protected by several firewalls. In addition, only authorized researchers of the team can access them. Therefore, patient privacy has been well protected throughout the study.

\section{Subjects}

Nine ET subjects, aged 60-77 years (mean 69.0 \pm 6.6 SD, six males and three females), with different levels of ET are included, all of them over the moderate level on Fahn-Tolosa-Marin Tremor Rating Scale (FTMTRS). The data of one patient are destroyed due to hardware problem (Android phone battery) and the data of the remaining eight patients are successfully collected, aged 60-77 years (mean 68.0 $\pm 6.3 \mathrm{SD}$, six males and two females). All patients selected showed both, postural and intentional tremor, although the tremor levels are different between patients and in each patient. Five patients were under tremor therapy, two were taking propranolol, another couple of them were on treatment with primidone, and the other one was taking clonazepam. The medication was maintained throughout the study. The data of a healthy male, aged 54 years are also recorded to compare with the data of the patients.

\section{Monitoring System}

A portable human movement monitoring system has been previously developed (30). This three-layer system is composed of a Pebble smartwatch, ${ }^{1}$ which contains a triaxis accelerometer and Bluetooth 4.0, for recording the user's arm movement data; an Android smartphone for receiving data from Pebble and uploading them to a remote server; and a cross-platform documentoriented NoSQL MongoDB database ${ }^{2}$ on remote server for data storage and analysis. The information collected using this system includes three-axis arm movement acceleration values. The feasibility of analyzing tremor using the data collected from a smartwatch has been validated in our previous work (31).

\section{Procedures of Data Collection}

At the first visit, each patient receives an initial training session to learn how to use the system (smartwatch and Android phone). Then, wearing firmly the watch on his/her wrist more severely affected (on the dominant hand if both are equally affected), the patient realizes the standardized tasks at hospital under the supervision of a neurologist from the research team. The tasks of FTMTRS (12) included are the following: (1) keeping hands relaxed on the lap for evaluating rest tremor, (2) holding their arms extended horizontally for studying postural tremor, and (3) repeating finger-nose test

${ }^{1}$ https://www.pebble.com/pebble-smartwatch-features (Updated May 2016).

${ }^{2}$ https://www.mongodb.com/ (Updated May 2016). 
several times, writing "this is a sample of my writing," signing, dating, drawing two spirals and a line between two points and two bars, and emptying a full glass of water into another empty one several times for examining kinetic and intentional tremor. All tasks are performed during $15 \mathrm{~s}$ except writing and drawing. Patients perform the tasks several times until they learn them. Each item is rated on a scale from 0 to 4 corresponding to the severity of tremor by the patient (not induced by the neurologist) and by two neurologists. In order to assure an adequate accomplishment of the standardized tasks at home, patients will have a fully documented guide containing clear instructions on how to perform and quantify each task. When performing the standard tasks, the patients were asked to report a self-evaluation score for each task and the system would add a timestamp for each task, so that the task-induced tremor can be identified by comparing the timestamp. During these tasks, all movement data are being collected and uploaded to the remote server in real time.

The movement monitoring process begins once the patient is able to manage the system and perform the tasks correctly. The outpatient monitoring consists of continuous capturing of all unrestricted movements since waking up in the morning until bedtime at night and during the predefined task performed three times every day. Patients should perform and rate all tasks according to FTMTRS, oriented by a fully documented guide.

\section{Data Analysis}

Three ratings of ET were performed on each patient: the assessment of neurologists during standardized tasks at hospital, the self-assessment of the patients during standardized tasks at home, and the analysis result based on the data collected during continuous monitoring. Since each patient performed standardized tasks three times per day and the monitoring lasts for several days, the average value of the patient's selfassessment scores of all tests for the same patient is calculated to represent the final score of patient's self-assessment result. Pearson correlation was used to evaluate the agreement degree between different scores.

\section{Tremor Identification}

Tremor was identified according to the frequency difference between voluntary movements and ET. From previous studies, it is known that the frequency of ET is between 4 and $12 \mathrm{~Hz}$. The tremor of the patients in this research shows a lower frequency, between 4 and $8 \mathrm{~Hz}$. We used a frequency filter of this range to identify tremor and eliminate intended human actions, whose amplitude is usually higher than tremor. When analyzing data collected from a healthy subject, infrequently we found high frequency activities that are easy to identify according to this difference of amplitude. Fast Fourier Transform (FFT) can be used to transfer the raw acceleration data from time domain to frequency domain. After the transformation, relevant frequencies are filtered. Therefore, the method is to cast the collected data into the frequency domain through FFT and check if amplitudes above certain threshold between 4 and $8 \mathrm{~Hz}$ (31). All movement data were collected with a frequency of $25 \mathrm{~Hz}$; therefore, after the FFT operation, the highest reliable frequency was $12.5 \mathrm{~Hz}$. This sampling rate is enough for the analysis of tremor which is lower than $12 \mathrm{~Hz}$. To see details, the reviewer or the readers can consult the original data at https://drive.google.com/file/d/0B3UOl_ J6yg0vOU8wcnhQVWlmYkk/view, where some figures show the original acceleration data and corresponding FFT results of the healthy subject and one of the patients.

\section{Tremor Classification}

The acceleration data collected on regular time basis were transformed into the frequency domain with the FFT operation. After the transformation, the frequencies between 4 and $8 \mathrm{~Hz}$ were filtered. Since the amplitude can only reflect the information of each corresponding frequency, the tremor energy in this frequency range was calculated to better represent the complete information.

Suppose $a(t)$ is the acceleration at the time $t$ and $A(j w)$ is the Fourier transformation of $a(t)$, then:

$$
\int_{-\infty}^{\infty}|a(t)|^{2} d t=\frac{1}{2 \pi} \int_{-\infty}^{\infty}|A(j w)|^{2} d w
$$

where $|A(j w)|^{2}$ is the power spectral density of the acceleration $a(t)$.

The relevant tremor energy becomes

$$
E=\frac{1}{2 \pi} \int_{W_{L}}^{W_{U}}|A(j w)|^{2} d w
$$

$W_{L}$ and $W_{U}$ are the tremor frequency range, which are 4 and $8 \mathrm{~Hz}$, respectively. And $E$ is the energy assigned to the ET relevant frequencies for the specific period of time.

Tremor was classified into four levels corresponding to the FTMS scale according to the energy of the tremor in the frequency domain. The basic analysis unit in this research is $1 \mathrm{~min} ; 1,500$ records were included in each basic analysis unit and around 160 values locate in the $4-8 \mathrm{~Hz}$ frequency range. The data collected in $1 \mathrm{~h}$ from four patients (with tremor grades 1-4, respectively, according to the evaluation of the neurologists) were adopted to define the thresholds.

First, FFT operation was applied and the tremor energy was calculated for each minute (60 values for each patient and 240 values in total) as it is shown in Figure 1A. Second, the 240 energy values were classified into four groups using the K-mean clustering method, and the result is explained in Table 1 and Figure 1B.

Then, the thresholds are defined as below:

$$
\mathrm{Th}_{i}=0.5^{*}\{\operatorname{Max}(\text { Cluster } i)+\operatorname{Min}[(\text { Cluster } i+1)]\}(i=1,2,3)
$$

The results are rounded to integers as the three thresholds, which are 425,904 , and 1,202 $\left(\mathrm{mG}^{2}\right)$, respectively. They classify the tremor into four levels ("Light," "Medium," "High," and "Very high") corresponding to grades 1-4 of FTMTRS, respectively. The FTMTRS scores of the neurological rating obtained during standardized assessments at hospital were used as a gold standard, and the mean FTMTRS scores of all six tasks were employed for correlation analysis (Pearson correlation, $r=0.80$; $p=0.005)$. After this procedure, tremor condition of $1 \mathrm{~min}$ can 

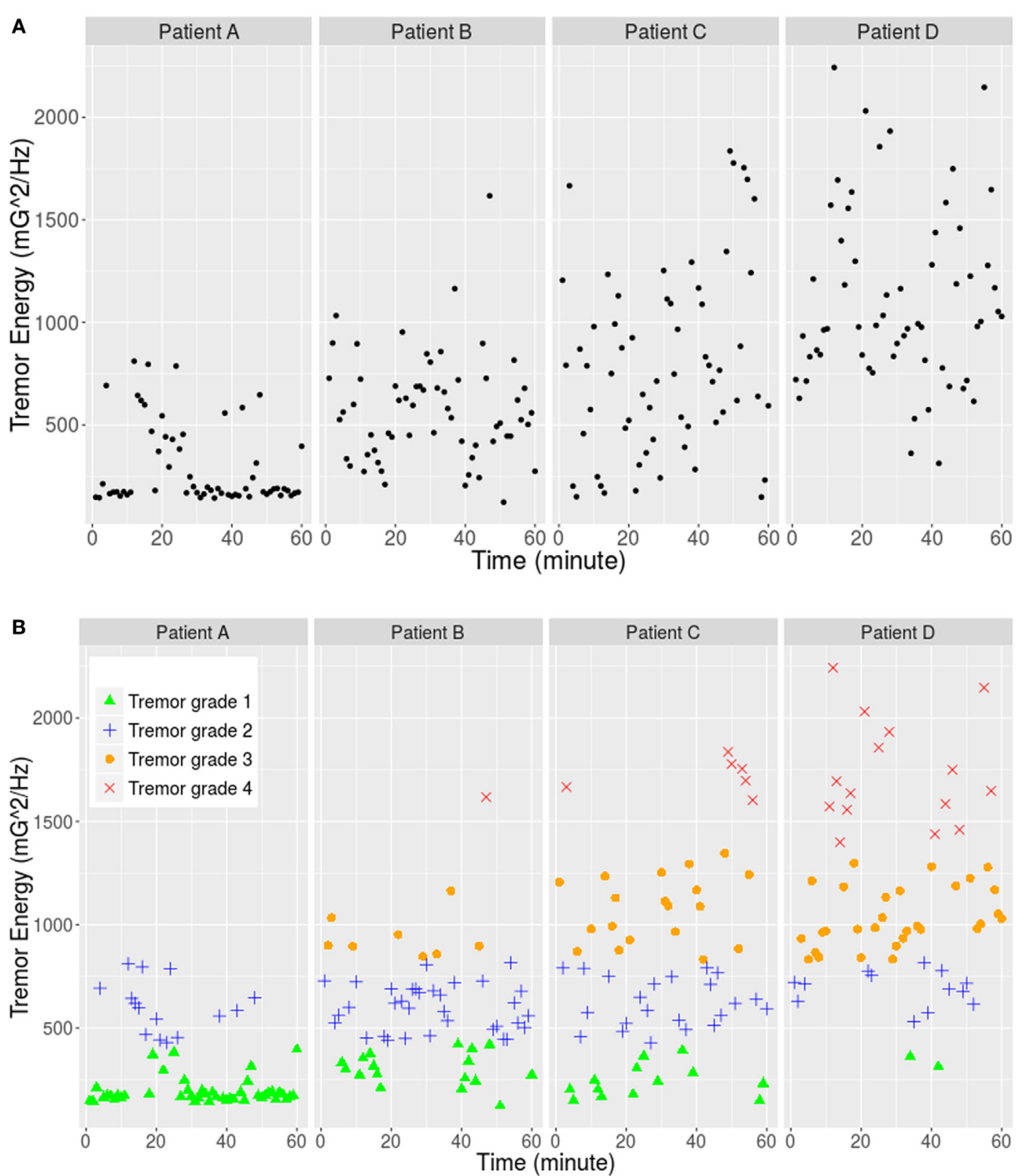

FIGURE 1 | (A) Tremor energy between frequency range 4 and $8 \mathrm{~Hz}$ of each minute for four patients. (B) K-mean clustering result: the data were separated into four classes corresponding to the four tremor grades of FTMS.

TABLE 1 | K-mean clustering result of the tremor energy based on the data collected from four patients.

\begin{tabular}{lcccc}
\hline & Cluster $\mathbf{1}$ & Cluster $\mathbf{2}$ & Cluster 3 & Cluster 4 \\
\hline Cluster size & 77 & 84 & 57 & 22 \\
Cluster means & 229.30 & 620.36 & $1,036.89$ & $1,722.60$ \\
Max value & 421.03 & 816.34 & $1,004.71$ & $2,243.06$ \\
Min value & 124.05 & 429.54 & 993.42 & $1,398.99$
\end{tabular}

be represented with a single quantitative value. By combining the analysis result of every minute, the tremor condition in longer period can be revealed, for instance, in each hour or each day.

\section{Data Generalization}

Since the proposed data collecting system is able to collect the daily movement data of the patients continuously, a larger scale analysis covering a longer period is possible. As the ET energy is not constant in time, it is convenient to provide a time independent yet consistent method to present the ET level. By using this method, it will be possible to compare different episodes for the same patient or different patient results. The proposed method represents a bi-dimensional diagram where on the $Y$ axis represents the maximum energy value, $\max _{i \in[1, ., N]}(E(i))$, where $N$ is the number of minutes under consideration and $E(i)$ is the energy 
associated with every minute. On the $X$ axis, the averaged energy $\sum_{i=1}^{N} E(i) / N$ is represented, in such a way that the structure of this chart remembers the Receiver Operating Characteristic diagram.

TABLE 2 | Summary of the data collected from the eight patients.

\begin{tabular}{lc} 
Item & Value \\
\hline Total number of patients & 8 \\
Total number of hours (days) & 208 (16 days) \\
Hours per patient (minimum) & 17 \\
Hours per patient (maximum) & 34 \\
Hours per patient (mean) & 26 \\
Hours per patient (SD) & 6.05
\end{tabular}

High $Y$ values and low $X$ values or low $Y$ values and high $X$ values mean low ET intensity. Therefore, it is feasible to use the area of the rectangle defined by $(0,0)$ and the point representing the patient data as the ET intensity metrics. Thus, tremor situation of every hour can be represented with a single point, then, the analysis scale can be enlarged by 60 times.

\section{RESULTS}

All patients wore the smartwatch consistently and performed the standardized tasks as required every day. Each patient wore the smartwatch 3 days at least and the data of 2 days from each of them were selected for the analysis. More details

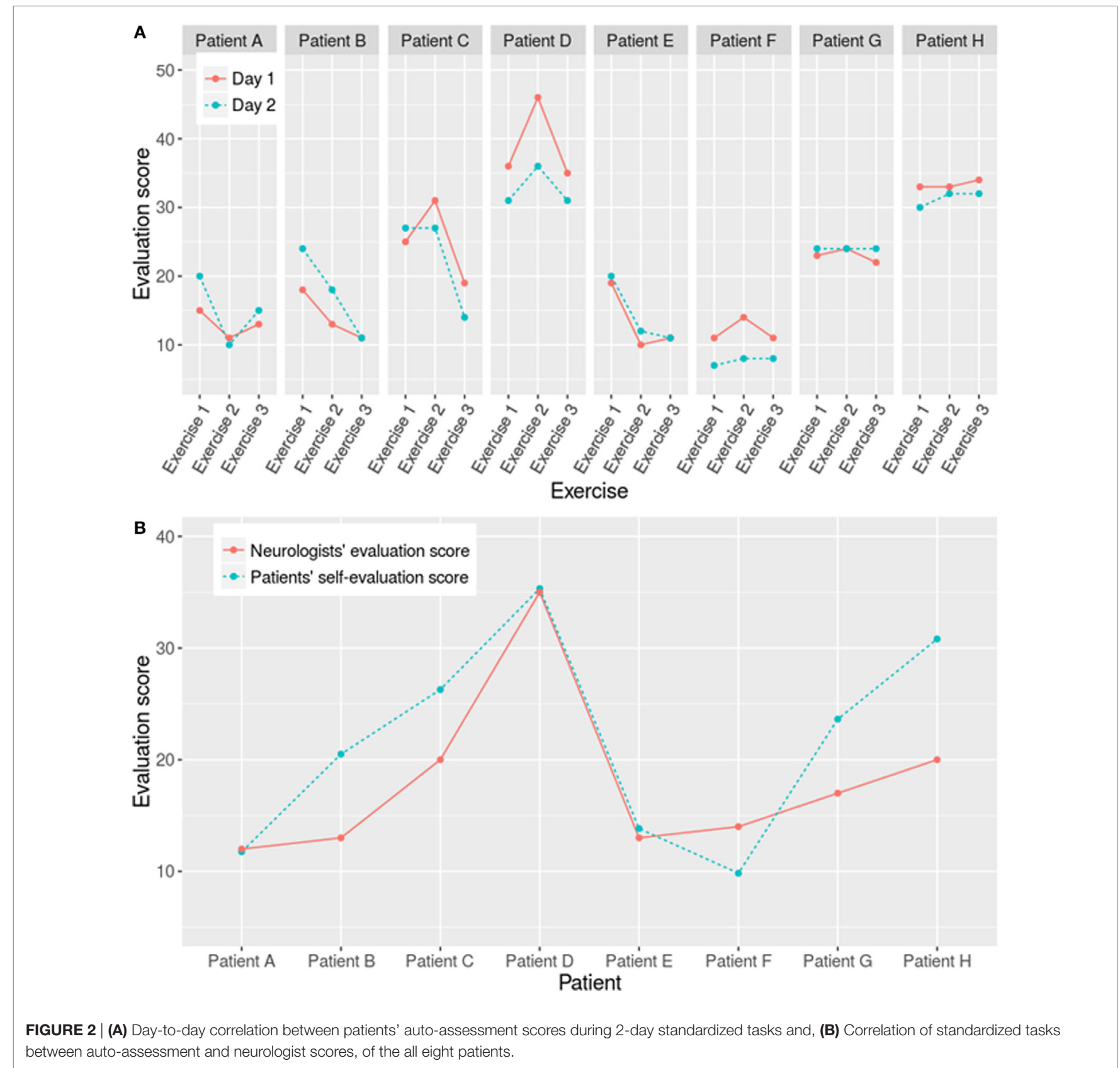


about the data collected from the eight patients are shown in Table 2.

Patient's self-assessment scores between tremor ratings from days 1 and 2 for each patient show good agreement $(r=0.87$, 95\% CI: $0.72-0.94, p<0.001$ ) (Figure 2A). Significant correlation is also obtained between neurologist's FTMTRS and patient's FTMTRS mean auto-assessment scores ( $r=0.84,95 \%$ CI: $0.33-0.97, p=0.009$ ) (Figure 2B), both scores of all patients are listed in Table 3.

TABLE 3 | Rating scores of the patient's self-assessment and neurologists' assessment.

\begin{tabular}{lcc}
\hline Patient no. & $\begin{array}{c}\text { Mean Fahn-Tolosa-Marin } \\
\text { Tremor Rating Scale score } \\
\text { All test, all days; patient's } \\
\text { auto-assessment }\end{array}$ & $\begin{array}{c}\text { Neurologist } \\
\text { score }\end{array}$ \\
\hline A & $23.63( \pm 0.92)$ & 17 \\
B & $35.33( \pm 5.61)$ & 35 \\
C & $20.50( \pm 6.32)$ & 13 \\
D & $9.83( \pm 2.64)$ & 14 \\
E & $13.83( \pm 4.45)$ & 13 \\
F & $26.28( \pm 5.11)$ & 20 \\
G & $11.75( \pm 5.05)$ & 12 \\
H & $30.81( \pm 1.36)$ & 20 \\
\hline
\end{tabular}

TABLE 4 | Basic information of the patients and healthy subject involved in the experiment.

\begin{tabular}{lcccc} 
Subject & Sex & Age & Tremor grade & Arm with higher tremor \\
\hline Healthy subject & Male & 54 & 0 & None \\
Patient A & Male & 68 & 1 & Right \\
Patient B & Male & 72 & 2 & Right \\
Patient C & Male & 75 & 3 & Left \\
Patient D & Male & 60 & 4 & Equal
\end{tabular}

The data of four patients and the healthy subject were further analyzed considering their tremor levels and data integrity (each represents a tremor level). Their information is shown in Table 4. Figure 3 exhibits the validation result using the similar method as Pulliam et al. (28). The correlation between the patients' selfassessment score and the calculation result based on the collected data is significant ( $r=0.97,95 \%$ CI: $0.11-0.99, p=0.032$ ).

Patient's tremor in each minute was classified according to the predefined thresholds. The general situation during long-term daily lives was summarized by combining the classification result of every minute. A graphical representation of patient D's tremor (severe ET) during different time periods is illustrated in Figure 4. This analysis allows not only quantifying the percentage of time in a day with different levels of tremor severity but also querying the moments with more serious tremor (in this case between 18 and $20 \mathrm{~h}$ ), a potential tool to optimize treatment.

Finally, Figure 5 presents the daily $(10 \mathrm{~h})$ tremor ratings of Patient A and Patient D (with the lowest and the highest tremor severity, respectively). The vertical and horizontal components of each point indicate the max and the mean tremor energy value during this hour, respectively. The distribution of patient A's tremor is closer to the bottom left corner with mean energy less than $400(\mathrm{mG})^{2} / \mathrm{Hz}$ and max energy less than 1,500 except $2 \mathrm{~h}$ at noon (12 and 13 o'clock). In contrast, patient D's tremor ratings are closer to the top right corner at the most of the time.

\section{DISCUSSION}

This work evaluates the feasibility of continuously monitoring the tremor status of ET patients by using a highly portable system, which is based on a smartwatch and a smartphone.

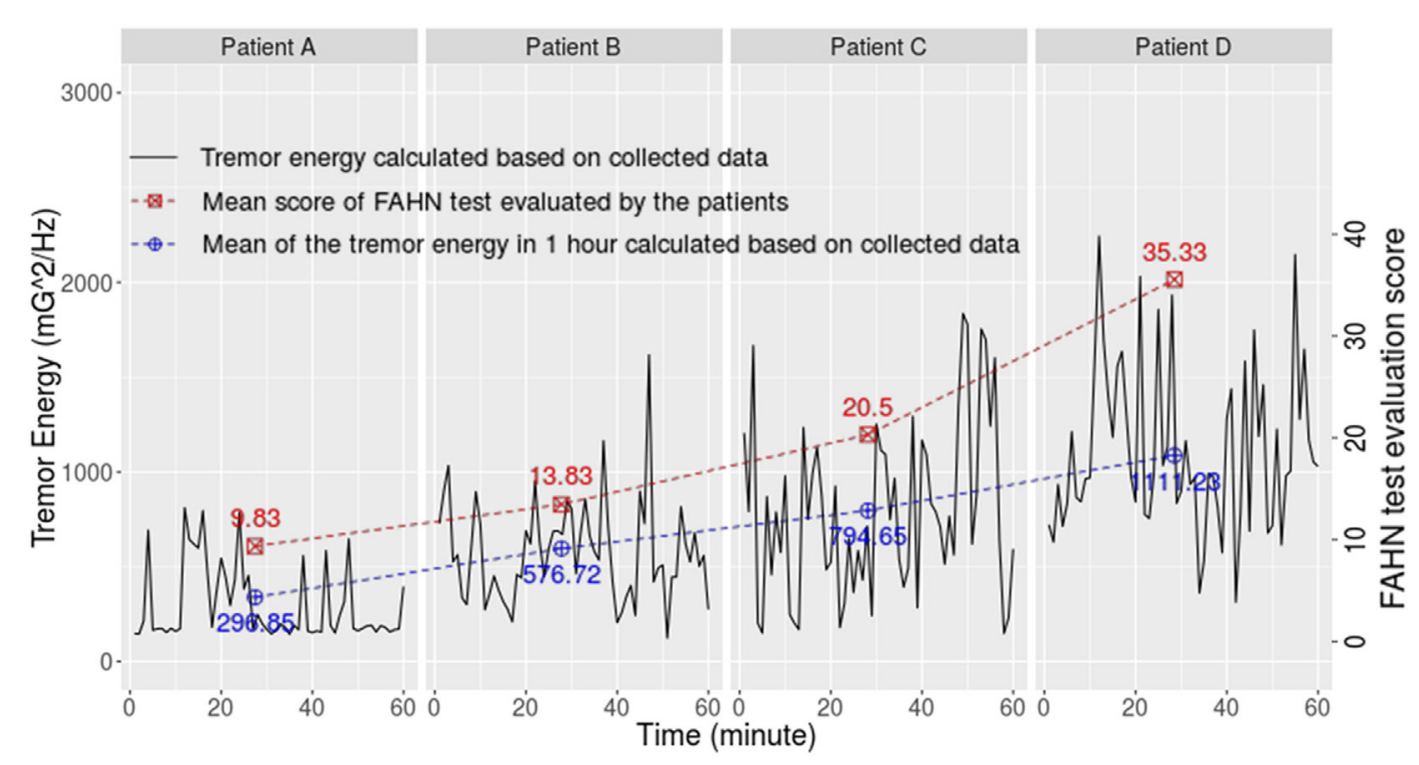

FIGURE 3 | Correlation between results based on collected data and the auto-assessment scores reported by four patients with different tremor degrees. 

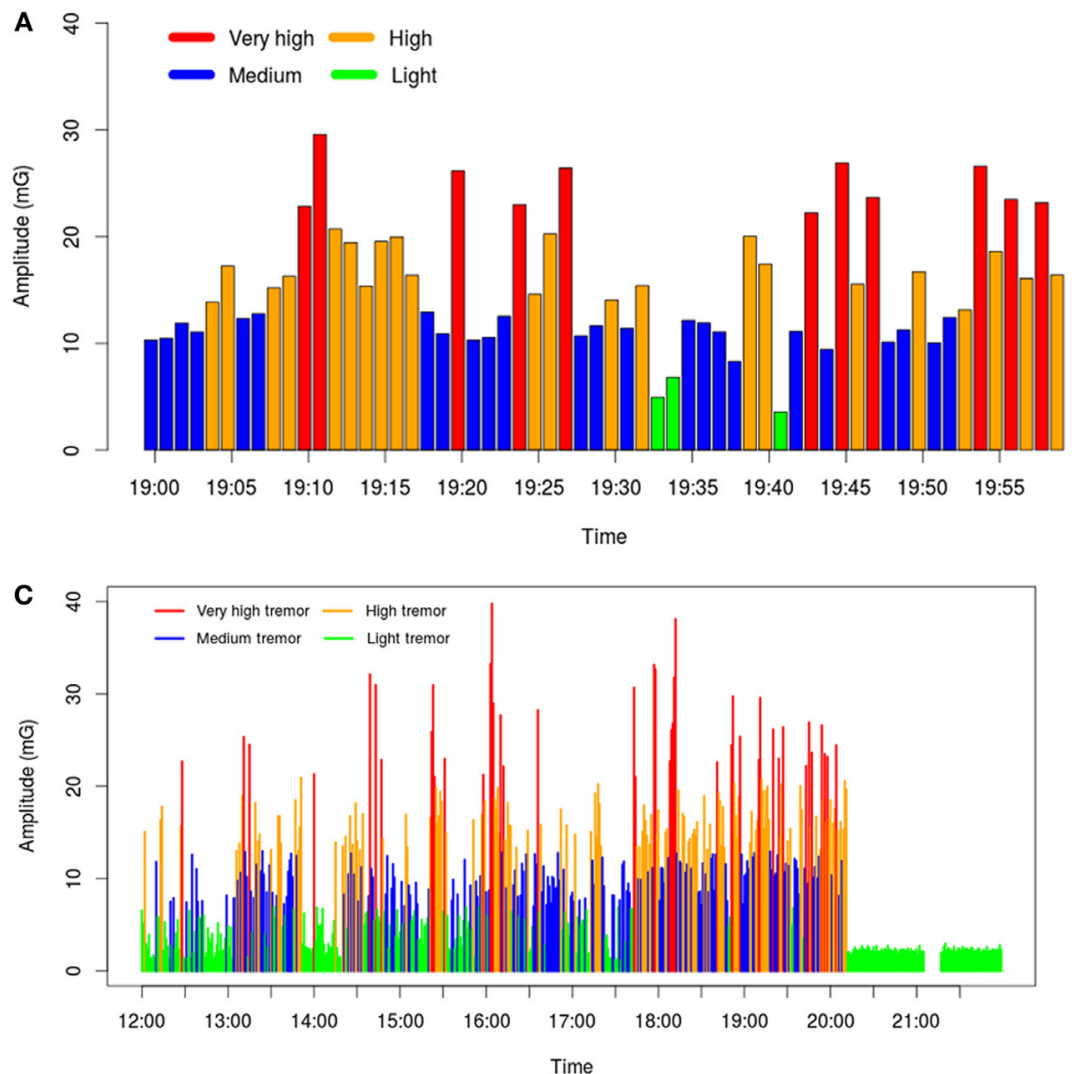

B

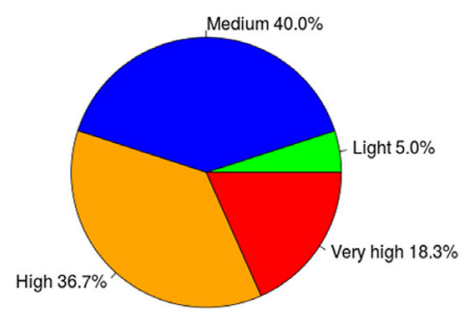

D

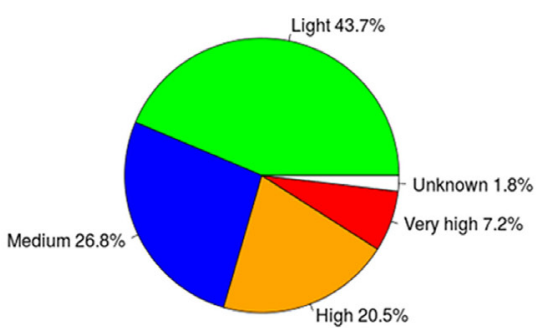

FIGURE 4 | (A) Tremor severities of every minute in an hour (19:00-19:59). (B) Percent of each level of tremor during this hour: high- and very high-level tremor appears in more than $65 \%$ of the overall time. (C,D) illustrate the collected data in a longer range, which better reflects the overall situation of the patient during $8 \mathrm{~h}$ (50\% of the day awake). The "Unknown" part means the data during this period are failed to be recorded.

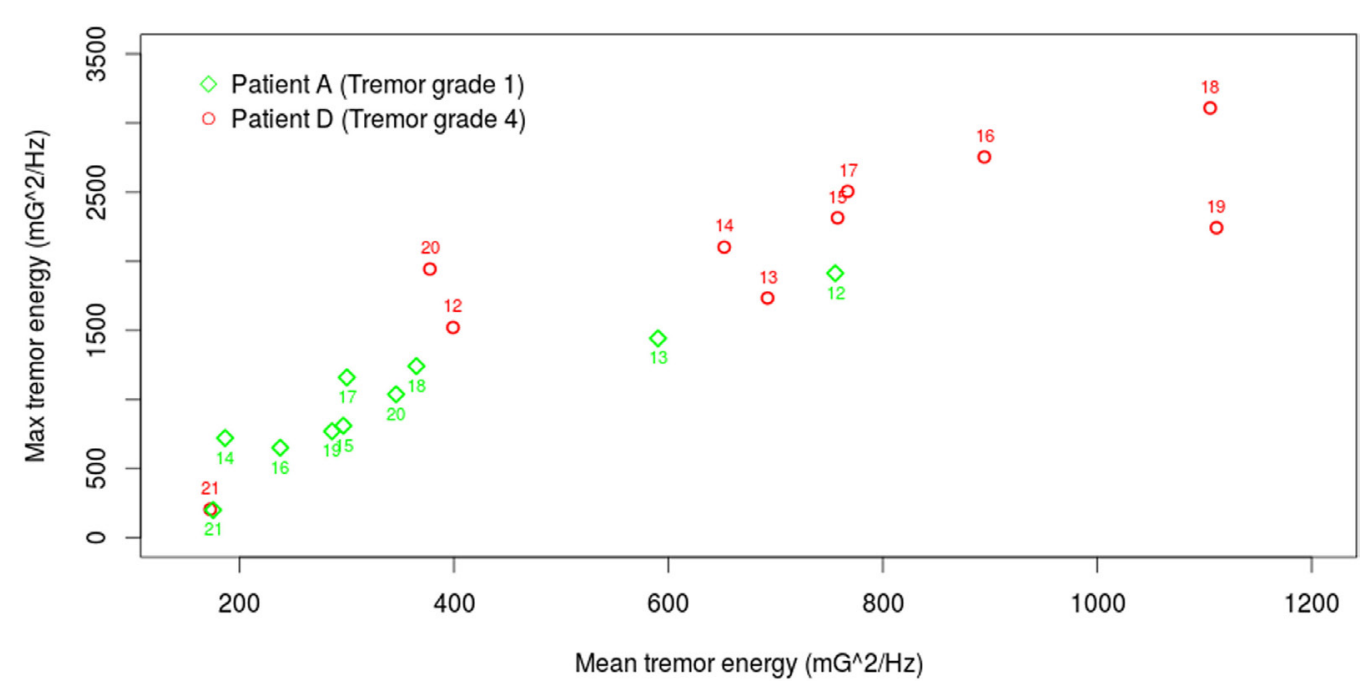

FIGURE 5 | Daily analysis of tremor rating of patients A and D, who have the lowest and the highest tremor severity, respectively.

With the data collected with this system, hypothetically it would be able to trace quantitatively the progression of the disease in the long term, as well as to measure the effect of different treatments more accurately. The major temporal resolution of tremor status obtained could have great potential in clinical practice and research. 
Although we have used for the first time FTMTRS as a gold standard to compare its ratings with the accelerometer's quantitative measures in ET, this scale includes tasks for postural and kinetic tremor which have demonstrated good correlation with data of motor sensors (9). It has also shown good reliability for evaluating upper extremities tremor, and finally, its logarithmic relationship with tremor amplitude, measured with transducers, has been well documented (32). Since the tasks to measure the intensity of tremor are easy to learn, we have contemplated that patients make their standardized auto-assessment of the severity tremor in order to use the results in our analysis. The correlation among all tremor assessments-from the neurologists, the patients, and the system - has been robust with minimum deviation, being this finding a push for starting to consider autoassessment as a valid measure, which could avoid videotaping tremor tasks and subsequent ratings by physicians. In agreement with Pulliam et al. (28), the strong relationship between continuous monitoring and standardized assessment would make possible to dispense with the latest in clinical practice.

\section{System Advantages}

This system is highly portable and comfortable. Patients only need to wear the smartwatch and carry the smartphone with them. This will not introduce extra inconvenience, since both devices are very common daily supplies. The communication between the watch and the smartphone is through Bluetooth, allowing the patient to carry the smartphone anywhere around. The data uploading is supported by both Wi-Fi and 3G/4G telephone services, and it is temporarily stored in the smartphone in case that there is no signal, in which case it is later uploaded once Internet is available. The data are managed by an online database, which is accessible remotely. Clinicians are able to trace the situation of the patients at any time through Internet, no matter where the patients are. Moreover, the cost of the system is very low-the main cost is the smartwatch-and it is specially designed for aged people.

\section{Clinical Application}

Compared with the traditional tremor rating scales, this system provides a more accurate quantitative evaluation of the tremor severity for ET patients. It eliminates the bias produced due to the subjective judgment of the neurologist and the patient. Since there is no distance limitation for this system, it is possible for the neurologists to monitor a patient remotely.

The long-term and unrestricted tremor analysis could be a potentially useful tool to determine the fluctuation patterns of ET caused by voluntary movements and other internal or external factors (alcohol ingestion, stress, etc.), as well as to improve treatment efficacy and effectiveness. Regarding the first point, tremor amplitude fluctuations in ET have been reported around

\section{REFERENCES}

1. Louis ED, Ferreira JJ. How common is the most common adult movement disorder? Update on the worldwide prevalence of essential tremor. Mov Disord (2010) 25:534-41. doi:10.1002/mds.22838

2. Louis ED. Essential tremor: from bedside to bench and back to bedside Curr Opin Neurol (2014) 27:461-7. doi:10.1097/WCO.0000000000000115
$30-50 \%$ between assessments using rating scales (33). On the other hand, evidences with sensor motor monitoring are so far scarce and inconclusive; it was reported a $23 \%$ variability in an objective assessment performed with a motor sensor system (34), in which these fluctuations were statically associated with high amplitude tremor. However, other authors have not found great amplitude variability in ET patients with continuous monitoring (28). Therefore, the objective assessment and continuous monitoring with accelerometers could be to contribute to know better the nature of this tremor. With respect to the optimization of the treatment, given that clinicians can objectively distinguish and quantify percentages of time throughout the day with different grades of tremor severity, it would be possible to monitor more accurately the effects of the treatment applied. Objective and continuous register are necessary to evaluate the treatment interventions in clinical trial.

\section{Limitations}

In this study, the background activities when tremor appears during the patients' daily lives were not analyzed. An applet was installed in the smartphone to help patients to record their activities and the feeling of tremor intensity in that moment, but they forgot to record frequently. Therefore, we did not include these data in this study. Current effort is making to simplify the applet and to introduce some alert to remind patients of this task. Knowing the background activities will permit a better traceability of tremor.

This system collects the movement data of patient's arm. However, tremor of many ET patients also appears in head and legs. In these situations, the data collected with this system cannot accurately reflect the severity of the disease. Further development is necessary to connect more wearable devices into the system.

\section{AUTHOR CONTRIBUTIONS}

YA and JO-M conceptualized the idea for this study and organized the project. XZ, AVC, JO-M, and YA were involved in the data collection. XZ and JO-M were responsible for the data analysis, and the analysis results were reviewed and critiqued by YA, JO-M, and JB. The patients were managed and evaluated by AVC, YA, JB, and SLM. The final manuscript was written by XZ and YA and was reviewed and improved by JO-M and JB.

\section{ACKNOWLEDGMENTS}

The authors express their recognition to the Investigation Foundation of University Hospital of Getafe, Madrid and to the "Universidad Politécnica de Madrid" for the support they have provided. They also recognize the support provided by the Chinese Scholarship Council (CSC).

3. Matsumoto Y, Seki M, Ando T, Kobayashi Y, Iijima H, Nagaoka M, et al. Tremor frequency based filter to extract voluntary movement of patients with essential tremor. 2012 4th IEEE RAS \& EMBS International Conference on Biomedical Robotics and Biomechatronics (BioRob). Rome: IEEE (2012).

4. Elias WJ, Shah BB. Tremor. JAMA (2014) 311:948-54. doi:10.1001/ jama.2014.6231 
5. Fasano A, Deuschl G. Therapeutic advances in tremor. Mov Disord (2015) 30:1557-65. doi:10.1002/mds.26383

6. Heldman DA, Jankovic J, Vaillancourt DE, Prodoehl J, Elble RJ, Giuffrida JP. Essential tremor quantification during activities of daily living. Parkinsonism Relat Disord (2011) 17:537-42. doi:10.1016/j.parkreldis.2011. 04.017

7. Jankovic J. Parkinson's disease: clinical features and diagnosis. J Neurol Neurosurg Psychiatry (2008) 79:368-76. doi:10.1136/jnnp.2007.131045

8. Budzianowska A, Honczarenko K. Assessment of rest tremor in Parkinson's disease. Neurol Neurochir Pol (2008) 42:12-21.

9. Mostile G, Giuffrida JP, Adam OR, Davidson A, Jankovic J. Correlation between Kinesia system assessments and clinical tremor scores in patients with essential tremor. Mov Disord (2010) 25:1938-43. doi:10.1002/mds. 23201

10. Louis ED, Wendt KJ, Albert SM, Pullman SL, Yu Q, Andrews H. Validity of a performance-based test of function in essential tremor. Arch Neurol (1999) 56:841-6. doi:10.1001/archneur.56.7.841

11. Louis ED, Barnes L, Wendt KJ, Ford B, Sangiorgio M, Tabbal S, et al. A teaching videotape for the assessment of essential tremor. Mov Disord (2001) 16:89-93. doi:10.1002/1531-8257(200101)16:1<89::AID-MDS1001> 3.0.CO;2-L

12. Fahn F, Tolosa E, Marin C. Clinical rating scale for tremor. Parkinsons Dis Mov Disord (1993) 2:271-80. doi:10.7916/D8WM1C41

13. Rigas G, Tzallas AT, Tsipouras MG, Bougia P, Tripoliti EE, Baga D, et al. Assessment of tremor activity in the Parkinson's disease using a set of wearable sensors. IEEE Trans Inf Technol Biomed (2012) 16:478-87. doi:10.1109/ TITB.2011.2182616

14. Findley LJ. Classification of tremors. J Clin Neurophysiol (1996) 13:122-32. doi:10.1097/00004691-199603000-00003

15. Deuschl G, Krack P, Lauk M, Timmer J. Clinical neurophysiology of tremor. J Clin Neurophysiol (1996) 13:110-21. doi:10.1097/00004691-19960 3000-00002

16. Elble RJ. Essential tremor frequency decreases with time. Neurology (2000) 55:1547-51. doi:10.1212/WNL.55.10.1547

17. Thanvi B, Lo N, Robinson T. Essential tremor-the most common movemen disorder in older people. Age Ageing (2006) 35(4):344-9. doi:10.1093/ageing/ afj072

18. Ahlrichs $\mathrm{C}$, Samà $\mathrm{A}$. Is frequency distribution enough to detect tremor in PD patients using a wrist worn accelerometer? Proceedings of the 8th International Conference on Pervasive Computing Technologies for Healthcare. Oldenburg: ICST (Institute for Computer Sciences, Social-Informatics and Telecommunications Engineering) (2014).

19. Patel S, Hughes R, Huggins N, Standaert D, Growdon J, Dy J, et al. Using wearable sensors to predict the severity of symptoms and motor complications in late stage Parkinson's disease. 30th Annual International Conference of the IEEE Engineering in Medicine and Biology Society, 2008. EMBS 2008. Vancouver: IEEE (2008).

20. Patel S, Lorincz K, Hughes R, Huggins N, Growdon J, Standaert D, et al. Monitoring motor fluctuations in patients with Parkinson's disease using wearable sensors. IEEE Trans Inf Technol Biomed (2009) 13:864-73. doi:10.1109/TITB.2009.2033471

21. Smeja M, Foerster F, Fuchs G, Emmans D, Hornig A, Fahrenberg J. 24-h assessment of tremor activity and posture in Parkinson's disease by multi-channel accelerometry. J Psychophysiol (1999) 13:245. doi:10.1027//0269-8803.13.4.245
22. Hoff JI, Wagemans EA, van Hilten BJ. Ambulatory objective assessment of tremor in Parkinson's disease. Clin Neuropharmacol (2001) 24:280-3. doi:10.1097/00002826-200109000-00004

23. Mamorita N, Iizuka T, Takeuchi A, Shirataka M, Ikeda N. Development of a system for measurement and analysis of tremor using a three-axis accelerometer. Methods Inf Med (2009) 48:589-94. doi:10.3414/ME9243

24. Zwartjes DG, Heida T, Van Vugt JP, Geelen JA, Veltink PH. Ambulatory monitoring of activities and motor symptoms in Parkinson's disease. IEEE Trans Biomed Eng (2010) 57:2778-86. doi:10.1109/TBME.2010. 2049573

25. Joundi RA, Brittain JS, Jenkinson N, Green AL, Aziz T. Rapid tremor frequency assessment with the iPhone accelerometer. Parkinsonism Relat Disord (2011) 17:288-90. doi:10.1016/j.parkreldis.2011.01.001

26. Thanawattano C, Anan C, Pongthornseri R, Dumnin S, Bhidayasiri R. Temporal fluctuation analysis of tremor signal in Parkinson's disease and essential tremor subjects. Conf Proc IEEE Eng Med Biol Soc (2015) 2015:6054-7. doi:10.1109/EMBC.2015.7319772

27. Daneault JF, Carignan B, Codère CE, Sadikot AF, Duval C. Using a smart phone as a standalone platform for detection and monitoring of pathological tremors. Front Hum Neurosci (2013) 6:357. doi:10.3389/fnhum. 2012.00357

28. Pulliam SL, Eichenseer SR, Goetz CG, Waln O, Hunter CB, Jankovic J, et al. Continuous in-home monitoring of essential tremor. Parkinsonism Relat Disord (2014) 20:37-40. doi:10.1016/j.parkreldis.2013.09.009

29. Wile DJ, Ranawaya R, Kiss ZH. Smart watch accelerometry for analysis and diagnosis of tremor. J Neurosci Methods (2014) 230:1-4. doi:10.1016/j. jneumeth.2014.04.021

30. Zheng X, Ordieres-Mere J. Development of a human movement monitoring system based on wearable devices. The International Conference on Electronics, Signal Processing and Communication Systems (ESPCO 2014). Athens (2014).

31. Zheng X, Ordieres-Mere J. Detection and analysis of tremor using a system based on smart device and NoSQL database. 2015 International Conference on Industrial Engineering and Systems Management (IESM). Seville: IEEE (2015).

32. Elble RJ, Pullman SL, Matsumoto JY, Raethjen J, Deuschl G, Tintner R, et al. Tremor amplitude is logarithmically related to 4- and 5-point tremor rating scales. Brain (2006) 29:2660-6. doi:10.1093/brain/awl190

33. Elble RJ, Higgins C, Leffler K, Hughes L. Factors influencing the amplitude and frequency of essential tremor. Mov Disord (1994) 9:589-96. doi:10.1002/ mds.870090602

34. Mostile G, Fekete R, Giuffrida JP, Yaltho T, Davidson A, Nicoletti A, et al. Amplitude fluctuations in essential tremor. Parkinsonism Relat Disord (2012) 18:859-63. doi:10.1016/j.parkreldis.2012.04.019

Conflict of Interest Statement: The authors declare that the research was conducted in the absence of any commercial or financial relationships that could be construed as a potential conflict of interest.

Copyright (c) 2017 Zheng, Vieira Campos, Ordieres-Meré, Balseiro, Labrador Marcos and Aladro. This is an open-access article distributed under the terms of the Creative Commons Attribution License (CC BY). The use, distribution or reproduction in other forums is permitted, provided the original author(s) or licensor are credited and that the original publication in this journal is cited, in accordance with accepted academic practice. No use, distribution or reproduction is permitted which does not comply with these terms. 


\subsection{Publication 3}

- Title: Comparison of Data Preprocessing Approaches for Applying Deep Learning to Human Activity Recognition in the Context of Industry 4.0

- Authors: Xiaochen Zheng, Meiqing Wang, Joaquín Ordieres-Meré

- Publication date: 2018/7/3

- Journal: Sensors

Impact Factor: 2.475 (2017) / 3.014 (5 year)

Quartile: Q2

ISSN: $1424-8220$

Publisher: Multidisciplinary Digital Publishing Institute, BASEL, SWITZERLAND

- Volume: 18

- Issue: 7

- Pages: 2146

- DOI: https://doi.org/10.3390/s18072146

- Copyright statement: This article is published in Open Access journals. According to the publisher's policy, "Articles published in Sensors will be Open-Access articles distributed under the terms and conditions of the Creative Commons Attribution License (CC BY). The copyright is retained by the author(s).' 3

\footnotetext{
${ }^{3}$ https://www.mdpi.com/journal/sensors/about
} 
Article

\title{
Comparison of Data Preprocessing Approaches for Applying Deep Learning to Human Activity Recognition in the Context of Industry 4.0
}

\author{
Xiaochen Zheng ${ }^{1}$, Meiqing Wang ${ }^{2}$ and Joaquín Ordieres-Meré ${ }^{1, *(\mathbb{D})}$ \\ 1 Department of Industrial Engineering, ETSII, Universidad Politécnica de Madrid, 28006 Madrid, Spain; \\ xiaochen.zheng@alumnos.upm.es \\ 2 School of Mechanical Engineering and Automation, Beihang University (BUAA), Beijing 100083, China; \\ sy1514206@buaa.edu.cn \\ * Correspondence: j.ordieres@upm.es; Tel.: +34-913-36-3144
}

Received: 24 May 2018; Accepted: 29 June 2018; Published: 3 July 2018

\begin{abstract}
According to the Industry 4.0 paradigm, all objects in a factory, including people, are equipped with communication capabilities and integrated into cyber-physical systems (CPS). Human activity recognition (HAR) based on wearable sensors provides a method to connect people to CPS. Deep learning has shown surpassing performance in HAR. Data preprocessing is an important part of deep learning projects and takes up a large part of the whole analytical pipeline. Data segmentation and data transformation are two critical steps of data preprocessing. This study analyzes the impact of segmentation methods on deep learning model performance, and compares four data transformation approaches. An experiment with HAR based on acceleration data from multiple wearable devices was conducted. The multichannel method, which treats the data for the three axes as three overlapped color channels, produced the best performance. The highest overall recognition accuracy achieved was $97.20 \%$ for eight daily activities, based on the data from seven wearable sensors, which outperformed most of the other machine learning techniques. Moreover, the multichannel approach was applied to three public datasets and produced satisfying results for multi-source acceleration data. The proposed method can help better analyze workers' activities and help to integrate people into CPS.
\end{abstract}

Keywords: deep learning; data preprocessing; Human Activity Recognition (HAR); Internet of things (IoT); Industry 4.0

\section{Introduction}

Recent advances in manufacturing industry and Internet of Things (IoT) technology have paved the way for a systematical deployment of cyber-physical systems (CPS), making networked machines perform more efficiently, collaboratively, and resiliently, and transforming manufacturing industries to the Industry 4.0 era [1,2]. According to the Industry 4.0 paradigm, all objects of the factory world are equipped with integrated processing and communication capabilities. This facilitates the vision of the "smart factory", which enables centralized decision-making while requiring distributed manufacturing equipment and resources [3,4]. More "things", even people, need to be connected to the system [5]. In contrast to computer-integrated manufacturing (CIM), the Industry 4.0 movement is not gravitating towards workerless production facilities. Instead, people should be integrated into the cyber-physical structure in such a way that their individual skills and talents can be fully realized [6,7].

The development of IoT technology has also promoted the improvement of Human Activity Recognition (HAR), which is based on copious sensors. HAR has been widely applied in surveillance- 
based security, context-aware computing, ambient assistive living, and assembly tasks analysis [8-14]. A variety of machine learning algorithms have been used to process human activity data in the big data environment [15-20]. In a recently reported study [8], the performance of several common classification methods were compared for recognizing eight daily activities, using the acceleration data collected from wearable sensors in seven different body positions. An overall accuracy of $89 \%$ was achieved using the random forest (RF) method, which outperformed artificial neural network $(\mathrm{ANN})$, decision tree (DT), k-nearest neighbors (k-NN), naive Bayes (NB), and support vector machine (SVM) methods.

Deep learning is a paradigm of machine learning that enables computational models consisting of multiple processing layers to learn representations of data with multiple levels of abstraction [21]. Many studies have proven that the use of deep learning can improve the performance of many applications, especially speech and visual object recognition, in addition to many other domains [21,22]. As a powerful feature extraction mechanism, deep learning has also been used to perform HAR in recent years, and significant improvement has been achieved [23,24]. The convolutional neural network $(\mathrm{CNN})$ is one of the most important deep learning approaches that has been used to perform HAR, and has produced satisfying results in a number of studies [25].

Data preprocessing plays an important role in machine learning and deep learning algorithms, and proper preprocessing of data is compulsory for achieving better HAR performance [26,27]. Kotsiantis et al. [26] defined data preprocessing as including data cleaning, normalization, transformation, feature extraction, and selection. Some of the most well-known algorithms for each step of data preprocessing are presented in their study. More specifically, when performing HAR tasks using inertial data from wearable devices, a segmentation operation is necessary, because raw inertial data fluctuate greatly over time. The segmented data should be transformed into proper formats as the inputs of the deep learning models. Spectrograms are a commonly used data preprocessing method for acceleration data. A spectrogram of an inertial signal is a new representation of the signal as a function of frequency and time. Previous studies [23] have shown that spectrogram representation is essential for extracting interpretable features that represent the intensity differences among nearest inertial data points. A method that combines shallow features and those obtained from deep learning models, in order to overcome the defects that resource limitations cause and the simple design of the deep learning models, was proposed in [23]. However, during our experiment it was found that the spectrogram representation of the acceleration signal does not always produce better classification results, and introducing shallow features does not always improve the overall performance, especially when the dataset is sufficiently large and contains multi-source sensor data.

The aim of this study is to compare different data preprocessing approaches for deep leaning supported HAR tasks in different scenarios, like single or multiple sensors, and provide references for future studies. In this paper, a deep learning algorithm was used to classify daily human activities on the basis of the acceleration data that has been provided by wearable devices in different body positions. The study focused on two important steps-data segmentation and data transformation-of preprocessing acceleration data for deep learning algorithms. A comparison among five data segmentation options was undertaken and the impact of segment length on activity recognition accuracy was analyzed. Four different data transformation methods were compared, including raw acceleration data, the multichannel method, the spectrogram method, and the spectrogram integrated with shallow features method. The highest overall recognition accuracy achieved in this study was $99.42 \%$ for eight daily activities, based on the data from seven wearable sensors, which outperformed most traditional machine learning techniques. Beside the above-mentioned dataset, the chosen multichannel method was also applied to three public HAR datasets, and the results were compared against existing studies. 


\section{Materials and Methods}

The framework of the study is illustrated in Figure 1. The proposed method includes data segmentation, data transformation, deep learning model training, and testing. Human activities are time-dependent, and the raw acceleration data from wearable sensors fluctuates greatly over time, making classification impossible when using a single data point [28]. Most HAR methods are based on a series of data collected in a certain time interval. A segmentation operation is necessary before applying any classification method [8]. The data segments are then transformed into images with four different methods, in order to produce the inputs for the deep learning module. Each input corresponds to a specific deep CNN model and generates a specific classifier. The preprocessed data samples are separated into training and testing samples before the training process. The testing samples are selected randomly. Their quantity depends on the segment options and the total number of samples. More details of each step of the workflow are provided in the following sections.

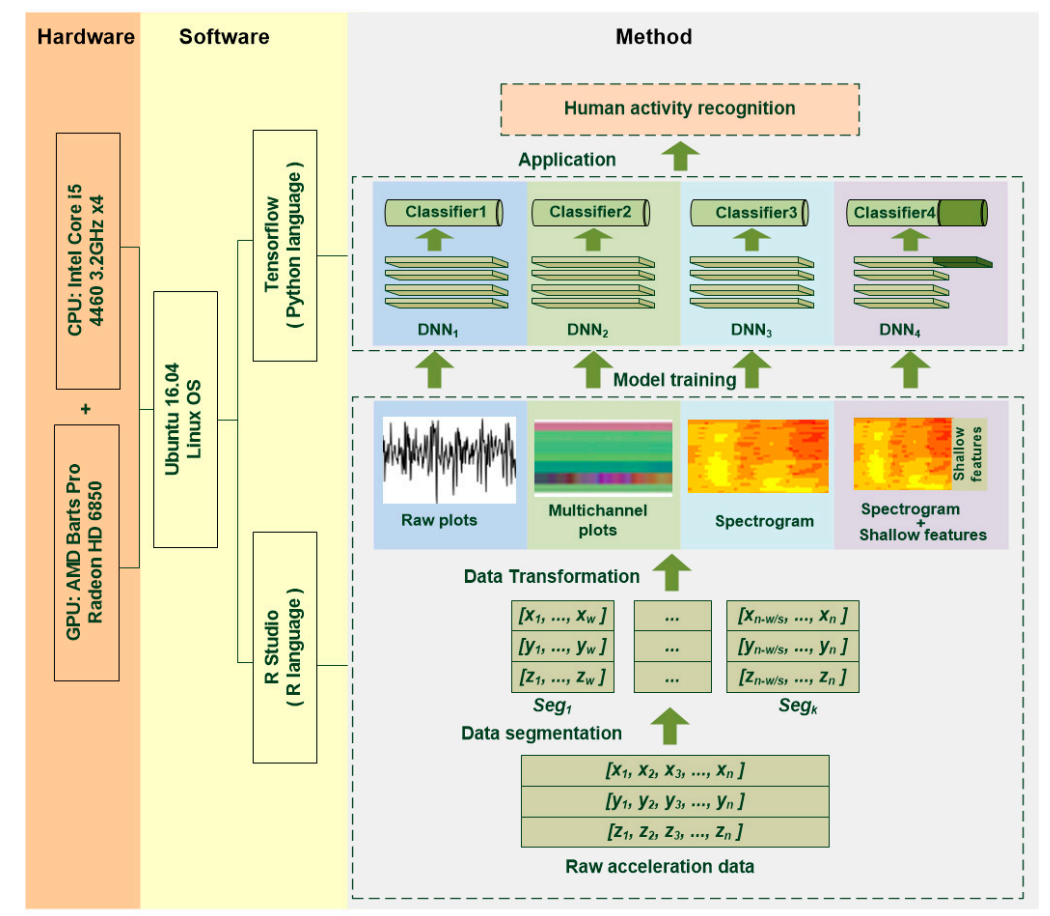

Figure 1. Framework of the proposed method.

\subsection{Data Segmentation}

The raw time-dependent acceleration dataset is separated into segments during the data segmentation process. All of the following HAR-related operations, including feature extraction, classification and validation, etc., are based on these segments. The length of the segments depends on the application context and sampling rate of the sensors. Increasing the length of the segments can improve recognition accuracy, but the training time will be increased and more time will be required to obtain sufficient data. This will cause a delay in response for real-time applications [23] and restrict the application scenarios. In most of the existing studies, segments of 1 to $10 \mathrm{~s}$ are considered for HAR [29].

\subsection{Data Transformation}

In order to generate the proper inputs for the deep learning models, four different data transformation methods were adopted in this study. These methods transform the raw data segments into different type of representations, from which the deep learning models can extract features automatically. The four methods are explained in detail below. 


\subsubsection{Raw Plot}

The raw plot method transforms the acceleration data directly to time series images. The three axes are grouped by column, and the data collected from different positions are grouped by row on the same image, if applicable. Both the $x$-axis and $y$-axis resolution of the produced image are the same as the length of the segment, and the color is black and white. For example, Figure 2 shows the image that is generated from an acceleration data segment, which contains 21 separate sub-images that correspond to three axes (by column) and seven sensors (by row). In this plot, the length of the segments represents the number of values included in this segment. The image resolution $(512 \times 512$ pixels $)$ is not related to the lengths $(512 \times 3)$ of segments. Higher resolutions may produce better results, but the training time will also increase. This method can represent the temporal acceleration variance. The deep learning models are able to extract activity features based on the intensity and shape of the plot at different locations and on different levels.

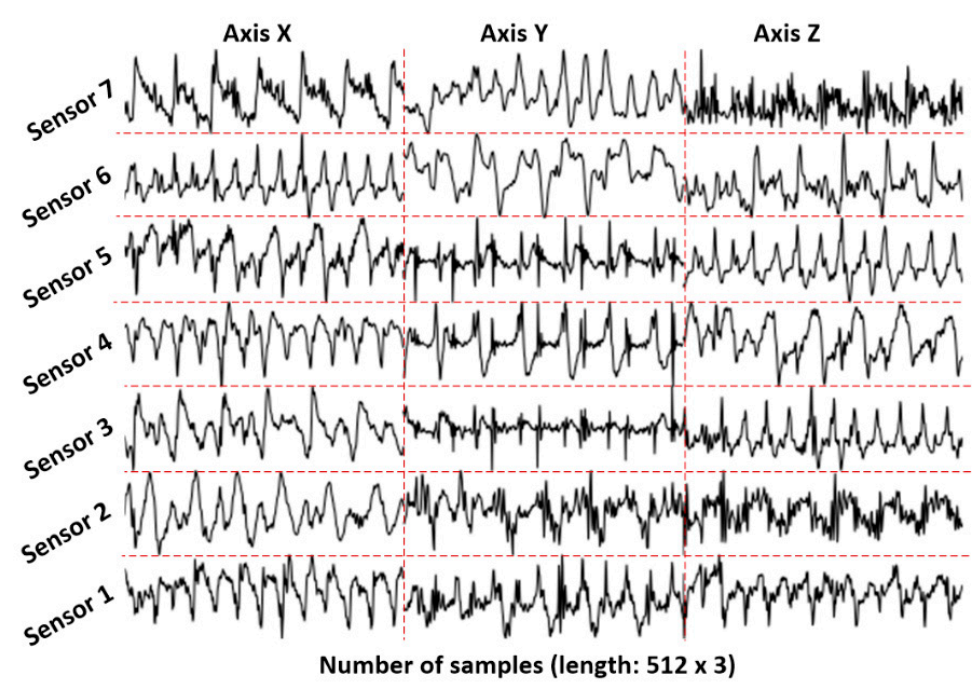

Figure 2. Raw acceleration plot of time domain: segment length 512 and sampling rate $50 \mathrm{~Hz}$ (the dotted lines are added manually for better clarification; image resolution is $512 \times 512$ pixels).

\subsubsection{Multichannel Method}

Unlike the raw plot method, the multichannel method treats the data for the three axes as three overlapped color channels that correspond to red, green, and blue components in the RGB color format. The amplitude of the acceleration signal, which is in the range $(-20,20)$, is projected to a corresponding color value, which is in the range $(0,1)$. In this case, the temporal variance of the acceleration data is transformed into color variance. The three acceleration values of each point are represented as one pixel in the image. The $x$-axis resolution of the image is the same as the length of the segment, and the $y$-axis resolution is the number of sensors. The data collected from different sensors are grouped by row. The advantage of this method is that it reduces the image size enormously and results in a much less training time than the raw plot method. Figure 3 illustrates the principle of this method and an example image produced with this method. The data segment used in this figure is the same as the one used in Figure 2.

\subsubsection{Spectrogram}

The spectrogram of an inertial signal represents the frequency features of the signal in the time domain. It is the magnitude squared of the short-time Fourier transform (STFT). STFT is used to determine the sinusoidal frequency and phase content of local sections of a signal that changes over time $[23,30]$. The procedure for computing the spectrogram is to divide a longer time signal into short windows of equal lengths, and then compute the Fourier transform separately for each shorter 
window. The study by Ravì et al. [23] proved that the spectrogram representation is essential for extracting interpretable features to capture the intensity differences between the nearest inertial data points. The spectrogram representation also provides a form of time and sampling rate invariance. This enables the classification to be more robust against data shifting in time and against changes in the amplitude of the signal and sampling rate.

Figure 4 shows the spectrogram generated from the same data segment that is used in Figures 2 and 3 . Here, the resolution of $y$-axis is 350 pixels and the resolution of $x$-axis is determined by the segment length (L), STFT window length (W), and overlap length (P) by the following equation:

$$
\operatorname{Res}_{\mathrm{x}}=3 \times(\mathrm{L}-\mathrm{W}) /(\mathrm{W}-\mathrm{P}),
$$

The spectrograms of different axes and sensors exhibit different patterns. There is also a difference between different activities.

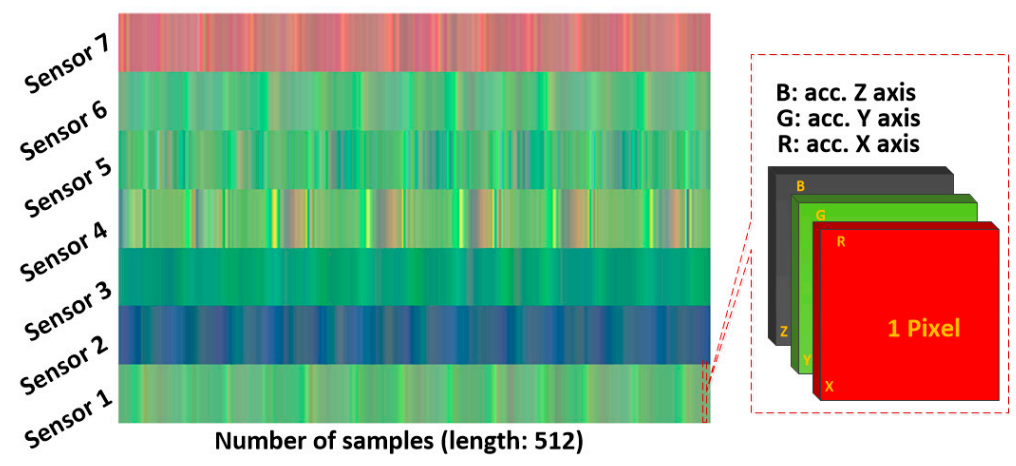

Figure 3. Multichannel RGB color plot on time domain. The segment length is 512 and the sampling rate is $50 \mathrm{~Hz}$ (image resolution $512 \times 7$ pixels).

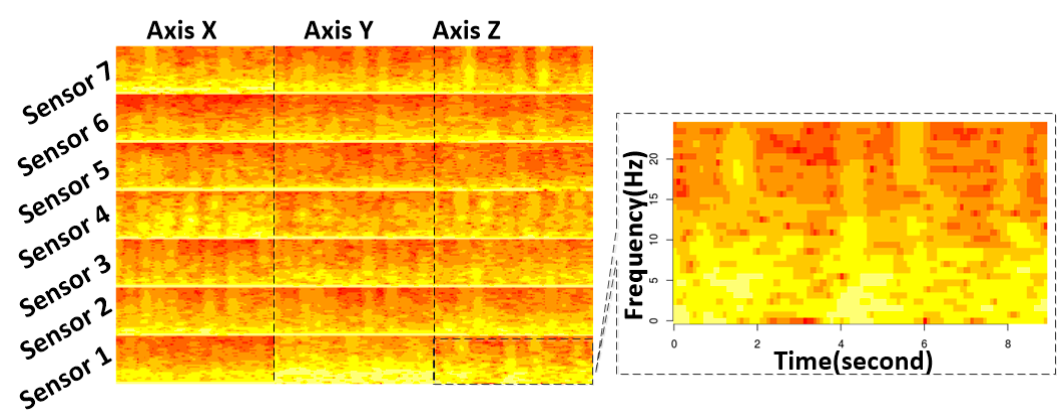

Figure 4. Spectrogram plot of the acceleration data. The segment length is 512 and the sampling rate is $50 \mathrm{~Hz}$, with a short-time Fourier transform (STFT) window length of 64 STFT and an overlap length of 60 STFT (image resolution $336 \times 350$ pixels).

\subsubsection{Spectrogram Combined with Shallow Features}

Previous studies [23] have shown that when data resources are limited, the features that are derived from a deep learning method are sometimes less discriminating than a complete set of predefined shallow features. To overcome this problem, a method of combining both shallow and deep-learned features was proposed in [23], in order to provide complementary information for the classification. This method was also used in this paper, to compare the results against those of the other three methods. The aim is to determine if this method outperforms other methods for multi-source acceleration data. As suggested in [23], 15 shallow features are extracted from the raw acceleration data of each axis and each sensor, as shown in Table 1. These shallow features are combined with the deep-learned features to form the last layer of the deep CNN model. 
Table 1. Shallow features extracted from acceleration data.

\begin{tabular}{cc}
\hline Data & Features \\
\hline Raw signal & $\begin{array}{c}\text { max, min, mean, median, variance, kurtosis, skewness, zero-cross, } \\
\text { root mean square, standard deviation, interquartile range } \\
\text { mean, variance, root mean square, standard deviation }\end{array}$ \\
\hline
\end{tabular}

\subsection{Deep Learning Method}

After preprocessing, the original acceleration data segments are transformed into different types of images, to which the deep learning methods are applied. In this study, the deep CNN algorithm [21-31] is used. Figure 5 shows the overall workflow of the proposed deep CNN method. Different models were built that correspond to the outputs of the four data transformation methods. Each model has its own parameters, such as the number of convolutional layers, the learning rate, pooling size, etc. Following the approach in [23], the shallow features are merged with the deep-learned features on the last fully connected layer, as shown in Figure 5. More details of the deep learning models are available online [32].

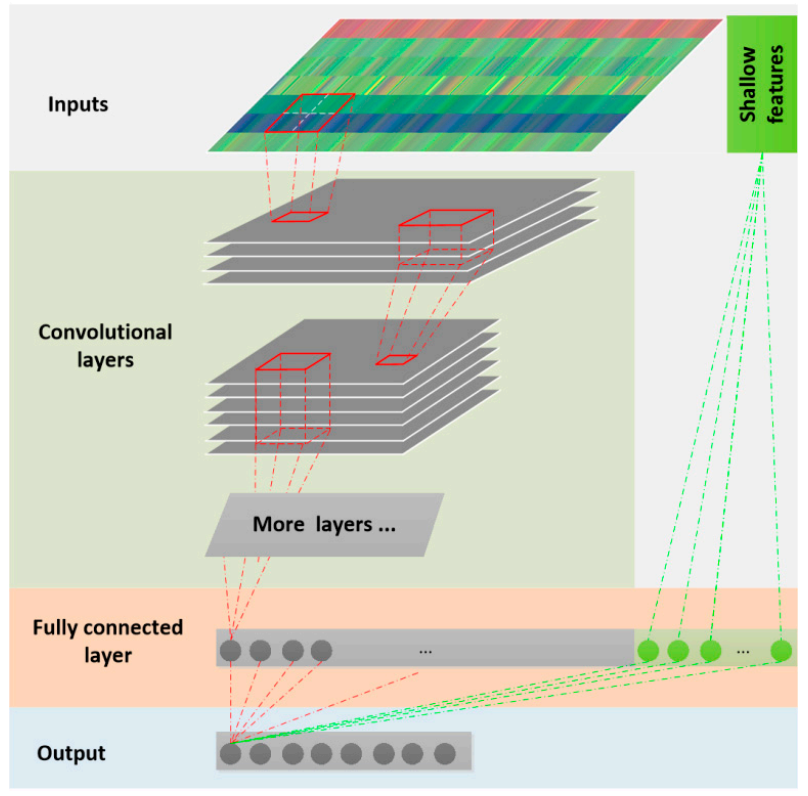

Figure 5. Workflow of deep convolutional neural network (CNN) models.

\section{Results}

\subsection{Dataset and Experimental Setup}

The dataset contributed by Sztyler et al. [8] was adopted to test the proposed methods. The reasons for this were that it is up-to-date and, according to the authors, is the most complete, realistic, and transparent dataset for on-body position detection that is currently available [8]. This dataset contains the acceleration data of eight activities—climbing stairs down (A1), climbing stairs up (A2), jumping (A3), lying (A4), jogging (A5), standing (A6), sitting (A7), and walking (A8)—of 15 subjects (age $31.9 \pm 12.4$, height $173.1 \pm 6.9$, and weight $74.1 \pm 13.8$, with eight males and seven females). For each activity, the acceleration of seven body positions-chest (P1), forearm (P2), head (P3), shin (P4), thigh (P5), upper arm (P6), and waist (P7) -were recorded simultaneously. The subjects performed each activity for roughly $10 \mathrm{~min}$, except for jumping (about $1.7 \mathrm{~min}$ ) due to the physical exertion. In total, the dataset covers $1065 \mathrm{~min}$ of acceleration data for each position and axes, with a sampling 
rate of $50 \mathrm{~Hz}$. We filtered and reorganized the dataset to make it suitable for training deep learning models. The detailed processing method and the prepared datasets are available online [32].

As shown in Figure 1, the experiments in this study were implemented with a computer equipped with a four-core Intel Core i5-4460 3.2GHz CPU, an AMD Barts Pro Radeon HD 6850 Graphic Processing Unit (GPU) and a 12 GB of random-access memory (RAM). The operating system is Ubuntu Linux 16.04 64-bit version. Built on top of these is a software combination of RStudio and TensorFlow.

The data preprocessing was performed with RStudio, including data segmentation, data transformation, and shallow feature extracting. The details and complete code is also available in R-markdown format [32]. The deep learning model training and testing were conducted with TensorFlow (Version 1.0), and the model was built in Python (Version 2.7) language. TensorFlow is an interface for expressing machine learning algorithms, and an application for executing such algorithms, including training and inference algorithms for deep neural network models. More specifically, the TF.Learn module of TensorFlow was adopted for creating, configuring, training, and evaluating the deep learning model. TF.Learn is a high-level Python module for distributed machine learning inside TensorFlow. It integrates a wide range of state-of-art machine learning algorithms built on top of TensorFlow's low-level APIs for small- to large-scale supervised and unsupervised problems. The details of building deep learning models with TensorFlow are provided online, and some of the trained models are also available [32].

\subsection{Results and Discussion}

There are two evaluation schemes for the activity recognition model, which are a persondependent method and a person-independent, leave-one-out method [17]. For person-dependent evaluation, the data from the same subject are separated to training samples and testing samples. For person-independent evaluation, the data of one or more subjects are excluded from the training process and used for testing. In our study, considering the small number of subjects we have, and in order to compare with a previous study [8], we used the person-dependent method. The classifiers were trained and evaluated for each subject individually. The data of each subject were segmented with a non-overlapping method to avoid over-fitting caused by data duplication in training and testing datasets. Ten percent of the segmented samples were used as testing data, and the remaining samples were used as training data. Sequential selection of samples in time was applied in order to avoid the over-fitting caused by predicting past based on future. All segment lengths were power values of 2 in order to better perform STFT when generating spectrogram images.

These segments were transformed into raw acceleration plots, multichannel plots and spectrogram images, according to the preprocessing methods that were introduced above. For each segment, the 15 shallow features that appear in Table 1 were extracted for each position and axis. Since each segment contains the acceleration data of three axis and seven positions, 315 shallow features were extracted for each segment. The details of data transformation and feature extracting are available [32].

Different deep learning models were built and trained for each combination of the five segmentation options and four data transformation methods. The introduced methods were evaluated for each individual subject. Table 2 presents the aggregated classification results of all 15 subjects, based on different segmentation and transformation combinations. The highest overall accuracy was $97.19 \%$, using the multichannel method based on a segment length of 512 (10.24 s).

Table 2. Overall accuracy (\%) of the four data transformation methods, based on five segmentation options.

\begin{tabular}{ccccc}
\hline Segment Length & Raw Plot & Multichannel & Spectrogram & Spectrogram and Shallow Features \\
\hline 64 & 92.44 & 94.60 & 92.86 & 90.39 \\
128 & 93.05 & 96.14 & 93.37 & 90.42 \\
256 & 93.45 & 96.58 & 93.94 & 92.02 \\
512 & 94.97 & 97.19 & 95.56 & 93.58 \\
1024 & 82.13 & 92.81 & 91.54 & 85.55 \\
\hline
\end{tabular}


The results show that the multichannel method achieved the best performance for all segment lengths. For each of these four transformation methods, the performance improved with the increase of the segment length, from segment length $64(2.56 \mathrm{~s})$ to $512(10.24 \mathrm{~s})$. There is an accuracy decrease from segment length 512 (10.24 s) to 1024 (20.48 s). A possible explanation is the significant drop of training sample numbers. The accuracy of the multichannel method is more stable than other methods, among different segment lengths. This means that the performance variance of the multichannel method is less than that of others, and its classification accuracy is less dependent on segment lengths, which implies that this method is more suitable for short-time HAR tasks. Moreover, the introduction of shallow features did not increase performance as expected. In fact, it slightly decreased performance compared to the spectrogram method. One possible explanation is that the number of shallow features, which was 315, was too many, and they were confused with features extracted by the deep learning models.

With the same data preprocessing method, the classification accuracies of different individuals were different due to the variation of data quality, dataset size, and individual behaviors. Table 3 summarizes the overall classification accuracies of the 15 subjects, based on a segment length of 512 (10.24 s) with the four data preprocessing methods.

Table 3. Variation of overall classification accuracies (\%) of 15 subjects based on a segment length of 512 (10.24 s) with four preprocessing methods.

\begin{tabular}{ccccc}
\hline Subject & Raw Plot & Multichannel & Spectrogram & Spectrogram \& Shallow Features \\
\hline Mean & 95.25 & 97.58 & 95.81 & 93.92 \\
Min. & 92.42 & 93.91 & 91.61 & 88.46 \\
Max. & 97.22 & 99.56 & 98.57 & 97.18 \\
Sd. & 1.72 & 2.11 & 2.35 & 2.74 \\
\hline
\end{tabular}

Leaving out the impact of the segment length, the four models that were based on the segment length of 512 (10.24 s) were compared in detail. Table 4 presents the classification accuracy of each of the eight activities that the four models produced.

Table 4. Performance of each model based on a segment length of 512 (10.24 s). A1: climbing down; A2: climbing up; A3: jumping; A4: lying; A5: running; A6: sitting; A7: standing; and A8: walking.

\begin{tabular}{cccccccccc}
\hline & & A1 & A2 & A3 & A4 & A5 & A6 & A7 & A8 \\
\hline \multirow{3}{*}{ Raw plot } & Precision (\%) & 97.16 & 97.99 & 99.61 & 99.59 & 95.18 & 99.15 & 92.06 & 99.49 \\
& Recall (\%) & 95.41 & 96.89 & 98.78 & 99.18 & 91.40 & 98.53 & 85.24 & 99.08 \\
& Overall Acc. (\%) & & 94.97 & & & $95 \%$ CI: & $(0.9434,0.9556)$ & \\
\hline \multirow{3}{*}{ Multichannel } & Precision (\%) & 97.65 & 97.96 & 99.74 & 99.89 & 96.29 & 99.63 & 96.99 & 99.72 \\
& Recall (\%) & 95.56 & 96.53 & 99.49 & 100.00 & 93.33 & 99.34 & 95.04 & 99.53 \\
& Overall Acc. (\%) & & 97.19 & & & $95 \%$ CI: $(0.9670,0.9763)$ & \\
\hline \multirow{3}{*}{ Spectrogram } & Precision (\%) & 97.65 & 97.23 & 99.92 & 98.60 & 98.84 & 97.47 & 91.18 & 97.76 \\
& Recall (\%) & 95.65 & 96.05 & 100.00 & 97.56 & 98.96 & 96.55 & 82.73 & 96.08 \\
& Overall Acc. (\%) & & 94.56 & & & $95 \%$ CI: $(0.9251,0.9618)$ & \\
\hline \multirow{2}{*}{ Spectrogram \& } & Precision (\%) & 94.92 & 98.25 & 91.51 & 98.60 & 95.92 & 96.60 & 93.39 & 95.38 \\
Shallow features & Recall (\%) & 91.05 & 98.59 & 83.33 & 97.56 & 93.14 & 93.75 & 88.42 & 91.51 \\
& Overall Acc. (\%) & & 93.58 & & & $95 \%$ CI: $(0.9157,0.9512)$ & \\
\hline
\end{tabular}

Regarding to training time, the multichannel method also achieved outstanding performance. As shown in Figure 6, the multichannel model took only 40 min to reach the highest accuracy, whereas the other methods required at least $360 \mathrm{~min}$. This proved that the multichannel method provided the best performance, in this case from both accuracy and training time points of view. 


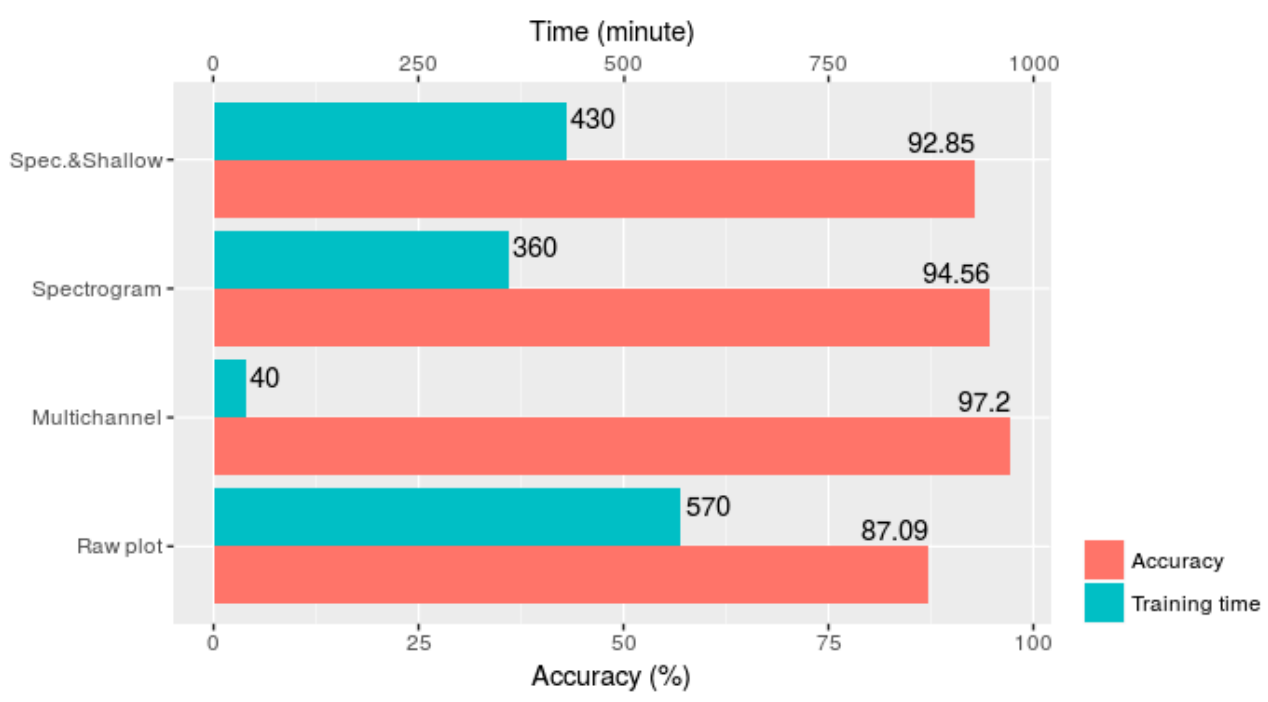

Figure 6. Classification accuracy and training time of the four data transformation methods.

Considering the classification accuracy of each activity, the multichannel method perfectly classified the 68 climbing down (A1) samples, as presented in Table 5. It produced a relatively lower accuracy for running activity (A5), where 5 out of 105 running samples were misclassified as standing activity (A7).

Table 5. Confusion matrix generated by the multichannel model based on a segment length of 512 (A1: climbing down; A2: climbing up; A3: jumping; A4: lying; A5: running; A6: sitting; A7: standing; and A8: walking).

\begin{tabular}{ccccccccc}
\hline \multirow{2}{*}{ Original } & \multicolumn{8}{c}{ Prediction } \\
\cline { 2 - 10 } & A1 & A2 & A3 & A4 & A5 & A6 & A7 & A8 \\
\hline A1 & 68 & 0 & 0 & 0 & 0 & 0 & 0 & 0 \\
A2 & 0 & 78 & 2 & 0 & 1 & 0 & 0 & 1 \\
A3 & 0 & 3 & 22 & 0 & 0 & 0 & 0 & 0 \\
A4 & 0 & 0 & 0 & 81 & 1 & 0 & 0 & 0 \\
A5 & 0 & 6 & 0 & 0 & 98 & 1 & 3 & 0 \\
A6 & 0 & 0 & 0 & 0 & 0 & 92 & 1 & 0 \\
A7 & 0 & 0 & 0 & 1 & 5 & 1 & 86 & 0 \\
A8 & 0 & 1 & 0 & 0 & 0 & 0 & 2 & 100 \\
\hline
\end{tabular}

The classification above is based on the acceleration data that were collected from seven body positions. In real life scenarios, it is difficult to obtain such a complete dataset. Therefore, activity classification using the data from each single position was also undertaken in this study. The combination of segment length 512 (10.24 s) and the multichannel method was used to better compare with the above-mentioned results. Figure 7 shows the overall classification accuracy for the eight activities. The data from the head produced the lowest accuracy $(79.32 \%)$, whereas the data collected from the shin provided the highest accuracy $(90.51 \%)$. This result agrees with practical experience that the movements of the head are more stable than other body positions, whereas the movements of the shin are more closely related to different activities, especially to such dynamic ones such as running, jumping, climbing up, and climbing down. By combining the data from the two positions with the data of highest accuracies, the shin and forearm, an overall accuracy of $93.00 \%$ was achieved. This is close to the result that was obtained based on the data from all of the seven positions, which was $97.20 \%$. 


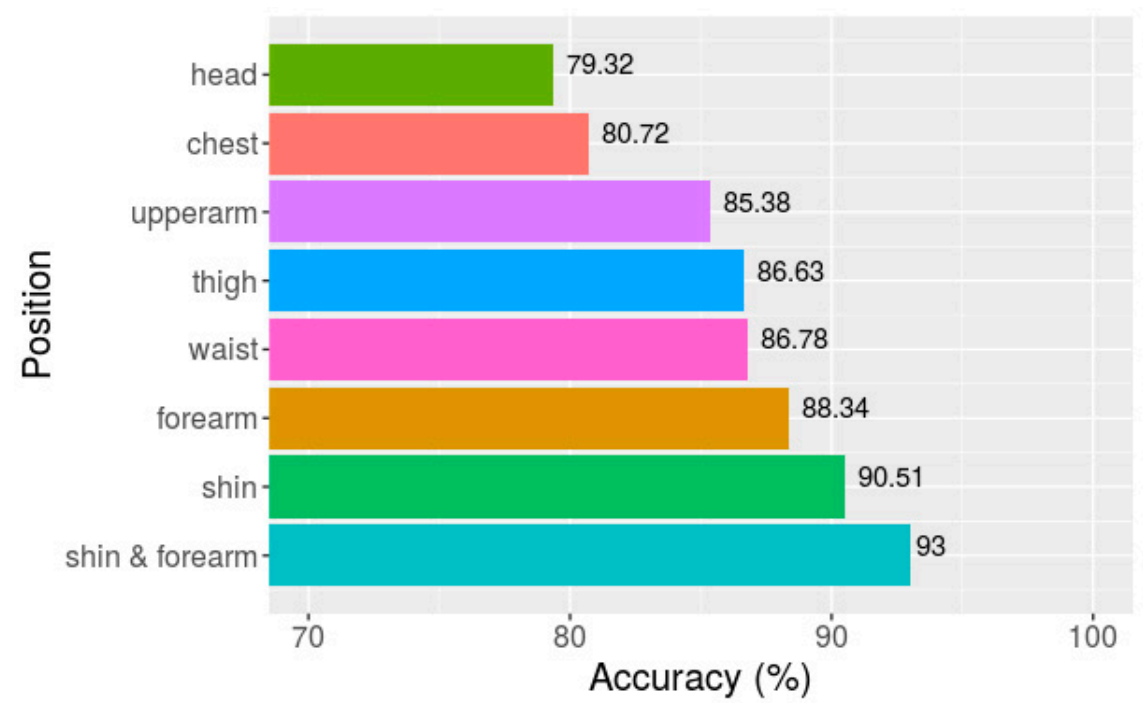

Figure 7. Overall classification accuracies of eight activities based on data from seven single positions and two combined positions.

Compared to other traditional classification techniques, such as ANN, DT, k-NN, NB, SVM, and RF, deep learning methods improved the classification accuracy significantly. Figure 8 shows a comparison of the results achieved by the proposed multichannel deep learning method (marked as DL) based on the segment length of $64(1.28 \mathrm{~s})$ and the results reported in [8], using the same dataset with a similar segment length of one second. It is shown that the deep learning method achieved an overall classification accuracy that was $7.22 \%$ higher than RF.

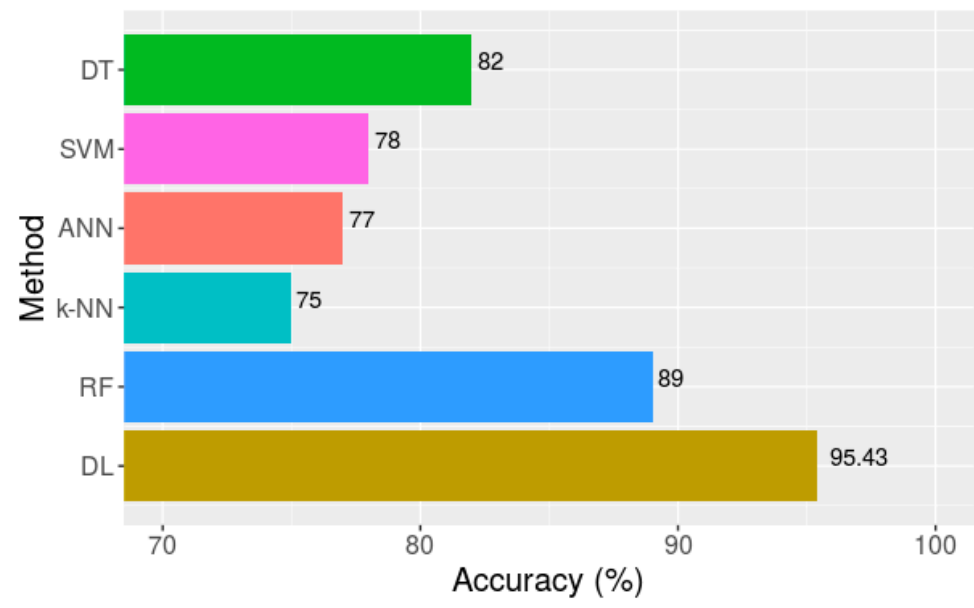

Figure 8. Accuracy of different classification methods [8].

Beside the dataset used above, in order to testify its feasibility, the proposed multichannel data preprocessing method was also applied to another three public HAR datasets, which are WISDM v1.1 (daily activity data collected by a smartphone in a laboratory, with a sampling rate of $20 \mathrm{~Hz}$ ) [33], WISDM v2.0 (daily activity data collected by a smartphone in an uncontrolled environment, with a sampling rate of $20 \mathrm{~Hz}$ ) [34,35], and Skoda (manipulative gestures performed in a car maintenance scenario, with sampling rate of $98 \mathrm{~Hz}$ ) [36]. These datasets were used by Ravì et al. [23], and we used the same segment length as they did, which is a non-overlapping window size of $4 \mathrm{~s}$ (for the Skoda dataset) and $10 \mathrm{~s}$ (for the WISDM v1.1 and WISDM v2.0 datasets). 
The comparison about the per-class precision and recall values obtained by the proposed multichannel transformation method (abbreviated as MCT in the tables) against the results produced by [23] is presented in Table 6 . The result shows that the proposed method outperforms the spectrogram integrated with shallow features method in most activities, except the walking and jogging in the WISDM v1.1 dataset and walking in the WISDM v2.0 dataset. In terms of the multi-sensor Skoda dataset, the proposed method perfectly classified most activities, except the open and close left front door activities. This comparison result reveals that the proposed multichannel method is more suitable for multi-source data, although it can also achieve good results for singular sensor data.

Table 6. Precision (\%) and recall (\%) obtained by the proposed multichannel (MCT) method and existing study [23] in three public datasets.

\begin{tabular}{|c|c|c|c|c|c|c|c|}
\hline \multicolumn{8}{|c|}{ Dataset 1: WISDM v1.1 } \\
\hline & & Walking & Jogging & Sitting & Standing & Upstairs & Downstairs \\
\hline $\begin{array}{l}\text { Ravì et al. } \\
\text { [23] }\end{array}$ & Prec. & 99.37 & 99.64 & 97.85 & 98.15 & 95.52 & 94.44 \\
\hline \multirow{2}{*}{ MCT } & Prec. & 98.34 & 98.11 & 100.00 & 100.00 & 96.14 & 98.44 \\
\hline & Rec. & 97.31 & 97.53 & 100.00 & 100.00 & 93.10 & 97.67 \\
\hline \multicolumn{8}{|c|}{ Dataset 2: WISDM v2.0 } \\
\hline \multirow{2}{*}{$\begin{array}{c}\text { Ravì et al. } \\
\text { [23] }\end{array}$} & Prec. & 98.01 & 88.65 & 87.32 & 85.00 & 82.05 & 97.17 \\
\hline & Rec. & 97.73 & 85.85 & 89.28 & 76.98 & 82.11 & 97.19 \\
\hline \multirow{2}{*}{$\mathrm{MCT}$} & Prec. & 98.76 & 96.85 & 90.25 & 87.03 & 91.02 & 95.85 \\
\hline & Rec. & 97.95 & 94.96 & 82.05 & 75.00 & 85.94 & 94.81 \\
\hline \multicolumn{8}{|c|}{ Dataset 3: Skoda } \\
\hline $\begin{array}{c}\text { Ravì et al. } \\
\text { [23] }\end{array}$ & Rec. & 91.34 & 97.78 & 94.44 & 92.79 & 100.00 & \\
\hline \multirow{3}{*}{ MCT } & Prec. & 100.00 & 99.54 & 100.00 & 100.00 & 80.00 & \\
\hline & Rec. & 100.00 & 100.00 & 100.00 & 100.00 & 60.00 & \\
\hline & & $\begin{array}{l}\text { Close Left Front } \\
\text { Door }\end{array}$ & $\begin{array}{l}\text { Close Both Left } \\
\text { Door }\end{array}$ & $\begin{array}{c}\text { Check Trunk } \\
\text { Gaps }\end{array}$ & $\begin{array}{c}\text { Open and Close } \\
\text { Trunk }\end{array}$ & $\begin{array}{c}\text { Check Steer } \\
\text { Wheel }\end{array}$ & \\
\hline \multirow{2}{*}{$\begin{array}{l}\text { Ravì et al. } \\
\text { [23] }\end{array}$} & Prec. & 88.89 & 92.86 & 98.78 & 100.00 & 93.55 & \\
\hline & Rec. & 80.00 & 94.20 & 97.59 & 98.04 & 100.00 & \\
\hline \multirow{2}{*}{ MCT } & Prec. & 99.18 & 100.00 & 100.00 & 100.00 & 94.44 & \\
\hline & Rec. & 100.00 & 100.00 & 100.00 & 100.00 & 88.89 & \\
\hline
\end{tabular}

\section{Discussions and Conclusions}

In this paper, preprocessing techniques in human activity recognition tasks by deep learning have been considered as a design parameter, and they were shown to be relevant. By comparing different data preprocessing approaches, we came to the following conclusions. Firstly, the length of data segment significantly impacts the final classification accuracy of the deep learning model. The accuracy improves with the increasing of the segment length, and the increasing rate is slower when the segment length is longer. This result agrees with the findings of previous studies that HAR are usually based on data segments of 1 to $10 \mathrm{~s}$. Secondly, four different data transformation methods were compared, and the multichannel method achieved the best performance in both classification accuracy and training time. Unlike the reports of previous studies, we found that the introducing of shallow features did not increase the final accuracy when the experiments were based on a large and multisource dataset. By comparing the classification accuracy based on the data from seven different body positions, it was found that the acceleration data from the shin produced the highest accuracy of $90.51 \%$. A satisfactory accuracy of $93.00 \%$ was achieved by combining the data from the shin and forearm. Moreover, we compared the proposed method against some of other common machine learning methods, based on the same dataset, and it was proven that the deep learning method outperforms others impressively. Finally, we applied the proposed multichannel method to 
three more public datasets, including the car maintenance activity data in a workshop. The results proved that our method can achieve satisfying recognition accuracy. It can help better analyze workers' activities in a factory environment and help integrate people into the cyber-physical structure in an Industry 4.0 context.

Author Contributions: Methodology: X.Z. and J.O.-M.; Formal analysis: X.Z.; Data curation: X.Z. and M.W.; Writing (original draft preparation): X.Z.; Writing (review and editing): J.O.-M.; Supervision: J.O.-M.

Funding: This research received no external funding.

Acknowledgments: The authors thank the support of the China Scholarship Council.

Conflicts of Interest: The authors declare no conflict of interest.

\section{References}

1. Chen, S.; Chen, Y.; Hsu, C. A New approach to integrate internet-of-things and software-as-a-service model for logistic systems: A case study. Sensors 2014, 14, 6144-6164. [CrossRef] [PubMed]

2. Lee, J.; Bagheri, B.; Kao, H. A cyber-physical systems architecture for industry 4.0-based manufacturing systems. Manuf. Lett. 2015, 3, 18-23. [CrossRef]

3. $\mathrm{Xu}, \mathrm{X}$. From cloud computing to cloud manufacturing. Robot. Comput. Integr. Manuf. 2012, 28, 75-86. [CrossRef]

4. Ooi, K.; Lee, V.; Tan, G.W.; Hew, T.; Hew, J. Cloud computing in manufacturing: The next industrial revolution in malaysia? Expert Syst. Appl. 2018, 93, 376-394. [CrossRef]

5. Hao, Y.; Helo, P. The role of wearable devices in meeting the needs of cloud manufacturing: A case study. Robot. Comput. Integr. Manuf. 2017, 45, 168-179. [CrossRef]

6. Putnik, G. Advanced manufacturing systems and enterprises: Cloud and ubiquitous manufacturing and an architecture. J. Appl. Eng. Sci. 2012, 10, 127-134.

7. Gorecky, D.; Schmitt, M.; Loskyll, M.; Zühlke, D. Human-machine-interaction in the industry 4.0 era. In Proceedings of the 12th IEEE International Conference on Industrial Informatics (INDIN), Porto Alegre, Brazil, 27-30 July 2014; pp. 289-294.

8. Sztyler, T.; Stuckenschmidt, H. On-body localization of wearable devices: An investigation of position-aware activity recognition. In Proceedings of the IEEE International Conference on Pervasive Computing and Communications (PerCom), Sydney, Australia, 14-18 March 2016; pp. 1-9.

9. Kwon, Y.; Kang, K.; Bae, C. Unsupervised learning for human activity recognition using smartphone sensors. Expert Syst. Appl. 2014, 41, 6067-6074. [CrossRef]

10. Ward, J.A.; Lukowicz, P.; Troster, G.; Starner, T.E. Activity recognition of assembly tasks using body-worn microphones and accelerometers. IEEE Trans. Pattern Anal. Mach. Intell. 2006, 28, 1553-1567. [CrossRef] [PubMed]

11. Shoaib, M.; Bosch, S.; Incel, O.D.; Scholten, H.; Havinga, P.J. Complex human activity recognition using smartphone and wrist-worn motion sensors. Sensors 2016, 16, 426. [CrossRef] [PubMed]

12. Attal, F.; Mohammed, S.; Dedabrishvili, M.; Chamroukhi, F.; Oukhellou, L.; Amirat, Y. Physical human activity recognition using wearable sensors. Sensors 2015, 15, 31314-31338. [CrossRef] [PubMed]

13. Nakai, D.; Maekawa, T.; Namioka, Y. Towards unsupervised measurement of assembly work cycle time by using wearable sensor. In Proceedings of the IEEE International Conference on Pervasive Computing and Communication Workshops (PerCom Workshops), Sydney, Australia, 14-18 March 2016; pp. 1-4.

14. Koskimaki, H.; Huikari, V.; Siirtola, P.; Laurinen, P.; Roning, J. Activity recognition using a wrist-worn inertial measurement unit: A case study for industrial assembly lines. In Proceedings of the 17th Mediterranean Conference on Control and Automation, Thessaloniki, Greece, 24-26 June 2009; pp. 401-405.

15. Kim, E.; Helal, S.; Cook, D. Human activity recognition and pattern discovery. IEEE Pervasive Comput. 2010, 9, 48-53. [CrossRef] [PubMed]

16. Lee, Y.; Cho, S. Activity recognition with android phone using mixture-of-experts co-trained with labeled and unlabeled data. Neurocomputing 2014, 126, 106-115. [CrossRef]

17. Bulling, A.; Blanke, U.; Schiele, B. A Tutorial on human activity recognition using body-worn inertial sensors. ACM Comput. Surv. 2014, 46, 33. [CrossRef] 
18. Álvarez de la Concepción, M.A.; Soria Morillo, L.M.; Gonzalez-Abril, L.; Ortega Ramírez, J.A. Discrete techniques applied to low-energy mobile human activity recognition. A New approach. Expert Syst. Appl. 2014, 41, 6138-6146. [CrossRef]

19. Clark, C.C.; Barnes, C.M.; Stratton, G.; McNarry, M.A.; Mackintosh, K.A.; Summers, H.D. A review of emerging analytical techniques for objective physical activity measurement in humans. Sports Med. 2016, 47, 439-447. [CrossRef] [PubMed]

20. Hassan, M.M.; Uddin, M.Z.; Mohamed, A.; Almogren, A. A Robust human activity recognition system using smartphone sensors and deep learning. Future Gener. Comput. Syst. 2018, 81, 307-313. [CrossRef]

21. LeCun, Y.; Bengio, Y.; Hinton, G. Deep learning. Nature 2015, 521, 436-444. [CrossRef] [PubMed]

22. Krizhevsky, A.; Sutskever, I.; Hinton, G.E. Imagenet classification with deep convolutional neural networks. In Proceedings of the 25th International Conference on Neural Information Processing Systems, Lake Tahoe, NV, USA, 3-6 December 2012; pp. 1097-1105.

23. Ravì, D.; Wong, C.; Lo, B.; Yang, G. A Deep learning approach to on-node sensor data analytics for mobile or wearable devices. IEEE J. Biomed. Health Inf. 2017, 21, 56-64. [CrossRef] [PubMed]

24. Ronao, C.A.; Cho, S. Human activity recognition with smartphone sensors using deep learning neural networks. Expert Syst. Appl. 2016, 59, 235-244. [CrossRef]

25. Hammerla, N.Y.; Halloran, S.; Ploetz, T. Deep, Convolutional, and Recurrent Models for Human Activity Recognition Using Wearables. 2016. Available online: https://arxiv.org/abs/1604.08880 (accessed on 23 May 2018).

26. Kotsiantis, S.; Kanellopoulos, D.; Pintelas, P. Data preprocessing for supervised leaning. Int. J. Comput. Sci. 2006, 1, 111-117.

27. Zhang, L.; Zhang, L.; Du, B. Deep learning for remote sensing data: A technical tutorial on the state of the art. IEEE Geosci. Remote Sens. Mag. 2016, 4, 22-40. [CrossRef]

28. Lara, O.D.; Labrador, M.A. A Survey on human activity recognition using wearable sensors. IEEE Commun. Surv. Tutor. 2013, 15, 1192-1209. [CrossRef]

29. Preece, S.J.; Goulermas, J.Y.; Kenney, L.P.; Howard, D. A comparison of feature extraction methods for the classification of dynamic activities from accelerometer data. IEEE Trans. Biomed. Eng. 2009, 56, 871-879. [CrossRef] [PubMed]

30. Sejdić, E.; Djurović, I.; Jiang, J. Time-frequency feature representation using energy concentration: An overview of recent advances. Digit. Signal Process. 2009, 19, 153-183. [CrossRef]

31. LeCun, Y.; Bengio, Y. Convolutional networks for images, speech, and time series. In The Handbook of Brain Theory and Neural Networks; MIT Press: Cambridge, MA, USA, 1998; pp. 255-258.

32. Zheng, X.; Ordieres, J. Step-by-Step Introduction to Acceleration Data Classification Using Deep Learning Methods. 2017. Available online: https://www.researchgate.net/publication/317180890 (accessed on 23 May 2018).

33. Kwapisz, J.R.; Weiss, G.M.; Moore, S.A. Activity recognition using cell phone accelerometers. ACM SigKDD Explor. Newslett. 2011, 12, 74-82. [CrossRef]

34. Lockhart, J.W.; Weiss, G.M.; Xue, J.C.; Gallagher, S.T.; Grosner, A.B.; Pulickal, T.T. Design considerations for the WISDM smart phone-based sensor mining architecture. In Proceedings of the Fifth International Workshop on Knowledge Discovery from Sensor Data, San Diego, CA, USA, 21-24 August 2011; pp. 25-33.

35. Weiss, G.M.; Lockhart, J.W. The Impact of Personalization on Smartphone-Based Activity Recognition; AAAI Technical Report WS-12-05; Fordham University: New York, NY, USA, 2012; pp. 98-104.

36. Zappi, P.; Lombriser, C.; Stiefmeier, T.; Farella, E.; Roggen, D.; Benini, L.; Troster, G. Activity recognition from on-body sensors: Accuracy-power trade-off by dynamic sensor selection. Lect. Notes Comput. Sci. 2008, 4913, 17-33.

(C) 2018 by the authors. Licensee MDPI, Basel, Switzerland. This article is an open access article distributed under the terms and conditions of the Creative Commons Attribution (CC BY) license (http://creativecommons.org/licenses/by/4.0/). 


\subsection{Publication 4}

- Title: Activity-aware essential tremor evaluation using deep learning method based on acceleration data

- Authors: Xiaochen Zheng, Alba Vieira, Sergio Labrador Marcos, Yolanda Aladro, Joaquín Ordieres-Meré

- Publication date: 2018/8/8

- Journal: Parkinsonism E related disorders

Impact Factor: $4.721(2017) / 4.855$ (5 year)

Quartile: Q1

ISSN: $1353-8020$

Publisher: ELSEVIER SCI LTD, OXFORD OX5 1GB, OXON, ENGLAND

- DOI: https://doi.org/10.1016/j.parkreldis.2018.08.001

- Copyright statement: This article is published in Open Access journals. According to the publisher's policy, "All articles published gold open access will be immediately and permanently free for everyone to read and download." 4

\footnotetext{
${ }^{4}$ https://www.elsevier.com/journals/parkinsonism-and-related-disorders/1353-8020/open-access-options
} 


\title{
Activity-aware essential tremor evaluation using deep learning method based on acceleration data
}

\author{
Xiaochen Zheng ${ }^{\mathrm{a}}$, Alba Vieira ${ }^{\mathrm{b}}$, Sergio Labrador Marcos ${ }^{\mathrm{b}}$, Yolanda Aladro ${ }^{\mathrm{b}}$, \\ Joaquín Ordieres-Merée,* \\ a Department of Industrial Engineering, Universidad Politécnica de Madrid, Madrid, Spain \\ ${ }^{\mathrm{b}}$ Neurology Service, Hospital Universitario de Getafe, Getafe, Madrid, Spain
}

\section{A R T I C L E I N F O}

\section{Keywords:}

Essential tremor

Human activity recognition

Deep learning

Convolutional neural network

Blockchain

IoTA

\begin{abstract}
A B S T R A C T
Background: Essential tremor (ET), one of the most common neurological disorders is typically evaluated with validated rating scales which only provide a subjective assessment during a clinical visit, underestimating the fluctuations tremor during different daily activities. Motion sensors have shown favorable performances in both quantifying tremor and voluntary human activity recognition (HAR).

Objective: To create an automated system of a reference scale using motion sensors supported by deep learning algorithms to accurately rate ET severity during voluntary activities, and to propose an IOTA based blockchain application to share anonymously tremor data.

Method: A smartwatch-based tremor monitoring system was used to collect motion data from 20 subjects while they were doing standard tasks. Two neurologists rated ET by Fahn-Tolosa Marin Tremor Rating Scale (FTMTRS). Supported by deep learning techniques, activity classification models (ACMs) and tremor evaluation models (TEMs) were created and algorithms were implemented, to distinguish voluntary human activities and evaluate tremor severity respectively.

Result: A practical application example showed that the proposed ACMs can classify six typical activities with high accuracy $(89.73 \%-98.84 \%)$ and the results produced by the TEMs are significantly correlated with the FTMTRS ratings of two neurologists $\left(r_{1}=0.92, \mathrm{p}_{1}=0.008 ; \mathrm{r}_{2}=0.93, \mathrm{p}_{2}=0.007\right)$.

Conclusion: This study demonstrated that motion sensor data, supported by deep learning algorithms, can be used to classify human activities and evaluate essential tremor severity during different activities.
\end{abstract}

\section{Introduction}

Essential tremor (ET) is one of the most common neurological disorders affecting $4.6-6.3 \%$ of adult above the age of 60-65 [1]. It mainly affects the upper limbs with the consequent interference in basic activities of daily living [2]. Tremor analysis during daily living is crucial in order to better understand the patient status and to evaluate treatment effects [3]. In clinical practice, ET is usually assessed by several tremor validated rating scales [4-6]. Although, these rating scales have demonstrated clinical utility, they require the presence of a clinician for scoring. Their results are subject to clinical judgment and show significant intra and inter explorer variability and cannot reflect tremor fluctuation patterns throughout the day during different daily activities [3,7].

ET shows a frequency between 4 and $12 \mathrm{~Hz}$, which is higher than the frequency of normal voluntary human movements $[8,9]$. The feasibility of detecting and evaluating tremor using motion sensor data has been proved $[3,10,11]$. The development of wearable technology provides a convenient method to collect motion data. The study presented in Ref. [12] has verified the practicability of using a smartwatch to analyze and diagnose tremor and the study of [13] demonstrated the feasibility of continuous monitoring of ET for long period during daily activities.

Most ET patients show both kinetic and postural tremor [1] and the severity of tremor varies when patients conduct different activities $[3,14,15]$. The tremor evaluation methods based on motion sensors presented in most of previous studies, like [12,13], only focused on the tremor itself without considering the background activities. The evaluation results produced by those methods cannot reflect the tremor fluctuation among different daily activities. Because of such variability and the scarcity in available datasets, the research in this field remains limited.

\footnotetext{
* Corresponding author.

E-mail address: j.ordieres@upm.es (J. Ordieres-Meré).
} 
Deep learning is a paradigm of machine learning that enables computational models to learn representations of data with multiple levels of abstraction [16]. Many studies have proved that the use of deep learning can improve performance in a range of applications [16-18]. As a powerful feature extraction mechanism, deep learning has also been applied to human activity recognition (HAR) and achieved satisfying performance [19,20].

In addition, the blockchain technology brings the capability to keep public records of data, including the encrypted id of the patient to preserve anonymity, enabling to build up a system helping the research.

The goal of this study is to demonstrate the feasibility of using the acceleration data supported by deep learning algorithms, not only to recognize typical human daily activities, but also to accurately rate tremor severity during each specific activity in an automatic way. In addition, it aims to propose a blockchain like solution based on IoTA (Internet of Things Application), capable of sharing anonymous patient related tremor data helping to consolidate the research. Blockchain is a continuously growing list of records, called blocks, which are linked and secured using cryptography. Each block contains a cryptographic hash of the previous block, a timestamp, and transaction data. The utility of blockchain in our context is to provide trust and common understanding of data without dependences form a central authority or specific hardware. IoTA allows to implement cost free transactions with easier trust schema.

\section{Material and methods}

This study is approved by the ethics committee of the Getafe University Hospital (Madrid, Spain) and is conducted in accordance with the Declaration of Helsinki of the World Medical Association. All participant patients have signed an informed consent.

\subsection{Subject}

Twenty patients with essential tremor, 11 males and 9 females, mean age $63.0 \pm 13.5$ years (range 38-81) and tremor evolution time $10.35 \pm 7.09$ years, were include. All showed tremor in both upper limbs with different tremor leves, 4 of them associated cephalic tremor. The $75 \%$ of the patients had a family history of ET. None showed tremor of rest. Finally, in 3 a single-photon emission computed tomography using I-ioflupane was performed to exclude other types of tremor due to some atypical clinical characteristics. Eleven were under tremor therapy, two with primidone, six with propranolol, one both drugs, and two with clonazepam. Medication for ET was maintained during the experiment.

In order to compare the data five healthy subjects, mean age years $36.4 \pm 10.3$ (range 28-54), 3 males and 2 females, were included.

\subsection{Procedures of experiment}

The standardized experiment was designed on the basis of the FahnTolosa Marin Tremor Rating Scale (FTMTRS) tasks that evaluate upper limbs tremor severity. The repetition numbers of the tasks were mainly based on the duration time of each task and each repetition. The target was to keep the overall duration of each task more or less the same while considering the body conditions of the patients to avoid muscle fatigue. Under the supervision of at least two neurologists, and wearing the watch firmly on the more affected wrist, patients perform the standardized tasks guided by a video. ${ }^{1}$ The tasks include:

A1) Keep both arms horizontally extended for $20 \mathrm{~s}$ and then relaxed on the lap for $10 \mathrm{~s}$. This task is repeated three times.

A2) Repeat the finger-nose test (touching the nose with the index

\footnotetext{
${ }^{1}$ Available online: https://www.researchgate.net/publication/325923937.
}

finger with the arm raised to $\left.90^{\circ}\right) 10$ times, and then keep the hands relaxed on the lap for $10 \mathrm{~s}$. This task is repeated twice.

A3) Write "THIS IS A SAMPLE OF MY WRITING", and then keep both hands relaxed on the lap for $10 \mathrm{~s}$. This task is repeated three times.

A4) Draw a large and a small spirals, and three lines sequentially with a 10-s rest between every two drawings. This task is repeated twice.

A5) Pour a full glass of water into another empty cup and then pour back for three times, and then rest for $10 \mathrm{~s}$. This task is repeated three times.

A6) Simulate the action of drinking a full glass of water five times and then resting for $10 \mathrm{~s}$. This task is repeated twice.

More details are available in the instructional video ${ }^{1}$.

The experiments were videotaped for subsequent analysis and tremor scoring by two neurologists in a blind way. Each item was rated on a scale from 0 to 4 (no tremor to severe).

\subsection{Hardware and software}

A portable human movement monitoring system has been previously developed [21]. This three-layer system is composed of a smartwatch, which contains a tri-axis accelerometer and Bluetooth 4.0, for recording the user's arm movement data; an Android smartphone for receiving data from smartwatch and uploading them to a remote server; and a cross-platform document-oriented NoSQL MongoDB database on remote server for data storage and analysis. The information collected using this system includes three-axis arm movement acceleration values. The feasibility of analyzing tremor using data collected from a smartwatch has been validated in a previous study [22].

All movement data were recorded with frequency of $25 \mathrm{~Hz}$ and uploaded to the remote server in real time. Fig. 1 shows an example of the acceleration data over time during the experiment of an ET patient. Since rest tremor is not habitual of ET [3] and was not found in our subjects, it was excluded and treated as noise. As shown in Fig. 1, we define three components of the raw acceleration data. The six standard activities (A1 - A6) are individually considered as pure actions. There are gaps between those activities, which are defined as the remaining part of the signal between two pure activities, including the resting periods and other noises during the experiments. Transit means the transition between a pure activity and a rest section.

The target of this study is to identify the pure activities from the raw signal by activity classification models (ACMs) and classify them into different tremor levels with tremor evaluation models (TEMs). Fig. 2 demonstrates the proposed framework for activity classification and tremor evaluation.

All data preprocessing and model training in this study were implemented with a computer equipped with a 4-core Intel Core i5-4460 $3.2 \mathrm{GHz}$ CPU and a $12 \mathrm{~GB}$ of RAM memory. The operating system is Ubuntu Linux 16.04 64-bit version.

Data preprocessing is performed with RStudio. The deep learning model training and testing are conducted with TensorFlow (Version 1.0) and the model was built in Python (Version 2.7) language. TensorFlow (TF) is an interface for expressing machine learning algorithms, and an application for executing such algorithms, including training and inference algorithms for deep neural network models [23]. More specifically, the TF.Learn module of TensorFlow was adopted for creating, configuring, training and evaluating the deep learning model. TF.Learn is a high-level Python module for distributed machine learning inside TensorFlow. It integrates a wide range of state-of-art machine learning algorithms built on top of TensorFlow's low level APIs for small to large-scale supervised and unsupervised problems [24]. The complete code of data preprocessing with RStudio and building deep learning models with TensorFlow are provided in Appendix 1 of the online version. 
$X$. Zheng et al.

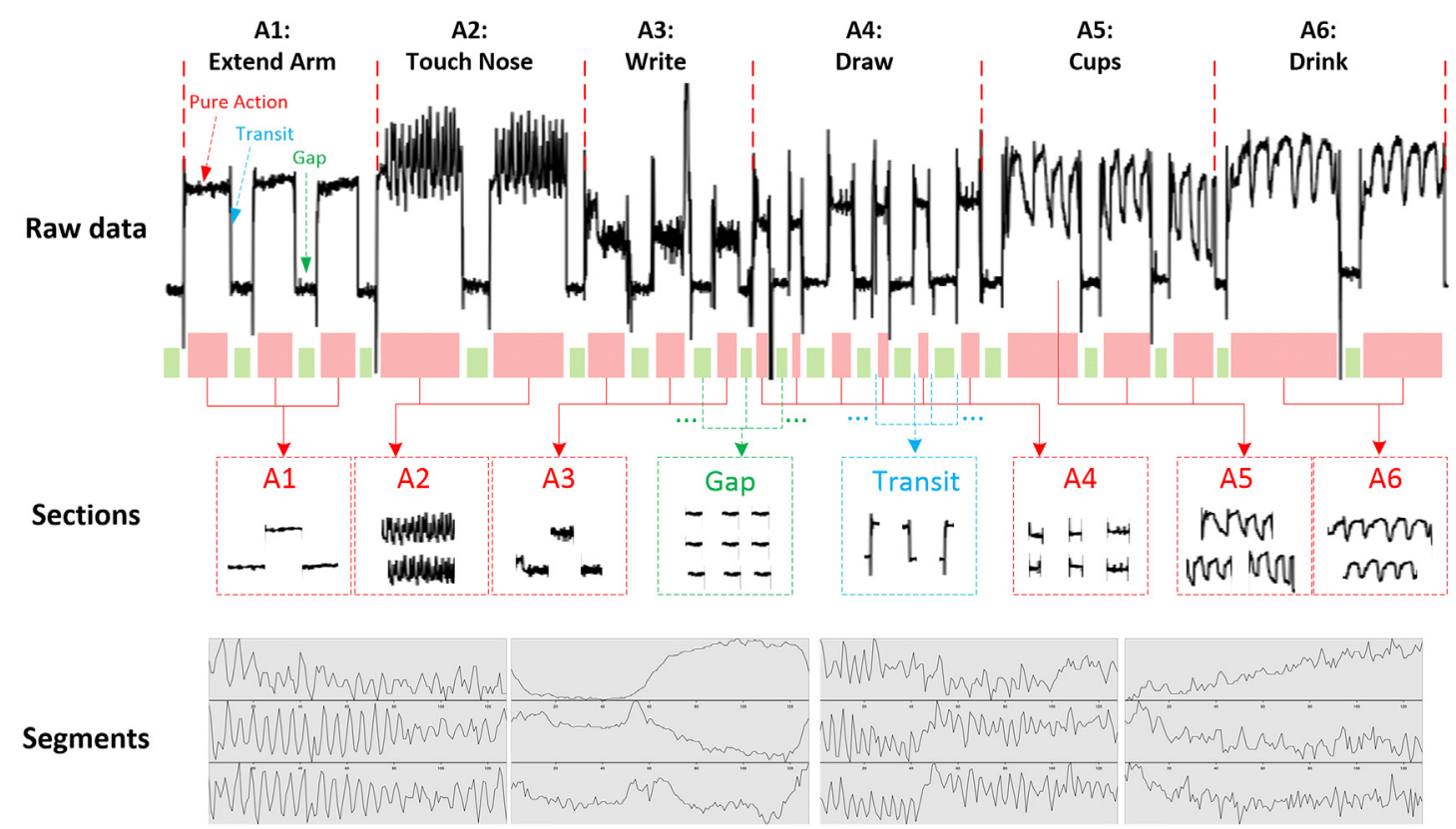

Fig. 1. Example of raw acceleration data and the segmentation process.

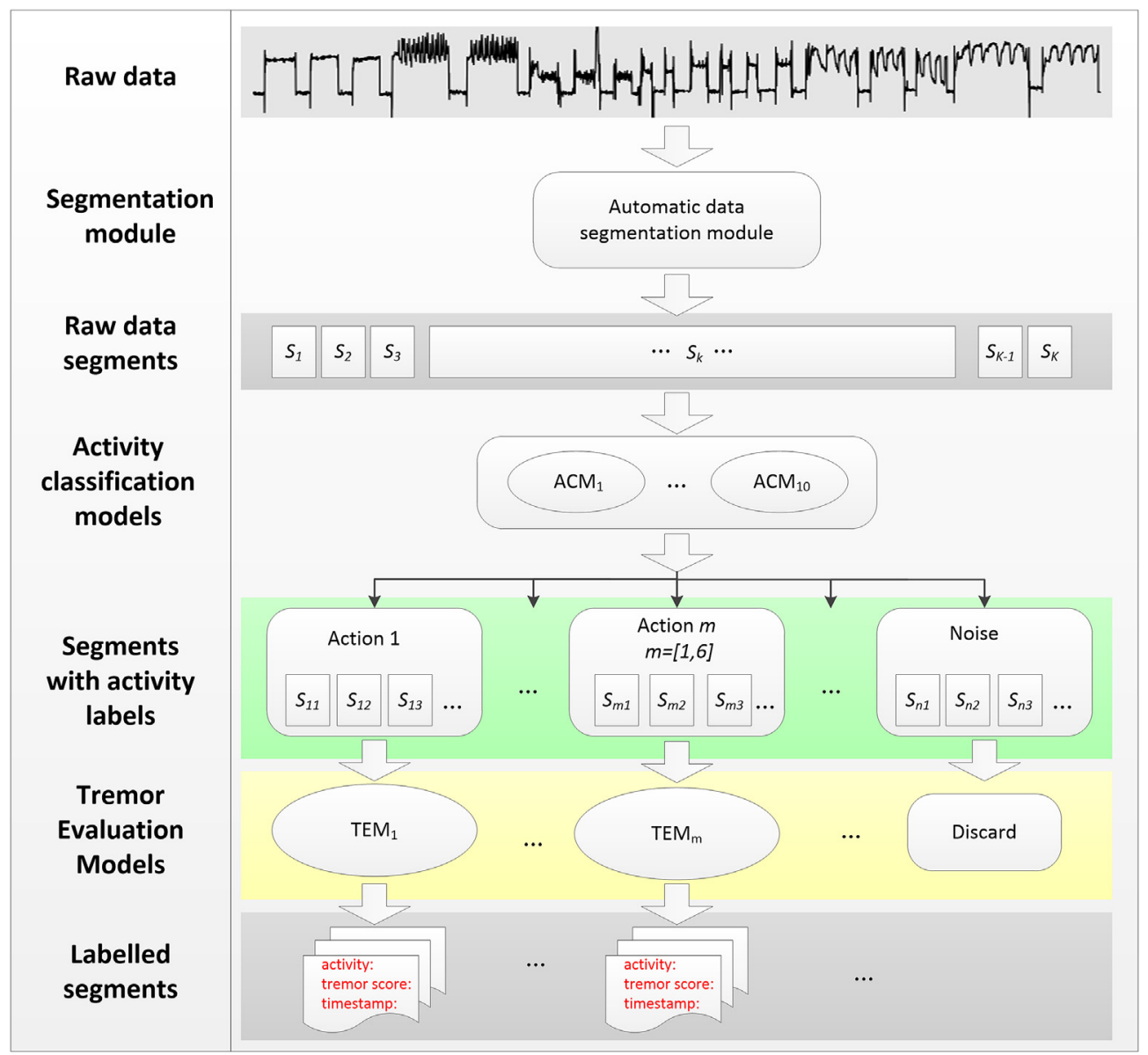

Fig. 2. The proposed framework for activity classification and tremor evaluation.

The IoTA system is an open source permission less public distributed ledger that utilizes a novel invention, called a "Tangle", at its core $[25,26]$. The Tangle is a new data structure based on a Directed Acyclic Graph (DAG). Each participant in the network that wants to make a transaction has to actively participate in the consensus of the network by approving the two past transactions. This attestation ensures that the whole network achieves consensus on the current state of approved transactions, and it enables a variety of significant features like.

- Scalability: IOTA can achieve high transaction throughput thanks to 
parallelized validation of transactions with no limit as to the number of transactions that can be confirmed in a certain interval

- No Transaction Fees: IOTA has no transaction fees.

- Decentralization: IOTA has no miners. Every participant in the network that is making a transaction, actively participates in the consensus. As such, IOTA is more decentralized than any Blockchain.

- Quantum-immunity: IOTA utilized a newly designed trinary hash function called Curl, which is quantum immune (Winternitz signatures)

We have used one light IoTA node from the public repository (https://iotanode.host/), and segments and labels have been encapsulated in transactions. Then, they have been submitted by the software module to the IoTA tangle in an automatic way.

\subsection{Data preprocessing}

Data preprocessing includes data labeling and data segmentation. The raw data collected during the experiments are without labels. In order to use them to train the classification models, these raw data need to be properly segmented and labeled with the following steps.

First, the raw data of each subject were roughly partitioned into 8 groups, including 6 pure activity groups, a transit group and a gap group as shown in Fig. 1. Each group contains several data sections (different from the data segments below) with their own labels. For example, in Figs. 1 and 72 data sections can be obtained, including 19 pure activity sections, 19 gaps and 38 transit sections.

Then, all of these data sections (except the transit sections) are equally segmented into small segments using the sliding window method, which is also known as overlapping window method. This method is used because it is able to handle transitions more accurately and produce more training samples [27]. Exceptionally, the transit sections are segmented with the same window length manually, because their lengths are too short to apply the sliding window method.

The segmentation method is presented below:

Suppose a data section $A$.

$\mathrm{A}=\left\{v_{1}, v_{2}, \ldots, v_{n}\right\}$, where $n$ is the length of the section and $v_{i}$ represents the $i_{t h}$ data record in the signal and $v_{i}=\left\{x_{i}, y_{i}, z_{i}\right\}$, where $x_{i}, y_{i}, z_{i}$ represents the acceleration values on the three directions of the movement.

$A$ is segmented by a sliding window with length $W$, overlapping length $R$.

Then the $k^{\text {th }}$ segment can be represented by:

$S_{k}=\left[v_{(R *(k-1)+1)}, v_{(R *(k-1)+1+W)}\right]$, where $S_{k}$ represents the segment data array and $k=\left[1, \operatorname{round}\left(\frac{n-W}{R}\right)\right]$.

After the segmentation, the raw data will be separated into small segments and every segment contains the tri-axis acceleration data, an activity label and a tremor score label. Some examples of these segments corresponding to different activities are shown on the bottom of Fig. 1.

\subsection{Model building}

As shown in Fig. 2, two types of models, ACMs and TEMs, are built and trained. They are the key modules of the proposed system. In this study, the deep Convolutional Neural Network (CNN) architecture $[16,28]$ is used to build both ACMs and TEMs. CNN is one of the most popular deep learning approaches, which utilize layers with convolving filters that are applied to local features. The capacity of CNNs can be controlled by varying their depth and breadth, and they also make strong and mostly correct assumptions about the nature of the data samples. Compared to standard feed-forward neural networks with similarly-sized layers, CNNs have much fewer connections and parameters and so they are easier to train, while their theoretically-best performance is likely to be only slightly worse [18]. CNN has been wildly applied and has produced satisfying results in a number of studies [29-31. The general structure of ACM and TEM in our study is similar but the inputs and outputs are different.

For ACMs, the inputs are all the samples obtained during data preprocessing, including pure actions, gaps and transit segments and correspondingly, the outputs of ACMs are the 8 activity classes. As for the TEMs, only the samples obtained from pure action data are used as inputs while the gaps and transit segments are excluded. The outputs of TEMs are the tremor severity scores. Evaluation models for each pure action are built and trained separately.

\subsection{System application}

In practical application, the raw data of a new patient will be sequentially segmented with the same length as during training process, but without overlapping. Segmented samples will then pass through the prepared ACMs and be classified into 8 classes. Afterwards, samples which are classified as pure activities will pass through their corresponding TEMs and be classified into different tremor levels. In the end, samples classification results which belong to the same pure activity will be summarized to reflect tremor severity during corresponding activity.

\section{Results}

\subsection{Data segmentation}

The data of 5 patients were selected for validation during the application step and the rest were used to train classification models. During data segmentation process, several segment lengths were used, including $2.56 \mathrm{~s}$ (64 data samples), $5.12 \mathrm{~s}$ (128 data samples), $7.68 \mathrm{~s}$ (192 data samples) and $10.24 \mathrm{~s}$ (256 data samples). Theoretically, the classification accuracy will be higher with longer segments. However, in some activities in our experiment, the duration is very short, for example, the spirals drawing (less than $10 \mathrm{~s}$ ). In this case, long segment may cover more than one activities and resulting in misclassification. Therefore, the segment length $5.12 \mathrm{~s}$ (128 data samples) was adopted for further model training and applying.

\subsection{Activity classification result}

The 10-fold cross-validation method was used to train both the ACMs and TEMs. All segmented data samples were separated into 10 parts randomly and equally. During each training round, one of these 10 parts was selected as validation data and the rest as training data and then started a new round until all parts have been used once as testing data.

For the training of ACMs, 6640 samples were produced with the sliding window method during the segmentation process. The segment length was $5.12 \mathrm{~s}$ (128 data samples) and the overlapping length was $1.28 \mathrm{~s}$ (32 data samples). For the training of TEMs, 5630 samples were generated based on the pure activity data using the same segment and overlapping lengths as ACMs. More specifically, the sample numbers for the 6 activities (A1 - A6) were: 920, 840, 990, 580, 1300 and 1000 respectively. The maximum, minimum and average accuracies obtained by the 10-fold ACMs and TEMs are presented in Table 1 .

As shown in Table 1, the proposed ACMs could recognize most of the predefined activities with high accuracy (mean $0.95 \pm 0.03 \mathrm{SD}$ ). The proposed TEMs also produced high accuracy for tremor severity evaluation (mean $0.90 \pm 0.06 \mathrm{SD}$ ).

In order to compare performance of tremor classification between the proposed activity-aware models and the traditional non-activityaware based models, the samples of all 6 activities were mixed up and used to train tremor classification models without considering activity differences. As shown in Table 1, tremor classification accuracy based 
Table 1

Classification accuracies obtained by ACMs and TEMs during the training process (A1: extend arm; A2: touch nose; A3: write sentences; A4: draw spirals; A5: pour water; A6: simulate drinking).

\begin{tabular}{|c|c|c|c|c|c|c|c|c|}
\hline & & A1 & $\mathrm{A} 2$ & A3 & A4 & A5 & A6 & Mixed \\
\hline \multirow[t]{3}{*}{ ACM } & Max & 0.9819 & 1.00 & 0.9956 & 0.9597 & 0.9282 & 0.9772 & - \\
\hline & Min & 0.9191 & 0.9725 & 0.9703 & 0.8170 & 0.8137 & 0.9288 & - \\
\hline & Average & 0.9632 & 0.9884 & 0.9811 & 0.9301 & 0.8973 & 0.9510 & - \\
\hline \multirow[t]{3}{*}{ TEM } & Max & 0.9674 & 0.9643 & 0.9900 & 0.8966 & 0.8855 & 0.9806 & 0.8952 \\
\hline & Min & 0.8913 & 0.8929 & 0.9091 & 0.7719 & 0.5846 & 0.9029 & 0.7890 \\
\hline & Average & 0.9315 & 0.9440 & 0.9404 & 0.8564 & 0.8030 & 0.9517 & 0.8544 \\
\hline
\end{tabular}

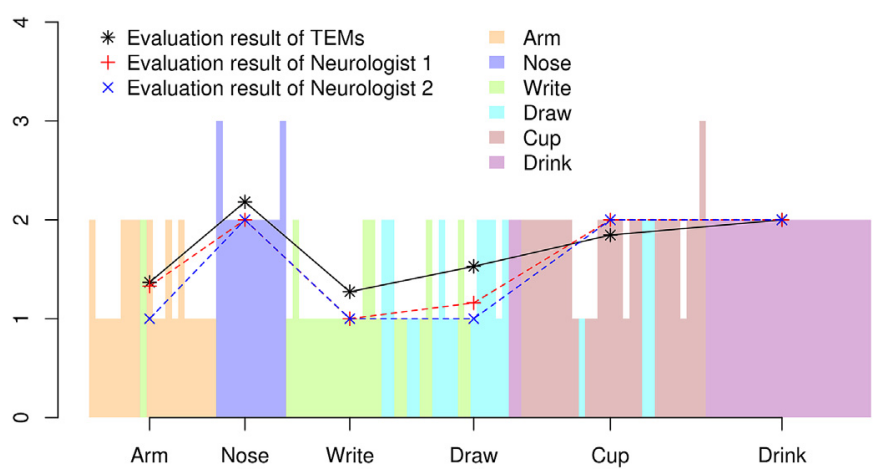

Fig. 3. Tremor evaluation results of one patient obtained by TEMs and two neurologists (the vertical axis represents tremor grades and the horizontal axis represents the action segments.).

on every single activity except A5 (Pour water, above 0.86 ) is higher than general accuracy when mixed up all activities (0.85).

\subsection{Application result}

Data from the same 5 patients were excluded during the model training process and used to verify the performance of the trained models in the practical application. These data were segmented into segments with the same length as in the model training process, $5.12 \mathrm{~s}$ (128 data samples). The $10 \mathrm{ACMs}$ trained before were applied to these segments and classified them into 8 classes. The activity classification results during practical application were summarized on the basis of the outputs of the 10 ACMs. When there are different classification results for the same segment, the mode of the 10 results were adopted.

Based on the activity classification results, the segments which were classified as the 6 pure activities were used as input of the TEMs. These segments were classified into different tremor levels. For each segment, when classification results of the 10 TEMs were different, the mode statistic of the 10 results was adopted. Classification results of the segments which belong to the same data section (a continuous activity) were taken average to represent the score of this activity. Fig. 3 shows evaluation results of the six activities from one patient produced by TEMs and by two neurologists. The classified segments were plotted according to their original sequence in time domain. The activity classification results, which are produced by ACMs, are represented by six different colors and the tremor evaluation results, which are produced by TEMs, are represented by the height of the bars. The results show that the ACMs correctly recognized most of the activities, except that some "draw" segments were misclassified as "write". Significant correlations $\left(r_{1}=0.92, p_{1}=0.008 ; r_{2}=0.93, p_{2}=0.007\right)$ were obtained between evaluation results produced by the TEMs and two neurologist's FTMTRS scores. Since each activity was repeated several times, the average FTMTRS scores of all repeats were adopted to compare with the TEMs results. The tremor level of different repeats for the same activity may differ according to the observation during the experiments and this is also verified by the neurologists' evaluations.
For example, the tremor level during the second repeat of the "arm" activity was higher than the first and third repeats. Correspondingly, the evaluation scores of these three repeats are 1, 2 and 1 respectively.

As a matter of convenience, one specific IoTA address to send all the transactions has been defined:'GEKMPUCSIRYYYNKLMFQDMJIIFAEJFHRUNWSATCMMLDNUBIIROAVPZVDSVOQBKCNVIOJKCRAPHOYOR9YSZ'. Therefore, blocks can be retrieved from different locations and be decrypted to recover the underlying json data structure.

\section{Discussion}

This study proposed an accurate evaluation system of activity-aware ET. Compared with previous studies, we obtain a deeper level of tremor evaluation. The proposed system not only focuses on tremor itself, but also takes into account the background activities when tremor occurred. The deep learning techniques are used twice in the system to classify human activities and evaluate tremor. The proposed ACMs could recognize predefined human activities with high accuracy. The activity-aware tremor evaluation results show strong correlation with the ratings of the neurologists. This correlation is stronger than that obtained in previous studies [3,4,13], which didn't consider the impact of background activities. It means that the introducing of activity classification can increase the accuracy of tremor severity evaluation against the traditional non-activity-aware models.

The output of the proposed system is a sequence of blocks of information, where each block contains the timestamp, gender, age, a numeric identification (ID) making it possible to track the record in reference to the hospital clinic identification, as well as the action and its score according to FTMTRS. In order to foster the research about ET, this paper proposes to share the produced data blocks in a safety way, preserving anonymity. It has been decided to use blockchain like IOTA distributed ledger to share them. Therefore, a light server has been installed through Docker technology, and blocks where encrypted and encapsulated using the benefits of Wallet and cryptocurrency. The central idea is to 'send' transactions where the value is 0 , the tag refers to the ET topic and the hierarchical geography references.

The implemented IoTA solution gets benefit from the public tag that can be additionally used to attach identification for the Hospital and Country codes. In this way, researchers can share and access to wider ET related information, helping to understand individual evolution in both, short and long terms. Indeed, the direct and non-restricted access to such information can be seen as a driver for getting high consensus level as training dataset will be larger. Because of the proposed models are based on collected data, classifiers will improve their accuracy based on the increase of samples.

\section{Appendix A. Supplementary data}

Supplementary data related to this article can be found at https:// doi.org/10.1016/j.parkreldis.2018.08.001.

\section{References}

[1] E.D. Louis, J.J. Ferreira, How common is the most common adult movement disorder? Update on the worldwide prevalence of essential tremor, Mov. Disord. 25 (2010) 534-541.

[2] W.J. Elias, B.B. Shah, Tremor. JAMA 311 (2014) 948-954.

[3] D.A. Heldman, J. Jankovic, D.E. Vaillancourt, J. Prodoehl, R.J. Elble, J.P. Giuffrida, Essential tremor quantification during activities of daily living, Park. Relat. Disord. 17 (2011) 537-542.

[4] G. Mostile, J.P. Giuffrida, O.R. Adam, A. Davidson, J. Jankovic, Correlation between Kinesia system assessments and clinical tremor scores in patients with essential tremor, Mov. Disord. 25 (2010) 1938-1943.

[5] S. Fahn, E. Tolosa, C. Marin, Clinical Rating Scale for Tremor. Parkinson's Disease and Movement Disorders vol. 2, (1993), pp. 271-280.

[6] F. Hopfner, D. Haubenberger, W.R. Galpern, K. Gwinn, A. Van't Veer, S. White, et al., Knowledge gaps and research recommendations for essential tremor, Park. Relat. Disord. 33 (2016) 27-35.

[7] G. Rigas, A.T. Tzallas, M.G. Tsipouras, P. Bougia, E.E. Tripoliti, D. Baga, et al., 
Assessment of tremor activity in the Parkinson's disease using a set of wearable sensors. Information Technology in Biomedicine, IEEE Transactions on 16 (2012) $478-487$.

[8] R.J. Elble, Essential tremor frequency decreases with time, Neurology 55 (2000) $1547-1551$

[9] B. Thanvi, N. Lo, T. Robinson, Essential tremor-the most common movement disorder in older people, Age Ageing 35 (2006) 344-349.

[10] N. Mamorita, T. Iizuka, A. Takeuchi, M. Shirataka, N. Ikeda, Development of a system for measurement and analysis of tremor using a three-axis accelerometer, Method Inf. Med. 48 (2009) 589-594.

[11] R.A. Joundi, J. Brittain, N. Jenkinson, A.L. Green, T. Aziz, Rapid tremor frequency assessment with the iPhone accelerometer, Park. Relat. Disord. 17 (2011) 288-290.

[12] D.J. Wile, R. Ranawaya, Z.H. Kiss, Smart watch accelerometry for analysis and diagnosis of tremor, J. Neurosci. Meth. 230 (2014) 1-4.

[13] X. Zheng, A.V. Campos, J. Ordieres-Meré, J. Balseiro, S.L. Marcos, Y. Aladro, Continuous monitoring of essential tremor using a portable system based on smartwatch, Front. Neurol. 8 (2017).

[14] C. Pulliam, S. Eichenseer, C. Goetz, O. Waln, C. Hunter, J. Jankovic, et al., Continuous in-home monitoring of essential tremor, Park. Relat. Disord. 20 (2014) $37-40$.

[15] N. Schuhmayer, C. Weber, M. Kieler, B. Voller, W. Pirker, E. Auff, et al., Taskdependent variability of essential tremor, Park. Relat. Disord. 41 (2017) 79-85.

[16] Y. LeCun, Y. Bengio, G. Hinton, Deep learning, Nature 521 (2015) 436-444.

[17] M. Zeng, L.T. Nguyen, B. Yu, O.J. Mengshoel, J. Zhu, P. Wu, et al., Convolutional Neural Networks for Human Activity Recognition Using mobile Sensors, (2014), pp. 197-205.

[18] A. Krizhevsky, I. Sutskever, G.E. Hinton, Imagenet Classification with Deep Convolutional Neural Networks, (2012), pp. 1097-1105.

[19] D. Ravì, C. Wong, B. Lo, G. Yang, A deep learning approach to on-node sensor data analytics for mobile or wearable devices, IEEE Journal of Biomedical and Health Informatics 21 (2017) 56-64.

[20] C.A. Ronao, S. Cho, Human activity recognition with smartphone sensors using deep learning neural networks, Expert Syst. Appl. 59 (2016) 235-244.

[21] X. Zheng, J. Ordieres-Meré, Development of a Human Movement Monitoring System Based on Wearable Devices, (2014).

[22] X. Zheng, J. Ordieres-Mere, Detection and Analysis of Tremor Using a System Based on Smart Device and NoSQL Database, (2015), pp. 242-248.

[23] M. Abadi, A. Agarwal, P. Barham, E. Brevdo, Z. Chen, C. Citro, et al., Tensorflow: Large-scale Machine Learning on Heterogeneous Distributed Systems, (2016) arXiv preprint arXiv:1603.04467.

[24] Y.T.F. Tang, Learn: TensorFlow's High-level Module for Distributed Machine Learning, (2016) arXiv preprint arXiv:1612.04251.

[25] M. Divya, N.B. Biradar, IOTA-next generation block chain, International Journal of Engineering And Computer Science 7 (04) (2018) 23823-23826.

[26] S. Popov, The Tangle. Cit. on, (2016), p. 131.

[27] T. Sztyler, H. Stuckenschmidt, On-body Localization of Wearable Devices: an Investigation of Position-aware Activity Recognition, (2016), pp. 1-9.

[28] Y. LeCun, K. Kavukcuoglu, C. Farabet, Convolutional Networks and Applications in Vision, (2010), pp. 253-256.

[29] M. Zeng, L.T. Nguyen, B. Yu, O.J. Mengshoel, J. Zhu, P. Wu, et al., Convolutional Neural Networks for Human Activity Recognition Using mobile Sensors, (2014), pp. 197-205.

[30] J. Yang, M.N. Nguyen, P.P. San, X.L. Li, S. Krishnaswamy, Deep Convolutional Neural Networks on Multichannel Time Series for Human Activity Recognition, (2015).

[31] Y. Chen, Y. Xue, A Deep Learning Approach to Human Activity Recognition Based on Single Accelerometer, (2015), pp. 1488-1492. 


\subsection{Publication 5}

- Title: Discovering the patterns of energy consumption, GDP, and CO2 emissions in China using the cluster method

- Authors: Bing Gong, Xiaochen Zheng, QingGuo, Joaquín Ordieres-Meré

- Publication date: 2018/10/29

- Journal: Energy

Impact Factor: 4.968 (2017) / 5.582 (5 year)

Quartile: Q1

ISSN: $0360-5442$

Publisher: PERGAMON-ELSEVIER SCIENCE LTD, KIDLINGTON, OXFORD OX5 1GB, ENGLAND

- Volume: 166

- Pages: 1149-1167

- DOI: https://doi.org/10.1016/j.energy.2018.10.143

- Copyright statement: This study is published in subscription journals. The copyright of the postprint manuscript (accepted version after peer review) belongs to the publisher. According to the policy of the publisher, "Authors publishing in Elsevier journals have wide rights to use their works for teaching and scholarly purposes without needing to seek permission", including "inclusion in a thesis or dissertation' $55^{6}$ The final publication is available from the publisher https://doi.org/10.1089/tmj.2014.0102.

\footnotetext{
${ }^{5}$ https://www.elsevier.com/__data/assets/pdf_file/0007/55654/AuthorUserRights.pdf

${ }^{6}$ https://www.elsevier.com/about/policies/copyright/permissions
} 


\title{
Discovering the patterns of energy consumption, GDP, and $\mathrm{CO}_{2}$ emissions in China using the cluster method
}

\author{
Bing Gong ${ }^{b}$, Xiaochen Zheng ${ }^{\text {a, }}$, Qing Guo ${ }^{\text {c }}$, Joaquín Ordieres-Meré ${ }^{a}$ \\ ${ }^{a}$ PMQ research group, Industrial Engineering School (ETSII), Universidad Politécnica de Madrid, Calle de José Gutiérrez Abascal 2, 28006, Madrid, Spain \\ b Corning China, Manufacturing Technology \& Engineering Asia Department, Regional Headquarters Jinqiao office, 358 Lu Qian Road, Jinqiao Export \\ Processing Zone, Pudong New Area, Shanghai, 201206, China \\ ${ }^{c}$ School of Economics and Trade, Guangdong University of Foreign Studies, Guangzhou, 510420, China
}

\begin{abstract}
A B S T R A C T
This paper explores the effects of enforced Chinese policies regarding environmental matters at the provincial level. The paper proposes several features and explores datasets from the last four Five-Year Plans, which extensively covered the period from 1995 to 2015.

As the datasets are multidimensional, dimensional reduction is applied with a control of information loss described by data variance preservation. A data-based clustering algorithm is applied to identify the similarities, and characteristics are explored in relation to the specific features.

The use of this method makes it possible to identify the changes in the relevance of the features in the most recent Five-Year Plan, in which decarbonization is relevant instead of productivity. This method also makes it possible to identify specific efforts several provinces have made to introduce cleaner energy production.

Following the analysis, it is concluded that the proposed features enable the proposed method to be considered a tool for analyzing the real-world effects of the adopted policies.
\end{abstract}

(c) 2018 Elsevier Ltd. All rights reserved.

\section{Introduction}

$\mathrm{CO}_{2}$, one of the greenhouse gases, is the leading cause of global warming, rising sea levels, and frequent outbreaks of extreme weather. One of the main contributors to $\mathrm{CO}_{2}$ emissions is the burning of fossil fuels, which is the most common way to produce energy to maintain different aspects of human society, such as transportation, industry, agriculture, and commercial and residential buildings. The issues related to energy and pollution, as well as their impact on health and other areas, have been given considerable attention by citizens, governments, and academics.

As one of the world's largest energy consumers, China has surpassed the United States, becoming the largest $\mathrm{CO}_{2}$ emitter in the world and accounting for $25 \%$ of the world's carbon emissions. In recognition of this issue, the Chinese government has implemented a series of actions and regulations to deal with climate change. In

\footnotetext{
* Corresponding author.

E-mail addresses: gongbing1112@gmail.com (B. Gong), xiaochen.zheng@ alumnos.upm.es (X. Zheng), qingguovip@gmail.com (Q. Guo), j.ordieres@upm.es (J. Ordieres-Meré).
}

2016, it announced the 13th Five-Year Plans (FYPs) [16]. The plans explain the strategies of the Chinese central government related to all aspects of social and economic development. One of the most important aspects is its intention to reduce greenhouse gas emissions and improve air and water quality. At the 2009 Copenhagen Climate Summit, the Chinese government committed to cutting its carbon emissions by $40-45 \%$ per GDP unit from its 2005 levels by the year 2020 .

To achieve the emission reduction target, local pollution abatement should be implemented. As one of the largest countries in terms of landmass, China's patterns of economic activity, energy consumption, and population vary greatly among its provinces, sectors, and regions. Therefore, a comprehensive analysis of carbon emissions and other pollutants is required in order to propose a proper policy, such as an allocation of $\mathrm{CO}_{2}$ emission quotas by province, in order to achieve the national carbon emission reduction target.

Since anthropogenic $\mathrm{CO}_{2}$ emissions are highly related to socioeconomic activities, it is necessary to consider factors such as GDP and urbanization when analyzing $\mathrm{CO}_{2}$ emission patterns. In this way, dynamic changes in energy usage and $\mathrm{CO}_{2}$ emissions can be connected to these socio-economic activities. Such a concept is 
closely related to relevant policies, such as deep decarbonization at residential level one [4], among others. In addition, a comprehensive and dynamic indicators were designed combining with our proposed Principle Component Analysis (PCA) - Cluster methodology to provide an innovative assessment of policies and programs for energy issues in this research.

\section{Literature review}

A series of studies have analyzed the relationships between energy consumption, economic growth, and $\mathrm{CO}_{2}$ emissions. These analyses focused on different countries and areas, including European Union (EU) countries [2]; Brazil, the Russian Federation, India, and China (BRIC) [17]; the Middle East and North Africa (MENA) region [1]; and the Gulf Cooperation Council (GCC) countries [18]. For instance, the study by Ref. [18] examined the empirical relationships between economic growth, energy consumption, and $\mathrm{CO}_{2}$ emissions, and it calculated trends in the decoupling effect in GCC countries. Their study indicated that a positive and significant association exists between energy consumption and $\mathrm{CO}_{2}$ emissions and between economic growth and energy consumption both in the short and the long run, while no significant relationship was found between economic growth and $\mathrm{CO}_{2}$ emissions.

The authors of [20], on the other hand, compared the development of transportation with energy use, focusing on $\mathrm{CO}_{2}$ emissions in the EU15 countries. Three indicators related to energy and emissions were defined; these are immaterialization, dematerialization, and decarbonization. Immaterialization refers to the reduction of the energy intensity and transport intensity production, and it can be measured by the indicator energy intensity (Energy/GDP);Dematerialization is defined as the reduction in the carbon intensity of energy production and the carbon intensity of transport $\left(\mathrm{CO}_{2} /\right.$ Energy); Decarbonization is the reduction of carbon use among the entire economy, and it can be seen as a link between immaterialization and dematerialization. It is measured by the carbon intensity of the economy $\left(\mathbf{C O}_{2} / \mathbf{G D P}\right)$. This study grouped the EU15 countries based on each of the three previously mentioned indicators in order to establish different patterns. A limitation of this study is that it lacked a statistical analysis for identifying the main influential factors in each group.

Almost all studies have agreed that an accurate understanding about the relationships between energy consumption, economic growth, and $\mathrm{CO}_{2}$ emissions is a prerequisite for good energy and pollution policies. However, diverse patterns were found in different areas due to the variances in industrial and economic structures, making it impossible to offer any universal policy recommendations [11].

As one of the largest energy consumers and $\mathrm{CO}_{2}$ emitters in the world, China has attracted lots of research focusing on this topic. A study by Ref. [24] explored the regional characteristics of interprovincial $\mathrm{CO}_{2}$ emissions and the regional distribution of the reduction of the emission intensity in China. It used K-means, hierarchical clusters, and PSO-FCM clustering algorithms; the latter is a method that uses Particle Swarm Optimization (PSO) on Fuzzy-C means (FCM). To differentiate it from the previous traditional studies that divided China into regions (eastern, central, and western), the research by Ref. [24] examined how the regions correspond with the divisions by economic development level. In addition, five indices, including total emissions, per capital emissions, $\mathrm{CO}_{2}$ emissions per GDP (intensity of $\mathrm{CO}_{2}$ emissions), $\mathrm{CO}_{2}$ emissions per unit of energy, and the proportion of industrial $\mathrm{CO}_{2}$ emissions, are used to describe $\mathrm{CO}_{2}$ emissions in China.

The research by Ref. [19] analyzed China's city-level $\mathrm{CO}_{2}$ emissions due to energy consumption by Defense Meteorological Satellite Program/Operational Line-scan System (DMSP/OLS) nighttime light imageries and explored the major driving forces for feasible mitigation policies. It included a recommendation to reduce the growth rate of $\mathrm{CO}_{2}$ consumption without compromising economic development by optimizing the industrial structure of technology-intensive areas and improving energy efficiency in other areas.

Based on the log mean Divisia index (LMDI), the study by Ref. [21] provided a new way of analyzing changes pertaining to $\mathrm{CO}_{2}$ emission-GDP (C-G) decoupling indicators in China. The changes in $\mathrm{C}-\mathrm{G}$ indicators are decomposed into the $\left(\mathrm{CO}_{2}\right.$ emissionfossil energy consumption (C-EF) decoupling indicator effect, the fossil energy consumption-total energy consumption (EF-E) decoupling indicator effect, and the total energy consumption-GDP (E-G) decoupling indicator effect. The study found that the E-G decoupling effect plays an important role in the changes of the $C-G$ decoupling indicator. However, the C-EF decoupling effect played a minor role in the changes of the $\mathrm{C}-\mathrm{G}$ decoupling indicator from 1996 to 2013.

A special IPAT $($ Impact $=$ Population $\times$ Affluence $\times$ Technology $)$ identification case that reveals the importance of distinct components or factors in the historical data of energy-related $\mathrm{CO}_{2}$ emissions, which is often called the Kaya identity, has been widely utilized in many studies [6,25]. Previous research [25] separated urban emissions into four components (emissions from primary, secondary, and tertiary industries and residential areas) to determine the interactions of 13 macro influential factors. Thirty Chinese provinces were classified on the basis of historical data for these macro factors. Shapley value decomposition was adopted to identify the key factors; in light of this, the related policy control was promoted.

These studies are beneficial for understanding the relationships between energy consumption, economic growth, and $\mathrm{CO}_{2}$ emissions in China. However, there is a level of uncertainty that cannot be ignored regarding official figures. The reason for this is that China reported a reduction in its coal consumption of $2.9 \%$ in 2015 [15]. This was hailed as historic after China's meteoric growth in the 2000s. Similar preliminary coal consumption statistics are provided every year, and will be watched closely after China's recent slowdown in emissions growth and its pledge to peak emissions by or before 2030. However, Chinese energy statistics are frequently revised and often contain large anomalies [12,13], which imply high uncertainty levels. For example, British Petroleum used different Chinese data sources to estimate a $0.9 \%$ increase in $\mathrm{CO}_{2}$ emissions in 2014 [3,23].

In the last two decades, the data envelopment analysis (DEA) analytical framework has been widely applied in the environmental and energy fields. In particular [7], used its dynamic variant to evaluate the energy efficiency of OECD (Organisation for Economic Cooperation and Development) countries and China.

The main limitations of the existing studies are their lack of dynamic views in revealing the changes in China's energy usage and $\mathrm{CO}_{2}$ emissions at the provincial level, as well as their failure to use proper techniques for identifying the most important factors regarding different dynamic indicators. To address these deficiencies, this study seeks to dynamically analyze the patterns of energy usage and $\mathrm{CO}_{2}$ emissions at the provincial level by utilizing the indicators related to immaterialization, dematerializaion, decarbonization, and population by adopting several analytical techniques. In addition, a statistical analysis of potential influential factors will be undertaken to determine the main factors of each category based on the modeling result. The outcome of the results can help us to understand the driving factors and benefits of the policymakers, gaining closer views of specific provinces to promote practicable policies. 


\section{Methodology}

\subsection{Study area}

This study focuses on 30 provinces in China. Fig. 1 indicates the locations of these provinces. However, this map does not include the two special administrative regions of Hong Kong and Macau because their areas are too small. The map also excludes the disputed territory of Taiwan. Table 1 shows the names of the provinces and their corresponding abbreviations. $\mathrm{BJ}, \mathrm{SH}, \mathrm{TJ}$, and $\mathrm{CQ}$ are the four most prominent metropolitan areas, with populations that exceed ten million. This study treats these four cities as provinces, as they have provincial statuses in China based on Chinese political and administrative systems. All of the data were obtained from the Chinese yearbook. This data includes the energy usage of each type and each province and the GDP and urbanization for each province. This study focuses on the 9th, 10th,11th, and 12th FYPs, which occurred from 1995 to 2000, 2001 to 2005, 2006 to 2010 , and 2011 to 2015, respectively.

\subsection{Methods}

The strategy of this research study consists of the following five parts, as demonstrated in Fig. 2: the scope of this study, the key factors generated from the scope, the datasets for the data analysis, the utilized methods aimed toward exploring the carbon emission patterns across China's provinces, and lastly, the policy recommendations.

In this study, we focus on the scope of the relationships between energy, carbon emissions, and economic and social factors. The key features derived from these four aspects are immaterialization, dematerialization, decarbonization, energy-density, and social productivity. The details are described in Section 3.3.

The data analysis will be launched by different periods based on the FYPs. This is because in the first year of every FYP, the central government explains the strategy and pinpoints the targets regarding economic and social development. The analytic datasets, therefore, could be consistent with FYPs in order to deeply understand the correlations between the changes of the policies and the variances of the features.

The datasets are fed into the methods-dimensionality reduction and cluster algorithms for further data analysis. The goals of the analysis are to find the key features of the most significant
Table 1

The names of the provinces and their abbreviations.

\begin{tabular}{llll}
\hline Area Name & Abbre. & Area Name & Abbre \\
\hline Bei Jing & BJ & He Nan & HN \\
Tian Jin & TJ & Hu Bei & HuB \\
He Bei & HeB & Hu Nan & HuN \\
Shan Xi & ShanX & Guang Dong & GD \\
Inner Mongolia & IM & Guang Xi & GX \\
Liao Ning & LN & Hai Nan & HaiN \\
Ji Lin & JL & Chong Qing & CQ \\
Hei Long Jiang & HLJ & Si Chuan & SC \\
ShangHai & SH & Gui Zhou & GZ \\
Jiang Su & JS & Yu Nan & YN \\
Zhe Jiang & ZJ & ShannXi & ShannX \\
An Hui & AH & GanSu & GS \\
Fu Jian & FJ & QingHai & QH \\
Jiang Xi & JX & NingXia & NX \\
Shan Dong & SD & XinJiang & XJ \\
\hline
\end{tabular}

variances among the provinces, and to group the provinces by all the features to profoundly understand and explore the patterns of energy consumption, GDP, and $\mathrm{CO}_{2}$. emissions.

\subsection{Data sources and feature selection}

Due to the lack of $\mathrm{CO}_{2}$ emission statistics in China, $\mathrm{CO}_{2}$ volume will be calculated by the types of energy and the corresponding $\mathrm{CO}_{2}$ emission factors that are provided by the Intergovernmental Panel on Climate Change (IPCC). This was done by most of the existing studies [19]. The energy consumption that was considered includes conservation sectors (heating supply and thermal power industries) and final consumption sectors (farming, forestry and animal husbandry; fisheries \& water conservancy; construction; transport, storage and postal; wholesale and retail trade; hotels and restaurants; residential consumption; and other sectors).

$\left(\mathrm{CO}_{2}\right)_{j}=\frac{44}{12} \sum_{i=1}^{n} K_{i} E_{i}$

where $E_{i}$ is the energy consumption per energy type $i$, and $K_{i}$ is the conversion factor $K$ for energy type $i$. According to [24], $K$ is defined at the provincial level as:

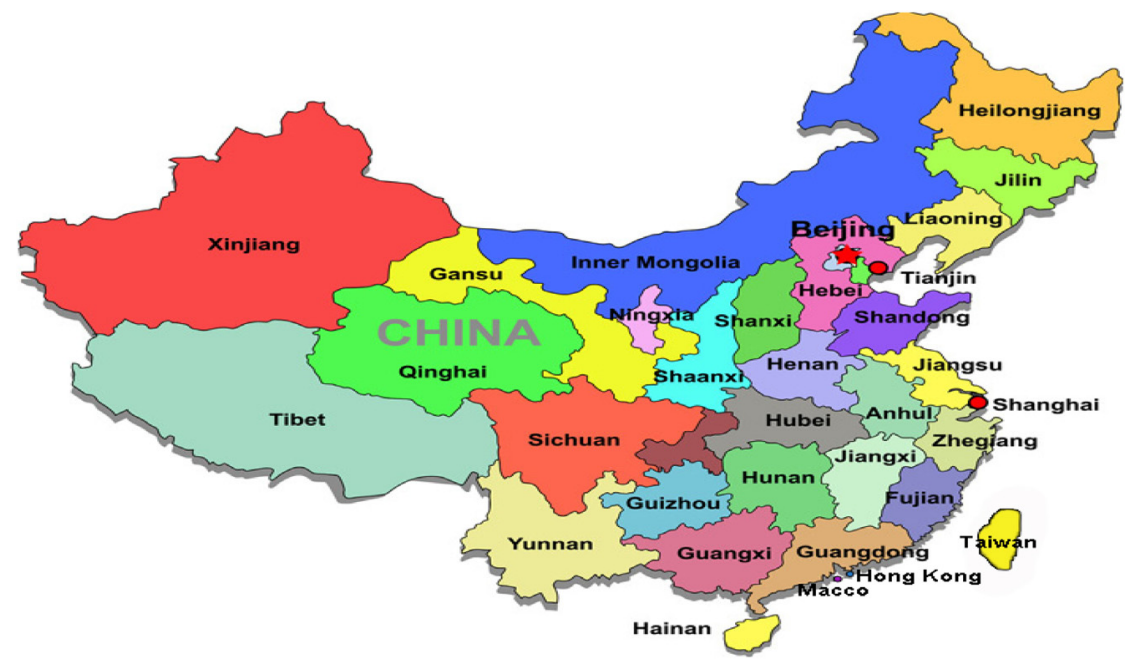

Fig. 1. A map of the provincial level administrative divisions of China. 


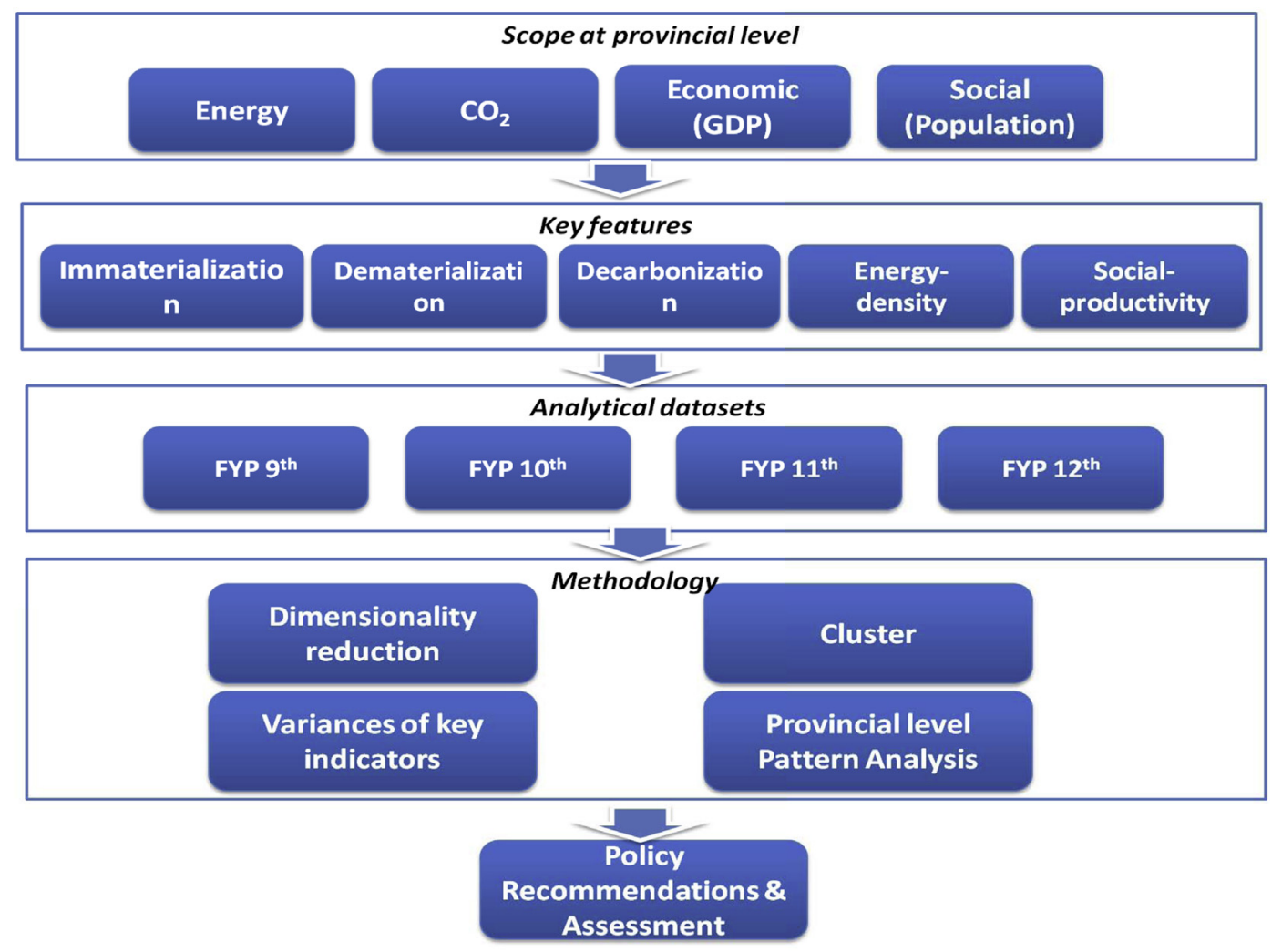

Fig. 2. Flowchart of the methodology.

$K_{i}=\left(e_{i} \times c_{i} \times 10^{-3}\right) \times O_{i}$

where $e_{i}$ is the net calorific value of the the of fuel, and $c_{i}$ represents its carbon content. Finally, $\mathrm{O}_{i}$ is the carbon oxidation rate of the $i$ th fuel.

Details of the types of energy and conversion factors to be considered are presented in Table 2. The calculation of $\mathrm{CO}_{2}$ volume per province is then implemented by Equations (1) and (2). The units of the parameters mentioned in the equations are listed in Table 3.

After obtaining the $\mathrm{CO}_{2}$ emissions and energy consumption data, the energy consumption of each province can be aggregated by the following equation (3).

$E_{p}=\sum_{i=1}^{n} E_{i}$

Using the GDP, energy consumption, and $\mathrm{CO}_{2}$ emissions data and inspired by Ref. [20], we modified the immaterialization, dematerialization, and decarbonization definitions slightly by simply reversing their initial definitions. Because they highlight variations, we adopted the $\mathrm{CO}_{2}$ emission variations to be the common references. The established features were calculated for each province by the 4 to 6 equations.

immaterialization $^{*}=\frac{G D P_{y 1}}{E_{y 1}}-\frac{G D P_{y 2}}{E_{y 2}}$

dematerialization $^{*}=\frac{E_{y 1}}{\left(\mathrm{CO}_{2}\right)_{y 1}}-\frac{E_{y 2}}{\left(\mathrm{CO}_{2}\right)_{y 2}}$

decarbonization $*=\frac{G D P_{y 1}}{\left(C O_{2}\right)_{y 1}}-\frac{G D P_{y 2}}{\left(C O_{2}\right)_{y 2}}$

In addition to these features, it is helpful to consider the influence of Pop (population figures) [22]. Therefore, we additionally adopted the following features:

Table 2

$\mathrm{CO}_{2}$ Conversion factors for each type of energy (ton/tce).

\begin{tabular}{lllll}
\hline Items & Raw Coal & Cleaned Coal & Other Washed Coal & Briquettes \\
Coefficient & 0.485 & 0.611 & 0.355 & 0.424 \\
\hline Items & Coke & Coke Oven Gas & Other Gas & Crude Oil \\
Coefficient & 0.746 & 0.196 & 0.092 & 0.819 \\
\hline Items & Kerosene & Diesel Oil & Gasoline & 0.798 \\
Coefficient & 0.824 & 0.845 & 0.864 & LPG Oil \\
\hline Items & Refinery Gas & Natural Gas & Other Petroleum Products & 0.846 \\
Coefficient & 0.709 & 0.589 & 0.788 & 0.856 \\
\hline
\end{tabular}


Table 3

Nomenclature.

\begin{tabular}{|c|c|c|}
\hline & Description & Unit \\
\hline GDP & Gross Domestic Product & RMB (in billion) \\
\hline $\mathrm{CO}_{2}$ & Carbon dioxide & ton \\
\hline$i$ & Energy type $i$ & - \\
\hline E & Energy consumption & $\begin{array}{l}\text { metric tons of coal equivalent } \\
\text { (tce) }\end{array}$ \\
\hline $\mathrm{K}_{i}$ & $\begin{array}{l}\mathrm{CO}_{2} \text { conversion factor for energy } \\
\text { type } i\end{array}$ & ton/tec \\
\hline $\mathrm{p}$ & Province & - \\
\hline Pop & Population & 10000 persons \\
\hline $\mathrm{y}$ & Year & - \\
\hline$\triangle(\mathrm{GDP} / \mathrm{E})$ & Immateriaization $^{*}$ (Equation (4)) & Billion/tce \\
\hline$\triangle\left(\mathrm{E} / \mathrm{CO}_{2}\right)$ & Dematerialization*(Equation (5)) & tce/ton \\
\hline $\begin{array}{c}\triangle(\mathrm{GDP} / \\
\left.\mathrm{CO}_{2}\right)\end{array}$ & Decarbonization $^{*}$ (Equation (6)) & Billion/ton \\
\hline$\triangle(\mathrm{E} / \mathrm{Pop})$ & Energy density (Equation (7)) & tce/(10000 persons \\
\hline $\begin{array}{c}\triangle(\mathrm{GDP} / \\
\text { Pop })\end{array}$ & Social-productivity & 100 thousands RMB/person \\
\hline
\end{tabular}

energy_density $=\frac{E_{y 1}}{P o p_{y 1}}-\frac{E_{y 2}}{P o p_{y 2}}$

social_productivity $=\frac{G D P_{y 1}}{P_{0 p_{y 1}}}-\frac{G D P_{y 2}}{P_{0 p_{y 2}}}$

All of these features were calculated by province as well as by the official economic planning period of analysis.

\subsection{Data analysis and techniques}

As the goal of the paper is to analyze the behavior of the provinces by means of different FYPs, the appropriate segmentation of data will bring about multidimensional datasets. The datasets from the different FYPs as inputs are fed into the dimensional reduction algorithm and cluster algorithms in order to analyze the main contributors from the features and the provincial patterns from the same cluster. Specifically, it will be useful to reduce the dimension of the data to allow for a low dimensional representation of the data. Dimensionality reduction can also be seen as the process of deriving a set of degrees of freedom, which can be used to reproduce most of the variability in a dataset. This means that a dataset produces a compact low-dimensional encoding of the given highdimensional dataset.

Dimensionality reduction has been used in many disciplines [27]. Projection transforms the data from the multidimensional space into a space of fewer dimensions. The data transformation may be linear, as in principal component analysis (PCA) or linear discriminant analysis, but there are also many nonlinear dimensionality reduction techniques. Well known techniques include Kernel PCA, Generalized discriminant analysis, Isomap and Locally linear embedding.

The second step of the process is to look for similarities and cluster such datasets. The reason for clustering is to partition the data into a specific number of clusters that maximize intra-cluster similarities and minimize inter-cluster similarities. However, none of the cluster algorithms can select the optimal number of groups (clusters) automatically. The problem of optimal clustering is to find a cluster configuration that is optimized in light of a certain evaluation criterion. Different decision criteria, from the squared error sum of the hierarchical clustering algorithm to the Bayes information criteria for a mixture of Gaussian models ([9]), are available.

Traditional cluster algorithms can be divided into partitioning methods, hierarchical methods, density-based methods, grid-based methods, and model-based methods. This study uses hierarchical (agglomerative) methods and model-based methods (Gaussian Mixture Models [GMMs]) for further analysis.

The method proposed here was inspired by previous works $([5,10])$ and uses dimensional reduction with an interpretation of the relevant eigenvectors and similar clustering behaviors.

\subsubsection{Principal component analysis}

PCA is a classical analysis method that provides a sequence of best linear approximations to a given high-dimensional observation dataset. Although it is one of the most popular techniques for reducing dimensionality, its effectiveness is limited by its global linearity. Because it provides information about the amount of variability captured, it is adopted in this paper, regarding the losses, when convenient. Given a set of data of $n$ dimensions, PCA seeks to find a linear subspace of dimension $d$ lower than $n$ such that the data points lie mainly on this linear subspace. Such a reduced subspace (manifold) attempts to maintain most of the data's variability.

The linear subspace can be specified by $d$ orthogonal vectors that form a new coordinate system; these are called the "principal components". Principal components are orthogonal, linear transformations of the original data points; thus, there can be no more than $n$ of them. One advantage of this technique is that the quality of the projection can be determined as the percentage of the variance in the dataset retained in the projection. Therefore, an analyst can decide the quality he or she wants to adopt.

The classical definition of PCA can be found in Ref. [8].

\subsubsection{Gaussian Mixture Models}

GMMs are often utilized for data clustering. GMMs consist of $\mathrm{K}$ components (K Gaussian Distribution $N\left(x \mid \mu_{k}, \sum_{k}\right)$. The probability density function can be represented by Equation (9).

$\left.p(x)=\sum_{k=1}^{k} p(k) p(x \mid k)=\sum_{k=1}^{k} \pi_{k} N \quad x \mid \mu_{k}, \sum_{k}\right)$

The parameters of GMM include $\pi_{k}, \mu_{k}$, and $\sum_{k}$. The idea is to find a combination of the parameters for achieving the highest probability with the data points $\operatorname{Max}\left(\Pi_{i=1}^{N} p\left(x_{i}\right)\right)$, where $N$ is the number of given data points and $p_{x}$ is the density of the probability distribution for all the data points. $\prod_{i=1}^{N} p\left(x_{i}\right)$ is also called the likelihood function. In order to maximize the likelihood function, expectationmaximization is employed.

$N(x)=|2 \pi \Sigma|^{-\frac{1}{2}} \exp \left\{-\frac{1}{2}(x-\mu)^{t} \Sigma^{-1}(x-\mu)\right\}$

Equation (10) shows the multivariate normal distribution. $\Sigma^{-1}$ is the covariance matrix that determines the shapes of the clusters. There is a trade-off between the shape of the clusters and the number of clusters. If the shape of the clusters is simple, more clusters should be used for better clustering, and vice versa.

Deciding on the number of clusters is essential for the rest of the working scenario and testing by other external data [20]. In this study, the optimal number of clusters is defined using the Bayesian information criterion $(B I C)$ for the expectation-maximization (EM) method. $B I C=-2 \log (L)+\operatorname{mlog}(n)$, where $L$ is the likelihood function and $m$ is the number of free parameters to be estimated. Although a model with a low BIC is superior to one with a high BIC because of the former's lower complexity, when clustering procedures are considered, BIC values are an approximation for 
integrated (not maximum) likelihoods, and the model with the greatest integrated likelihood (Bayes factor) is generally preferred. Therefore, the recommendation is to choose the model with the largest BIC.

\subsubsection{Agglomerative hierarchical clustering}

The hierarchical cluster algorithm is a cluster analysis method that appears to build a hierarchy of clusters. Based on this strategy, algorithms fall into two types: agglomerative and divisive. The agglomerative strategy is a "bottom-up" approach that starts its own cluster for each sample. Then, the pairs of clusters are merged as one moves up the hierarchy. The divisive strategy refers to a "top-down" approach; that is, an approach in which all the samples belong to one cluster and afterwards are split recursively when one moves down the hierarchy. This study will use the agglomerative hierarchical clustering approach.

Metric and linkage criteria are the two determinants of the clustering results. Metric is the measurement of the distance between the pairs of observation. Metric also directly determines the shape of the clustering. Several metrics are widely used in hierarchical clustering algorithms. These include Euclidean distance, Square Euclidean distance, and Manhattan distance. Linkage criteria, which are the clustering rules used to determine the distance between two sets of observations, usually include maximum or complete linkage clustering, minimum or single-linkage clustering, average linkage clustering, and centroid linkage clustering methods.

This work will use Euclidean distance and centroid linkage clustering, as presented in Equations (11) and (12), for the hierarchical clustering algorithm.

$\|a-b\|=\sqrt{\sum_{i=1}^{n}\left(a_{i}-b_{i}\right)}$

$\left\|c_{s}-c_{t}\right\|$ where $c_{s}$ and $c_{t}$ are the centroids of clusters $s$ and $t$, respectively.

\section{Results}

\subsection{Description analysis}

Fig. 3 shows the 15 provinces that had the highest $\mathrm{CO}_{2}$ emission volumes in 1995, 2001, 2005, 2011, and 2015. In 1995, the volume of $\mathrm{CO}_{2}$ emissions for all the provinces was less than 250 million tonnes. $\mathrm{HeB}$, JS, $\mathrm{LN}$, and SD had the highest $\mathrm{CO}_{2}$ emissions. From 1995 to $2000, \mathrm{CO}_{2}$ emissions did not significantly increase. In fact, some provinces (such as $\mathrm{HN}$, JL, and SC), experienced a decrease in their $\mathrm{CO}_{2}$ emissions. The most notable increase in $\mathrm{CO}_{2}$ emissions began in 2001. $\mathrm{CO}_{2}$ emissions rose in some areas, including $\mathrm{HeB}$, HuN, JS, and SD. In SD Province, emissions more than doubled to 612.5 million tonnes in 2005 compared to 250 million tonnes in 2000, and grew to more than 840 million tonnes in 2010. We can determine from the figures that such a high rate of growth from 2001 to 2010 was somehow contained until 2015, when similar figures were shown.

Fig. 4 shows the GDP values for the top $\mathrm{CO}_{2}$-emitting provinces in China, which are GD, HeB, HuN, IM, JS, LN, SD, and ShanX. GD is an area whose GDP increased notably during this period, from 800 billion RMB in 1995 to about 8000 billion in 2015. The economy of IM grew more slowly than that of the other sevenareas and was similar to that of ShanX, even when it had the highest $\mathrm{CO}_{2}$ emissions. It can be seen that the economies of the eight provinces aforementionedgrew slowly before 2000 , but much more quickly after 2000. Figs. 3 and 4 both show that the province with the highest amount of pollution in China (IM) might not be the most developed one. Meanwhile, provinces such as ShanX could have a similar GDP but a relatively low level of $\mathrm{CO}_{2}$ pollution. It is also useful to observe the behavior of LN Province. Its GDP figures for the most recent period were reviewed by the Chinese government 12 , which emphasizes the level of uncertainty that was previously discussed in the Literature Review section regarding its impact on the overall figures.

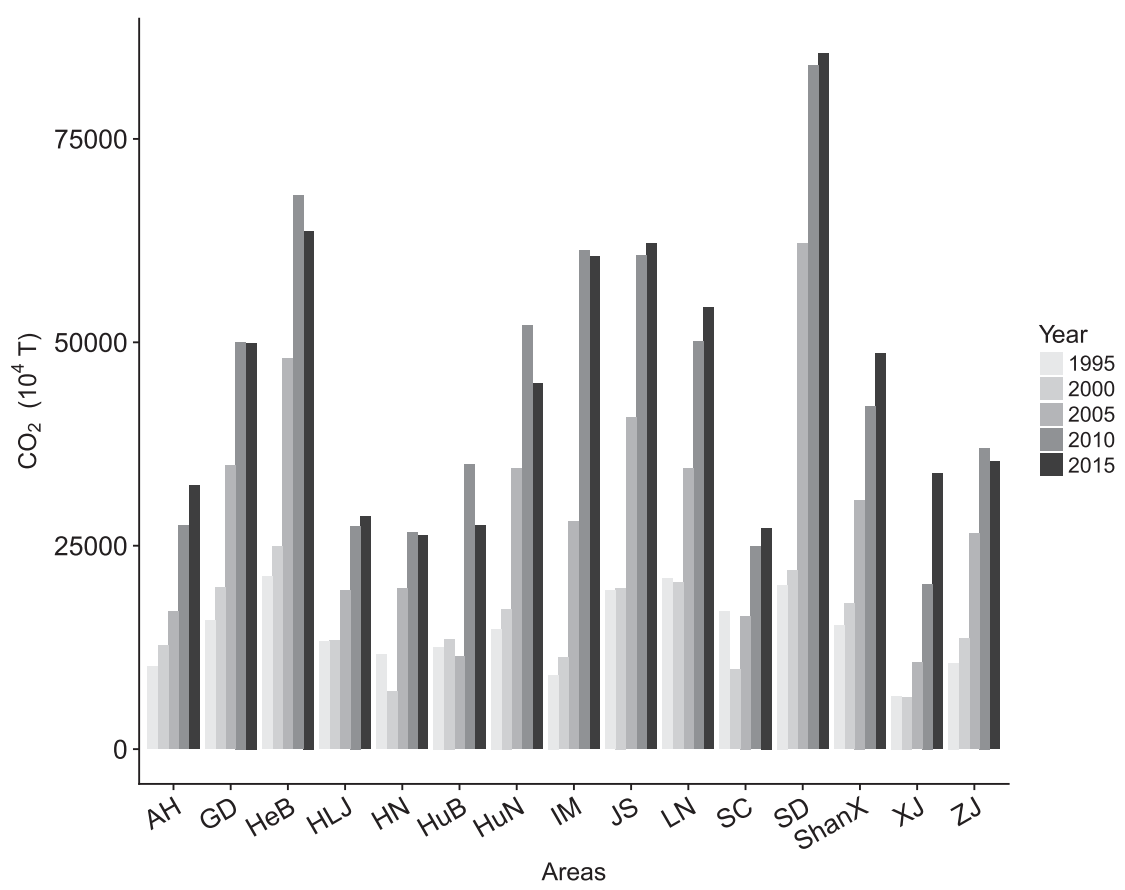

Fig. 3. The 15 provinces with the highest $\mathrm{CO}_{2}$ emission levels in China in 1995, 2000, 2005, 2010, and 2015 


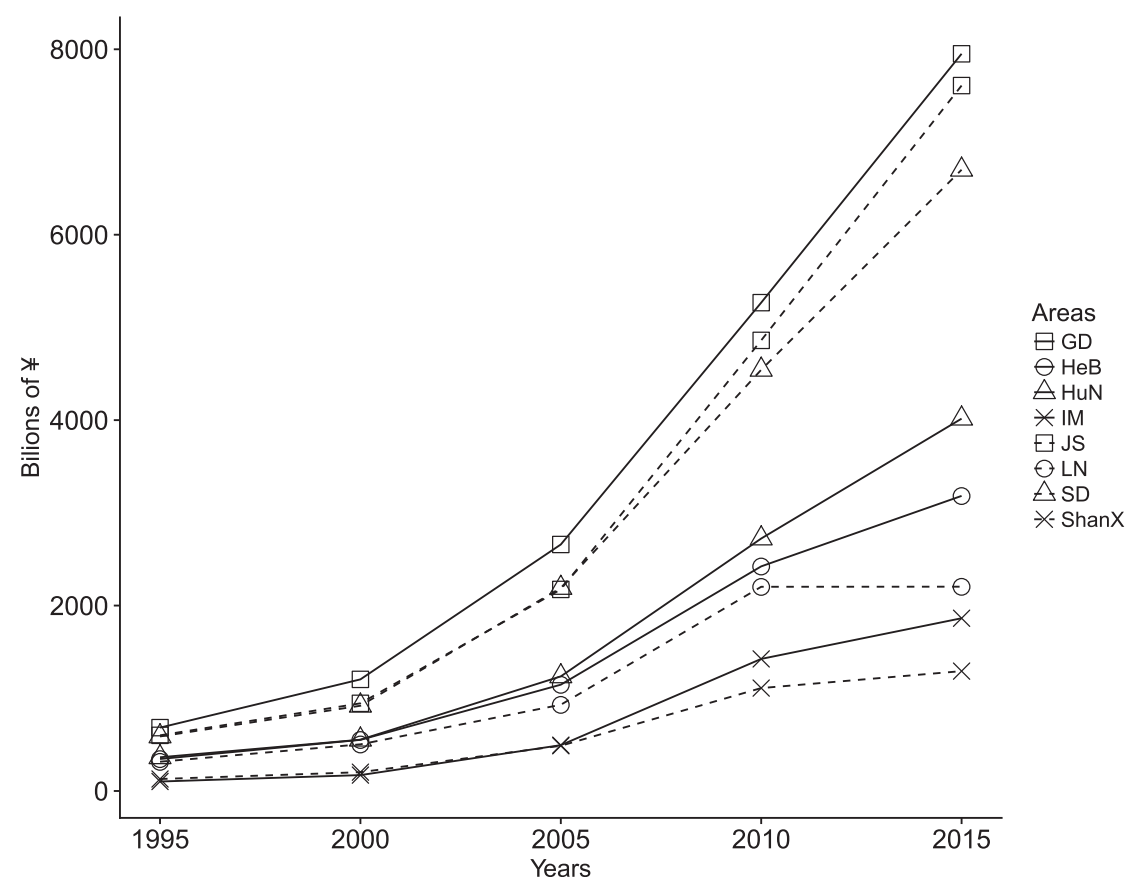

Fig. 4. GDPs of the Chinese provinces that had the highest levels of $\mathrm{CO}_{2}$ emissions from 1995 to 2015 .

Fig. 5 shows where the energy in China was used up to the year 2012. Raw coal (66\%) was still the main energy source in China until 2012 , followed by clean coal and crude oil, which accounted for $9 \%$ and $7 \%$ of the total energy consumption, respectively. This clearly shows that clean energy was not widely used before 2013.

\subsection{PCA dimensional reduction}

The dataset has been segmented according to FYP. The PCA method was then applied in accordance to the identified clusters, as seen in Fig. 6. The representativeness of the projection is described by Table 4 . The latter shows that the first two components explain more than $90 \%$ of the variance in the data for all the FYPs.

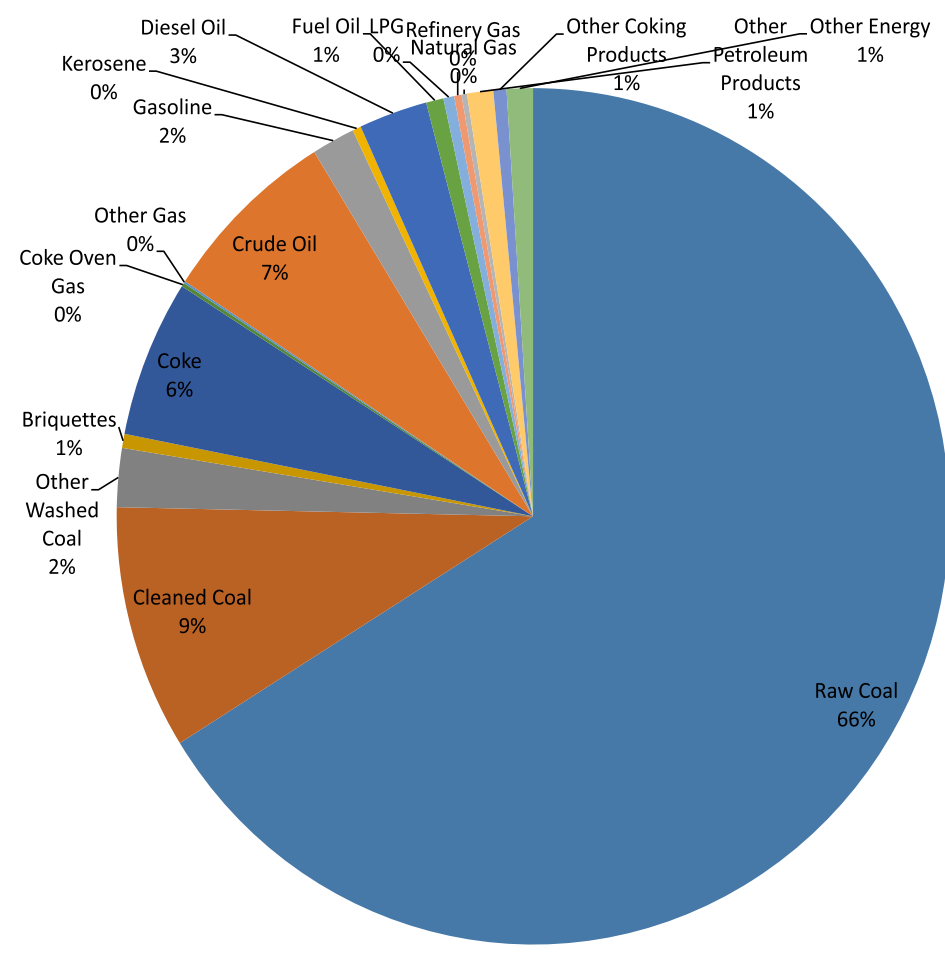

Fig. 5. China's energy consumption structure in 2012. 


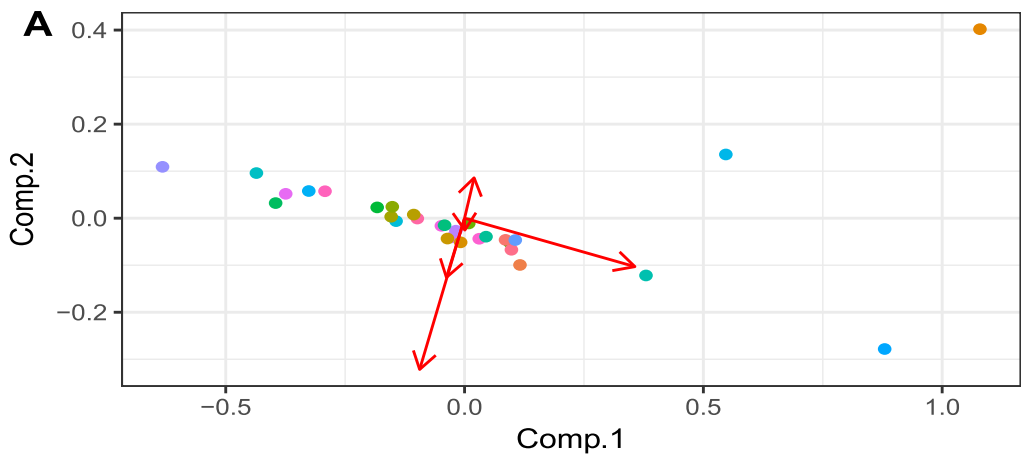

Province

- $\mathrm{AH}$

- $B J$

- $C Q$

- FJ

B

- GD

- GS

- $\mathrm{GX}$

- $\mathrm{GZ}$

- HaiN

- $\mathrm{HeB}$

- HLJ

- $\mathrm{HN}$

- HuB

- HuN

- IM

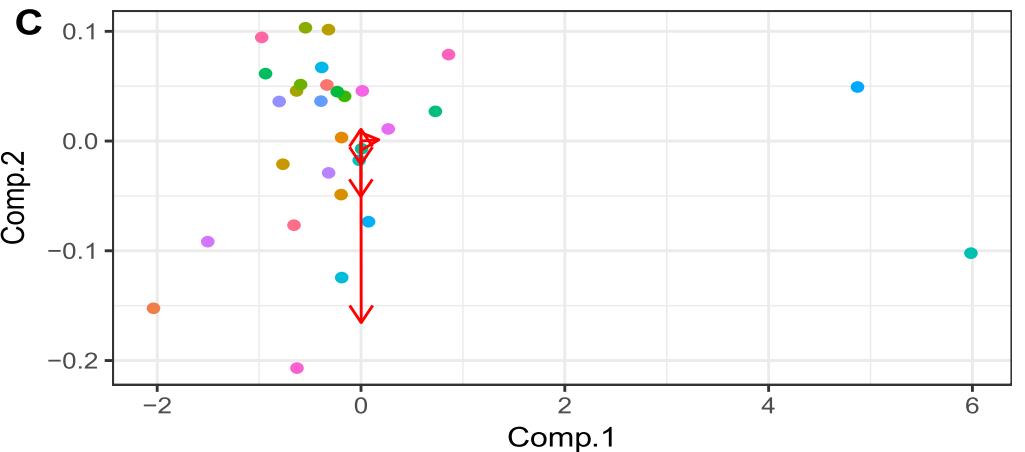

- JL

- JS

- JX

- LN

- NX

- $\mathrm{QH}$

- $S C$

- SD

- $\mathrm{SH}$

- ShannX

- ShanX

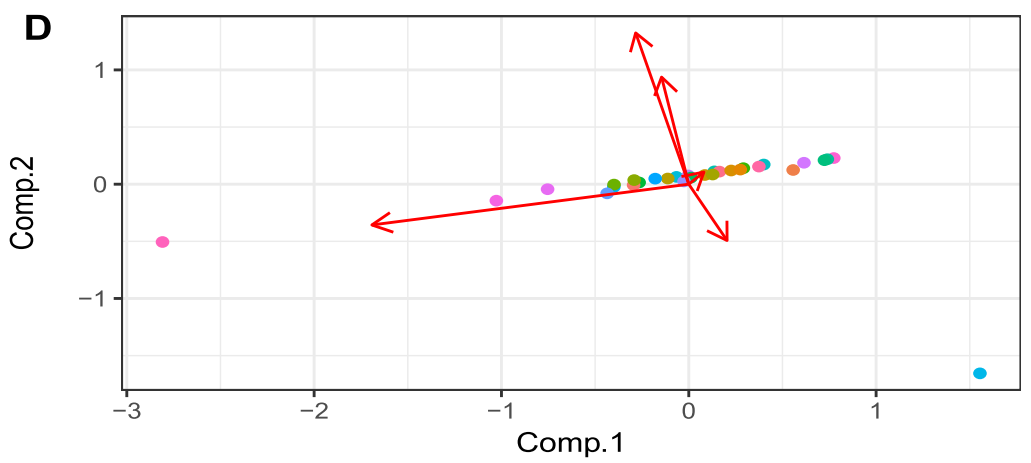

- TJ

- XJ

- YN

- ZJ

Fig. 6. $P C A$ projections. $A=1996-2000, B=2001-2005, C=2006-2010, D=2011-2015$.

Table 4

Percentage of explained variance by projected dimensions and total variance retained by the first two components.

\begin{tabular}{lllllll}
\hline FYP & $1^{\text {st }}$ Dim. & $2^{\text {nd }}$ Dim. & $3^{\text {rd }}$ Dim. & $4^{\text {th }}$ Dim. & $5^{\text {th }}$ Dim. & Tot. Var. \\
\hline $1996-2000$ & 69.5 & 21.1 & 5.3 & 3.8 & 0.3 & 90.6 \\
$2001-2005$ & 89.3 & 6.2 & 2.5 & 1.9 & 0.1 & 95.5 \\
$2006-2010$ & 96.8 & 1.9 & 0.9 & 0.4 & 0.05 & 98.6 \\
$2011-2015$ & 83.7 & 11.0 & 3.5 & 1.5 & 0.3 & 94.7 \\
\hline
\end{tabular}

The effective meaning of this information is that the structure of all the data in its original dimension is essentially less than the $10 \%$ differenced from its two-dimensional projection. Such difference is measured as the summation of the distances between all the pairs of data points over the number of points. (see Ref. [8]).

Therefore, we can use the two-dimensional projection as a valid representation of the different data subsets, as per the FYPs. The main interpretation of the two-dimensional projected data is that it highlights the very different behaviors of different provinces across 
different plans. Such distribution of projected points can help in understanding to what end the policies were properly implemented by actions, or if additional effects needed to be considered to explain these policies.

\subsection{Cluster analysis}

According to model-based clustering, classification, and estimating density based on finite normal mixture modeling, we will proceed to estimate the most suitable set of clusters per FYP according to the features that were identified in Section 3.3, but after being dimensionally reduced in order to avoid dimensionality issues by the year 2012. Therefore, a BIC will be used to identify the most suitable clustering strategy. This will provide the closest similarities between the cluster members and the largest dissimilarities between the clusters, as the goal is to better identify closely integrated behaviors by province in FYPs, according to the executions of the plans.

The data corresponding to these periods were evaluated by GMMs, with different hypotheses related to the following clusters:

- EEI: clusters with diagonal, equal volume and shape;

- EII: clusters with spherical, equal volume;

- EEV: clusters with ellipsoidal, equal volume and equal shape;

- EVE: clusters with ellipsoidal, equal volume and orientation; VII: clusters with spherical, unequal volume; and

- VEE: clusters with ellipsoidal, equal shape and orientation.

Different clusters were tested to determine the number of suitable clusters.

In China, the central government usually makes the major policies, and the local or city government is responsible for implementing them. Therefore, it is important to understand the characteristics of provinces in the same cluster based on their relevant features in order to design effective policies for reducing emissions.

\subsubsection{Period of 1996-2000}

The described methodology was used for this first period. It was discovered that most variance in former times was explained by energy density (see equation (7)) and immaterialization (see equation (4)), which are largely responsible for the first two eigenvectors. These features are relevant, as the measurements that discriminate the industrial structures are the investments in each type of industry and the usage patterns of various types of energy ([20]).

According to BIC (Fig. 7a), the best model-based cluster is found in model EEV with six components (clusters). In Fig. 7b, the clusters are shown according to the proposal from the Clustering Large Applications (CLARA) algorithm.

Based on these clusters, the BJ, TJ, HeB, IM, ZJ, AH, FJ, HuN, HaiN, and $\mathrm{QH}$ provinces are grouped together, as they have slightly increased the required energy in the reference period over their population increments. This effect occurred with some losses in GDP per unit of energy. We can consider this the reference behavior.

The second cluster includes ShanX, HLJ, SH, JS, SD, HuB, GD, GX, $\mathrm{GZ}, \mathrm{YN}$, and GS, which have similar tendencies to cluster 1 regarding immaterialization but with little improvement in energy density performance, as their energy variations increase less than their populations. We can consider this behavior to be of limited but harmonious growth.

The third cluster involves LN, JL, HN, SC, ShanX, and XJ, in which energy density figures decrease and immaterialization also increased. These can be considered the best results observed.
The main clusters have three outliers, which are:

- JX, which has acceptable immaterialization values but poor energy density evolution, with increases in variations of energy per population ratio,

- $\mathrm{CQ}$ which has extremely good immaterialization figures. However, they were obtained by an unbalanced energy management (huge energy variations over variations in population). It is typical of industrial development based on unclean energies,

- NX, which demonstrates the worst behavior, as it had losses in immaterialization and energy density. This means that even when it grew in relative energy over the population figures, the relative GDP over population fell slightly.

In this period, features such as dematerialization and decarbonization were responsible for less than $10 \%$ of the total variations in the dataset, which revealed no structural transformations of such features.

\subsubsection{Period of 2001-2005}

The results for this period showed that, in agreement with the previous one, most of the variances could be explained by energy density (see equation (7)). However, immaterialization is replaced by productivity (see equation (8)). Both features are largely responsible for the first two eigenvectors.

The BIC criteria (Fig. 8a) shows that the best model-based cluster is the EEVhypothesis with four components (clusters). The clusters are shown in Fig. 8b according to the proposal in the CLARA algorithm.

Based on these clusters, the BJ, SH, AH, JX, HuB, GX, HaiN, CQ, SC, and $\mathrm{QH}$ provinces are grouped together and have the highest $\Delta$ (Energy/Pop), where HuB has the worst behavior and $\Delta$ (Energy/ Pop) is positive.

The second cluster, which includes provinces such as TJ, HeB, LN, $\mathrm{JL}, \mathrm{JS}, \mathrm{ZJ}, \mathrm{HuN}$, and GZ, is characterized by slight decreases in $\Delta$ (Energy/Pop). The problem becomes the $\Delta$ (GDP/Pop), as the variations were not too significant. This behavior, although not optimal, can actively promote efficient energy usage.

Although all the clusters showed discrete figures for GDP variations, the main driving factor was the energy density. Such an evolution could be perceived in the third cluster, which contains provinces such as ShanX, IM, SD, and NX. The behavior that is seen in this cluster is an active reduction of the energy usage in relation to the increase of the population.

Finally, the fourth cluster includes HLJ, FJ, HN, GD, YN, ShanX, GS, and XJ. They show a moderate increment in energy usage over the increments of the population.

\subsubsection{Period of 2006-2010}

The results show that, in agreement with the previous period, most of the variances could be explained by the energy density (see equation (7)) and productivity (see equation (8)). Both features were again largely responsible for the first two eigenvectors.

According to BIC criteria (Fig. 9a), the best model-based cluster is the VEE hypothesis with two clusters. The clusters are shown in Fig. 9b, according to the CLARA algorithm proposal.

The fact that the best adjustment occurs with clusters of variable volumes indicates that there were unexpected behaviors in the dataset. Fig. 9b indicates significant increases of energy in NX, which can be considered a specific investment in the energy generation for those two provinces. Such an influence hides the significant improvement exhibited by other provinces such as $\mathrm{BJ}, \mathrm{SH}$, $\mathrm{HN}$, and YN. These provinces demonstrated excellent performances by showing $\Delta$ (Energy $/$ Pop $)<0$. However, a common aspect is that 


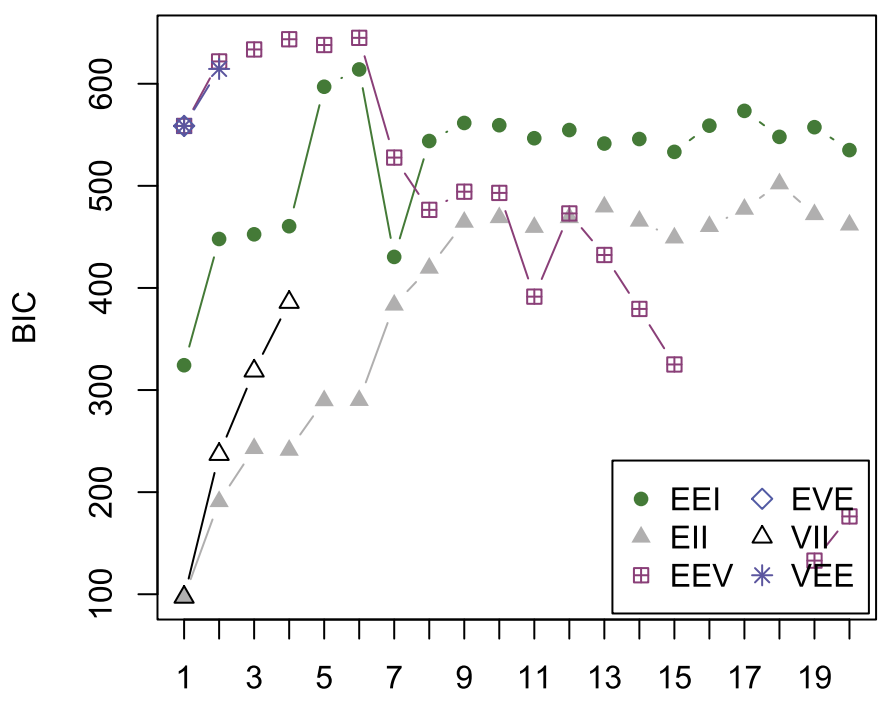

Number of components

(a) BIC performance.

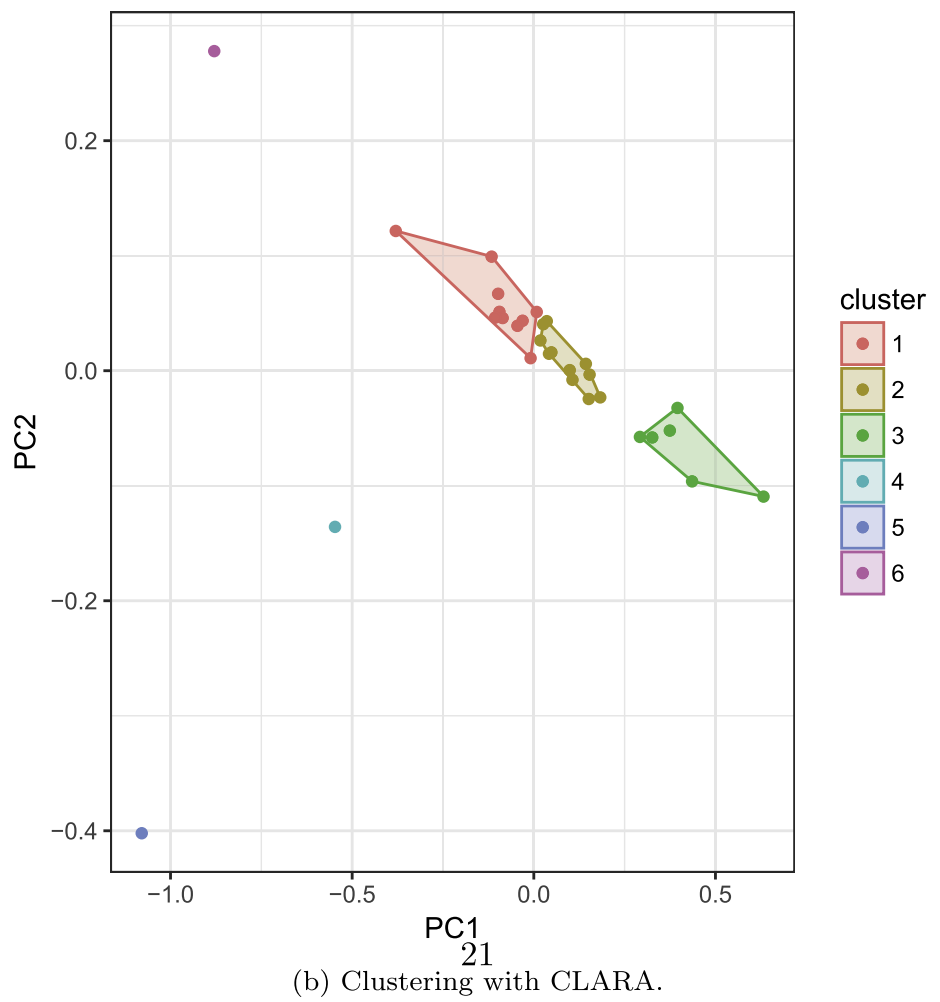

Fig. 7. Clusters for the 1996-2000 FYP.

the $\Delta$ (GDP/Pop) still did not evolve proactively because the GDP variations were not too significant.

\subsubsection{Period of 2011-2015}

The results in this latest period were not in agreement with those of the previous three periods, as most variances could be explained by energy density (see Equation (7)) as before. The second factor responsible is the dematerialization (see Equation (5)). Therefore, the first two eigenvectors were aligned mainly with those features. This is a clear indication that the effects of the priorities become more relevant from the point of view of the data and not from the point of view of the policies themselves.

Considering the BIC criteria (Fig. 10a), the best model-based cluster is the EEIhypothesis, with five clusters. Just as before, outliers were found. These outliers are related to provinces such as XJ in which the reduction of energy usage is very significant in comparison to the variation of the population (in this case, there was a strong reduction in energy figures from the previous FYP). 


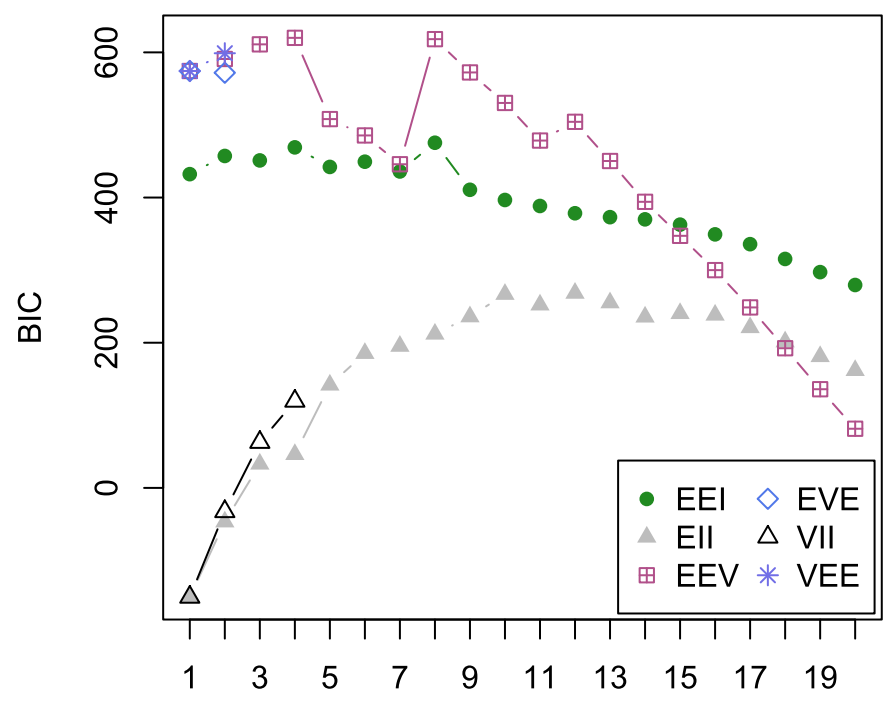

Number of components

(a) BIC performance.

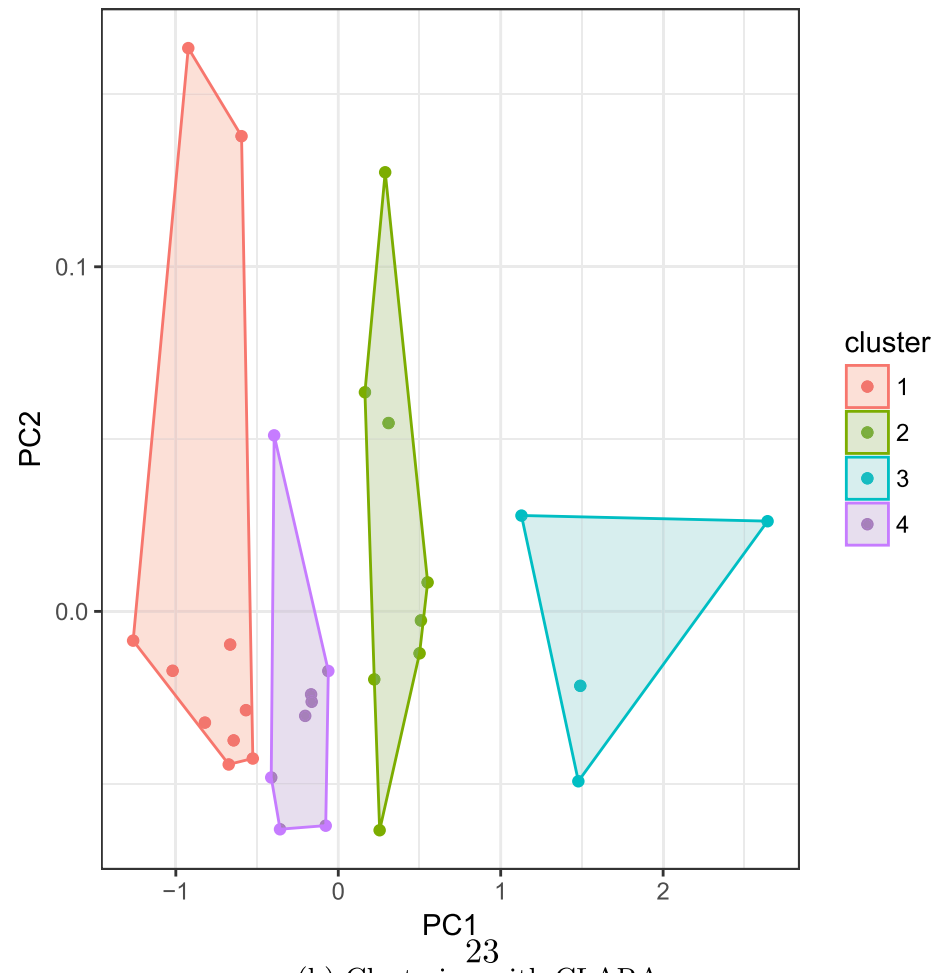

(b) Clustering with CLARA.

Fig. 8. Clusters for the 2001-2005 FYP

The case of JX is completely different, as it exhibits a strong increment in the energy density feature but with an improvement in the amount of $\mathrm{CO}_{2}$ emissions. Therefore, the idea of changes in energy source distribution has become well-supported by the evidence from this data.

In Fig. 10b, the clusters are shown according to the CLARA algorithm proposal.

For most of the large regions, such as $\mathrm{BJ}, \mathrm{TJ}, \mathrm{SH}, \mathrm{HuN}$, and $\mathrm{HuB}$ (cluster 1), an effective reduction in energy density but a poor performance in dematerialization occurred.

The remaining provinces exhibited a more or less homogeneous behavior, showing conservative figures with very small variations in both their energy density and dematerialization features.

\subsection{Driven factors analysis}

Based on the previous analysis, four provinces should be discussed further since several indicators exhibit distinct patterns in 


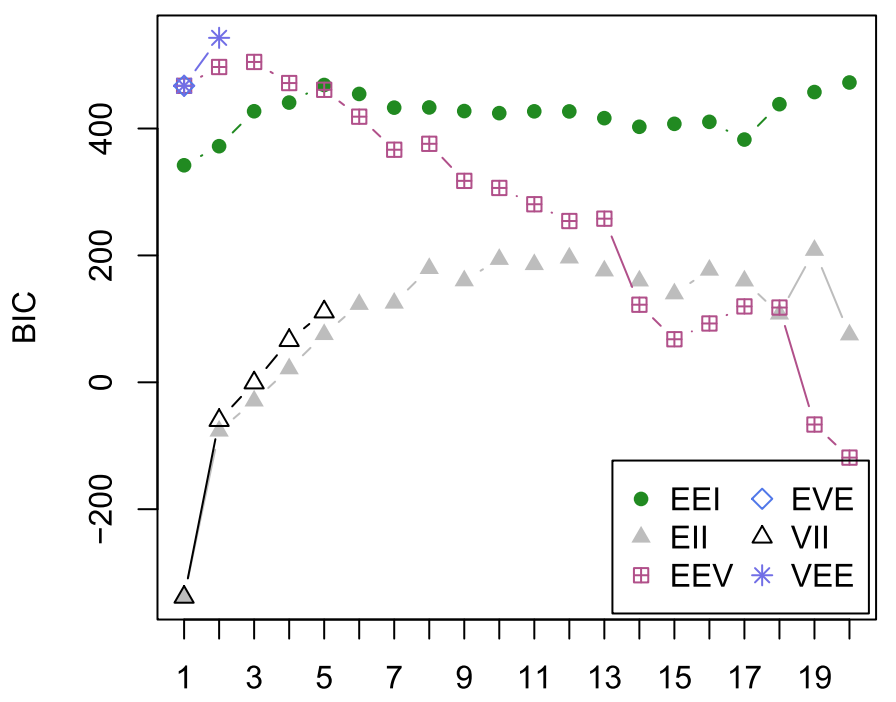

Number of components

(a) BIC performance.

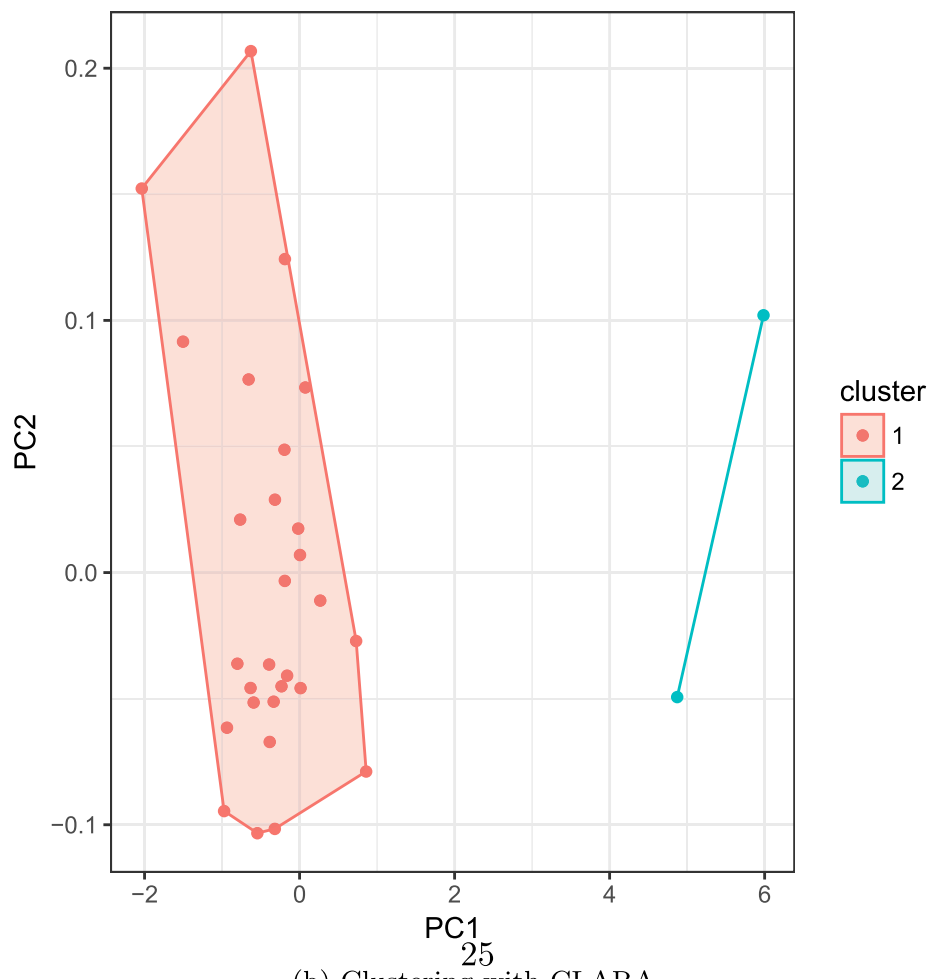

(b) Clustering with CLARA.

Fig. 9. Clusters for the 2006-2010 FYP.

the cluster analysis. These are: CQ JX, XJ and NX.

The case for CQ Province is defined mainly by an incorrect initial estimate of $\mathrm{CO}_{2}$ emissions because of the sources of energy in the ninth FYP. This produced an unbalanced ratio for dematerialization* and decarbonization ${ }^{*}$ (see Fig. 11a and b). For the twelfth FYP, there was only a slight energy reduction with no real changes in the energy sources. It should also be noted that immaterialization* improved in the last FYP because of the strong service transformation of the province's economy. To this end, Tables 6 and 7 illustrate the increasing rates of investment in the coal mining and processing industries and the energy industry. Interestingly, in the period from 1996 to 2005 (9th and 10th FYPs), CQ has increased its investments in the secondary industry and energy sectors.

A similar case can be seen for NX, where servitization is weaker. It can be perceived clearly from the productivity feature, as CQ managed to maintain an increasing ratio but NX did not.

The XJ case is characterized by normal behavior with a significant increment in the energy density over time, which is associated 


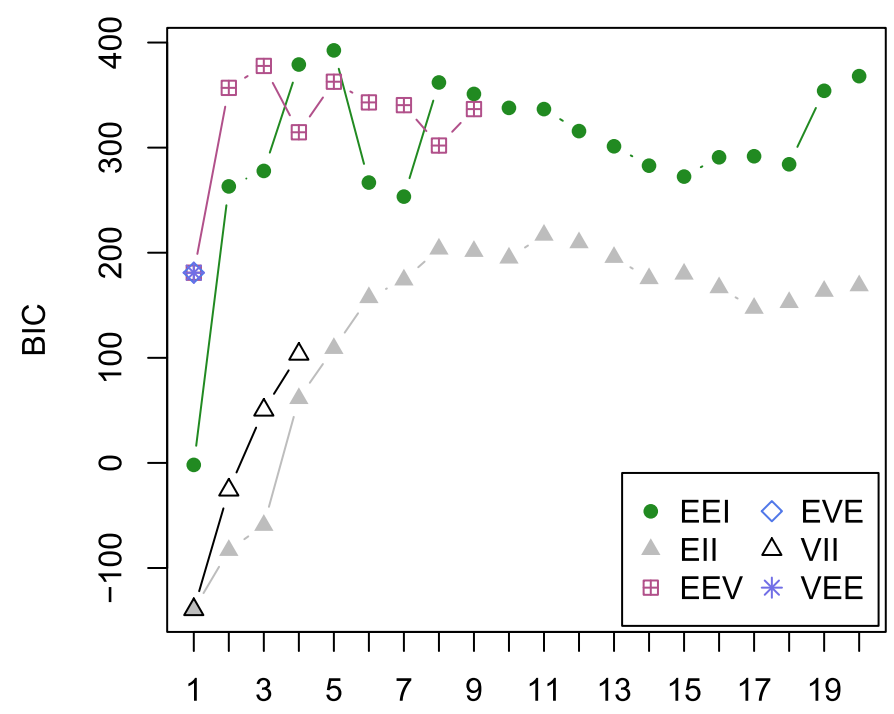

Number of components

(a) BIC performance.

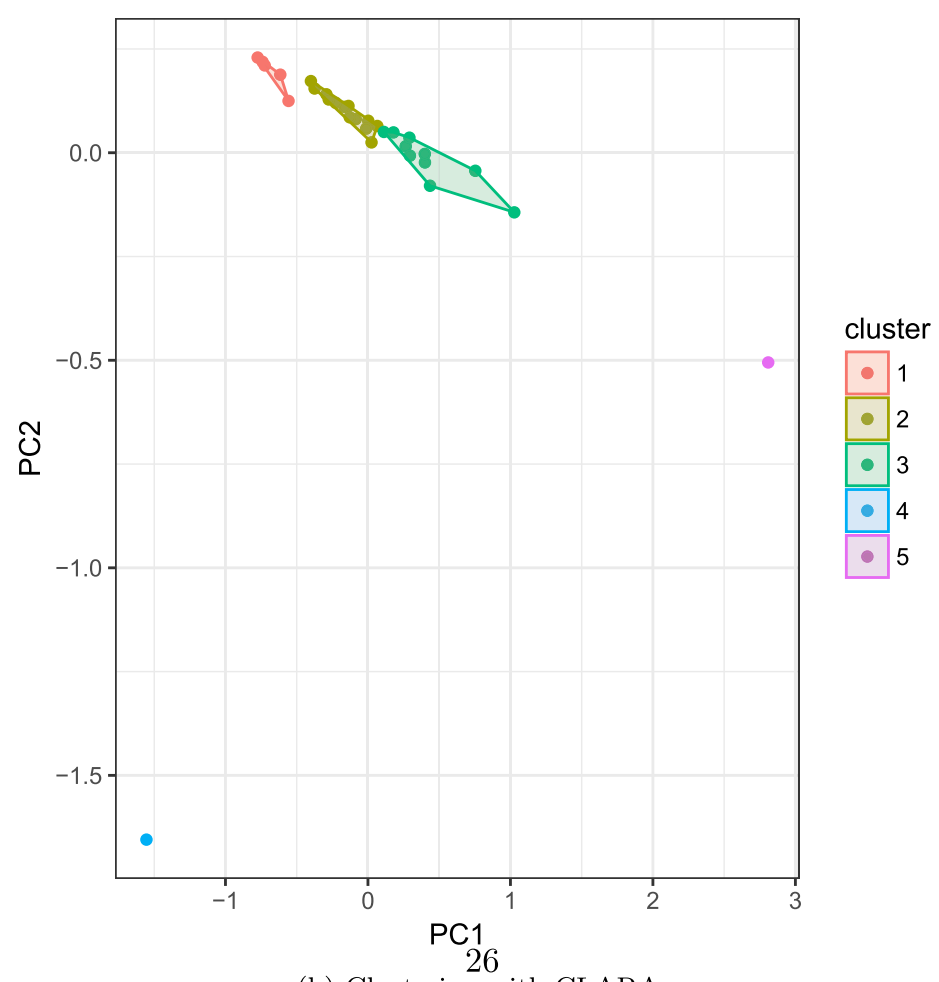

(b) Clustering with CLARA.

Fig. 10. Clusters for the 2011-2015 FYP.

with an economy oriented toward the classical model and structures (see Fig. 12a and b). Therefore, the GDP growth is consistent with this strategy and exhibits an impact on productivity due to the economic crisis.

Finally, the JX case showed a dramatic decrease in dematerialization ${ }^{*}$ and decarbonization. ${ }^{*}$ This is due to a structural change in JX's energy-generating sources, as it implemented a transition to clean energy in the 12th FYP. This province also exhibited a tendency to shift its economic structure to one with more services, making it possible to stabilize the productivity feature. This is consistent with the patterns of change in the percentage of raw coal used out of the total energy for these three provinces.

It is interesting to see how the figures support the contention that productivity is best preserved by shifting the economic orientation to services and by shifting the sources of energy accordingly (see Fig. 13 to immaterialization* for proof of the change to its economic model). 


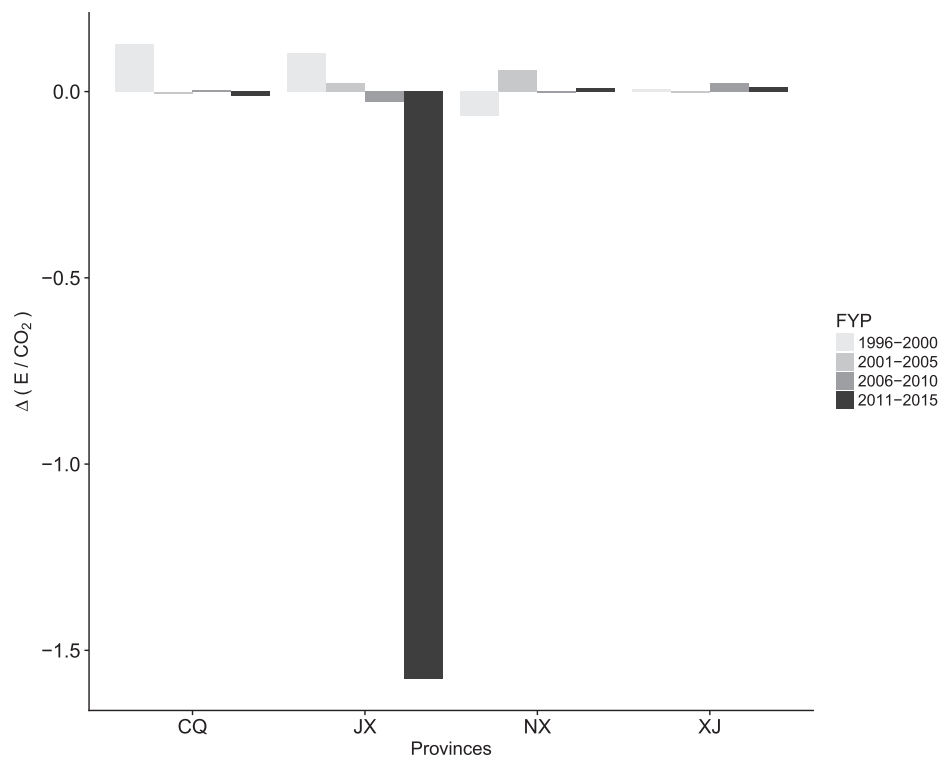

(a) Dematerialization*.

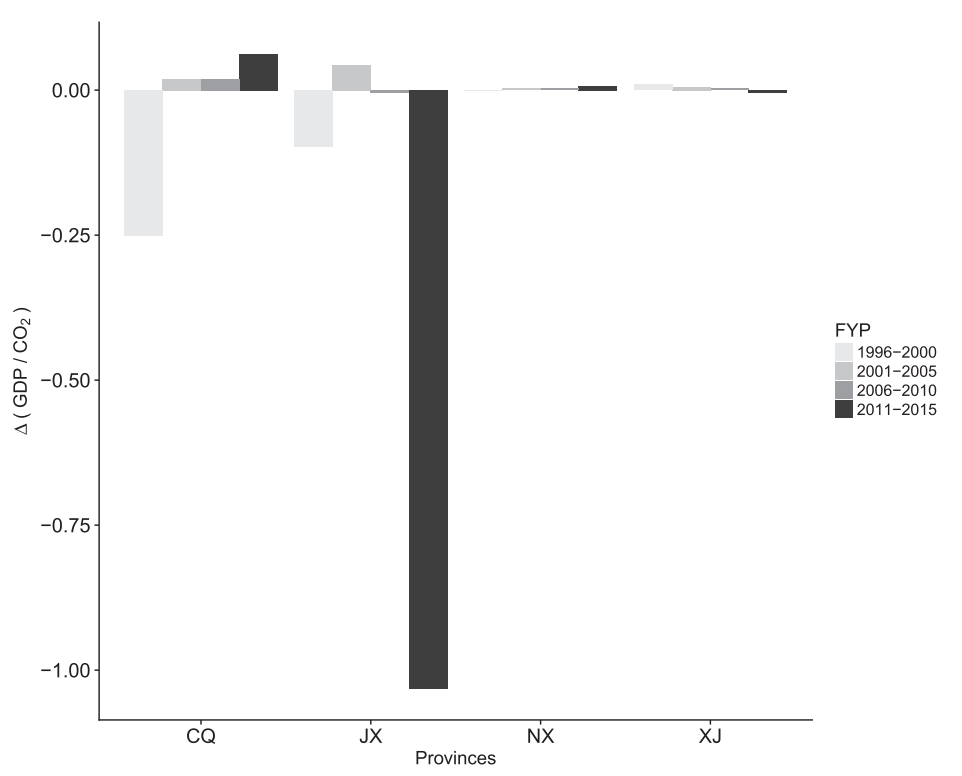

(b) Decarbonization*.

Fig. 11. Comparison of dematerialization and decarbonization behaviors.

From 2006 to 2011, the change in the added value of the tertiary sector was 0.144 in CQ 0.183 in JX, and 0.161 in NX. All of these were above the national average, as seen in Table 5 .

It is also interesting to see the correlation between the effects of the urbanization rate and population on energy usage and emissions, as mentioned in the reference studies [14,26]. The same conclusion cannot be made for China. Fig. 14 shows the urbanization rate increase between 2006 and 2010. It is evident that the increase in the urbanization rates of the GZ, HN, and ShanX provinces are much higher than those in other areas. Therefore, urbanization is probably not the primary factor that distinguishes the patterns among the clusters of the three features.

In a similar way to the work carried out in this paper, a study by Ref. [20] showed that both the change in the power generation from coal to natural gas in the UK and the increase in the industrial and power plant efficiencies of eastern Germany are the main reasons for clustering European countries. The driving factor in that case was the immaterialization feature. The authors also mentioned that the share of different industries was the main reason for the differences among the clusters. However, that study failed to explain the reasons for the cluster differences regarding the decarbonization feature.

\section{Strategic dimensions}

There is a valuable need considering the FYP strategy used in China. This is because the assessment of the plan outcomes at the province level occurred in a more dynamic way than the 
Table 5

Changes in the percentage of added values for the primary, secondary, and tertiary sectors at the provincial level in China.

\begin{tabular}{|c|c|c|c|c|c|c|c|c|c|}
\hline & 1996-2000 & 2001-2005 & $2006-2010$ & 1996-2000 & $2001-2005$ & 2006-2010 & 1996-2000 & $2001-2005$ & 2006-2010 \\
\hline & Primary & & & Secondary & & & Tertiary & & \\
\hline BJ & -0.4687 & -0.4979 & -0.3524 & -0.2287 & -0.1238 & -0.2774 & 0.1976 & 0.0731 & 0.1095 \\
\hline $\mathrm{TJ}$ & -0.3198 & -0.4355 & -0.4722 & -0.0795 & 0.1017 & -0.1760 & 0.1574 & -0.0718 & 0.2530 \\
\hline $\mathrm{HeB}$ & -0.1840 & -0.2304 & -0.1337 & 0.0140 & 0.0901 & -0.0637 & 0.0972 & -0.0170 & 0.1501 \\
\hline ShanX & -0.4506 & -0.3270 & -0.1018 & 0.0141 & 0.1991 & -0.0668 & 0.1639 & -0.1489 & 0.1150 \\
\hline IM & -0.3151 & -0.3868 & -0.3471 & 0.0731 & 0.2555 & 0.0740 & 0.2082 & -0.0411 & 0.0231 \\
\hline LN & -0.2795 & -0.0666 & -0.2441 & -0.0042 & 0.0122 & -0.0136 & 0.1213 & 0.0032 & 0.0768 \\
\hline $\mathrm{JL}$ & -0.3089 & -0.1844 & -0.3000 & 0.0083 & 0.1143 & 0.0801 & 0.2576 & -0.0256 & 0.0287 \\
\hline HLJ & -0.3143 & -0.0602 & 0.0212 & -0.0240 & 0.0357 & -0.2024 & 0.2594 & -0.0314 & 0.3173 \\
\hline $\mathrm{SH}$ & -0.3557 & -0.4073 & -0.3552 & -0.1456 & 0.0192 & -0.2278 & 0.1989 & -0.0052 & 0.2115 \\
\hline JS & -0.2975 & -0.3860 & -0.2224 & 0.0136 & 0.0887 & -0.1957 & 0.1302 & -0.0036 & 0.3471 \\
\hline ZJ & -0.3266 & -0.3846 & -0.2547 & -0.0281 & 0.0455 & -0.1528 & 0.1889 & 0.0342 & 0.2445 \\
\hline $\mathrm{AH}$ & -0.2662 & -0.2941 & -0.2789 & 0.0903 & 0.1476 & 0.1087 & 0.1625 & 0.0313 & -0.0054 \\
\hline $\mathrm{FJ}$ & -0.2610 & -0.2857 & -0.2702 & 0.0715 & 0.1003 & -0.0378 & 0.0726 & 0.0032 & 0.1237 \\
\hline JX & -0.2549 & -0.2988 & -0.3383 & 0.0583 & 0.3892 & -0.0124 & 0.1721 & -0.1752 & 0.1832 \\
\hline SD & -0.2752 & -0.3394 & -0.2055 & 0.0471 & 0.1588 & -0.1832 & 0.1048 & -0.0799 & 0.3817 \\
\hline $\mathrm{HN}$ & -0.1352 & -0.3050 & -0.2552 & -0.0170 & 0.1988 & -0.0675 & 0.1523 & -0.0686 & 0.2534 \\
\hline $\mathrm{HuB}$ & -0.3776 & -0.1607 & -0.2325 & 0.0980 & 0.0888 & -0.0063 & 0.2093 & -0.0178 & 0.0920 \\
\hline HuN & -0.3106 & -0.2321 & -0.2467 & 0.0179 & 0.1243 & 0.0170 & 0.2786 & 0.0101 & 0.0804 \\
\hline GD & -0.3997 & -0.2984 & -0.2242 & -0.0549 & 0.1077 & -0.1243 & 0.2143 & -0.0538 & 0.1742 \\
\hline GX & -0.1974 & -0.1397 & -0.2844 & -0.0220 & 0.1699 & 0.0903 & 0.2058 & -0.0542 & 0.0675 \\
\hline HaiN & -0.0620 & -0.1066 & -0.2804 & 0.1046 & 0.2532 & -0.1825 & 0.0013 & -0.0520 & 0.3391 \\
\hline CQ & -0.3178 & -0.3371 & -0.3088 & -0.0156 & 0.1247 & -0.0635 & 0.2186 & -0.0067 & 0.1444 \\
\hline SC & -0.1473 & -0.1970 & -0.3104 & -0.0905 & 0.1865 & 0.0774 & 0.2306 & -0.0573 & 0.0611 \\
\hline GZ & -0.3120 & -0.3254 & -0.3397 & 0.0845 & 0.0814 & -0.2121 & 0.2710 & 0.1270 & 0.3386 \\
\hline YN & -0.1250 & -0.1261 & -0.2402 & -0.0791 & 0.0536 & -0.1355 & 0.2007 & 0.0115 & 0.2601 \\
\hline ShannX & -0.3555 & -0.3511 & -0.4103 & 0.3187 & 0.1991 & 0.0507 & 0.2291 & 0.0978 & 0.1053 \\
\hline GS & -0.3638 & -0.2205 & -0.1460 & 0.0334 & 0.1828 & -0.0410 & 0.1641 & -0.1181 & 0.0949 \\
\hline $\mathrm{QH}$ & -0.2903 & -0.2063 & -0.2017 & -0.0573 & 0.1254 & -0.1046 & 0.3259 & -0.0316 & 0.1960 \\
\hline NX & -0.3206 & -0.3012 & -0.2149 & 0.0966 & 0.2280 & 0.0048 & 0.0840 & -0.1154 & 0.0519 \\
\hline $\mathrm{XJ}$ & -0.3099 & -0.2556 & -0.2841 & 0.0145 & 0.2027 & -0.0711 & 0.1548 & -0.0978 & 0.1614 \\
\hline Average & -0.2905 & -0.2661 & -0.2578 & 0.0134 & 0.1357 & -0.0726 & 0.1793 & -0.0341 & 0.1693 \\
\hline
\end{tabular}

Table 6

Increase in the rates of investment in the coal mining and processing industries.

\begin{tabular}{|c|c|c|c|c|c|}
\hline \multicolumn{2}{|l|}{$1996-2000$} & \multicolumn{2}{|c|}{$2001-2005$} & \multicolumn{2}{|c|}{$2006-2010$} \\
\hline IM & -87.925 & SD & 69.430 & $\mathrm{AH}$ & -7.478 \\
\hline BJ & -64.516 & $\mathrm{BJ}$ & 95.455 & SD & 6.037 \\
\hline SC & -62.952 & JS & 104.228 & JS & 25.203 \\
\hline NX & -56.425 & $\mathrm{LN}$ & 177.204 & $\mathrm{HN}$ & 116.265 \\
\hline GX & -53.846 & GS & 188.037 & $\mathrm{HeB}$ & 136.008 \\
\hline $\mathrm{HuB}$ & -53.448 & $\mathrm{HeB}$ & 252.495 & $\mathrm{JL}$ & 154.580 \\
\hline $\mathrm{JL}$ & -52.333 & $\mathrm{JX}$ & 300.752 & IM & 158.649 \\
\hline ShannX & -51.546 & HLJ & 326.774 & $\mathrm{CQ}$ & 166.716 \\
\hline $\mathrm{XJ}$ & -44.94 & $\mathrm{FJ}$ & 384.404 & LN & 183.326 \\
\hline YN & -44.293 & ShanX & 556.238 & $\mathrm{QH}$ & 191.886 \\
\hline $\mathrm{AH}$ & -34.986 & $\mathrm{AH}$ & 580.496 & NX & 209.615 \\
\hline HuN & -34.91 & $\mathrm{HN}$ & 778.819 & HLJ & 261.389 \\
\hline GZ & -33.534 & GX & 818.75 & ShanX & 305.824 \\
\hline $\mathrm{HN}$ & -32.787 & $\mathrm{GZ}$ & 906.787 & YN & 325.31 \\
\hline FJ & -26.351 & HuN & 939.091 & $\mathrm{XJ}$ & 328.259 \\
\hline HLJ & -22.113 & ShannX & 1038.771 & GX & 387.302 \\
\hline $\mathrm{QH}$ & -16.667 & YN & 1120 & SC & 437.95 \\
\hline JS & -11.688 & $\mathrm{HuB}$ & 1270.370 & $\mathrm{BJ}$ & 527.907 \\
\hline $\mathrm{HeB}$ & -5.088 & NX & 1553.419 & GS & 626.087 \\
\hline ShanX & -1.606 & $\mathrm{XJ}$ & 1608.108 & $\mathrm{JX}$ & 636.9611 \\
\hline LN & 30.378 & SC & 1807.724 & HuN & 696.807 \\
\hline GS & 31.450 & JL & 2320.280 & $\mathrm{GZ}$ & 708.921 \\
\hline JX & 43.011 & $\mathrm{QH}$ & 2365 & FJ & 726.894 \\
\hline SD & 106.295 & $\mathrm{CQ}$ & 2616.129 & ShannX & 952.751 \\
\hline CQ & 12400 & IM & 5242.488 & $\mathrm{HuB}$ & 1132.432 \\
\hline
\end{tabular}

aggregated individual figures that were used. This is relevant because the goals for the plans are adopted at the national level; however, the implementations are carried out at the province level and different actions are then adopted. Obviously, they will produce different outcomes. Waiting until the end of an FYP to check the level of success is of added, but sometimes limited, value.

The method proposed in this paper for analyzing the outcomes of the FYP implementation at the province level on a yearly basis can be a valuable assessment tool that helps both local authorities and national ones. 
Table 7

Increase in the rates of investment in the energy industry for different time periods and provinces.

\begin{tabular}{|c|c|c|c|c|c|}
\hline \multicolumn{2}{|c|}{ 1996-2000 } & \multicolumn{2}{|c|}{$2001-2005$} & \multicolumn{2}{|c|}{ 2006-2010 } \\
\hline HaiN & -54.481 & $\mathrm{SH}$ & 19.068 & $\mathrm{SH}$ & 5.914 \\
\hline BJ & -27.461 & HuB & 49.099 & ZJ & 19.355 \\
\hline $\mathrm{AH}$ & -24.6811 & HLJ & 105.745 & $\mathrm{AH}$ & 30.044 \\
\hline IM & -10.174 & SD & 108.887 & GD & 30.127 \\
\hline SC & -5.2127 & $\mathrm{LN}$ & 119.521 & JS & 35.082 \\
\hline GD & 21.453 & ZJ & 126.248 & $\mathrm{BJ}$ & 35.652 \\
\hline LN & 23.384 & JX & 133.9269 & CQ & 40.918 \\
\hline HLJ & 28.221 & $\mathrm{XJ}$ & 144.531 & $\mathrm{HuB}$ & 42.05 \\
\hline $\mathrm{SH}$ & 41.005 & BJ & 146.293 & $\mathrm{HN}$ & 44.042 \\
\hline HN & 41.426 & $\mathrm{FJ}$ & 163.644 & GX & 47.934 \\
\hline $\mathrm{TJ}$ & 59.72 & $\mathrm{QH}$ & 166.153 & SD & 48.09 \\
\hline HuN & 65.6740 & $\mathrm{JS}$ & 173.111 & $\mathrm{TJ}$ & 59.737 \\
\hline YN & 66.496 & $\mathrm{HuN}$ & 176.839 & HaiN & 74.08 \\
\hline $\mathrm{HuB}$ & 67.832 & GS & 179.261 & YN & 81.67 \\
\hline $\mathrm{HeB}$ & 73.223 & $\mathrm{TJ}$ & 184.892 & $\mathrm{HeB}$ & 87.843 \\
\hline $\mathrm{FJ}$ & 78.513 & $\mathrm{HN}$ & 205.238 & $\mathrm{QH}$ & 97.431 \\
\hline $\mathrm{JS}$ & 79.683 & $\mathrm{GZ}$ & 205.672 & $\mathrm{IM}$ & 102.229 \\
\hline GS & 81.901 & $\mathrm{JL}$ & 221.288 & $\mathrm{FJ}$ & 110.417 \\
\hline $\mathrm{JL}$ & 82.369 & GD & 223.309 & $\mathrm{JX}$ & 113.125 \\
\hline $\mathrm{XJ}$ & 87.062 & $\mathrm{HeB}$ & 269.718 & HuN & 121.929 \\
\hline SD & 87.322 & ShannX & 306.044 & HLJ & 127.867 \\
\hline $\mathrm{QH}$ & 98.339 & ShanX & 327.785 & SC & 129.414 \\
\hline $\mathrm{NX}$ & 106.814 & GX & 376.265 & $\mathrm{LN}$ & 130.109 \\
\hline ShanX & 119.162 & NX & 389.951 & $\mathrm{JL}$ & 136.647 \\
\hline GX & 123.094 & SC & 392.32 & $\mathrm{GZ}$ & 140.646 \\
\hline ShannX & 126.029 & HaiN & 435.235 & $\mathrm{XJ}$ & 151.704 \\
\hline $\mathrm{JX}$ & 162.268 & CQ & 477.667 & ShanX & 181.361 \\
\hline ZJ & 180.682 & $\mathrm{AH}$ & 532.666 & ShannX & 221.865 \\
\hline GZ & 252.374 & YN & 737.447 & $\mathrm{NX}$ & 248.083 \\
\hline $\mathrm{CQ}$ & 320 & IM & 1321.096 & GS & 268.534 \\
\hline
\end{tabular}

- To the national authorities, the proposed method includes five different dimensions involving figures related to energy intensity, $\mathrm{CO}_{2}$ emissions, economic growth and population in relative values, which provide an excellent way of comparing the aggregated behavior of different provinces. The benefit of producing such a cluster aggregation is that it enables the interpretation of the main drivers responsible for the agglomeration. Indeed, evolution through different years will allow for a closer analysis of the environmental and economic impacts per cluster. This analysis promotes a deeper understanding of the effects, in combination with the agreed upon goals.

- For local authorities, to consider and interpret the information provided by such a dynamic analysis (see the subsections related to the different FYPs in Section 4 improves the efficiency in understanding the individual actions in specific provinces. Such feedback can be dynamically used to reinforce local effects and achieve targets effectively. For instance, knowing the specific effects of one measure over those five factors will be extremely useful in considering which of them to promote. Moreover, particular side effects can be foreseen and alternative actions can be appointed.

The current implementation based on limiting emissions is not only going to produce energy shortages in mainland China; it will also damage the global economy because of the continuously increasing prices for semi-elaborated raw materials, such as fused magnesia, coming mainly from China.

In the real-world context, the decision-making process for the implementation of actions addressing national goals becomes a hard task because of the complexity of the system. Therefore, having detailed information about the effects of actions is always beneficial. To this end, in this work, the analysis that was carried out aligned with the frontier of the FYPs. The main reason is because the assessment was done at the national level in Section 4.
Although the method will not directly change immaterializations or any other relevant variable, it provides insights for better understanding the effects of actions. According to the defined variables, dimensions related to environmental, social, and economical behaviors were involved in the analysis, which included a comprehensive perspective of the effects.

\section{Conclusions}

In this study, the provinces in China were clustered by different features, including relevant ratios such as immaterialization*, dematerialization ${ }^{*}$, decarbonization ${ }^{*}$, energy density, and productivity, in order to understand the similarities within the same cluster and the different patterns among the clusters. For each of the FYPs, an extensive analysis of the clusters was conducted, and their possible driving factors were analyzed and discussed. Some of these findings are summarized below:

- The Chinese provinces that are characterized by specific features were analyzed according to the FYP by using PCA projections. The structures of the explained variances enabled us to identify the relevant features. As they come from the data, these features indicate the effectiveness of the enforced policies.

- GMM models enabled us to identify the most suitable structures of the clusters. Therefore, data-related clustering algorithms were used to group the provinces that have similar aspects.

- The results showed that the CLARA algorithm can successfully separate provinces with large differences (e.g., a cluster with CQJX, XJ, and NX for the immaterialization feature).

- The descriptive analysis suggests that the driving factors for immaterialization could be the changes in the added value of primary, secondary, and tertiary sectors. For dematerialization, the driving factor could be the changes in China's raw coal usage. 


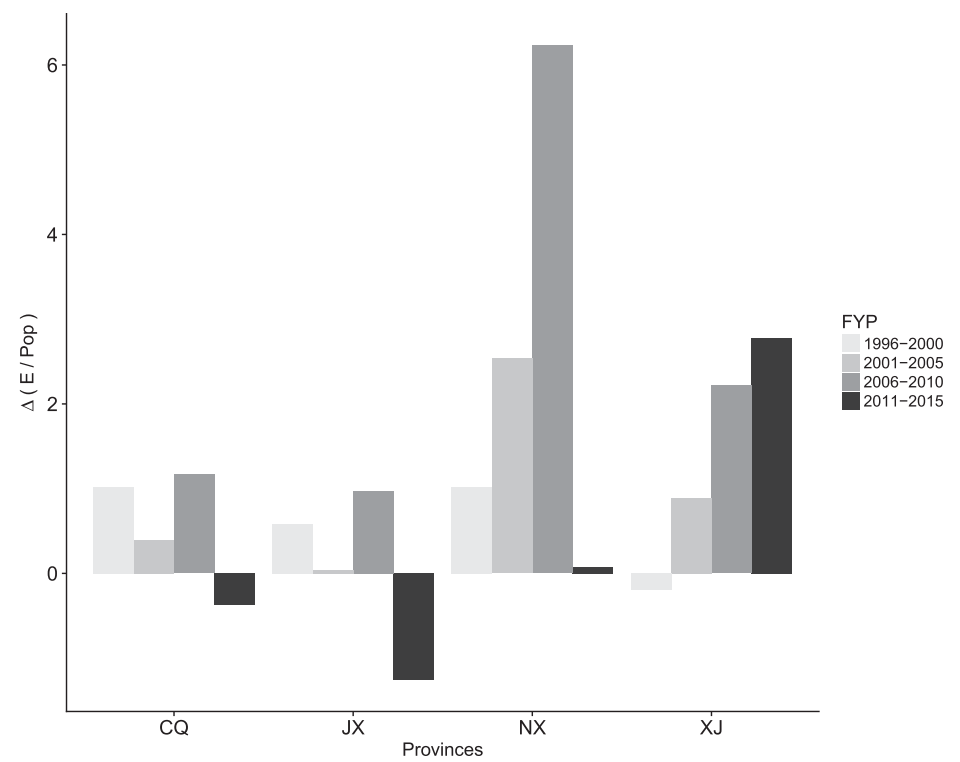

(a) Energy density.

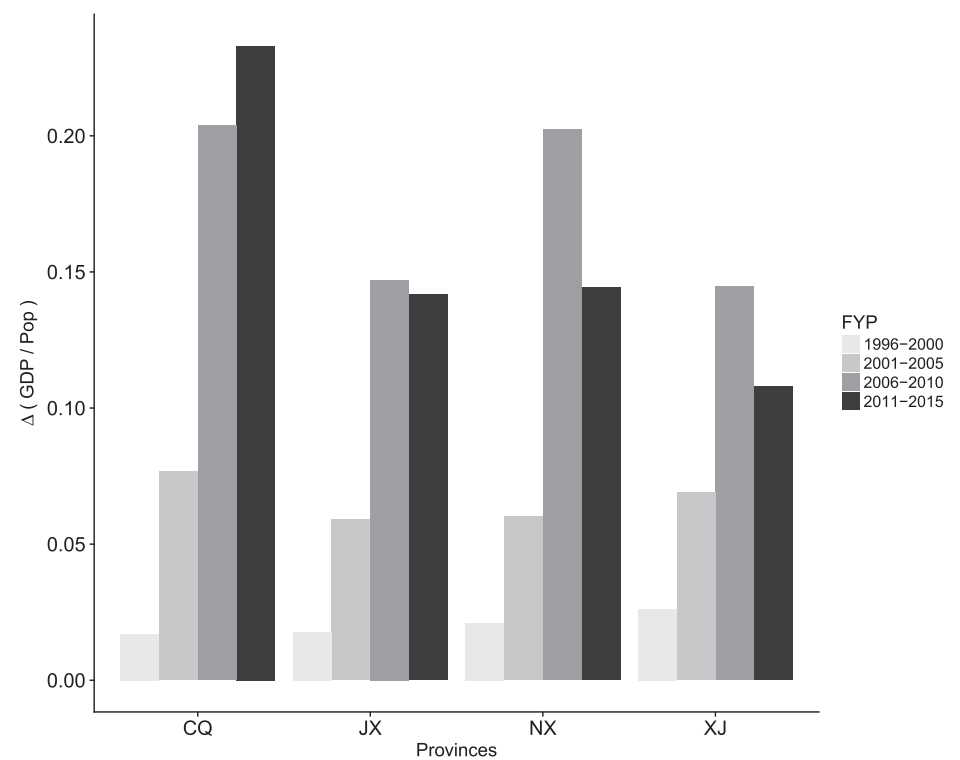

(b) Productivity.

Fig. 12. Comparison of energy density and productivity behaviors.

- Other factors, such as changes in the urbanization rate, are not responsible for the differences in the clusters.

- The existence of clusters themselves makes it clear that enforced policies need to consider the specific situations of each province, making it possible to establish a practicable path for each of them.

- In general terms, the applied methodology enables the development of a comprehensive view of behavior at the provincial level. Therefore, it could be useful for authorities as well as a tool for independent analysis.

Finally, in terms of policies, there is a lack of more integrated instruments for assessing the implemented actions at the national level; this is exactly the value of the proposed method. In addition, it can be used at the local level with a higher frequency to assess the intermediate performance of actions taken, which could be considered a relevant added value.

However, the analysis for identifying driven factors is based on a description analysis. Other factors should also be considered. For instance, the 13 macro influential factors according to the KAYA identity, as shown in Table 8, were taken into account in the studies $[14,25]$. The Kaya identity is a special tool that IPCC provided to reveal the importance of distinct components and factors in the historical data of energy-related $\mathrm{CO}_{2}$ emissions. Integrating the Kaya identity to identify the driving factors for the relevant features using modeling techniques is a future research activity.

Considering additional features related to human welfare, such as the Gini index, may also lead to improvement, although not enough data are presently available at the provincial level. 


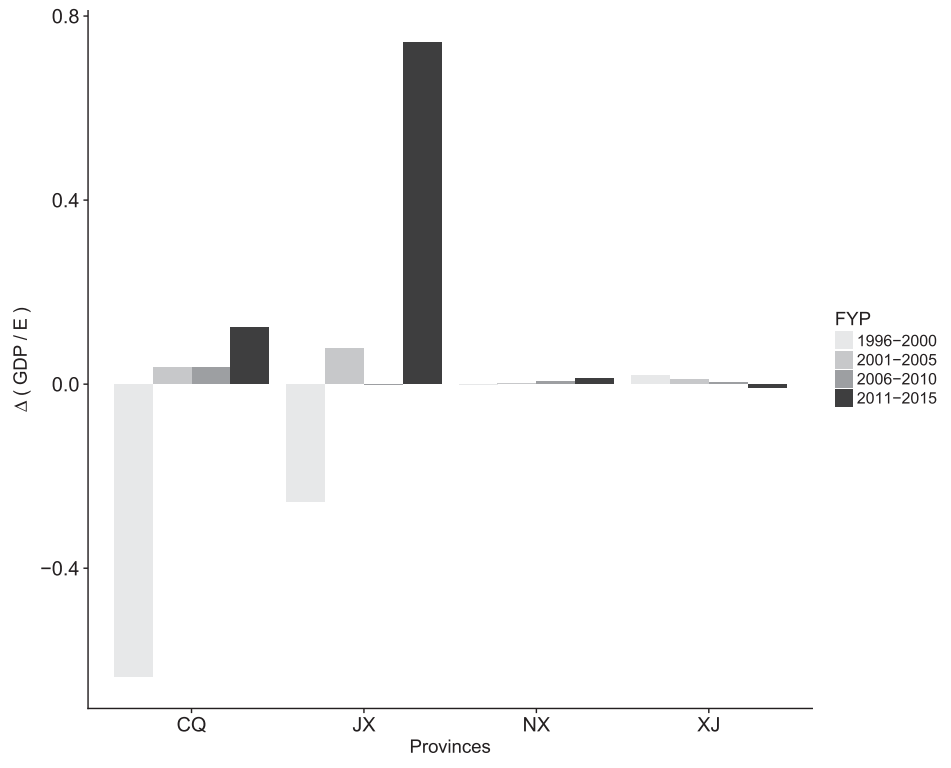

Fig. 13. Comparison of immaterialization ${ }^{*}$ behavior of selected provinces.

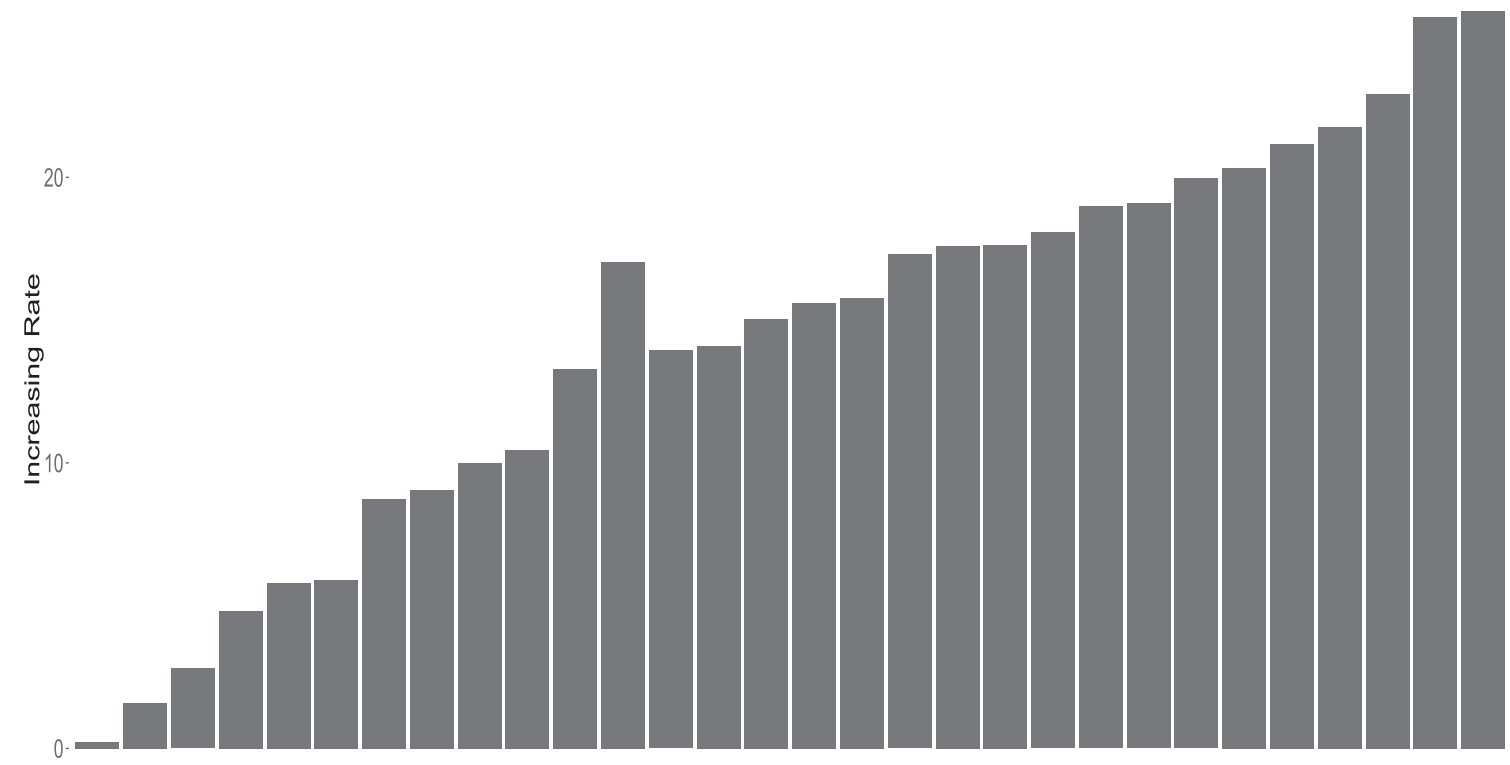

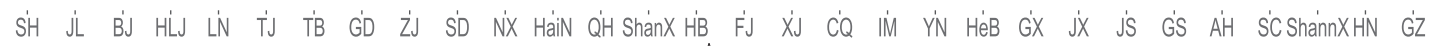

Fig. 14. The urbanization rate of different areas from 2005 to 2013.

\section{Table 8}

Potential influences on $\mathrm{CO}_{2}$ emissions.

\begin{tabular}{l}
\hline Index \\
\hline Population (\%) \\
Per capita added value of the primary industry (\%) \\
Primary industry energy consumption of the per-unit added value (\%) \\
Primary industry carbon emissions of the per-unit energy consumption (\%) \\
Per capita added value of the secondary industry (\%) \\
Secondary industry energy consumption of the per-unit added value (\%) \\
Secondary industry carbon emissions of the per-unit energy consumption (\%) \\
Per capita added value of the tertiary industry (\%) \\
Tertiary industry energy consumption of the per-unit added value (\%) \\
Tertiary industry carbon emissions of the per-unit energy consumption (\%) \\
Per capita income \\
Per capita energy consumption (\%) \\
Per capita carbon emissions from energy consumption (\%) \\
\hline
\end{tabular}

\section{Acknowledgements}

The authors would like to thank the China Scholarship Council (CSC) for the grant that made this research possible.

\section{References}

[1] Al-Mulali U, Ozturk I. The effect of energy consumption, urbanization, trade openness, industrial output, and the political stability on the environmental degradation in the mena (middle east and north african) region. Energy 2015;84:382-9.

[2] Bölük G, Mert M. Fossil \& renewable energy consumption, ghgs (greenhouse gases) and economic growth: evidence from a panel of eu (european union) countries. Energy 2014;74:439-46. 
[3] BP B. BP statistical Review of world energy. 2014 in review. 2015. Online Resource URL:, https://www.bp.com/content/dam/bp/pdf/energy-economics/ statistical-review-2015/bp-statistical-review-of-world-energy-2015-fullreport.pdf. accessed: 2017-11-10.

[4] Cheng Y, Cao K, Woo C, Yatchew A. Residential willingness to pay for deep decarbonization of electricity supply: contingent valuation evidence from Hong Kong. Energy Pol 2017;109:218-27. https://www.sciencedirect.com/ science/article/pii/S0301421517304354. https://doi.org/10.1016/j.enpol.2017. 07.006 .

[5] Chicco G. Overview and performance assessment of the clustering methods for electrical load pattern grouping. Energy 2012;42:68-80. http://www. sciencedirect.com/science/article/pii/S0360544211008565. https://doi.org/10. 1016/j.energy.2011.12.031. 8th World Energy System Conference, WESC 2010.

[6] Ekins P. Economic growth and environmental sustainability: the prospects for green growth. Routledge; 2002.

[7] Guo X, Lu CC, Lee JH, Chiu YH. Applying the dynamic dea model to evaluate the energy efficiency of oecd countries and China. Energy 2017;134:392-9. http://www.sciencedirect.com/science/article/pii/S0360544217310344. https://doi.org/10.1016/j.energy.2017.06.040.

[8] Hotelling H. Analysis of a complex of statistical variables into principal components. J Educ Psychol 1933;24:417.

[9] Jung Y, Park H, Du DZ, Drake B. A decision criterion for the optimal number of clusters in hierarchical clustering. J Global Optim 2003;25:91-111. https:// doi.org/10.1023/A\%3A1021394316112.

[10] Kang D, Lee DH. Energy shocks and detecting influential industries. Energy 2017;125:234-47. http://www.sciencedirect.com/science/article/pii/ S0360544217302694. https://doi.org/10.1016/j.energy.2017.02.087.

[11] Kivyiro P, Arminen H. Carbon dioxide emissions, energy consumption, economic growth, and foreign direct investment: causality analysis for subsaharan africa. Energy 2014;74:595-606.

[12] Korsbakken JI, Peters GP, Andrew RM. Uncertainties around reductions in China's coal use and co $_{2}$ emissions. Nat Clim Change 2016;6:687-90. https:// doi.org/10.1038/nclimate2963. https://www.nature.com/articles/ nclimate2963. accessed: 2017-11-10.

[13] Ma B, Song G, Zhang L, Sonnenfeld DA. Explaining sectoral discrepancies between national and provincial statistics in China. China Econ Rev 2014;30: 353-69.

[14] Mahony TO. Decomposition of Ireland's carbon emissions from 1990 to 2010: an extended Kaya identity. Energy Pol 2013:59:573-81. https://doi.org/ 10.1016/j.enpol.2013.04.0. https://ideas.repec.org/a/eee/enepol/

v59y2013icp573-581.html.

[15] National Bureau SoC. Statistical communiqué of the People's Republic of China on the 2014 national economic and social development. China Statistics Press;
2015. http://www.stats.gov.cn/english/PressRelease/201502/t20150228_ 687439.html.

[16] Ng SW, Mabey N, Gaventa J. Pulling ahead on clean technology: China's 13th five year plan challenges europe's low carbon competitiveness. 2016. Briefing Paper, E3G, London.

[17] Pao HT, Tsai CM. Multivariate granger causality between co2 emissions, energy consumption, fdi (foreign direct investment) and gdp (gross domestic product): evidence from a panel of bric (Brazil, Russian federation, India, and China) countries. Energy 2011;36:685-93.

[18] Salahuddin M, Gow J. Economic growth, energy consumption and co2 emissions in gulf cooperation council countries. Energy 2014;73:44-58.

[19] Su Y, Chen X, Li Y, Liao J, Ye Y, Zhang H, Huang N, Kuang Y. China's 19-year city-level carbon emissions of energy consumptions, driving forces and regionalized mitigation guidelines. Renew Sustain Energy Rev 2014;35: 231-43. http://www.sciencedirect.com/science/article/pii/ S1364032114002421. https://doi.org/10.1016/j.rser.2014.04.015.

[20] Tapio P, Banister D, Luukkanen J, Vehmas J, Willamo R. Energy and transport in comparison: immaterialisation, dematerialisation and decarbonisation in the EU15 between 1970 and 2000. Energy Pol 2007;35:433-51. http://www. sciencedirect.com/science/article/pii/S0301421505003265. https://doi.org/10. 1016/j.enpol.2005.11.031.

[21] Wang W, Li M, Zhang M. Study on the changes of the decoupling indicator between energy-related $\mathrm{CO}_{2}$ emission and gdp in China. Energy 2017a;128: $11-8$.

[22] Wang W, Li M, Zhang M. Study on the changes of the decoupling indicator between energy-related $\mathrm{CO}_{2}$ emission and gdp in China. Energy 2017b;128: 11-8. https://www.sciencedirect.com/science/article/pii/ S0360544217305662. https://doi.org/10.1016/j.energy.2017.04.004.

[23] Wang Y, Chandler W. Understanding energy intensity data in China. Carnegie Endowment for International Peace; 2010. p. 1-35. Type: Policy Outlook.

[24] Yu S, Wei YM, Fan J, Zhang X, Wang K. Exploring the regional characteristics of inter-provincial $\mathrm{CO}_{2}$ emissions in China: an improved fuzzy clustering analysis based on particle swarm optimization. Appl Energy 2012;92:552-62.

[25] Yu S, Wei YM, Wang K. Provincial allocation of carbon emission reduction targets in China: an approach based on improved fuzzy cluster and shapley value decomposition. Energy Pol 2014;66:630-44. http://www.sciencedirect. com/science/article/pii/S0301421513011324. https://doi.org/10.1016/j.enpol. 2013.11.025.

[26] Zhou L, Tian Y, Baidya Roy S, Thorncroft C, Bosart LF, Hu Y. Impacts of wind farms on land surface temperature. Nat Clim Change 2012;2:539. https:/ www.nature.com/articles/nclimate1505/. nclimate1505.

[27] Zhuo L, Cheng B, Zhang J. A comparative study of dimensionality reduction methods for large-scale image retrieval. Neurocomputing 2014;141:202-10. 


\section{Chapter 4}

\section{Discussion}

This research studies the applications of IoT technology in social sectors supported by machine learning techniques. A four-layer application framework was proposed which is composed by data collection layer, data preprocessing and uploading layer, data storage and analysis layer, and practical application layer. A human movement data collection system based on smartwatch, smartphone and NoSQL database was developed. Under the guidance of the proposed framework, the data collection system was applied to a practical case to improve the diagnosis of ET. Forty patients with different tremor levels were involved in the experiments and their movement data were collected both inside and outside hospital during long-term daily lives. A series of machine learning algorithms were adopted to analyze the collected data. The results demonstrated that our system can be used for remotely and continuously monitoring the tremor status of ET patients, recognizing daily activities and help improve the diagnosis of ET. This case study proved the feasibility of the proposed application framework. Similar systems can be developed using the methods presented in this study.

Some results during the research have been published in peer-reviewed journals and conference proceedings. Totally five journal papers and three conference proceedings were published as listed in Table 4.1 .

\subsection{Contributions of each publication}

The main results of this study have been published in five journal articles which are incuded in the previous chapter. The contributions of each article are as follows.

- The publication The development of intelligent healthcare in China reviews the advances of Intelligent healthcare $(\mathrm{IH})$, which is proposed with the fast application of Internet of Things technology in the healthcare area in recent years. It explains how IH can help a developing country as large as China to solve the conflict between limited healthcare resources and the large patient population. Essential policies, milestones, standards, and specifications from the Chinese government since the 1990s were reviewed. Some typical cases and products 
Table 4.1: Publications during the doctoral research

\begin{tabular}{|c|c|c|c|c|}
\hline No. & Title & $\begin{array}{l}\text { Journal/ Con- } \\
\text { ference }\end{array}$ & Year & $\begin{array}{l}5 \text {-Year } \\
\text { IF }\end{array}$ \\
\hline 1 & $\begin{array}{l}\text { Development of a human movement monitoring } \\
\text { system based on wearable devices }\end{array}$ & ESPCO & 2014 & - \\
\hline 2 & $\begin{array}{l}\text { Detection and analysis of tremor using a system } \\
\text { based on smart device and NoSQL database }\end{array}$ & IESM & 2015 & - \\
\hline 3 & $\begin{array}{l}\text { The development of intelligent healthcare in } \\
\text { China }\end{array}$ & $\begin{array}{l}\text { Telemedicine and } \\
\text { e-Health }\end{array}$ & 2015 & 2.418 \\
\hline 4 & $\begin{array}{l}\text { Continuous monitoring of essential tremor using } \\
\text { a portable system based on smartwatch }\end{array}$ & $\begin{array}{l}\text { Frontiers in neu- } \\
\text { rology }\end{array}$ & 2017 & 4.297 \\
\hline 5 & $\begin{array}{l}\text { Comparison of data preprocessing approaches for } \\
\text { applying deep learning to human activity recog- } \\
\text { nition in the context of Industry } 4.0\end{array}$ & Sensors & 2018 & 3.014 \\
\hline 6 & $\begin{array}{l}\text { Activity-aware essential tremor evaluation using } \\
\text { deep learning method based on acceleration data }\end{array}$ & $\begin{array}{l}\text { Parkinsonism \& } \\
\text { related disorders }\end{array}$ & 2018 & 4.855 \\
\hline 7 & $\begin{array}{l}\text { Discovering the patterns of energy consumption, } \\
\text { GDP, and CO2 emissions in China using the } \\
\text { cluster method }\end{array}$ & Energy & 2018 & 5.582 \\
\hline 8 & $\begin{array}{l}\text { Blockchain-based personal health data sharing } \\
\text { system using cloud storage }\end{array}$ & IEEE Healthcom & 2018 & - \\
\hline
\end{tabular}

have been analyzed to present the current situation. It suggests that both government support and the active participation of nongovernmental capital are needed to push forward the whole IH industry.

This review article is a representation of the results obtained during the literature review period at the initial stage of the doctoral study. It demonstrated how IoT technologies can be applied in social sectors such as healthcare and solve challenges in this industry. It made possible the design of the first application framework and helped choosing the application case in heal care domain.

- The publication Continuous monitoring of essential tremor using a portable system based on smartwatch proved the feasibility of using the previously developed smartwatch-based system to continuously monitor tremor levels of ET patients. Tremor identification method according to frequency difference was verified. The tremor was classified into four grades according to the energy calculation results in the frequency domain. Correlation analysis was performed and significant correlation was obtained between neurologist's FTMTRS and patient's FTMTRS mean self-assessment scores; and between patients' self-assessment scores and the classification result based on the collected data. A generalization method was adopted to summarize the detailed classification results and obtain a larger scale analysis result covering a longer period.

The main contribution of this publication is that it proved the feasibility of using the proposed system to evaluate ET and obtained some preliminary analysis results which can help neurologists better understand patient's situation. 
- The publication Comparison of Data Preprocessing Approaches for Applying Deep Learning to Human Activity Recognition analyzed the impact of segmentation methods on deep learning model performance, and compares four data transformation approaches, including raw plot method, which transforms the acceleration data directly to time series images; multichannel method, which treats the data for the three axes as three overlapped color channels; spectrogram method, which represents the frequency features of an inertial signal in the time domain; and spectrogram combined with shallow features method, which combines shallow features with deep-learned features to form the last layer of the deep learning model. The experiment results based on four public datasets demonstrated that the length of data segment significantly impacted the performance of deep learning models; and the multichannel transformation method achieved the best performance in both classification accuracy and training time.

The main contribution of this publication is that it provided a suitable data preprocessing method for deep learning algorithms which was critical for the following research. It also proved that deep CNNs methods can be used for human activity recognition tasks based on acceleration data which paved way for activity-aware tremor evaluation.

- The publication Activity-aware essential tremor evaluation using deep learning method based on acceleration data extended the research presented in the first pubilication and adopted the deep learning methods proposed in the second publication, to create an automated system of a reference scale using motion sensors supported by deep learning algorithms to accurately rate ET severity during voluntary activities. This study obtained a deeper level of tremor evaluation compared with previous studies because it not only focuses on tremor itself, but also takes into account the background activities when tremor occurred. It was realized by using deep learning twice to classify human activities and evaluate tremor respectively. A correlation analysis result shows that the introducing of activity classification can increase the accuracy of tremor severity evaluation against the traditional non-activity-aware models.

The main contribution of this publication is that it applied the most advanced deep learning techniques to the diagnosis of ET based on the acceleration data collected by wearable devices. More accurate evaluation can be obtained by taking into account the background activities compared with other studies. It can help neurologists better understand the tremor variations of ET patients during daily lives and improve treatment plans accordingly.

- The publication Discovering the patterns of energy consumption, GDP, and CO2 emissions in China using the cluster method explores the effects of enforced government policies on environmental matters base on the case of China at provincial level. The relationships among air pollution, energy consumption and economic development are analyzed. This study demonstrated how machine learning techniques can be used to evaluate the effects 
of government policies on real-word issues and how the analysis result can benefit the decision-making process from management point of view. Same approaches can be used to analyze IoT data and provide reliable references for decision makers in different domains such as industry, energy, transportation, healthcare and sports.

In addition to the publications included in the previous chapter, three conference papers related to this research have also been published. The full text is attached in the following chapter.

- The first conference paper Development of a human movement monitoring system based on wearable devices introduced the human movement monitoring system developed at the beginning of the research based on wearable technology. The structure of the system and its main components were explained. The feasibility of using the system to detect typical human daily activities with adequate accuracy was also verified through four practical experiments. This system and its improved version were used during rest of the research as the basis of the practical application.

- The second conference paper Detection and analysis of tremor using a system based on smart device and NoSQL database applied an improved version of the previous human movement monitoring system to monitor the tremor status of ET patients. The tremor identification method based on frequency difference was proposed. This study showed that the proposed system can be used for both tremor detection and human activity recognition through some initiative experiments. The results of this study proved the practicability of using the proposed system to help diagnose ET and encouraged us to reach further in this research line.

- The recent conference paper Blockchain-based Personal Health Data Sharing System Using Cloud Storage proposes a personal health data sharing system using blockchain technology and cloud storage to share the health-related information in a secure and transparent manner. This system could enable users to own, control and share their personal health data securely, in a General Data Protection Regulation (GDPR) compliant way to get benefit from their personal datasets. It also provides an efficient way for researchers and commercial data consumers to collect high quality personal health data for research and commercial purposes. Data sharing based on distributed ledger technologies, such as block chain and Tangle, will be one of the main future research lines.

\subsection{Limitations}

Despite the contributions mentioned previously several limitations exist in this research, mainly due to the limited by time and resources.

- The current activity-aware ET evaluation models can recognize limited number of human activities predefined by neurologists. However, there are large amount of other activities 
during daily lives of ET patients. It is important to recognize as many activities as possible to better evaluate their tremor situation. To realize this target, it is necessary to collect much more human activity data from long-term movement monitoring involving large number of patients.

- One of the main benefits of this research is to help neurologists evaluate and compare the effectiveness of treatment plans. However, this kind of medical experiments requires very long time of observation which is not possible in this research.

- This research only focused on the application of IoT and machine learning in healthcare domain, or more specifically, in the tremor evaluation case, while the proposed application framework can be widely applied in many other sectors like industry area integrated with the Industry 4.0 topic. More study cases could have been involved.

- The most time-consuming part of the research is the movement data collection process. During almost two years of experiments, we have collected a rather large number of movement data from many ET patients. They can be valuable resources for relevant research in this area. These data are difficult to be shared effectively due to the privacy and data security concerns. A more efficient approach to data collection and data sharing should be developed. 


\section{Chapter 5}

\section{Conclusions and future work}

\subsection{Conclusions}

This study explored the application of IoT in different social sectors supported by advanced machine learning techniques. An application framework was designed covering data collection, transferring, storage, analysis and results delivery. Its feasibility was verified through a practical application case. In this case, machine learning techniques such as deep learning were adopted to analyze the data collected from wearable devices in order to improve diagnosis of ET. During this process, the data preprocessing approaches for deep learning were compared in detail from technical point of view. Moreover, the benefit of data mining by means of machine learning for policy makers was discussed through the study of discovering the patterns among energy consumption, air pollution, economic development and government policies. This framework represents the typical IoT application solutions in academic and industrial domains recent years.

One main limitation of the traditional application framework is that no data sharing mechanism is integrated. The collected data are stored in private databases or cloud drives with strict security controls, which hinders the fulfillment of the great potential envisaged by 'Big Data'. In fact, most data that are generated by IoT devices remain locked in what is called 'Data Silos'. Data silos make it impossible or very difficult to share data outside their own closed environments and this leads to enormous quantities of wasted data. According to a report of McKinsey 1 , more than $99 \%$ of data are lost to the void that could potentially contain extremely valuable information if allowed to flow freely in data streams that create an open and decentralized platform that is accessible to any compensating party.

With the increasing concern about data privacy and security issue from public and private users, data protection regulations will become stricter. For example, the European Union has published the General Data Protection Regulation (GDPR) 2 to protect individual data. Such regulations make data sharing even more difficult.

\footnotetext{
${ }^{1}$ https://www.mckinsey.com/mgi/overview/in-the-news/by-2025-internet-of-things-applications-could-have11-trillion-impact

${ }^{2}$ https://eugdpr.org/
} 
Besides the complex data protection regulations, another main obstacle to freely flowing of big data is that, while data sharing is becoming cheaper from a technological perspective, it is prohibitively expensive to transfer fine, granular data in real-time due to intermediary fees $3^{3}$ Another barrier is the lack of ensured authenticity and audit trails of data. Traditional data transmission protocols and databases are susceptible to various attacks, including "man in the middle" attacks and data tampering! ${ }^{3}$ (DLT) provide a solution to overcome these obstacles. For example, the IOTA Tangle [92, based on Directed Acyclic Graph (DAG) and Masked Authenticated Messaging (MAM), has emerged as a powerful solution to address such real-world challenges. It is entirely decentralized and quantum immune. In the Tangle every transaction or data transmission validates two previous ones, which in turn each referenced two previous ones before them, and so on. By this way, the transaction fees are entirely removed and the real-time, fine-granular payment becomes possible. It could also enable zero-cost data integrity, which resolves the security concerns during data transferring.

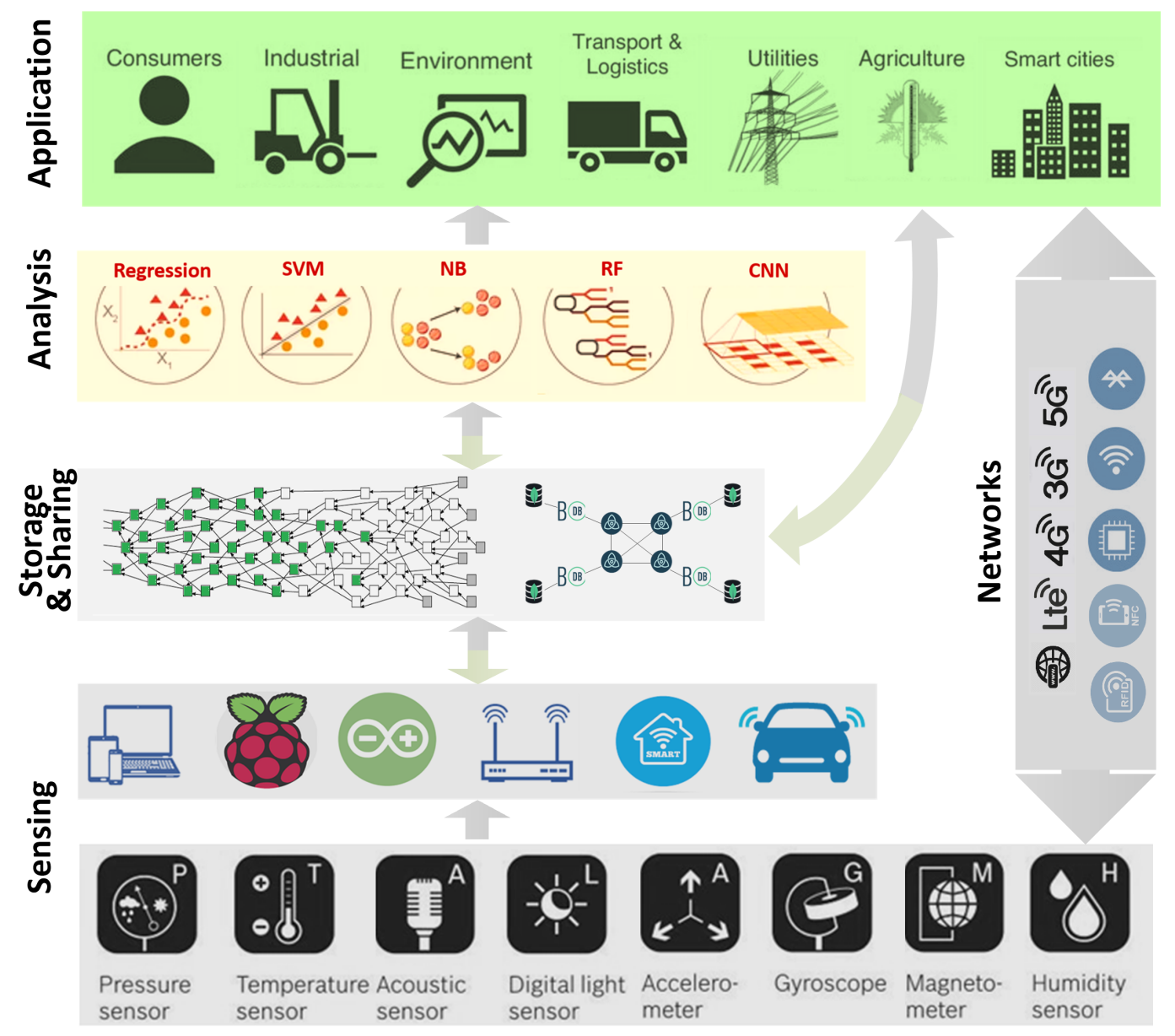

Figure 5.1: IoT application framework supported by machine learning and DLT

Therefore, on the basis of the previously proposed IoT application framework, we propose an advanced architecture by integrating a DLT-supported data sharing layer, as shown in Figure 5.1. Compared with the classical four-layer architecture, in this framework, the data storage

\footnotetext{
${ }^{3}$ https://blog.iota.org/iota-data-marketplace-cb6be463ac7f
} 
and analysis layer on the server side is split into two separate layers, and a data sharing layer is added between them. The data produced by IoT devices are firstly send and saved in private databases or cloud drives. Then there are two options before data analysis: apply machine learning models directly to the data saved in the databases or cloud drives; or share the data in the DLT-based networks, such as the Tangle. In the latter case, the data can be used by multiple parties and the users who shared the data may receive monetary or other type of benefit. This is especially important in the Industrial IoT (IIoT) environment, pushing forward the development of Industrial 4.0.

To summarize, the main contributions of this thesis are:

- Presented an IoT application framework supported by machine learning techniques and proved its feasibility through a practical application case in healthcare.

- Developed a human movement monitoring system using wearable devices and successfully adopted it to improve the diagnosis of ET. This case traversed the complete process of implementing the framework and set examples for other scenarios.

- Compared different data preprocessing approaches and investigated the utilization of deep learning in human activity recognition. It demonstrated the method to select and optimize data analysis algorithms from technical aspect.

- Proposed an advanced activity-aware ET evaluating method based on the acceleration data using deep learning algorithms. It improved accuracy and efficiency of ET diagnosis significantly.

- Explored the benefit of data analysis with machine learning techniques for policy makers and its impact on different social sectors such as economic development, energy consumption and air pollution.

- Designed a pioneering framework for the application of IoT by integrating a data sharing mechanism supported by distributed ledger technologies, which is showing great potential in many domains such as Industrial 4.0.

\subsection{Future work}

Indicated by the limitations of this research mentioned in previous chapters and the advanced application framework proposed in the above section, the following research topics could be addressed in the future.

- To extend the ET experiments to involve more patients during long-term monitoring. The experiments should cover patients' activities both inside and outside hospital. For each patient, the monitoring may last up to several weeks or even months depending on the design of the neurologists. Different treatment plan may be adopted for one patient in 
different periods to compare the effectiveness. The activity recognition algorithms can be improved during this process to include more daily activities.

- To apply the proposed framework to other areas and develop similar data collection and analysis systems. For example, apply it to industry area and integrate it with the Industry 4.0 concept to improve lean production and to reduce safety accidents of workers. In this case, in addition to the wearable devices, the data source may also include environmental sensors, manufacturing equipment and other industrial IoT devices.

- To address the data sharing issue, as mentioned in the new applicaiton framework, we have been using distributed ledger systems, such as blockchain and IOTA Tangle, to develop a costless data sharing platform in the context of IoT. It could provide an opportunity for users to gain the ownership and the privileges of their own data and get benefits from them. Moreover, it could dramatically facilitate the machine-to-machine data transmission and thereby push forward the development of Industrial 4.0. 


\section{Bibliography}

[1] Abadi, M., Barham, P., Chen, J., Chen, Z., Davis, A., Dean, J., Devin, M., Ghemawat, S., Irving, G., Isard, M., et al.: Tensorflow: A system for large-scale machine learning. In: 12th \{USENIX\} Symposium on Operating Systems Design and Implementation (\{OSDI\} 16). pp. 265-283 (2016)

[2] Abraham, A.: Artificial neural networks. handbook of measuring system design (2005)

[3] Ahlrichs, C., Samà, A.: Is frequency distribution enough to detect tremor in pd patients using a wrist worn accelerometer? In: Proceedings of the 8th International Conference on Pervasive Computing Technologies for Healthcare. pp. 65-71. ICST (Institute for Computer Sciences, Social-Informatics and Telecommunications Engineering) (2014)

[4] Al-Fuqaha, A., Guizani, M., Mohammadi, M., Aledhari, M., Ayyash, M.: Internet of things: A survey on enabling technologies, protocols, and applications. IEEE Communications Surveys \& Tutorials 17(4), 2347-2376 (2015)

[5] Alexandra Chang: Hands on with pebble smartwatch, the most successful kickstarter project ever, http://www.wired.com/2012/05/ hands-on-with-pebble-smartwatch-the-most-successful-kickstarter-project-ever/

[6] Amodei, D., Ananthanarayanan, S., Anubhai, R., Bai, J., Battenberg, E., Case, C., Casper, J., Catanzaro, B., Cheng, Q., Chen, G., et al.: Deep speech 2: End-to-end speech recognition in english and mandarin. In: International Conference on Machine Learning. pp. $173-182(2016)$

[7] Ashton, K.: That 'internet of things' thing. RFiD Journal 22, 97-114 (2009)

[8] Atzori, L., Iera, A., Morabito, G.: The internet of things: A survey. Computer Networks 54(15), 2787 - 2805 (Jun 2010), http://wWw.sciencedirect.com/science/article/ $\mathrm{pii/S1389128610001568}$

[9] Atzori, L., Iera, A., Morabito, G., Nitti, M.: The social internet of things (siot)-when social networks meet the internet of things: Concept, architecture and network characterization. Computer networks 56(16), 3594-3608 (2012) 
[10] Ayodele, T.O.: Machine learning overview. In: New advances in machine learning. InTech (2010)

[11] Ayodele, T.O.: Types of machine learning algorithms. In: New advances in machine learning. InTech (2010)

[12] Bahdanau, D., Cho, K., Bengio, Y.: Neural machine translation by jointly learning to align and translate. arXiv preprint arXiv:1409.0473 (2014)

[13] Bain, P.G., Findley, L.J., Thompson, P.D., Gresty, M.A., Rothwell, J.C., Harding, A.E., Marsden, C.: A study of hereditary essential tremor. Brain 117(4), 805-824 (1994)

[14] Bastien, F., Lamblin, P., Pascanu, R., Bergstra, J., Goodfellow, I., Bergeron, A., Bouchard, N., Warde-Farley, D., Bengio, Y.: Theano: new features and speed improvements. arXiv preprint arXiv:1211.5590 (2012)

[15] Bengio, Y., Boulanger-Lewandowski, N., Pascanu, R.: Advances in optimizing recurrent networks. In: 2013 IEEE International Conference on Acoustics, Speech and Signal Processing. pp. 8624-8628. IEEE (2013)

[16] Bengio, Y., Simard, P., Frasconi, P.: Learning long-term dependencies with gradient descent is difficult. IEEE transactions on neural networks 5(2), 157-166 (1994)

[17] Bélissent, J.: Getting clever about smart cities: new opportunities require new business models. November (2010)

[18] Borges, L.M., Rente, A., Velez, F.J., Salvado, L.R., Lebres, A.S., Oliveira, J.M., Araújo, P., Ferro, J.: Overview of progress in smart-clothing project for health monitoring and sport applications. In: Applied Sciences on Biomedical and Communication Technologies, 2008. ISABEL'08. First International Symposium on. pp. 1-6. IEEE (2008)

[19] Breiman, L.: Random forests. Machine learning 45(1), 5-32 (2001)

[20] Brennan, K.C., Jurewicz, E.C., Ford, B., Pullman, S.L., Louis, E.D.: Is essential tremor predominantly a kinetic or a postural tremor? a clinical and electrophysiological study. Movement disorders 17(2), 313-316 (2002)

[21] Chatterjee, A., Aceves, A., Dungca, R., Flores, H., Giddens, K.: Classification of wearable computing: A survey of electronic assistive technology and future design. In: Research in Computational Intelligence and Communication Networks (ICRCICN), 2016 Second International Conference on. pp. 22-27. IEEE (2016)

[22] Chen, T., Li, M., Li, Y., Lin, M., Wang, N., Wang, M., Xiao, T., Xu, B., Zhang, C., Zhang, Z.: Mxnet: A flexible and efficient machine learning library for heterogeneous distributed systems. arXiv preprint arXiv:1512.01274 (2015) 
[23] Cho, K., Van Merriënboer, B., Bahdanau, D., Bengio, Y.: On the properties of neural machine translation: Encoder-decoder approaches. arXiv preprint arXiv:1409.1259 (2014)

[24] Chodorow, K.: MongoDB: The Definitive Guide: Powerful and Scalable Data Storage." O’Reilly Media, Inc." (2013)

[25] Chung, J., Gulcehre, C., Cho, K., Bengio, Y.: Empirical evaluation of gated recurrent neural networks on sequence modeling. arXiv preprint arXiv:1412.3555 (2014)

[26] Collobert, R., Kavukcuoglu, K., Farabet, C., et al.: Torch7: A matlab-like environment for machine learning. In: BigLearn, NIPS workshop. vol. 5, p. 10. Granada (2011)

[27] Collobert, R., Weston, J.: A unified architecture for natural language processing: Deep neural networks with multitask learning. In: Proceedings of the 25 th international conference on Machine learning. pp. 160-167. ACM (2008)

[28] Critchley, M.: Observations on essential (heredofamilial) tremor. Brain 72(2), 113-139 (1949)

[29] Da Xu, L.: Enterprise systems: State-of-the-art and future trends. IEEE Trans. Industrial Informatics 7(4), 630-640 (2011)

[30] Da Xu, L., He, W., Li, S.: Internet of things in industries: A survey. IEEE Transactions on industrial informatics 10(4), 2233-2243 (2014)

[31] David Ludlow: Pebble smart watch review - hands on (Jan 2013), http://www. expertreviews.co.uk/general/1297036/pebble-smart-watch-review-hands-on

[32] Delabrida, S.E., DAngelo, T., Oliveira, R.A.R., Loureiro, A.A.F.: Towards a wearable device for monitoring ecological environments. In: Computing Systems Engineering (SBESC), 2015 Brazilian Symposium on. pp. 148-153. IEEE (2015)

[33] Ding, C., He, X.: K-means clustering via principal component analysis. In: Proceedings of the twenty-first international conference on Machine learning. p. 29. ACM (2004)

[34] Dogu, O., Sevim, S., Camdeviren, H., Sasmaz, T., Bugdayci, R., Aral, M., Kaleagasi, H., Un, S., Louis, E.: Prevalence of essential tremor door-to-door neurologic exams in mersin province, turkey. Neurology 61(12), 1804-1806 (2003)

[35] Elble, R.J.: Essential tremor frequency decreases with time. Neurology 55(10), 1547-1551 (2000)

[36] Eugen Kim: Pebble smartwatch review \& rating (Nov 2013), http://www.pcmag.com/ article2/0,2817,2415719,00.asp

[37] Fahn, S., Tolosa, E., Marín, C.: Clinical rating scale for tremor. Parkinson's disease and movement disorders 2, 271-80 (1993) 
[38] Gama, K., Touseau, L., Donsez, D.: Combining heterogeneous service technologies for building an internet of things middleware. Computer Communications 35(4), 405-417 (2012)

[39] Goodfellow, I., Bengio, Y., Courville, A.: Deep Learning. MIT Press (2016), http://www. deeplearningbook.org

[40] Goodfellow, I.J., Warde-Farley, D., Lamblin, P., Dumoulin, V., Mirza, M., Pascanu, R., Bergstra, J., Bastien, F., Bengio, Y.: Pylearn2: a machine learning research library. arXiv preprint arXiv:1308.4214 (2013)

[41] Graves, A., Mohamed, A.r., Hinton, G.: Speech recognition with deep recurrent neural networks. In: Acoustics, speech and signal processing (icassp), 2013 ieee international conference on. pp. 6645-6649. IEEE (2013)

[42] Gubbi, J., Buyya, R., Marusic, S., Palaniswami, M.: Internet of things (IoT): a vision, architectural elements, and future directions. Future Generation Computer Systems (Feb 2013)

[43] Haerer, A.F., Anderson, D.W., Schoenberg, B.S.: Prevalence of essential tremor: results from the copiah county study. Archives of neurology 39(12), 750-751 (1982)

[44] Hanuska, A., Chandramohan, B., Bellamy, L., Burke, P., Ramanathan, R., Balakrishnan, V.: Smart clothing market analysis (2016)

[45] Hariz, G.M., Blomstedt, P., Koskinen, L.O.: Long-term effect of deep brain stimulation for essential tremor on activities of daily living and health-related quality of life. Acta Neurologica Scandinavica 118(6), 387-394 (2008)

[46] Hartigan, J.A., Wong, M.A.: Algorithm as 136: A k-means clustering algorithm. Journal of the Royal Statistical Society. Series C (Applied Statistics) 28(1), 100-108 (1979)

[47] Heldman, D.A., Jankovic, J., Vaillancourt, D.E., Prodoehl, J., Elble, R.J., Giuffrida, J.P.: Essential tremor quantification during activities of daily living. Parkinsonism \& related disorders 17(7), 537-542 (2011)

[48] Higgins, J.J., Loveless, J.M., Jankovic, J., Patel, P.I.: Evidence that a gene for essential tremor maps to chromosome $2 \mathrm{p}$ in four families. Movement disorders: official journal of the Movement Disorder Society 13(6), 972-977 (1998)

[49] Hinton, G., Deng, L., Yu, D., Dahl, G.E., Mohamed, A.r., Jaitly, N., Senior, A., Vanhoucke, V., Nguyen, P., Sainath, T.N., et al.: Deep neural networks for acoustic modeling in speech recognition: The shared views of four research groups. IEEE Signal processing magazine 29(6), 82-97 (2012) 
[50] Hochreiter, S., Schmidhuber, J.: Long short-term memory. Neural computation 9(8), 1735$1780(1997)$

[51] International Telecommunication Union: ITU internet reports 2005: The internet of things. Tech. rep., Geneva (Nov 2005)

[52] J. Angelo Racoma: Wearable tech: A brief history and a look into the future (Jun 2013), http://www . androidauthority.com/wearable-computing-history-238324/

[53] Jared Newman: Pebble smartwatch pre-orders are sold out, $\$ 10+$ million pledged (May 2012), http://techland.time.com/2012/05/10/ pebble-smartwatch-pre-orders-sold-out/

[54] Jia, Y., Shelhamer, E., Donahue, J., Karayev, S., Long, J., Girshick, R., Guadarrama, S., Darrell, T.: Caffe: Convolutional architecture for fast feature embedding. In: Proceedings of the 22nd ACM international conference on Multimedia. pp. 675-678 (2014)

[55] Jon Collins: The internet of things: a market landscape. Tech. rep. (Jun 2013), http: //research.gigaom.com/report/the-internet-of-things-a-market-landscape/

[56] Joundi, R.A., Brittain, J.S., Jenkinson, N., Green, A.L., Aziz, T.: Rapid tremor frequency assessment with the iphone accelerometer. Parkinsonism \& related disorders 17(4), 288-290 (2011)

[57] Kenney, C., Diamond, A., Mejia, N., Davidson, A., Hunter, C., Jankovic, J.: Distinguishing psychogenic and essential tremor. Journal of the neurological sciences 263(1-2), 94-99 (2007)

[58] Krizhevsky, A., Sutskever, I., Hinton, G.E.: Imagenet classification with deep convolutional neural networks. In: Advances in neural information processing systems. pp. 1097-1105 (2012)

[59] LeCun, Y., Bengio, Y., Hinton, G.: Deep learning. nature 521(7553), 436 (2015)

[60] LeCun, Y., Boser, B., Denker, J.S., Henderson, D., Howard, R.E., Hubbard, W., Jackel, L.D.: Backpropagation applied to handwritten zip code recognition. Neural computation $1(4), 541-551(1989)$

[61] Leo, M., Battisti, F., Carli, M., Neri, A.: A federated architecture approach for internet of things security. In: Euro Med Telco Conference (EMTC), 2014. pp. 1-5. IEEE (2014)

[62] Lin, J., Yu, W., Zhang, N., Yang, X., Zhang, H., Zhao, W.: A survey on internet of things: Architecture, enabling technologies, security and privacy, and applications. IEEE Internet of Things Journal 4(5), 1125-1142 (2017)

[63] Loh, W.Y.: Classification and regression trees. Wiley Interdisciplinary Reviews: Data Mining and Knowledge Discovery 1(1), 14-23 (2011) 
[64] Lorenz, D., Schwieger, D., Moises, H., Deuschl, G.: Quality of life and personality in essential tremor patients. Movement disorders: official journal of the Movement Disorder Society 21(8), 1114-1118 (2006)

[65] Louis, E.: Essential tremor. The Lancet. Neurology 4(2), 100 (2005)

[66] Louis, E.D.: Etiology of essential tremor: should we be searching for environmental causes? Movement disorders 16(5), 822-829 (2001)

[67] Louis, E.D.: Re-thinking the biology of essential tremor: from models to morphology. Parkinsonism \& related disorders 20, S88-S93 (2014)

[68] Louis, E.D., Ferreira, J.J.: How common is the most common adult movement disorder? update on the worldwide prevalence of essential tremor. Movement Disorders 25(5), 534$541(2010)$

[69] Louis, E.D., Fried, L.P., Fitzpatrick, A.L., Longstreth Jr, W.T., Newman, A.B.: Regional and racial differences in the prevalence of physician-diagnosed essential tremor in the united states. Movement disorders: official journal of the Movement Disorder Society 18(9), 1035$1040(2003)$

[70] Louis, E.D., Marder, K., Cote, L., Pullman, S., Ford, B., Wilder, D., Tang, M.X., Lantigua, R., Gurland, B., Mayeux, R.: Differences in the prevalence of essential tremor among elderly african americans, whites, and hispanics in northern manhattan, ny. Archives of neurology 52(12), 1201-1205 (1995)

[71] Louis, E.D., Ottman, R., Allen Hauser, W.: How common is the most common adult movement disorder? estimates of the prevalence of essential tremor throughout the world. Movement disorders: official journal of the Movement Disorder Society 13(1), 5-10 (1998)

[72] Louis, E.D., Wendt, K.J., Albert, S.M., Pullman, S.L., Yu, Q., Andrews, H.: Validity of a performance-based test of function in essential tremor. Archives of neurology 56(7), 841-846 (1999)

[73] Lu, T.C., Fu, C.M., Ma, M.H.M., Fang, C.C., Turner, A.M.: Healthcare applications of smart watches. Applied clinical informatics 7(03), 850-869 (2016)

[74] MacQueen, J., et al.: Some methods for classification and analysis of multivariate observations. In: Proceedings of the fifth Berkeley symposium on mathematical statistics and probability. vol. 1, pp. 281-297. Oakland, CA, USA (1967)

[75] Mahmoud, R., Yousuf, T., Aloul, F., Zualkernan, I.: Internet of things (iot) security: Current status, challenges and prospective measures. In: Internet Technology and Secured Transactions (ICITST), 2015 10th International Conference for. pp. 336-341. IEEE (2015) 
[76] Mamorita, N., Iizuka, T., Takeuchi, A., Shirataka, M., Ikeda, N.: Development of a system for measurement and analysis of tremor using a three-axis accelerometer. Methods of information in medicine 48(06), 589-594 (2009)

[77] Mann, S.: Wearable computing. In: Soegaard, M., Dam, R.F. (eds.) The Encyclopedia of Human-Computer Interaction, 2nd Ed. The Interaction Design Foundation, Aarhus, Denmark (2013), http://www.interaction-design.org/encyclopedia/ wearable_computing.html

[78] Mardonova, M., Choi, Y.: Review of wearable device technology and its applications to the mining industry. Energies 11(3), 547 (2018)

[79] Martens, J., Sutskever, I.: Learning recurrent neural networks with hessian-free optimization. In: Proceedings of the 28th International Conference on Machine Learning (ICML11). pp. 1033-1040. Citeseer (2011)

[80] Matsumoto, Y., Seki, M., Ando, T., Kobayashi, Y., Iijima, H., Nagaoka, M., Fujie, M.G.: Tremor frequency based filter to extract voluntary movement of patients with essential tremor. In: Biomedical Robotics and Biomechatronics (BioRob), 2012 4th IEEE RAS \& EMBS International Conference on. pp. 1415-1422. IEEE (2012)

[81] Mayton, B., Dublon, G., Palacios, S., Paradiso, J.A.: Truss: tracking risk with ubiquitous smart sensing (2012)

[82] McCulloch, W.S., Pitts, W.: A logical calculus of the ideas immanent in nervous activity. The bulletin of mathematical biophysics 5(4), 115-133 (1943)

[83] Michie, D., Spiegelhalter, D.J., Taylor, C.C.: Machine learning, neural and statistical classification (1994)

[84] Miragliotta, G., Perego, A., Tumino, A.: Internet of things : Smart present or smart future? Tech. rep. (Feb 2012)

[85] Mitchell, T.M.: Machine learning. 1997. Burr Ridge, IL: McGraw Hill 45 (1997)

[86] Mostile, G., Giuffrida, J.P., Adam, O.R., Davidson, A., Jankovic, J.: Correlation between kinesia system assessments and clinical tremor scores in patients with essential tremor. Movement Disorders 25(12), 1938-1943 (2010)

[87] Ozcan, K., Velipasalar, S.: Wearable camera-and accelerometer-based fall detection on portable devices. IEEE Embedded Systems Letters 8(1), 6-9 (2016)

[88] Park, S., Jayaraman, S.: Enhancing the quality of life through wearable technology. Engineering in Medicine and Biology Magazine, IEEE 22(3), 41-48 (2003)

[89] Pascanu, R., Mikolov, T., Bengio, Y.: On the difficulty of training recurrent neural networks. In: International Conference on Machine Learning. pp. 1310-1318 (2013) 
[90] Patel, S., Lorincz, K., Hughes, R., Huggins, N., Growdon, J., Standaert, D., Akay, M., Dy, J., Welsh, M., Bonato, P.: Monitoring motor fluctuations in patients with parkinson's disease using wearable sensors. IEEE transactions on information technology in biomedicine 13(6), 864-873 (2009)

[91] Patel, S., Park, H., Bonato, P., Chan, L., Rodgers, M.: A review of wearable sensors and systems with application in rehabilitation. Journal of neuroengineering and rehabilitation $9(1), 21(2012)$

[92] Popov, S.: The tangle (2018)

[93] Pulliam, C., Eichenseer, S., Goetz, C., Waln, O., Hunter, C., Jankovic, J., Vaillancourt, D., Giuffrida, J., Heldman, D.: Continuous in-home monitoring of essential tremor. Parkinsonism \& related disorders 20(1), 37-40 (2014)

[94] Rajput, A., Offord, K., Beard, C., Kurland, L.: Essential tremor in rochester, minnesota: a 45-year study. Journal of Neurology, Neurosurgery \& Psychiatry 47(5), 466-470 (1984)

[95] Rasli, M.K.A.M., Madzhi, N.K., Johari, J.: Smart helmet with sensors for accident prevention. In: Electrical, Electronics and System Engineering (ICEESE), 2013 International Conference on. pp. 21-26. IEEE (2013)

[96] Ravi, D., Wong, C., Lo, B., Yang, G.Z.: A deep learning approach to on-node sensor data analytics for mobile or wearable devices. IEEE journal of biomedical and health informatics 21(1), 56-64 (2017)

[97] Ronao, C.A., Cho, S.B.: Human activity recognition with smartphone sensors using deep learning neural networks. Expert Systems with Applications 59, 235-244 (2016)

[98] Schuhmayer, N., Weber, C., Kieler, M., Voller, B., Pirker, W., Auff, E., Haubenberger, D.: Task-dependent variability of essential tremor. Parkinsonism \& related disorders 41, 79-85 (2017)

[99] Simon, P.: Too Big to Ignore: The Business Case for Big Data. John Wiley \& Sons (2013)

[100] Stephenson, T.A.: An introduction to bayesian network theory and usage. IDIAP-RR 00-03 (2000)

[101] Sundmaeker, H., Guillemin, P., Friess, P., Woelfflé, S.: Vision and challenges for realising the Internet of Things. EUR-OP (2010)

[102] Sutskever, I., Vinyals, O., Le, Q.V.: Sequence to sequence learning with neural networks. In: Advances in neural information processing systems. pp. 3104-3112 (2014)

[103] Tanner, C., Goldman, S., Lyons, K., Aston, D., Tetrud, J., Welsh, M., Langston, J., Koller, W.: Essential tremor in twins an assessment of genetic vs environmental determinants of etiology. Neurology 57(8), 1389-1391 (2001) 
[104] Tehrani, Kiana, Andrew Michael: Wearable technology and wearable devices: Everything you need to know (Mar 2014), http://www.wearabledevices.com/ what-is-a-wearable-device/

[105] Teizer, J.: Wearable, wireless identification sensing platform: self-monitoring alert and reporting technology for hazard avoidance and training (smarthat). Journal of Information Technology in Construction (ITcon) 20(19), 295-312 (2015)

[106] Thanawattano, C., Anan, C., Pongthornseri, R., Dumnin, S., Bhidayasiri, R.: Temporal fluctuation analysis of tremor signal in parkinson's disease and essential tremor subjects. In: Engineering in Medicine and Biology Society (EMBC), 2015 37th Annual International Conference of the IEEE. pp. 6054-6057. IEEE (2015)

[107] Thanvi, B., Lo, N., Robinson, T.: Essential tremor - the most common movement disorder in older people. Age and ageing 35(4), 344-349 (2006)

[108] Wagstaff, K., Cardie, C., Rogers, S., Schrödl, S., et al.: Constrained k-means clustering with background knowledge. In: ICML. vol. 1, pp. 577-584 (2001)

[109] Wile, D.J., Ranawaya, R., Kiss, Z.H.: Smart watch accelerometry for analysis and diagnosis of tremor. Journal of neuroscience methods 230, 1-4 (2014)

[110] Wu, X., Kumar, V., Quinlan, J.R., Ghosh, J., Yang, Q., Motoda, H., McLachlan, G.J., Ng, A., Liu, B., Philip, S.Y., et al.: Top 10 algorithms in data mining. Knowledge and Information Systems 14(1), 1-37 (2008)

[111] Yan, L., Zhang, Y., Yang, L.T., Ning, H.: The Internet of Things: from RFID to the next-generation pervasive networked systems. CRC Press (2008)

[112] Young, T., Hazarika, D., Poria, S., Cambria, E.: Recent trends in deep learning based natural language processing. ieee Computational intelligenCe magazine 13(3), 55-75 (2018)

[113] Zeng, M., Nguyen, L.T., Yu, B., Mengshoel, O.J., Zhu, J., Wu, P., Zhang, J.: Convolutional neural networks for human activity recognition using mobile sensors. In: Mobile Computing, Applications and Services (MobiCASE), 2014 6th International Conference on. pp. 197-205. IEEE (2014)

[114] Zheng, X., Ordieres-Meré, J.: Development of a human movement monitoring system based on wearable devices

[115] Zheng, X., Ordieres-Meré, J.: Detection and analysis of tremor using a system based on smart device and nosql database. In: Industrial Engineering and Systems Management (IESM), 2015 International Conference on. pp. 242-248. IEEE (2015) 
